\title{
Stay in or drop out
}

The pathways to school dropout from a public health perspective

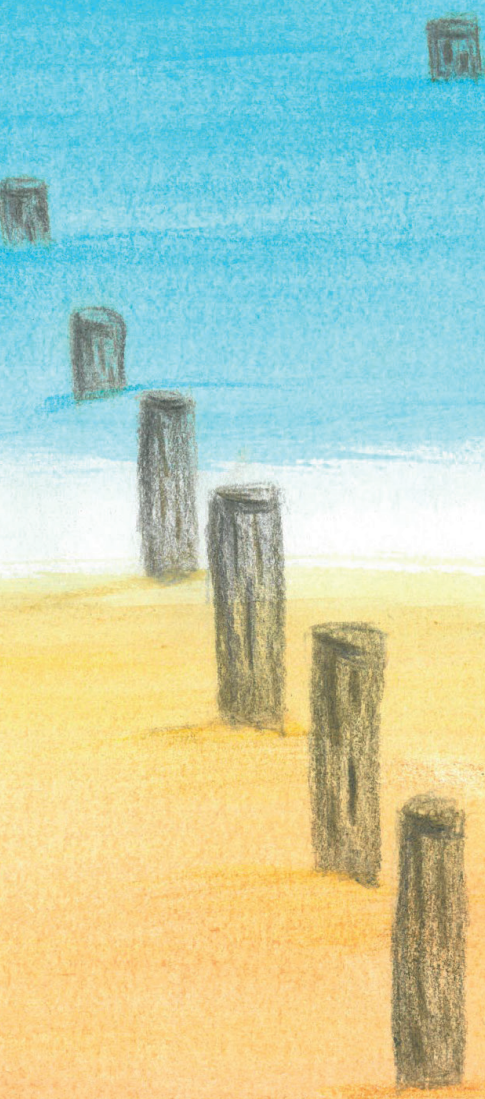

Marie-José Theunissen-Lamers 
Cover design: Karlijn Theunissen

Layout, production: Datawyse | Universitaire Pers Maastricht

ISBN: 9789461595867

(C) Marie-José Theunissen-Lamers, Maastricht 2016

All rights reserved. Brief excerpts from this publication may be used for scientific and educational purpose provided that the source is acknowledged. 


\title{
Stay in or drop out
}

\section{The pathways to school dropout from a public health perspective}

\author{
PROEFSCHRIFT \\ ter verkrijging van de graad van doctor aan de Universiteit Maastricht, \\ op gezag van de Rector Magnificus, Prof. dr. Rianne M. Letschert, \\ volgens het besluit van het College van Decanen, \\ in het openbaar te verdedigen \\ op woensdag 16 november 2016 om 14:00 uur \\ door
}

Marie-José Theunissen-Lamers 


\section{Promotores}

Prof. dr. F.J.M. Feron

Prof. dr. H. Bosma

\section{Copromotor}

Dr. P. Verdonk, VUMC Amsterdam

\section{Beoordelingscommissie}

Prof.dr. T.A.M.J. van Amelsvoort (Voorzitter)

Prof.dr. M.R.J. Crul (Vrije Universiteit Amsterdam)

Prof.dr. K.Horstman

Prof.dr. Ir. M.W.J. Jansen

Prof.dr. S.A. Reijneveld (Universitair Medisch Centrum Groningen)

The research presented in this thesis was conducted at the School for Public health and Primary care: CAPHRI, Department of Social Medicine, of Maastricht University. CAPHRI participates in the Netherlands School of Primary Care Research CaRe.

The research was funded by the Dutch Organisation for Health Research and Development (ZonMw, The Hague, Netherlands), project number 20400.9003 and the Regional Public health Service (GGD) Brabant Zuidoost. 
Aan Hans, Bas, Jasper en Karlijn 



\section{Table of contents}

List of abbreviations

Chapter 1 General introduction

Chapter 2 The early identification of risk factors on the pathway to school dropout in the SIODO study: a sequential mixed-methods study

Chapter 3 Why Wait? Early Determinants of School Dropout in Preventive Pediatric Primary Care

Chapter 4 Are Barbie and Ken too cool for school? A case-control study on the relation between gender and dropout

Chapter 5 Low control beliefs in relation to school dropout and poor health: findings from the SIODO case-control study

Chapter 6 "I think that the whole family needed help, but they only focused on me" An interview study with young adults about their childhood and dropping out of school

Chapter 7 "Don't you have faith in them?" A focus group study with stakeholders in the process toward school dropout

Chapter 8 General discussion

Summary

Samenvatting

Valorisatie

Dankwoord

Curriculum Vitae

Publications

205 



\section{List of abbreviations}

AFIS Adolescent Femininity Ideology Scale

AMIRS Adolescent Masculinity Ideology in Relationships Scale

BPS Biopsychosocial

CED Compulsory Education Department

DSM Diagnostic and Statistical Manual of Mental Disorders

HAVO Higher general secondary education

HBO Higher professional education

$\mathrm{HC} \quad$ Health Care

ICF-CY International Classification of Functioning and Health for Children and Youth

ISR Inauthentic Self in Relationship

MBO Intermediate vocational education

$\mathrm{PCYH} \quad$ Preventive Child and Youth Healthcare

PPPC Preventive Paediatric Primary Care

SES Socioeconomic Status

SIODO Stay In Or Drop Out

VMBO Preparatory vocational secondary education

VWO Pre-university education

WHO World Health Organization

Wo Academic education

YHC Youth Health Care

NB: PCYH, PPPC and YHC are different names of the same preventive youth healthcare (0-18 years). PPPC is more used in USA, while YHC and PCYH are more European terms. Because chapter 3 is published in a US journal, PPPC is used. However, in most chapters of this thesis YHC has been used. 



\section{Chapter 1}

General introduction 



\section{Introduction}

Early school leaving is a concern of most European countries, including the Netherlands. Dropping out of school has major societal consequences. When young people drop out of school, they have an increased risk of dropping out of society to a life of anti-social behaviour and possibly crime $(1,2)$. This will bring higher costs for society, just like less economic growth because of a lower educated population, less tax income and higher costs for social welfare and health care. Preventing school dropout will not only save costs for society, but will also save personal suffering. Youngsters, dropping out of school are often stigmatized and considered to be lazy, oppositional or rabble. However, many problems may be due to adverse circumstances in childhood. If health and developmental problems might be implicated in the pathways leading to school dropout, we must consider reframing early school leaving as a public health issue (3). To avoid further stigmatisation, in this thesis I will examine the role of health problems in a life course perspective from early childhood to adolescence. This will contribute to the future development of more effective, personalized interventions that will help youth health care $(\mathrm{YHC})$ professionals, teachers, and parents to prevent students from dropping out of school. This introduction is outlined as follows. First, I discuss why early school leaving must be seen as a public health issue. Then, I describe gender differences in drop-out rates, the concept of growing into deficit and the aim of this thesis. Subsequently, the Dutch educational system and the Dutch YHC are summarized. Finally, I describe the study design and the research questions to be addressed in this study.

\section{Early school leaving from a public health perspective}

In contrast to the USA, most European countries including the Netherlands use an educational qualification framework and have defined the minimum requirements for an entrance in today's labour market (4). In the Netherlands, this basic qualification is equivalent to a certificate of higher general secondary education (HAVO), pre-university education (VWO) or intermediate vocational education (MBO), Level $2(4,5)$. Youngsters without a basic qualification, who do not attend school anymore are considered early school leavers or school drop outs. Without a basic qualification it is hard to get a well-paid job and many youngsters will end up unemployed, risking social exclusion and being dependent on social welfare (6).

The Dutch government has implemented several laws, policies and interventions to prevent early school-leaving. However, most of these policies and interventions are targeted at factors that operate late in the process towards school dropout (7). Few interventions are aimed at tackling the problem at its (potential) roots by identifying children with physical, mental and social problems early in life. Moreover, dropping out of school is generally considered an educational problem and hardly seen from a (public) health perspective, although education level is closely linked to health status and to health disparities (3). 
People in lower socioeconomic positions, traditionally defined by educational, occupational and income level, tend to die at a younger age and within their shorter lives, they have a higher prevalence of many health problems $(8,9)$. A lower education level is also associated with increased risky health behaviours such as smoking, overweight and lack of physical activity. Moreover, the health gap between high and low educated people has grown in recent decades (10).

By the year 2020 the Dutch government aims to reduce the socioeconomic health inequalities by 25\% compared with 2003 (11). In order to tackle these socioeconomic health inequalities, more insight into the determinants is necessary. The influential Black Report, which showed the unequal distribution of ill-health and death among the population of Britain, suggested two main explanations for socioeconomic health inequalities (12). The causal mechanism implies that socioeconomic status (SES) influences people's health status through differential exposure to health-related behavioural, structural and material, and psychosocial stress-related determinants. There is much evidence for such mechanisms. The selection mechanism implies the opposite; that health status determines socioeconomic position. Socioeconomic health differences have been examined less often from this perspective. The selection mechanism assumes that people with initially poorer health more often move downward, whereas people with initially better health more often move upward in the social hierarchy. Related to this, indirect selection implies that downward and upward social mobility can be caused not only by health itself, but also by health-related risk factors or personality traits which are (partly) formed in youth (13). This thesis aims to gain insight into how the (indirect) selection mechanism contributes to school dropout, for this will offer opportunities to predict school dropout at an early age.

With good education being such a strong predictor of good health (3) and poor health being involved in pathways towards school dropout, early school leaving must be considered a public health problem. Moreover, good education contributes to decreased socioeconomic health differences and thus, cannot be absent on the public health agenda. Below, we first describe social and cultural gender differences in school dropout, which also deserve more attention in public health.

\section{Gender differences in early school leaving}

In contrast to sex, which generally refers to the biological differences between women and men, gender is hardly investigated in medical research (14). Gender refers to the culturally determined beliefs, behaviours and characteristics that are associated with, but not determined by, sex. Gender differences are learned, may change over time and vary significantly both within and between cultures $(15,16)$. However, gender differences are consistent cross-cultural findings in dropout rates (17). Girls graduate faster and with higher grades from higher levels of education than boys, and these gender differences in academic achievements begin to occur during adolescence. Feminization 
of education, with a majority of female teachers and a curriculum which is better suited to the learning process of girls, has been put forward as an important explanation for the higher amount dropouts in boys. However, in the Dutch Emancipation Monitor 2008 , the issue of feminization of education is summarized and the authors state that gender difference do occur not because boys are doing so much worse than before, but because academic achievements in girls have increased (18). In the Netherlands, 6 out of 10 school dropouts are male, and at each higher level fewer girls drop out compared with the number of boys (18). The timing (puberty) suggests on the one hand a hormonal influence, and on the other hand diverging lives of boys and girls (19). Peers play an important role in adolescents' life. Peers' beliefs about school and academic achievement influence their friends' behaviour and attitudes and when youngsters have friends who dropped out of school, they are more likely to drop out of school themselves (20). Furthermore, peer influences differ for boys and girls (19). From birth on children learn how they are expected to behave as a boy or as a girl through reward and punishment and through modelling and imitation (21). Boys drop out of school more often than girls and boys are more likely to engage in deviant behaviours such as the use of violence. Such behaviours may provide means to earn respect, prove masculinity, and meet peer expectations which make them 'too cool for school'. In general, early adolescents are rather friends with good peers than with deviant peers (22). However, lack of prosocial connections can lead them to form connections with deviant peers (23). Children with early behaviour problems are at risk of developing academic problems and experiencing rejection from their prosocial peers. When connecting with deviant peers, they have an increased risk of engaging in other delinquent acts (23).

In this thesis I examine how social and cultural gender differences are involved in the pathways to school dropout.

\section{Growing into deficit}

Dropping out of school is not a discrete event, but a dynamic developmental process that often begins early in life $(24,25)$. Nevertheless, most interventions to prevent early school leaving are targeted at factors that operate late in the dropout process $(7,26)$. This thesis focuses on early symptoms, to find signs of heightened risks of school dropout as early as possible.

Figure 1 shows the principle of growing into deficit (27). In the current health system, therapy is followed after a diagnosis has been given. Mostly, symptoms children show vary along a continuum from normal variations, to problems and eventually disorders (28) and deficiencies become clearer over time ("growing into deficit"). When a child demonstrates symptoms but not to a level severe enough to warrant a diagnosis, the symptoms can still cause significant impairment and distress, affecting the child's development $(28,29)$. The number of children who do not meet the criteria for a diagnosis, but who have clinically significant impairment is estimated to be equal to twice 
the number of children with a diagnosis (30). Children and youth without a diagnosis, rarely receive help and guidance, compared to diagnosed children (31). By focusing on symptoms, rather than on diagnoses, a shift is needed in the current medical model from a conventional approach of collecting information to support an existing diagnose ("find it and fix it") to a more dynamic approach of identifying health risks and prevent negative outcomes ("predict it and personalize it"). Moreover a more personalized approach is needed to detect biological vulnerabilities ("baseline risk"), assess the effects with environmental factors and anticipate on individual needs (32). The YHC, which will be discussed below, has a unique position in the health care system and may play an important role in moving towards predicting health and developmental risks.

The 'growing into deficit' concept also applies to the complex and gradual process of school dropout. Despite underlying vulnerabilities and negative environmental influences children may be able to survive and function adequately at primary school, where learning is externally structured and most children experience social support from their teacher. In secondary and tertiary school, these children face increased expectations for learning performance, autonomy and social skills even though their deficiencies have not changed. In these instances, the decline in functioning actually reflects the fact that the child has reached an age at which new academic and social demands meet the limits of their always existing possibilities and limitations.

Detecting, monitoring and attending (clusters of) symptoms in early life, before they cluster to a diagnosis, may contribute to a further reduction of dropout rates. Moreover, by identifying children at risk in an early stage, the YHC can intervene before further harm has occurred.

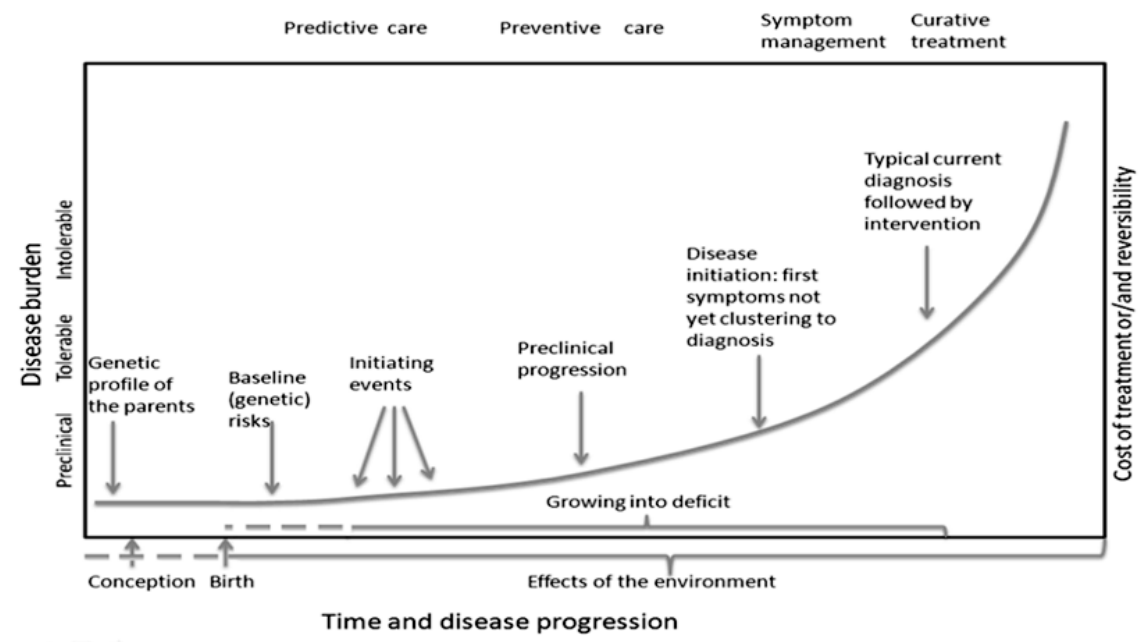

Figure 1. Growing into deficit. Ref: Syurina EV, Hens K, Feron FJM. Literature Review: Use of Family History for Primary Paediatric Care as the Next Step Towards use of Genomics in Healthcare. Current Pediatric Reviews 2013;9(4): 353-372. 


\section{Aim of this thesis}

The aim of this thesis is to provide insight into the life-course pathways leading towards school dropout and into the socioeconomic and gender differences found in these pathways. Understanding how the health-related determinants in the different time periods in children's and youth's lives explain school dropout in young adulthood (18-23 years), will contribute to the future development of more effective, personalized interventions in $\mathrm{YHC}$ aimed at reducing school dropout at an as early as possible stage before they "grow into deficit". To address these issues in the Dutch context, the Dutch educational system and YHC are summarized below.

\section{The Dutch educational system}

In the Netherlands most children attend a playgroup when they are two and a half years old and primary school at the age of 4 , but it is compulsory to attend fulltime education from the age of 5 until the end of the school year in which they turn 16 years. From the age of 16 education is partially compulsory, meaning that students must attend education for at least two days a week until they are 18 years old or until they obtain a basic qualification $(33,34)$. The compulsory education department of the municipalities is involved in monitoring truancy and school dropout. The department is also monitoring whether 18 to 23 year-old inhabitants obtain their basic qualification. For pupils with an IQ below 70, who attend a school for special needs, compulsory education ends with 16 years provided that they have a useful way of spending their days.

In primary and secondary schools, pupils are assessed annually by a team of teachers who determine whether they advanced enough to move on to the next grade. Otherwise, pupils are kept down a year. Gifted children can sometimes skip an entire year, mostly in primary school. A school year lasts from August 1st until July 31.

The Dutch educational system differs from the other countries in that there are already several difficulty levels in secondary school $(35,36)$. Most primary schools administer an aptitude test called CITO test in the last grade (group 8). Together with the advice of the primary school teacher this results in a choice for a secondary school that is adapted to the academic performance and intelligence of the pupil. The various levels of secondary education are:

- VMBO-BB (preparatory vocational secondary education, vocational training)

- VMBO-KL (an equal amount of theoretical education and vocational training)

- VMBO-GL (mixed learning path, between VMBO-KL and VMBO-TL)

- VMBO-TL (theoretical education)

- HAVO (higher general secondary education)

- VWO (pre-university education) 
After finishing VMBO, there are 4 more levels of $\mathrm{MBO}$ (intermediate vocational education) all offering a school-based education and an apprenticeship education. Higher education in the Netherlands is offered at colleges (HBO, higher professional education) or universities (WO, academic) for students who finished HAVO and VWO respectively.

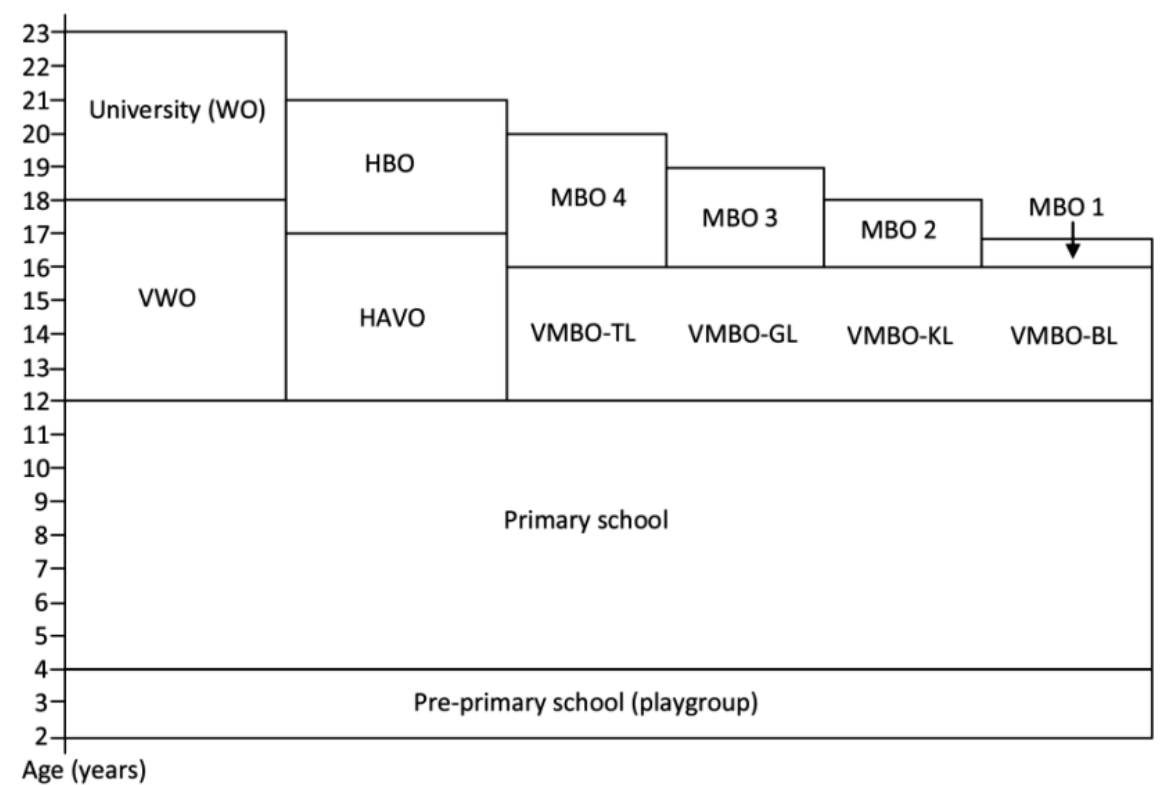

Figure 2. The Dutch educational system

\section{The Dutch youth health care}

As defined by the Public Health Act, The Dutch YHC aims to contribute to a healthy and secure childhood for all children aged 0-18 years, living in the Netherlands (37). The YHC provides preventive care and is closely cooperating with primary and secondary schools. In the Netherlands, the Community Paediatric Services consist of an infant welfare centre for children aged 0-4 years and a YHC Division from the Municipal Health Centre for children aged 4-18 years. Increasingly, this care is offered by one organisation for YHC for children aged 0-18 years. YHC doctors and specialized nurses offer routine health examinations and anticipatory guidance, free of charge, to all children between birth and 19 years $(38,39)$. For each child, a medical file is initiated at the infant welfare centre, which is transferred to the YHC Division of the Public Health Service at the age of 4. Although most of the health information is reported systematically and in a standardized way, some of the information is only included in open comments. Relevant variables that are recorded in the medical files are: hereditary diseases in mother or father's family, birth characteristics, diseases, surgeries, examinations, procedures or injuries, psychological symptoms and diseases. Furthermore, physician and specialist contacts, 
hospital admissions, developmental characteristics, personality, learning difficulties, educational level, family characteristics and SES of the parents.

YHC focuses on possibilities rather than on limitations, and embraces the new concept of positive health in which the ability to adapt and self-manage are crucial. In positive health, functioning is seen in the light of the physical, emotional and social challenges of life (40). Being able to participate in school and society is thus important to good health. YHC professionals work from a biopsychosocial (BPS) perspective and observe children from a broad holistic, integrative view. The BPS model (Figure 3) studies the interaction between biological, psychological and social factors to understand health and illness. Furthermore, the BPS model is systemic and regards a child's development as part of its whole system. The different factors are interrelated and changing one factor influences the others. In this thesis the BPS model is used as a basic theoretical framework.

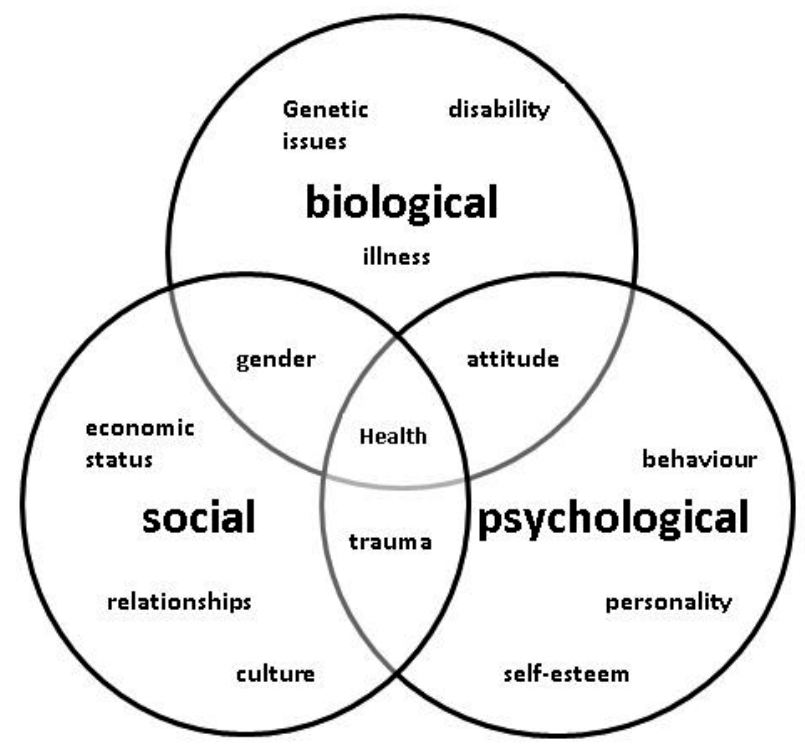

Figure 3. The biopsychosocial model

Furthermore, the structure of the International Classification of Functioning, disability and health for Children and Youth (ICF-CY) (Figure 4) is used to categorize the large number of candidate-determinants from the $\mathrm{YHC}$ files and to prevent us from forgetting relevant ones. The ICF-CY is based on the BPS model and is developed to describe a child's functioning and participation in basic areas and roles of social life, taken into account the health status, personal and environmental factors. It is a widely used universal tool that can be used in different cultures and is suitable for communication between various professional groups $(41,42)$. 

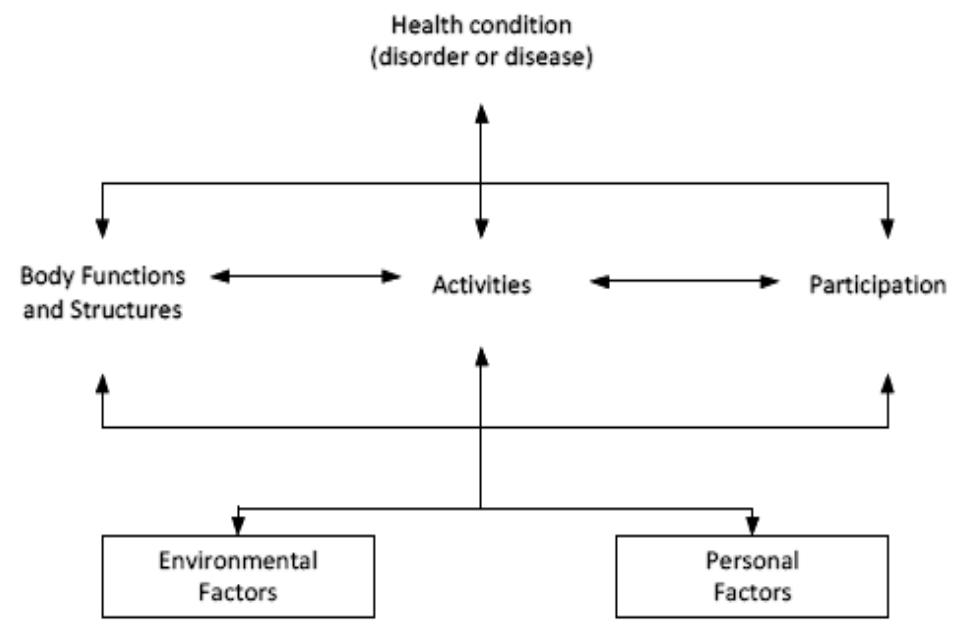

C Copyright Policy - open-access License

Figure 4. The international classification of Functioning and Health (ICF/ICF-CY), from the World Health Organization.

\section{Stay in or drop out (SIODO)}

The SIODO study uses a life-course perspective, the ICF-CY model to group the determinants and a mixed-methods design. It examines the biopsychosocial determinants related to school dropout and contributes to the development of a future tool that can be used in public health practice to tackle the problem of school dropout at its roots.

\section{Research questions}

In this thesis, the following research questions are addressed:

1. What biopsychosocial determinants in infancy, early and middle childhood, and adolescence predict school drop-out in young adulthood (18-23 years)?

2. Are gender beliefs and self-reported masculinity and femininity, beyond the influences of sex, related to school dropout in young adulthood?

3. Are adolescent low control beliefs, independent of parental socioeconomic background, related to school dropout and poor health in young adulthood?

4. How do young adults who graduated with a delay or dropped out of school perceive their life history and particularly their time in school?

5. How do parents, stakeholders from education and stakeholders from health care view the reasons for dropping out of school and their own and each other's roles in preventing school dropout at an early age? 


\section{Study design}

Figure 5 shows the study design of the SIODO study, a sequential mixed methods study where a quantitative case-control study with questionnaires and information from the YHC files was followed by and input for a qualitative study with semi structured interviews and homogenous focus groups.

In November 2011, the municipal compulsory education department of the city of Eindhoven (the Netherlands), selected all young adults aged 18-23 years who had not yet met the Dutch minimum educational requirement at the start of the 2010-11 school year. For the case-control study, they were sent a self-administered questionnaire and 660 youngsters were included. Half of them dropped out of school during the schoolyear 2010-11 (cases) and the other half still attended school at the end of that school year (controls). The case-control study provides insight in the contribution of SES, gender and the biopsychosocial determinants in early life, to school dropout in young adulthood. These determinants are discussed in individual interviews with youngsters, then aged 22 to 25 years, both dropouts and controls from the SIODO casecontrol study. From the perspective of the youngsters, we aim to gain an in-depth understanding of the biopsychosocial processes that lead to school dropout. The results from the case-control study and the individual interviews are discussed in the subsequent focus groups with parents, educational and health care professionals. In each focus group, the results from the previous focus groups are discussed. This enables us to further interpret and understand the acquired information. Furthermore, we will discuss what is needed to prevent school dropout and health-related problems with all stakeholders (youngsters in the interviews, parents, educational and health care professionals in the focus groups). In this mixed methods study, the quantitative and the qualitative studies are complementary and provide insights into the pathways leading to school dropout from different perspectives. Moreover, the qualitative findings may clarify issues raised in the quantitative case-control study. 


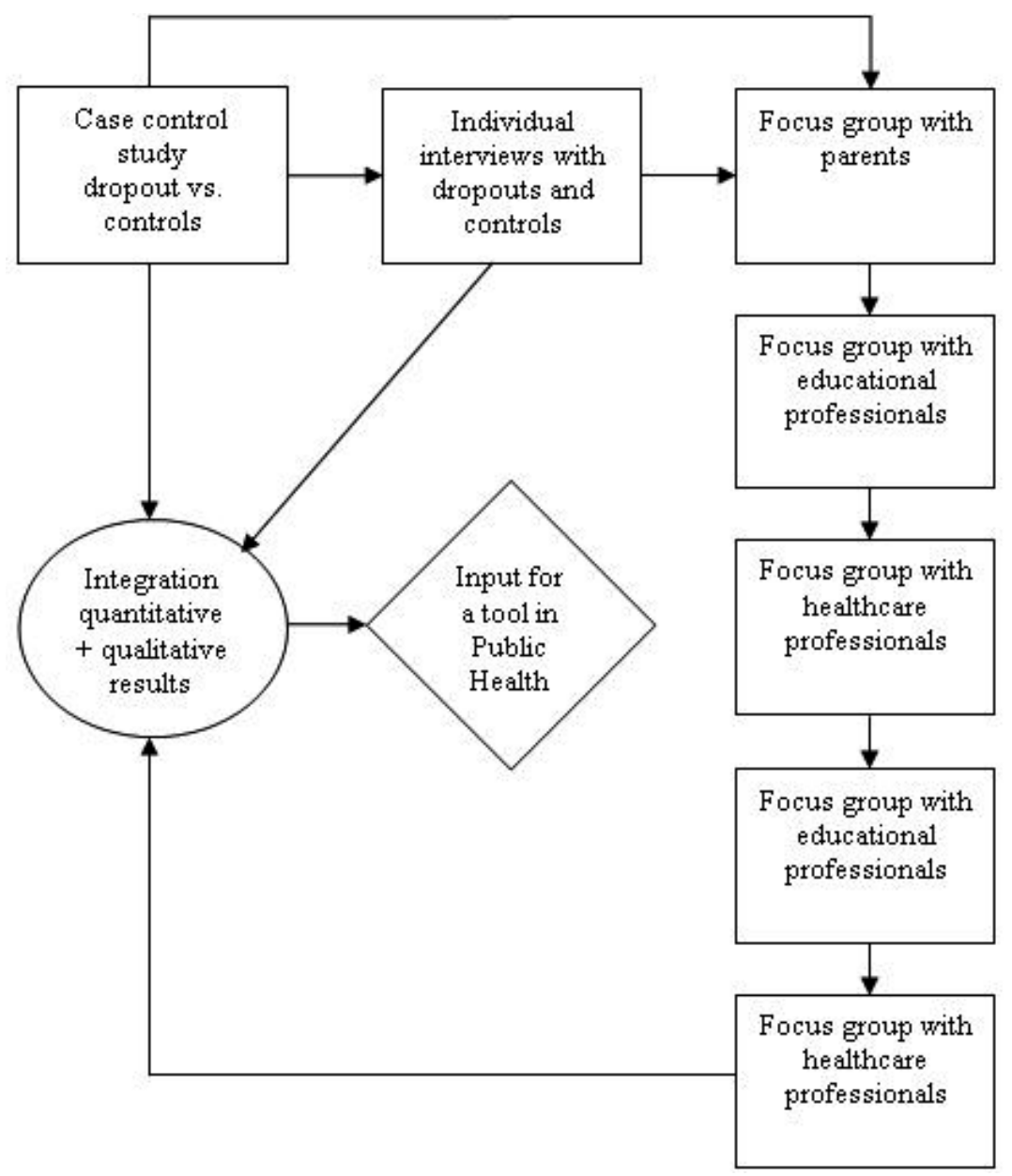

Figure 5. SIODO study design

A case-control study is followed by and input for the individual interviews. Subsequently, the results of the case-control study and the individual interviews are discussed in five sequential focus groups, each informed by the results of the previous ones. Integration of quantitative results and qualitative findings will provide input for a future tool to prevent school dropout.

\section{Outline of this thesis}

The chapters in this thesis will follow the structure of the SIODO study (Figure 5). In other words, the results from the studies described in chapter 3 to 5 are input for the study presented in chapter 6 and the studies described in chapters 3 to 6 provide input for the study described in chapter 7. 
Chapter 2 presents the design of the SIODO study, a mixed methods study where a quantitative case-control study is followed by and input for a qualitative study with interviews and focus groups. The strengths and limitation of this study are discussed, just as the role of YHC in signalling children at risk of school dropout.

Chapter 3 focuses on the data from the YHC files to answer the question "What biopsychosocial determinants in infancy, early and middle childhood, and adolescence predict school drop-out in young adulthood (18-23 years)?" This study aims to find determinants of increased risks of school dropout as early as possible which will eventually help the YHC professionals in reducing these risks. In the SIODO case-control design, data from the PPPC files, complemented by an additional questionnaire are used.

Chapter 4 elaborates on the relation between sex, gender and school dropout. To answer the question "Are gender beliefs and self-reported masculinity and femininity, beyond the influences of sex, related to school dropout in young adulthood?" we use data from the SIODO case-control study. Respondents fill out a self-administered questionnaire and both young men and women rate their own masculinity and femininity. Furthermore they answer questions about their gender beliefs.

Chapter 5 addresses the question "Are adolescent low control beliefs, independent of parental socioeconomic background, related to school dropout and poor health in young adulthood?" This study explores the mechanism of indirect selection on school dropout and poor health. We use data from the SIODO case-control study, in which the respondents fill out a questionnaire asking for current health status, recalled socioeconomic and social background, and recalled control beliefs (e.g. mastery and general selfefficacy).

Chapter 6 describes the findings from the semi-structured interviews with 11 young men and 14 young women, aged 22 to 25 years, who in 2011 were school dropouts or graduated with a delay. The aim of this qualitative study is to gain an in-depth understanding into the processes underneath our previous case-control findings that lead to school dropout from the perspective of the young adults. In this chapter, the young adults look back and reflect on their interactions with parents, peers and teachers from the moment in their youth that they can still remember until now. The interviews address the following research question: "How do young adults who graduated with a delay or dropped out of school perceive their life history and particularly their time in school?" Results are input for chapter 7.

Chapter 7 presents the findings of five homogenous focus group interviews with parents, educational professionals and youth (health) care professionals respectively. These stakeholders reflect on the previous results of the SIODO study, their view on the process 


\section{CHAPTER 1}

of school dropout and each other's role therein. On the basis of fictitious situations, the possibilities for the development of a prevention tool are explored. An answer is provided on the following research questions: "How do parents, stakeholders from education and stakeholders from health care view the reasons for dropping out of school and their own and each other's roles in preventing school dropout at an early age?"

Chapter 8 summarizes and discusses the main research findings. The quantitative and qualitative results will be integrated and the possibilities for a future tool to prevent school dropout from a public health perspective will be discussed. Finally, recommendations for future research and for practice and policy will be offered. 


\section{References}

1. OECD. The Organisation for Economic Co-operation and Development. 2008; Available from: www.oecd.org.

2. Lochner L, Moretti E. The Effect of Education on Crime: Evidence from Prison Inmates, Arrests, and SelfReports. American Economic Review. 2004;94(1):155-89.

3. Freudenberg N, Ruglis J. Reframing school dropout as a public health issue. Prev Chronic Dis. 2007 Oct;4(4):1-11.

4. Eurydice. Compulsory education in Europe 2014/2015: European Commission2015.

5. United Nations Educational Scientific and Cultural Organization (UNESCO). The revised International Standard Classification of Education (ISCED 2011). Paris 5 September 2011.

6. De Witte K, Cabus S, Groot W, Maassen van den Brink H. Voortijdig schoolverlaten. Rapportage van TIER/platform31: TIER-Maastricht university2014.

7. De Witte K, Cabus SJ. Dropout prevention measures in the Netherlands, an evaluation TIER-Maastricht University2010.

8. Molla M, Madans J, Wagener D. Differentials in adult mortality and activity limitation by years of education in the United States at the end of the 1990s. Population and Development Review. 2004;30:625-46.

9. Mackenbach JP. Health inequalities: Europe in profile. London: COI.2006.

10. Crimmins EM, Saito Y. Trends in healthy life expectancy in the United States, 1970, Äi1990: gender, racial, and educational differences. Social Science \&amp; Medicine. 2001;52(11):1629-41.

11. Droomers $P$, Limburg $L$, Westert $G$. Het terugdringen van sociaal economische verschillen in gezondheid tussen 2000 en 2020. Inhoud en organisatie van de SEGV monitor. RIVM rapport 260201001. Bilthoven: RIVM2003.

12. Townsend P, Davidson N. Inequalities in health: The Black report. Harmondswort: Penguin Books; 1982.

13. Pulkki L, Kivimäki M, Keltikangas-Järvinen L, Elovainio M, Leino M, Viikari J. Contribution of adolescent and early adult personality to the inverse association between education and cardiovascular risk behaviours: prospective population-based cohort study. International Journal of Epidemiology. 2003 December 1, 2003;32(6):968-75.

14. Women and Gender in Research. European Commision. Research \& Innovation - Sience in Society; [cited 201317 November]; Available from: http://ec.europa.eu/research/science-society/index.cfm?fuse action=public.topic\&id=1253\&lang=1.

15. Oliffe JL, Greaves LJ. Designing and Conducting Gender, Sex, and Health Research. Thousand oaks CA: Sage Publishers 2012.

16. Howard JA, Hollander J. Gendered situations, gendered selves. Oxford: AltaMira Press; 2000.

17. Ministery of Education Culture and Science. Dossier aanval op schooluitval [Drive to Reduce Drop-out Rates]. 2008 [cited 201328 March]; Available from: http://www.aanvalopschooluitval.nl/beleid/aanvalop-schooluitval-2008-2011.

18. Merens A, Hermans B. Emancipatiemonitor 2008. Den Haag: SCP/CBS2009.

19. Kahn J. An introduction to masculinities. West Sussex-UK: Wiley-Blackwell; 2009.

20. Battin-Pearson S, Newcomb MD, Abbott RD, Hill KG, Catalano RF, Hawkins JD. Predictors of early high school dropout: A test of five theories. Journal of educational psychology. 2000;92(3):568-82.

21. Bussey K, Bandura A. Social cognitive theory of gender development and differentiation. Psychological Review. 1999;106(4):676-713.

22. Gillmore MR, Hawkins JD, Day LE, Catalano RF. Friendship and Deviance: New Evidence on an Old Controversy. The Journal of Early Adolescence. 1992 February 1, 1992;12(1):80-95.

23. Patterson GR, DeBaryshe BD, Ramsey E. A developmental perspective on antisocial behavior. American Psychologist. 1989;44(2):329-35.

24. Bradshaw CP, O'Brennan LM, McNeely CA. Core competencies and the prevention of school failure and early school leaving. New Directions for Child and Adolescent Development. 2008;2008(122):19-32. 


\section{CHAPTER 1}

25. Jimerson S, Egeland B, Scroufe L, Carlson B. A prospective longitudinal study of high school dropouts; Examining multiple predictors across development. J School Psychol 2000;38:525-49.

26. Herweijer L. Gestruikeld voor de start. De school verlaten zonder startkwalificatie. Den Haag: Sociaal en Cultureel Planbureau2008 26 Augustus.

27. Snyderman R, Langheier J. Prospective health care: the second transformation of medicine. Genome Biology. 2006;7(2):104.

28. Wolraich M. The classification of child and adolescent mental diagnoses in primary care: Diagnostic and statistical manual for primary care (DSM-PC) child and adolescent version. Elk Grove Village, IL: American Academy of Pediatrics; 1996.

29. American Psychiatric Association. Diagnostic and statistical manual of mental disorder. 4 ed. Washington, DC2000.

30. Committee on Psychosocial Aspects of Child and Family Health and Task Force on Mental Health. Policy statement - The future of pediatrics: mental health competencies for pediatric primary care. Pediatrics. 2009;124(1):410-21.

31. Burns BJ, Costello EJ, Angold A, Tweed D, Stangl D, Farmer EM, et al. Children's mental health service use across service sectors. Health Affairs. 1995 August 1, 1995;14(3):147-59.

32. Doove B, Heller J, Feron F. JGZ op de drempel naar gepersonaliseerde zorg. TSG. 2013;91 (7):366-7.

33. Government of the Netherlands. Compulsory education. 2015 [cited 201522 November]; Available from: https://www.rijksoverheid.nl/onderwerpen/leerplicht/leerplicht-en-kwalificatieplicht

34. Netherlands Youth Institute. The Dutch education system. 2012 [cited 2015 23-11]; Available from: http://www.youthpolicy.nl/yp/Youth-Policy/Youth-Policy-subjects/Education-and-YouthUnemployment/The-Dutch-Education-System.

35. Nuffic E-. Het Amerikaanse onderwijssysteem beschreven en vergeleken met het Nederlandse [The American educational system compared with the Dutch one]. 2015 [cited 2015 23-11-2015]; 2:[Available from: https://www.epnuffic.nl/documentatie/publicaties/onderwijssysteem-verenigde-staten.pdf.

36. EP-Nuffic. Het onderwijssysteem van Nederland beschreven [The Dutch educational system]. 2014 [updated December; cited 2015 23-11]; 4:[Available from: https://www.nuffic.nl/bibliotheek/ onderwijssysteem-nederland.pdf.

37. Transitiebureau Jeugd, (VWS, VenJ, VNG. Youth health care's new basic responsibilities. 2015 [cited 2015 22-11-2015]; Available from: https://vng.nl/onderwerpenindex/jeugd/ jeugdgezondheidszorg/publicaties/factsheet-nieuw-basispakket-jgz.

38. Brugman E, Reijneveld S, Verhulst F, Verloove-Vanhorick S. Identification and management of psychosocial problems by preventive child health care. Archives of pediatrics and adolescent medicine. 2001;155:462-9.

39. Wieske RC, Nijnuis MG, Carmiggelt BC, Wagenaar-Fischer MM, Boere-Boonekamp MM. Preventive youth health care in 11 European countries: an exploratory analysis. International Journal of Public Health. 2012;57(3):637-41.

40. Huber M. Towards a new, dynamic concept of health: Its operationalisation and use in public health and healthcare and in evaluating health effects of food. Maastricht: Maastricht University; 2014.

41. Lee AM. Using the ICF-CY to organise characteristics of children's functioning. Disability and Rehabilitation. 2011;33(7):605-16.

42. Osteras N, Brage S, Garratt A, Benth J, Natvig B, Gulbrandsen P. Functional ability in a population: normative survey data and reliability for the ICF based Norwegian Function Assessment Scale. BMC Public Health. 2007;7(1):278. 


\section{Chapter 2}

\section{The early identification of risk factors}

on the pathway to school dropout in the SIODO study: a sequential mixed-methods study

Published as:

Theunissen M-J, Griensven van I, Verdonk P, Feron F, Bosma H. The early identification of risk factors on the pathway to school dropout in the SIODO study: a sequential mixed-methods study. BMC Public Health. 2012;12(1):1033. 


\section{Abstract}

\section{Background}

School dropout is a persisting problem with major socioeconomic consequences. Although poor health probably contributes to pathways leading to school dropout and health is likely negatively affected by dropout, these issues are relatively absent on the public health agenda. This emphasises the importance of integrative research aimed at identifying children at risk for school dropout at an early stage, discovering how socioeconomic status and gender affect health-related pathways that lead to dropout and developing a prevention tool that can be used in public health services for youth.

\section{Methods/Design}

The SIODO study is a sequential mixed-methods study. A case-control study will be conducted among 18 to 24 year olds in the south of the Netherlands ( $n=580$ ). Data are currently being collected from compulsory education departments at municipalities (dropout data), regional public health services (developmental data from birth onwards) and an additional questionnaire has been sent to participants (e.g. personality data). Advanced analyses, including cluster and factor analyses, will be used to identify children at risk at an early stage. Using the quantitative data, we have planned individual interviews with participants and focus groups with important stakeholders such as parents, teachers and public health professionals. A thematic content analysis will be used to analyse the qualitative data.

\section{Discussion}

The SIODO study will use a life-course perspective, the ICF-CY model to group the determinants and a mixed-methods design. In this respect, the SIODO study is innovative because it both broadens and deepens the study of health-related determinants of school dropout. It examines how these determinants contribute to socioeconomic and gender differences in health and contributes to the development of a tool that can be used in public health practice to tackle the problem of school dropout at its roots. 


\section{Background}

Good education increases life expectancy, reduces the burden of illness, delays the consequences of aging, decreases unhealthy behaviours and reduces health inequalities (1). Some have even argued that investments in education may save more lives than advances in medical technologies (2). However, almost $14 \%$ of all 18 to 24 year olds in Europe finish their education without a certificate or attain only a low level of education that is of little use on the labour market (3). School dropout is likely to have important implications for the mental and physical health status of a dropout. Health and developmental problems might also be implicated in the pathways leading to dropout. Despite its likely embedding in the discussion on socioeconomic inequalities in health, the issue of school dropout has mostly been neglected on the agenda of public health researchers and practitioners. To develop effective interventions, integrative research is needed into school dropout, and particularly its health-related determinants along the life course.

Current evidence on determinants can be grouped into family-related determinants (e.g. living in a single-parent family) $(1,4,5)$, school-related determinants (e.g. little concern in school policies) $(1,4-8)$ and pupil-related determinants (e.g. life-styles and personality) $(1,5,9,10)$. This evidence is, however, fragmented and has not yet been considered in a more integrative approach that acknowledges the life-course and developmental pathways that lead to school dropout $(7,11)$. This study uses a life-course, integrative focus to assess these pathways and proposes a mixed-methods design in order to broaden and deepen the insight into these pathways. Including early life will also make it possible to identify determinants that can be used in interventions aimed at preventing young people from dropping out of school.

We particularly expect socioeconomic status, sex (male, female) and gender (masculinity, femininity) to be important fundamental determinants in this study $(1,5,12,13)$. Girls tend to graduate faster and with higher grades than boys $(4,14)$. Boys more often engage in deviant behaviours and are more likely to drop out of school than girls $(13,15)$. Deviant behaviours and dropping out of school might give boys respect from their peers, prove their masculinity, and make them feel 'too cool for school' (16-20). The risk of dropout among girls, on the other hand, may be more strongly related to the tendency to internalise problems involving temperamental inhibition and depressive symptoms (21-24). Poor socioeconomic conditions during a child's upbringing are another fundamental background characteristic that is related to school dropout $(12,13)$ as well as poor health in children and adolescents (25). Poor health may even prove to be an under-studied mediator in the intergenerational reproduction of socioeconomic differences (9). Furthermore, the fact that early school leavers often have a poor health status (26) emphasises the importance of addressing early health problems and school dropout in the generational and intergenerational reproduction of socioeconomic inequalities in health. Our study aims to provide detailed insight into how gender and socioeconomic status might set children on a pathway leading to school dropout. 
The aim of the SIODO (stay in or drop out) study is to provide insight into the lifecourse pathways leading towards school dropout and the socioeconomic and gender differences found in these pathways. Exploring early health conditions in socioeconomic, environmental, psychosocial and personality- and gender-related contexts will also provide input for developing a tool that can be used at an early stage to identify children that are at risk of dropping out of school. This article presents the design of the sIODO study.

\section{Methods/Design}

The SIODO study is a sequential mixed-methods study (Figure 1) (27). We designed an unmatched case-control study to quantitatively identify the risk factors related to school dropout. The results of this case-control study will provide input for a qualitative study with semi-structured interviews and homogenous focus groups. The results of the case-control study will also be discussed in the steering group, which consists of stakeholders from policy, practice and research, and with representatives from a youth organisation that provides support for school dropouts (expert group). Approval for conducting this study was granted by the Medical Ethics Committee of Maastricht University (METC 11-4-099, decision 22-08-2011).

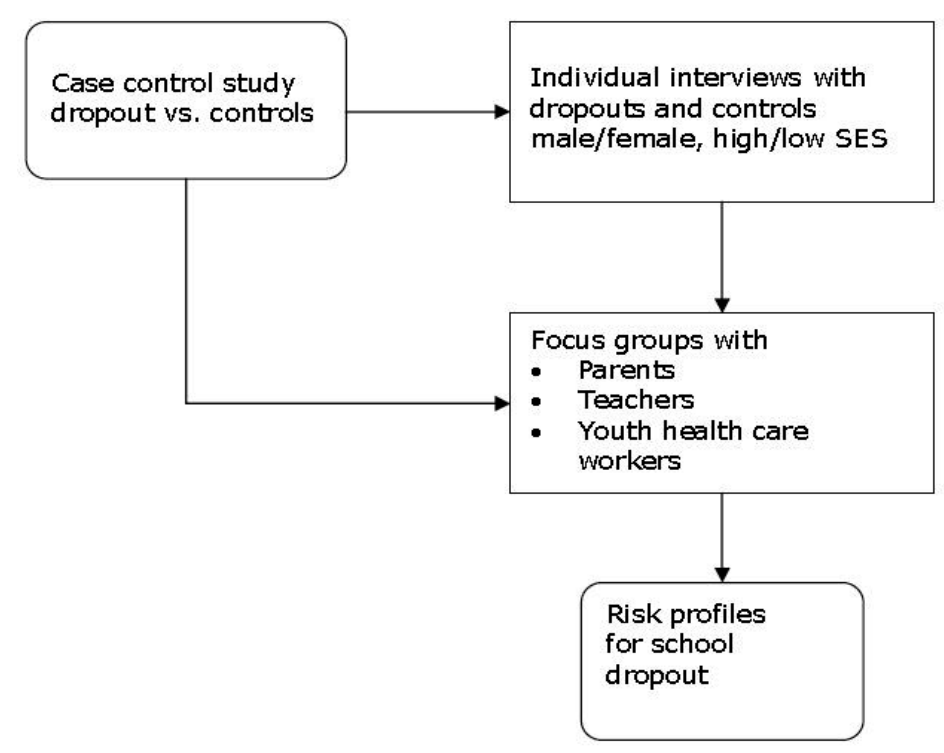

Figure 1. SIODO flowchart. The results of the case-control study will provide input for the individual interviews and for the focus groups, which in turn will help to understand the data and gain further insight into pathways leading to school dropout. The results from the individual interviews will be discussed in the focus groups. 


\section{Mixed-methods part 1: case-control study}

The case-control study compares school dropouts with a control group with regard to medical, developmental, socioeconomic, environmental, psychosocial and personality-, lifestyle- and gender-related determinants. School dropouts are defined as people aged 18-24 who have received lower secondary education or less and are no longer in education or training (28). They have had only a pre-primary, primary, lower secondary or short upper secondary education of less than two years $(4,29)$.

\section{Data collection}

\section{Compulsory education department}

The Compulsory Education Departments (CED) of Eindhoven and Maastricht provided names, addresses and other information about the school careers of eligible participants. This information, collected during education, is not susceptible to recall bias. Based on this information, students were divided in a case ('dropout') or control group.

\section{Preventive child \& youth healthcare files}

In the Netherlands, Preventive Child and Youth Healthcare (PCYH) ${ }^{1}$ doctors and specialised nurses offer routine health examinations and anticipatory guidance to all children between birth and 19 years $(30,31)$. They record the outcomes of the health examinations as well as information retrieved from different informants, such as parents, teachers and the young people themselves, in PCYH files. These files provide standardised longitudinal data on children's health, psychosocial circumstances and functioning. This data is not susceptible to recall bias.

\section{Questionnaire}

The questionnaire will provide additional data on past and current health status, socioeconomic status, life events $(32,33)$, gender $(34,35)$ and personality traits such as rebelliousness $(36,37)$, perceived control $(38,39)$, neuroticism and extraversion $(40,41)$ and social adequacy $(42,43)$. Personality traits are fairly stable and we assume that personality is not altered by school dropout. Because the last routine PCYH examination is given at the age of 14 , the questionnaire refers to the age of 16 for substance use and behaviour.

\footnotetext{
${ }^{1} \mathrm{PCYH}$ and $\mathrm{YHC}$ are different names of the same preventive youth health care (page 5).
} 


\section{Sample size calculation}

The power calculation for a retrospective study with a dichotomous outcome variable indicates that 290 cases would yield an $80 \%$ power to detect an odds ratio of 1.75 at a $\alpha$-level of $5 \%$ for an exposure of 0.2 and a ratio of cases to controls of $1(44,45)$. In total, 580 participants need to be included: 290 cases and 290 controls. It is estimated that four times that number need to be contacted in order to collect information on 580 participants. Hence, approximately 2300 young adults will be contacted.

\section{Study population}

\section{Setting}

In November 2011, the CED selected all eligible young adults aged 18-24 years who had received only lower secondary education or less and lived in Eindhoven. We performed a random sample among participants who had dropped out of school during the 20102011 school year (cases) and participants who still attended school or had graduated from at least upper secondary education during or at the end of the 2010-2011 school year (controls). We excluded cognitively impaired young people $(I Q<70)$, young people exempt from compulsory school attendance and young people with a lack of history in the PCYH files. In September 2012, we expanded the region to Maastricht and the villages around Eindhoven to enlarge the sample. The cities of Eindhoven and Maastricht are both located in the south of the Netherlands and have approximately 216,000 and 120,000 inhabitants, respectively (46).

\section{Recruitment}

We sent a paper questionnaire with study information and an informed consent to the selected participants. In addition, we asked for permission to approach them for an interview. Two reminders were sent. The second reminder included an internet questionnaire. Once we have expanded the region, selected participants will only receive a letter with study information and details for completing the online questionnaire. They will again be sent two reminders. More extensive information and details about the required consent can be found on the website. As an incentive, a raffle will be held among participants who fill in the questionnaire. They will receive cinema tickets. Young adults who do not return the questionnaire will be excluded from participation, but will be included in the non-responders analysis, which provides basic demographic information on the non-responders, such as age, sex and socioeconomic status. 


\section{Statistical analyses}

We will organise the information from the PCYH files with the International Classification of Functioning, Disability and Health for Children and Youth (ICF-CY) (Table 1) (47) and divide it over five timeframes. This is in line with the structure of the Dutch school system for early childhood (0-4 y and 4-8 y), middle childhood (8-12 y) and adolescence (12-16 y and 16-20 y) education (15). The routine health examinations conducted as part of the Dutch PCYH system take place at regular set times and key moments in children's development (31). The ICF-CY provides a multi-perspective approach and emphasises the interactive nature between domains $(47,48)$. We will translate the combinations of and interactions between these domains into risk profiles for the early identification of school dropout.

Table 1 Determinants from the PCYH files in the ICF-CY model

\begin{tabular}{|c|c|}
\hline 1. Disorder / Disease & 4.Participation \\
\hline Congenital abnormality & All-day childcare \\
\hline Physical illness & School \\
\hline Mental illness & Sports / Club \\
\hline Learning disability & Friends / Relationships \\
\hline Pregnancy & Work \\
\hline 2. Symptoms* & 5. Environmental factors \\
\hline Internalising / Externalising behaviour & Ethnicity \\
\hline Somatic complaints & Pregnancy / Childbirth \\
\hline Social problems & Family composition \\
\hline Sleeping difficulties & Parent-child relationship \\
\hline Eating difficulties & Health of parents / siblings \\
\hline Concentration difficulties & Parental education / profession \\
\hline Learning difficulties & Social environment \\
\hline Enuresis / Encopresis & Life event \\
\hline 3. Development* & Child abuse \\
\hline Growth & Bullying (victim) \\
\hline Motor & 6. Personal factors \\
\hline Speech / Language & Sex \\
\hline Cognitive & Neonatal period \\
\hline Social & Lifestyle \\
\hline Sexual & Personality \\
\hline
\end{tabular}

*The ICF-CY model has been adapted for a better match with the life-course determinants from the youth health care files. 'Activity' was changed to 'Development', which indicates age-appropriate abilities in a lifecourse perspective. 
We will use logistic analysis to relate potential predictors (disorders, symptoms, developmental, participatory, environmental and personal factors) to subsequent dropout. We will first conduct hierarchical analyses and separate checks on background characteristics such as gender and parental socioeconomic status to obtain insight into pathways that lead to dropout. This information reveals more recent (intermediate) characteristics in young adolescence such as developing disorders and symptoms. The focus on pathways will enable us to discover whether and how strongly adversities in young adulthood (e.g. hospital visits) affect school dropout, independent of the background characteristics during early life. Secondly, we examine profiles that combine life-course determinants to provide input for a tool to help identify risk profiles that may help predict pathways leading to school dropout. We use advanced techniques that allow the detection of optimal prediction models. These vary from a multiplicative interaction model (used for a stepwise approach) to advanced cluster and factor analyses. We will apply methods based on comparing areas under the curve (ROC) to find the profile and characteristics combinations with the best sensitivity and specificity, regarding the prediction of dropout. Depending on the measurement level of the variables, we use chi ${ }^{2}$ tests (categorical variable) or t-tests (continuous) to compare the demographic and socioeconomic characteristics of the responders and non-responders.

\section{Mixed methods part 2: qualitative study}

This study will conduct semi-structured interviews using a topic list based on literature and findings from the case-control study to understand the lives of the young men and women participating. These young people, both cases and controls, who all have different socioeconomic backgrounds, will be interviewed until no new information emerges (theoretical saturation).This will maximise the depth and richness of the data and increase the validity of the results (49). Marginally or less socially acceptable views are best assessed in individual interviews (49) and will therefore be used to explore the participants normative beliefs about gendered interaction with peers, parents and teachers. We will also stratify socioeconomic status. Male and female interviewers may interact differently with male and female participants and for this reason all four dyads will be included (50-52). We will record and transcribe the interviews for analysis $(53,54)$. Interim analyses will be conducted by starting the analytical process during data collection, which will allow us to go back and refine questions, develop hypotheses and explore these in more depth (55). We will furthermore read the data to identify emergent as well as anticipated themes and categories (56).

We will conduct six homogenous focus group sessions consisting of six to twelve people to explore a wide range of views held by parents and stakeholders (49). A moderator will use an interview protocol based on the health-related determinant model for school dropout (57). The focus group discussions aim to validate whether the themes 
found are recognisable. Also, they will explore possibilities for a tool to identify risk profiles for school dropout in the daily practice of public health (58). The focus groups (which take approximately 90-120 minutes) will take place in the following order: (1) parents, (2) teachers and (3) PCYH professionals who have experience with the youths in the control group. We chose this order because it reflects the order of problem solving dimensions in daily practice. Next we will hold focus groups with (4) parents, (5) teachers and (6) $\mathrm{PCYH}$ professionals who have experience with the youth in the case group. This way information from previous interviews can be presented to the next groups (55). Earlier findings and insights will be discussed in these last three focus groups with the aim of learning which issues were overlooked and which tools may be useful and feasible. We will record and transcribe the focus group interviews and an observer will take notes to describe the context and flow of the interview.

\section{Study population}

For the interviews, we will make a selection based on the sex and socioeconomic status of the questionnaire responders (both cases and controls) who consented to being approached for an interview.

For the focus groups, we will ask the participants' permission to contact their parents. We will guarantee heterogeneity by purposive sampling (59). Teachers and PCYH professionals will be recruited through the existing network and snowball sampling. All travel expenses will be refunded.

\section{Qualitative analyses of statistical analysis}

All interviews will be tape-recorded and transcribed verbatim and notes will be taken on the content of the individual interviews. We will read the notes and transcripts to gain a sense of the depth of the data and to collect and discuss ideas. We will apply researcher triangulation to base data collection, coding and analytic decisions on convergent validation (60). We will analyse the transcripts thematically to identify and report patterns and categories in the data $(49,61)$. After organising the data into meaningful groups, we will construct themes by combining codes to overarching categories (axial coding). To validate the findings with other stakeholders (method triangulation), we will discuss these themes in the focus groups. The group is the unit of analysis. We will test the validity of the findings by comparing the responses given in the focus groups as well as the findings from the other methods of data collection (data, researcher and method triangulation). 


\section{Discussion}

The SIODO study aims to contribute to the reduction of school dropout and socioeconomic health differences using an integrative approach that is both broadening and deepening. This study has several unique characteristics. First, the case-control trial will be followed by a qualitative study with the aim of further interpreting and understanding the acquired information. Second, the determinants of school dropout will be viewed from a life-course perspective. The PCYH files providing longitudinal data from birth to adolescence will enable us to gain insight into the life-course pathways leading to school dropout. This information is not affected by retrospective bias. Third, all of the stakeholders involved will participate, including the young adults and their parents. This will create a better understanding of the pathways leading to dropout and will contribute to a prevention tool. PCYH professionals working in the field of school dropout and unauthorised school absence will be able to learn from the valuable experiences shared by the dropouts, their parents and professionals in other fields. Fourth, this study will explore self-assigned masculine and feminine characteristics and gendered beliefs and their association with dropout in more depth. The subject of gender is still underrepresented in public health research. Fifth, our study will specifically address socioeconomic status and its relation to school dropout and health. This study will particularly provide more in-depth information on how health and developmental problems in early life contribute to adolescent school dropout, thus adding to the knowledge about the under-studied selection perspectives on the (persisting and widening) socioeconomic inequalities in health. Finally, the availability of information on the non-responders in the PCYH files allows for a detailed analysis of possible selection biases.

The PCYH system is well-suited for detecting and monitoring youths and families at risk for dropout (62). Rather than looking at early life symptoms from a dichotomous perspective (poor, good), we will assess symptoms that vary along a continuum from normal variations to problems, and finally disorders (with diagnoses) (15). The focus on symptoms, rather than on diagnoses, may require a shift in the current medical model, in which therapy is only followed after a diagnosis has been given. Even without a diagnosis, certain symptoms have a great impact on the child's development and quality of life $(15,63)$. School dropout is not an abrupt event, but a gradual process that often begins early in life. It is important that Dutch PCYH professionals, who offer routine health examinations and anticipatory guidance from birth, monitor social participation at each consultation, because joining sports, playing with friends or going to school is considered important for a healthy development. As school dropout has such a profound impact on an adolescent's life and increases the risks of social exclusion, it is important that PCYH professionals are able to detect and monitor youths and families at risk for dropout, also in cases where no disorder has been diagnosed. SIODO aims to contribute to effective early childhood interventions for preventing young people from dropping out of school. 
This study also has some limitations that need to be addressed. First, the data described in the PCYH files were not collected for the purpose of this study, so important information might be missing. However, the information in the PCYH files is not retrospectively biased, which means that this disadvantage is also one of the study's strengths. Secondly, not all data in the PCYH files will be complete and these files will have been compiled by many different PCYH professionals, each with their own interpretations and descriptions of certain situations, symptoms and life-course determinants. However, because the PCYH professionals work with this information in their daily practice, the ecological validity of our findings will likely be high and provide added value for developing the prevention tool. The collaboration between research, practice and policy will certainly enhance the study's use for optimising daily practice. Finally, participants in both the case and control groups had already been brought under the attention of the municipal CED. This may be why we had difficulties including participants who returned the completed questionnaire. We therefore included another Dutch region to increase the number of participants. For financial reasons, we proceeded with online questionnaires only.

The SIODO study will use a life-course perspective, the ICF-CY model to group the determinants and a mixed-methods design. In this respect, the SIODO study is innovative because it both broadens and deepens the study of the health-related determinants of school dropout. It examines how these determinants contribute to socioeconomic and gender differences in health and contributes to the development of a tool that can be used in public health practice to tackle the problem of school dropout at its roots.

\section{Ethics}

Approval for conducting this study was granted by the Medical Ethics Committee of Maastricht University. 


\section{References}

1. Freudenberg N, Ruglis J: Reframing school dropout as a public health issue. Prev Chronic Dis 2007, 4(4):1-11.

2. Woolf SH, Johnson RE, Phillips RL, Philipsen M: Giving everyone the health of the educated: an examination of whether social change would save more lives than medical advances. Am J Public Health 2007, 97(4):679-683.

3 Europe2020indicators. http://epp.eurostat.ec.europa.eu/portal/page/portal/europe_2020_indicators/ headline_indicators.

4. Herwijer L: Gestruikeld over de start: de school verlaten zonder startkwalificatie. In Edited by Planbureau SeC.; 2008.

5. O'Higgins N, D'Amato M, Caroleo F, Barone A: Gone for good? Determinants of school dropout in southern italy. Giornale degli Economisti 2007, 66(2):207-245.

6. Archambault I, Janosz M, Morizot J, Pagani L: Adolescent behavioral, affective and cognitive engagement in school: relationship to dropout. J Sch Health 2009, 79:408-415.

7. Jimerson S, Egeland B, Scroufe L, Carlson B: A prospective longitudinal study of high school dropouts; Examining multiple predictors across development. J School Psychol 2000, 38:525-549.

8. Tyler J, Lofstrom M: Finishing high school: alternative pathways and dropout recovery. Future Child 2009, 19(1):77-103.

9. Breslau J: Health in childhood and adolescence and high school dropout, California dropout research project report. vol. 17. Davis: University of California; 2010.

10. Suhrcke M, de paz Nieves C: The impact of health and health behaviours on educational outcomes in high-income countries: a review of the evidence. Copenhagen: WHO Regional office for Europe; 2011.

11. Bradshaw CP, O'Brennan LM, McNeely CA: Core competencies and the prevention of school failure and early school leaving. New Dir Child Adolesc Dev 2008, 2008(122):19-32.

12. Burns BJ, Costello EJ, Angold A, Tweed D, Stangl D, Farmer EM, Erkanli A: Children's mental health service use across service sectors. Health Aff 1995, 14(3):147-159.

13. Committee on Psychosocial Aspects of Child and Family Health and Task Force on Mental Health: Policy statement - the future of pediatrics: mental health competencies for pediatric primary care. Pediatrics 2009, 124(1):410-421.

14. Merens A, Van den Brakel M, Hartgers M, Hermans B: Emancipatiemonitor 2010.: Sociaal en Cultureel Planbureau, Centraal Bureau voor de Statistiek; 2010.

15. Wolraich M: The classification of child and adolescent mental diagnoses in primary care: diagnostic and statistical manual for primary care (DSM-PC) child and adolescent version. Elk Grove Village, IL: American Academy of Pediatrics; 1996.

16. Alatupa S, Pulkki-Råback L, Hintsanen M, Mullola S: Childhood disruptive behaviour and school performance across comprehensive school: a prospective cohort study. Psych 2011, 2(6):542-551.

17. Bradshaw C, Schaeffer C, Petras H, lalongo N: Predicting negative life outcomes from early aggressive disruptive behavior trajectories: gender differences in maladaptation across life domains. J Youth Adolesc 2010, 39(8):953-966.

18. Chun H, Mobley M: Gender and grade-level comparisons in the structure of problem behaviors among adolescents. J Adolesc 2010, 33(1):197-207.

19. Ensminger $M$, Slusarcick $A$ : Paths to high school graduation or dropout: a longitudinal study of a first grade cohort. Social Educ 1992, 65:95-113.

20. Staff J, Kreager D: Too cool for school? peer status and high school dropout.; 2008.

21. Bayer J, Rapee R, Hiscock H, Ukoumunne O, Migalopoulos C, Wake M: Translational research to prevent internalizing problems in early childhood. Depress Anxiety 2011, 28(1):50-57.

22. Erath S, Flanagan K, Bierman K: Social anxiety and peer relations in early adolescence: behavioral and cognitive factors. J Abnorm Child Psychol 2007, 35(3):405-416. 
23. Needham BL: Adolescent depressive symptomatology and young adult educational attainment: an examination of gender differences. J Adolesc Health 2009, 45(2):179-186.

24. Rubin K, Coplan R, Bowker J: Social withdrawal in childhood. Annu Rev Psychol 2009, 60:141-171.

25. Mackenbach JP: Health inequalities: Europe in profile. London: COI; 2006.

26. The organisation for economic Co-operation and development. www.oecd.org.

27. Tashakkori A, Teddlie C: Handbook of mixed methods in social \& behavioural research. Thousand oaks: Sage; 2003.

28. European council (2003) council conclusions of 5 May 2003 on reference levels of European average performance in education and training (benchmarks). Off J Eur Union, C 134/03.

29. United Nations Educational Scientific and Cultural Organization (UNESCO): The revised International standard classification of education (ISCED 2011). 2011.

30. Kuo AA, Inkelas M, Lotstein DS, Samson KM, Schor EL, Halfon N: Rethinking well-child care in the United States: an international comparison. Pediatrics 2006, 118(4):1692-1702.

31. Platform jeugdgezondheidszorg: Richtlijn contactmomenten. Basistakenpakket jeugdgezondheidszorg 019 jaar. Richtlijn 1. 2003.

32. Nationale jeugdmonnitor. http://jeugdmonitor.cbs.nl.

33. Illnesses questionnaire. https://monitorgezondheid.nl.

34. Chu JY, Porche MV, Tolman DL: The adolescent masculinity ideology in relationships scale: development and validation of a New measure for boys. Men and Masculinities 2005, 8(1):93-115.

35. Tolman DL, Porche MV: The adolescent femininity ideology scale: development and validation of a new measure for girls. Psychol Women Q 2000, 24(4):365-376.

36. Klabbers $G$, Bosma $H$, van den Akker M, van Boxtel MPJ, Kempen GIJM, McDermott MR, Van Eijk JTM: Measuring rebelliousness and predicting health behaviour and outcomes. J Health Psychol 2009, 14(6):771-779.

37. McDermott MR: Rebelliousness. In M. J. Apter (Ed.), motivational styles in everyday life: a guide to reversal theory. Washington, DC: American PsychologicalAssociation; 2001:167-185.

38. Kempen GIJM, Van Heuvelen MJG, Van Sonderen E, Van den Brink RHS, Kooijman AC, Ormel J: The relationship of functional limitations to disability and the moderating effects of psychological attributes in community-dwelling older persons. Soc Sci Med 1999, 48:1161-1172.

39. Pearlin LI, Schooler C: The structure of coping. J Health Soc Behav 1978, 19(1):2-21.

40. Eysenck H, Eysenck S: The manual of the Eysenck personality questionnaire. London: Hodder and Stoughton; 1975.

41. Eysenck HJ, Eysenck SB: Manual of the Eysenck personality questionnaire: comprising the EPQ-revised (EPQ) and EPQ-R short scale EDITS. CA: San Diego; 1994.

42. Barelds DPH, Dijkstra P: Narcissistic personality inventory: structure of the adapted Dutch version. Scand J Psychol 2010, 51(2):132-138.

43. De Raad B, Barelds DPH: A new taxonomy of Dutch personality traits based on a comprehensive and unrestricted list of descriptors. J Pers Soc Psychol 2008, 94(2):347-364.

44. Elich M, Sinnema G: Chronische ziekten en lichamelijke handicap. In Handboek kinderen en adolescenten Houten. Edited by Bakker GA, Van Zeben-van-der DMCB, Dewispelaere AJ, et al.: Bohn, Stafleu, Van Loghum; 2010:75-95. 4.

45. Van de Mheen $\mathrm{H}$ : Inequalities in health to be continued? A life-course perspective on socio-economic inequalities in health. Ponsen \& Looijen: Wageningen; 1998.

46. Dutch population. http://statline.cbs.nl/StatWeb/publication/?VW=T\&DM=SLNL\&PA=70748NED\&D1= 0,2,4,16,18,20,22,24\&D2=a\&D3=0\&D4=a\&D5=I\&HD=090707-1905\&HDR=T\&STB=G4,G2,G1,G3.

47. Lee AM: Using the ICF-CY to organise characteristics of children's functioning. Disabil Rehabil 2011, 33(7):605-616.

48. Osteras N, Brage S, Garratt A, Benth J, Natvig B, Gulbrandsen P: Functional ability in a population: normative survey data and reliability for the ICF based norwegian function assessment scale. BMC Publ Health 2007, 7(1):278. 


\section{CHAPTER 2}

49. Green J, Thorogood N: Qualitative methods for health research. 2nd edition. Los Angeles: Sage Publications; 2009.

50. Brown S: What makes men talk about their health? J Gender Stud 2001, 10:187-195.

51. Oliffe J, Mróz L: Men interviewing men about health and illness: ten lessons learned. J Mens Health Gender 2005, 2:257-260.

52. Smith J, Braunack-Meyer A, Wittert G, Warin M: Qualities men value when communicating with general practitioners: implications for primary care settings. MJA 2008, 189(11-12):618-621.

53. Braun V, Clarke V: Using thematic analysis in psychology. Qual Res Psychol 2006, 3:77-101.

54. de Dierckx Casterlé B, Gastmans C, Bryon E, Denier Y: QUAGOL: A guide for qualitative analysis. Int J Nurs Stud 2011.

55. Pope C, Ziebland S, Mays N: Analysing qualitative data. BMJ 2000, 320(7227):114-116.

56. Ziebland S, McPherson A: Making sense of qualitative data analysis: an introduction with illustrations from DIPEx (personal experiences of health and illness). Med Educ 2006, 40(5):405-414.

57. The Health Communication Unit: Using focus groups, version 2.0.: University of Toronto; 2002. http://www.thcu.ca/infoandresources/publications/Focus_Groups_Master_Wkbk_Complete_v2_content _06.30.00_format_aug03.pdf.

58. Bender D, Ewbank D: The focus group as a tool for health research: issues in design and analysis. Health Transit Rev 1994, 4(1):63-79.

59. Wong L: Focus group discussion: a tool for health and medical research. Singapore Med J 2008, 49(3):256-261.

60. Polit J, Beck C: Nursing research. 8th edition. New Delhi: Williams and Wilkins; 2008.

61. Braun V, Clarke C: Using thematic analysis in psychology. Qual Res Psychol 2006, 3(2):77-101.

62. Inspectie voor de gezondheidszorg: Zorgverlening door de jeugdgezondheidszorg, GGZ en huisartsen aan Gessica vanuit het perspectief van een veilige ontwikkeling van het kind. Den Haag: Inspectie voor de gezondheidszorg; 2007.

63. American Psychiatric Association: Diagnostic and statistical manual of mental disorder. 4th edition. Washington, DC: 2000. 


\section{Appendix: \\ English translation of the questionnaires used in the SIODO-study}

\section{Appendix: \\ English translation of the questionnaires used in the SIODO-study}

\begin{tabular}{|c|c|}
\hline Determinant & Scale \\
\hline $\begin{array}{l}\text { General background -past/current } \\
\text { health status - Life events \& } \\
\text { substance use }\end{array}$ & $\begin{array}{l}\text { Illnesses questionnaire and National Youth Monitor of the Central Bureau } \\
\text { of Statistics Netherlands }(1,2)\end{array}$ \\
\hline Personality - rebelliousness & Social Reactivity Scale (Dutch version) $(3,4)$ \\
\hline Personality - perceived control & $\begin{array}{l}\text { Pearlin \& Schooler's Mastery Scale (Dutch version) }(5,6) \\
\text { General self-efficacy (Sherer) }(7,8)\end{array}$ \\
\hline Personality - social inadequacy & Dutch Personality Questionnaire (subscale) $(9,10)$ \\
\hline $\begin{array}{l}\text { Personality - neuroticism \& } \\
\text { extraversion }\end{array}$ & Personality Questionnaire of Eysenck $(11,12)$ \\
\hline $\begin{array}{l}\text { Masculinity beliefs perception/ } \\
\text { expressions of masculinity }\end{array}$ & $\begin{array}{l}\text { Adolescent Masculinity Ideology in relationships Scale (AMIRS; 12-item } \\
\text { subscale) (13) }\end{array}$ \\
\hline $\begin{array}{l}\text { Femininity beliefs -internalising of } \\
\text { feminine behaviours }\end{array}$ & $\begin{array}{l}\text { Adolescent Femininity Ideology Scale (AFIS; 10-item subscale "self in } \\
\text { relationship") (14) }\end{array}$ \\
\hline Symptoms of ADHD & Adult ADHD Self-Report Scale (ASRS-v1.1) Symptom Checklist, part A (15) \\
\hline
\end{tabular}

\section{General questions}

The general background contains questions like sex, date of birth, country of birth, postal code, parents' education, family composition and education level.

We ask about present and former diseases, life events and substance use on the age of 16 years.

\section{Rebelliousness}

1. When you are told that you are breaking a rule (for example 'No smoking') is your first reaction to ...

2. You have been treated badly by someone. Do you ... 
3. Doing physical exercise, you feel some pain. Do you...

4 'I enjoy the thrill I get from being difficult and awkward.' Do you ...

5 If people are unkind to you, do you feel you should be ...

6 Do you find it exciting to do something 'shocking'?

7 If you are asked particularly NOT to do something, do you feel an urge to do it?

8. You are joining a group of people drinking alcohol. You don't like alcoholic drinks, but they offer you one. What would you do?

9. Do you tease people unnecessarily just so as to have some fun at their expense?

10. A parking attendant tells you that you cannot park where you have just put the car. Would you ...

11. How often do you do something you shouldn't just to get some excitement?

12. You are asked to join an activity you actually dislike. What would you say?

13 If you get yelled at by someone in authority, would you ...

14 If a person your age was mean to you, would you ...

15. Can you image something you are definitely against?

16. A charity will not accept you as a volunteer. Is your first reaction to ...

17. How often do others say that you are a difficult person?

18. If you ask a person at a party to dance with you who says 'no' without offering any explanation, would you ...

\section{Perceived control}

\section{Strongly agree / agree / no disagreement-agreement / disagree / strongly} disagree

1. When I make plans, I am certain I can make them work.

2. One of my problems is that I cannot get down to work when I should.

3. If I can't do a job the first time I keep trying until I can.

4. I have confidence in my own abilities.

5. When I set important goals for myself, I rarely achieve them.

6. I avoid facing difficulties.

7. If something looks too complicated, I will not even bother to try it.

8. When I have something unpleasant to do, I stick to it until I finish it.

9. I do not seem capable of dealing with most problems that come up in life.

10. If I make a decision to do something, I will do it.

11. When trying to learn something new, I soon give up if I am not initially successful

12. When unexpected problems occur, I don't handle them well.

13. Failure just makes me try harder.

14. I avoid trying to learn new things when they look too difficult for me.

15. I feel insecure about my ability to do things.

16. I give up easily.

17. I have little control over the things that happen to me. 
18. There is really no way I can solve some of the problems I have.

19. There is little I can do to change many of the important things in my life.

20. I often feel helpless in dealing with the problems of life.

21. Sometimes I feel that I'm being pushed around in life.

22. What happens to me in the future mostly depends on me.

23. I can do almost everything, if I want to.

\section{Social inadequacy*}

\section{Yes / ? / No}

1. I find it tiresome to speak to a group of people that I do not know.

2. I find it tiresome to talk to people that I do not know.

3. If I will be meeting people, I get nervous.

4. I find it difficult to make contact with people that I do not know.

5. If possible, I avoid receptions.

6. In the company of people that I do not know, I am shy.

7. I find it tiresome to have many people around.

8. It is difficult for me to make new friends.

9. I like visitors more when they are leaving than when they are coming.

10. In a group of people, I rather stay in the background.

11. Only with people that I do know well, I feel comfortable.

12. I have only little influence on other people.

13. I rather work alone than with a number of people.

14. I have only little need for contact with other people.

15. If people look at me, I get shy.

* These items are not back-translated and can therefore only be seen and used in the context of this letter to BMC Public Health.

\section{Neuroticism \& extraversion}

Yes / No

1. Does your mood often go up and down?

2. Are you a talkative person?

3. Do you ever feel 'just miserable' for no reason?

4. Are you rather lively?

5. Are you an irritable person?

6. Do you enjoy meeting new people?

7. Are your feelings easily hurt?

8. Can you usually let yourself go and enjoy yourself at a lively party?

9. Do you often feel 'fed-up? 
10. Do you usually take the initiative in making new friends?

11 . Would you call yourself a nervous person?

12. Can you easily get some life into a rather dull party?

13. Are you a worrier?

14. Do you tend to keep in the background on social occasions?

15. Would you call yourself tense or 'highly-strung'?

16. Do you like mixing with people?

17. Do you worry too long after an embarrassing experience?

18. Do you like plenty of bustle and excitement around you?

19. Do you suffer from 'nerves'?

20. Are you mostly quiet when you are with other people?

21. Do you often feel lonely?

22. Do other people think of you as being very lively?

23. Are you often troubled about feelings of guilt?

24. Can you get a party going?

\section{AMIRS}

\section{Strongly disagree / disagree / agree / strongly agree}

1. It's important for a guy to act like nothing is wrong, even when something is bothering him

2. In a good dating relationship, the guy gets his way most of the time

3. I can respect a guy who backs down from a fight

4. It's ok for a guy to say no to sex

5. Guys should not let it show when their feelings are hurt

6. A guy never needs to hit another guy to get respect

7. If a guy tells people his worries, he will look weak

8. I think it's important for a guy to go after what he wants, even if it means hurting other people's feelings

9. I think it's important for a guy to act like he is sexually active even if he is not

10. I would like to be friends with a guy who is gay

11. It's embarrassing for a guy when he needs to ask for help

12. I think it's important for a guy to talk about his feelings, even if people might laugh at him 


\section{AFIS}

Strongly disagree / disagree / no disagreement-agreement / agree / strongly agree

1. I would tell a friend she looks nice, even if I think she shouldn't go out of the house dressed like that

2. I express my opinions only if I can think of a nice way of doing it

3. I worry that I make others feel bad if I am successful

4. I would not change the way I do things in order to please someone else

5. I tell my friends what I honestly think even when it is an unpopular idea

6. Often I look happy on the outside in order to please others, even if I don't feel happy on the inside

7. I wish I could say what I feel more often than I do

8. I feel like it's my fault when I have disagreements with my friends

9. When my friends ignore my feelings, I think that my feelings weren't very important anyway

10. I usually tell my friends when they hurt my feelings

\section{$A D H D$}

Never / rarely / sometimes / often / very often

1. How often do you have trouble wrapping up the final details of a project, once the challenging parts have been done?

2. How often do you have difficulty getting things in order when you have to do a task that requires organization?

3. How often do you have problems remembering appointments or obligations?

4. When you have a task that requires a lot of thought, how often do you avoid or delay getting started?

5. How often do you fidget or squirm with your hands or feet when you have to sit down for a long time?

6. How often do you feel overly active and compelled to do things, like you were driven by a motor? 


\section{CHAPTER 2}

\section{References}

1. Nationale jeugdmonitor [http://jeugdmonitor.cbs.nl]

2. IIInesses questionnaire [https://monitorgezondheid.nl]

3. Klabbers G, Bosma H, van den Akker M, van Boxtel MPJ, Kempen GIJM, McDermott MR, Van Eijk JTM: Measuring Rebelliousness and Predicting Health Behaviour and Outcomes. Journal of Health Psychology 2009, 14(6):771-779.

4. McDermott MR: Rebelliousness. In M. J. Apter (Ed.), Motivational styles in everyday life: A guide to reversal theory. Washington, DC:American PsychologicalAssociation 2001:167-185.

5. Kempen GIJM, Van Heuvelen MJG, Van Sonderen E, Van den Brink RHS, Kooijman AC, Ormel J: The relationship of functional limitations to disability and the moderating effects of psychological attributes in community-dwelling older persons. Social Science \& Medicine 1999, 48:1161-1172.

6. Pearlin LI, Schooler C: The Structure of Coping. Journal of Health and Social Behavior 1978, 19(1):2-21.

7. Sherer M., Maddux J. E., Mercandante B., Prentice-Dunn S., Jacobs B., \& Rogers R. W. The Self-Efficacy Scale: Construction and validation. Psychological Reports 1982, 51, 663-671.

8. Imam S.S.: Sherer et al. General self-efficacy scale: dimensionality, internal consistency, and temporal stability. International Islamic University Malaysia, Department of Psychology, May 2007.

9. Barelds DPH, Dijkstra P: Narcissistic Personality Inventory: Structure of the adapted Dutch version. Scandinavian Journal of Psychology 2010, 51(2):132-138.

10. De Raad B, Barelds DPH: A new taxonomy of Dutch personality traits based on a comprehensive and unrestricted list of descriptors. Journal of Personality and Social Psychology 2008, 94(2):347-364.

11. Eysenck H, Eysenck S: The Manual of the Eysenck Personality Questionnaire. London: Hodder and Stoughton; 1975.

12. Eysenck HJ, Eysenck SB: Manual of the Eysenck Personality Questionnaire: Comprising the EPQ-Revised (EPQ) and EPQ-R Short Scale EDITS. San Diego, CA; 1994.

13. Chu JY, Porche MV, Tolman DL: The Adolescent Masculinity Ideology in Relationships Scale: Development and Validation of a New Measure for Boys. Men and Masculinities 2005, 8(1):93-115.

14. Tolman DL, Porche MV: The Adolescent Femininity Ideology Scale: Development and validation of a new measure for girls. Psychology of Women Quarterly 2000, 24(4):365-376.

15. Adler LA, Newcorn JH: Administering and evaluating the results of the adult ADHD Self-Report Scale (ASRS) in adolescents. The Journal of clinical psychiatry 2011, 72 (6): e20 


\section{Chapter 3 \\ Why Wait? Early Determinants of School Dropout in Preventive Pediatric Primary Care}




\section{Abstract}

\section{Background}

To answer the question of what bio-psychosocial determinants in infancy, early and middle childhood, and adolescence predict school drop-out in young adulthood, we approached the complex process towards school dropout as a multidimensional, lifecourse phenomenon. The aim is to find signs of heightened risks of school dropout as early as possible which will eventually help public health workers in reducing these risks.

\section{Methods}

In a case-control design, we used data from both the Preventive Pediatric Primary Care (PPPC) files (that contain information from birth onwards) and additional questionnaires filled out by 529 youngsters, aged $18-23$ years, and living in the South-east of the Netherlands. We first conducted univariate logistic regression analyses with school-dropout as the dependent variable. Backward and forward stepwise analyses with the significant variables were done with variables pertaining to the 0 to 4 year period. Remaining significant variables were forced into the next model and subsequently variables pertaining to respectively the 4 to 8,8 to 12 and 12 to 16 year period were introduced in a stepwise analysis. All analyses were cross-validated in an exploratory and confirmatory random half of the sample.

\section{Results}

One parent families and families with a non-Western background less often attended the health examinations of the PPPC and such less attendance was related to school dropout. The birth of a sibling (OR $0.63,95 \% \mathrm{Cl} 0.43-0.93$ ) in infancy and self-efficacy (OR 0.53, 95\% Cl 0.38-0.74) in adolescence decreased the odds of school dropout; externalizing behavior ( $\mathrm{OR} 2.81,95 \% \mathrm{Cl}$ 1.53-5.14) in middle childhood and (sickness) absence (OR 5.62, 95\% Cl 2.18-14.52) in adolescence increased the risks.

\section{Conclusion}

To prevent school dropout, PPPC professionals should not wait until imminent dropout, but should identify and tackle risk factors as early as possible and actively approach youngsters who withdraw from public health care. 


\section{Introduction}

School dropout is an important but complex public health problem (1) that, when trying to tackle the problem, requires an integrative perspective and approach (2). It is strongly associated with poor health and has major adverse socioeconomic consequences on both the macro- and personal level (3). Dropout is not a discrete event, but a dynamic developmental process that often begins early in life $(4,5)$. Nevertheless, most interventions to prevent school dropout are school-based and targeted at factors that operate late in the dropout process $(6,7)$. Few interventions are aimed at tackling the problems at its roots by identifying children with physical, mental, psychosocial, or socioeconomic problems early in life. The aim of this study is to offer insight into the bio-psychosocial determinants of school dropout in infancy, early and middle childhood, and adolescence. This will contribute to develop more effective interventions in public health care aimed at reducing school dropout at an as early as possible stage.

Many factors that influence the process of early school leaving are already identified (8) and can be divided in student-related (e.g. overall poor health in childhood and adolescence (3), externalizing and internalizing behavior $(9,10)$, life-style, personality $(3,11,12)$, sex differences and gender beliefs (13), family-related (e.g. living in a singleparent family(14)) and school-related factors (e.g. little concern in school policies (15)). However, most studies until now are restricted to single determinants. Therefore, in the current study among Dutch young adolescents, we used a multidimensional approach with information from both the Preventive Pediatric Primary Care (PPPC) ${ }^{2}$ and an additional questionnaire. In The Netherlands, the PPPC offers longitudinal anticipatory guidance to all children between birth and 19 years $(16,17)$ enabling us to perceive the determinants of school dropout from a life-course perspective.

\footnotetext{
${ }^{2}$ PPPC and YHC are different names of the same preventive youth health care (page 5).
} 
Box 1: The Dutch youth health care system

In the Netherlands, PPPC doctors and specialized nurses (Preventive Child and Youth Healthcare) offer routine health examinations and anticipatory guidance, free of charge, to all children between birth and 19 years using a bio-psychosocial model

The aim of this study is to find determinants of increased risks of school dropout as early as possible which will eventually help public health workers in reducing these risks.

We used case-control data from the PPPC files and additional questionnaires from 529 Dutch youngsters to examine which bio-psychosocial determinants in infancy, childhood and adolescence predict school drop-out in young adulthood (18-23 years). An age-attentive stepwise modeling allowed for the identification of determinants as early in life as possible. This study has an explorative character and cross-validating the analyses in a random exploratory and confirmatory half of the sample minimized the possibility of capitalization on chance.

\section{Methods}

\section{Study population}

This study is part of the Dutch SIODO (Stay In Or Drop Out) study, a sequential mixedmethods study focusing on the early identification of risk factors on the pathway to school dropout (18). The current paper is based on the quantitative case-control data from SIODO in which school dropouts are compared with a control group with regard to medical, developmental, environmental, psychosocial and personality- and lifestylerelated determinants in infancy, childhood and adolescence. In November 2011, the municipal compulsory education department of the city of Eindhoven, The Netherlands selected all young adults aged 18 to 23 years who did not yet attain a basic qualification on August 1st, 2010, but who all still attended school at the start of that school year. This minimum qualification (for making a good entry on the labor market) is equivalent to higher general secondary education, pre-university education or intermediate vocational education, Level $2(19,20)$. At this age, generally young adults have obtained this required qualification. We excluded cognitively impaired young people $(I \mathrm{Q}<70)$ and young people exempt from compulsory school attendance. We divided the participants into those who dropped out of school during the 2010-2011 school year (cases) and those who still attended school or graduated and attained at least a basic qualification during or at the end of the 2010-2011 school year (controls). Cases and controls being so similar at the start of the 2010-2011 school year was supposed to avoid selection bias. 


\section{Recruitment}

All eligible youngsters were sent a self-administered questionnaire with an informed consent form. In addition, we asked for the respondents' permission to link the questionnaires to the PPPC files. A power analysis for the association between the experience of a chronic health condition in early life and the occurrence of early school leaving during adolescence indicated that 290 cases and 290 controls should be sufficient to yield an $80 \%$ power to detect an odds ratio of 1.75 at the conventional alpha level (5\%) for an exposure prevalence of 0.2. More controls than cases returned and filled in the questionnaire. We included all cases with a completed questionnaire and took a random sample from the control group with a completed questionnaire. Subsequently we requested their PPPC file from the Public Health Service. We eventually were able to obtain the PPPC files of 283 cases and 343 controls out of the participants who completed the questionnaire, of which 229 cases (80.9 \%) and 300 controls (89.5\%) had at least once visited the PPPC in every period of study (Figure 1). 


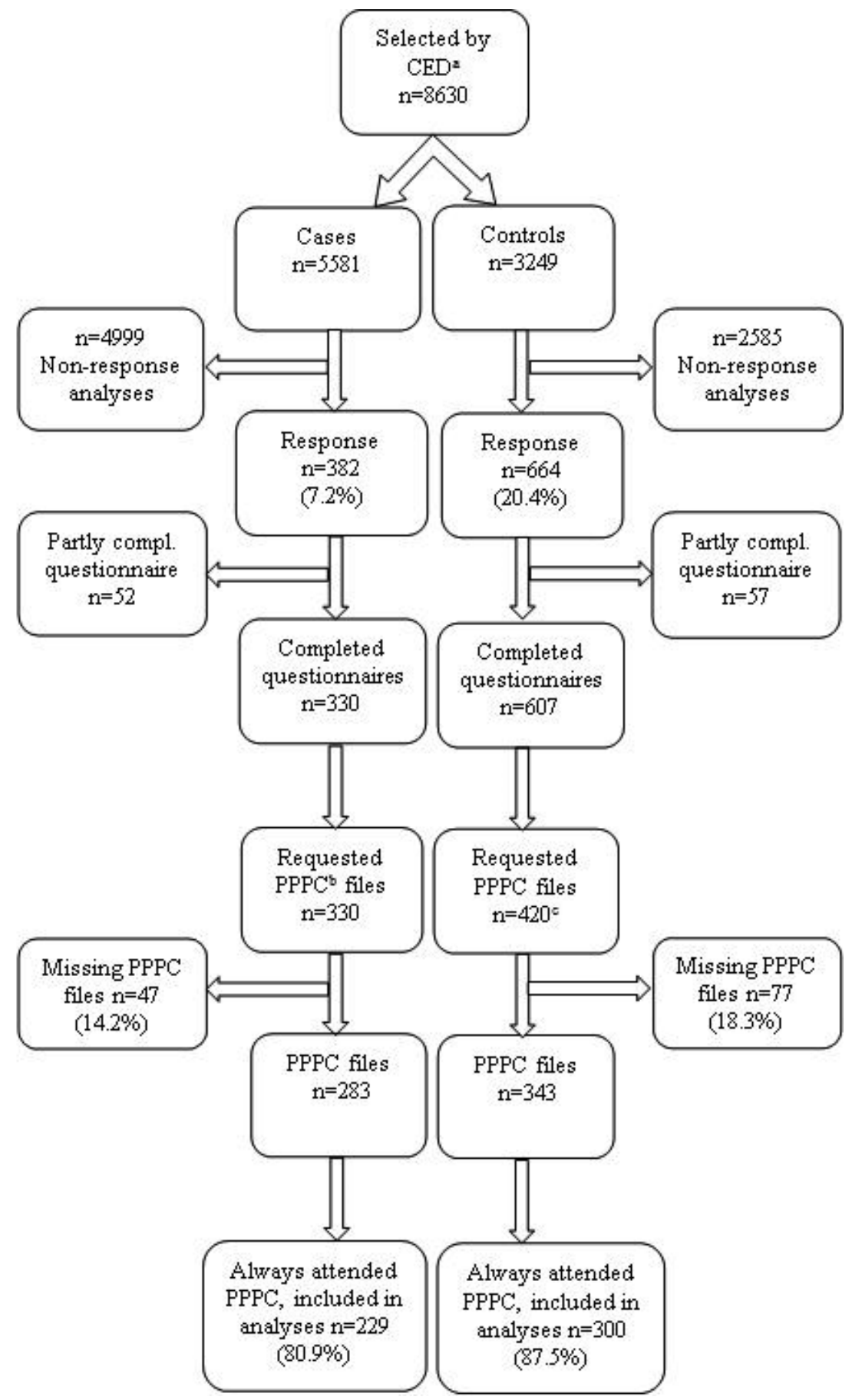

Figure 1 Inclusion flowchart

${ }^{a}$ CED: compulsory educational department, ${ }^{b}$ PPPC $=$ Preventive Pediatric Primary Care, ${ }^{c}$ randomly selected. 


\section{Ethics Statement}

Approval for conducting this study was granted by the Medical Ethics Committee of Maastricht University (METC 11-4-099, decision 22-08-2011). More details can be found elsewhere (18).

\section{Measures}

We used the standardized information from the PPPC files collected by the PPPC professionals, obstetricians or midwifes from birth onwards (21). In addition, we used information from the questionnaires such as parental education and personality traits. All 71 potential relevant factors were selected in accordance with the literature and expert knowledge. In line with the routine health examinations in PPPC and the structure of the Dutch school system, we divided the variables according to four time periods (infancy 0-4 years, early childhood 4-8 years, middle childhood 8-12 years and adolescence 12-16 years) (22). In every time period, the PPPC offers one or more non-mandatory health examinations. If the PPPC was attended at least once in every time period, and in the 0-4 time period at least twice (in the first year and at toddler age), we defined it as 'always attended' the PPPC.

\section{School dropout}

As described above, school dropouts (cases) were defined as 18 to 23 year olds who had dropped out of school during the 2010-2011 school year without attaining a basic qualification. Controls still attended school or had graduated and achieved at least a basic qualification during or at the end of the 2010-2011 school year.

\section{Bio-psychosocial determinants}

All determinants are enlisted in Table 3-6. Variables from the questionnaire are presented in italics.

The determinants in Table 3-6 are organized for each time period separately according to five domains: "health (physical and mental)", "development", "behavior, personality and lifestyle", "participation" and "environment". The time period 0-4 year additionally lists background sociodemographic variables and prenatal and perinatal variables (Table 3).

\section{Sociodemographic variables}

The respondents' own country of birth and that of their parents was used to categorize the young adults into Western (born in the Netherlands or originating from a country in Europe (not including Turkey), North America, Oceania, Indonesia or Japan) and non- 
Western ethnicity (originating from a country in Africa, South America, Asia or Turkey (not including Indonesia and Japan) (23). Family composition was categorized as living with both parents or living in a one-parent family at the time that they attended primary school. Parental education is defined as the highest education completed, classified for both parents separately into low (no education, primary education, lower vocational education or pre-vocational theoretical program), intermediate (secondary vocational education, higher general secondary education or pre-university education) and high (higher professional education or university education) (24). Socioeconomic background was based on the mean of the standardized scores for educational level of the father and the mother separately (each with originally eight ordinal categories) and four questions on material and social deprivation when at a primary school and at secondary school (each with four categories). The resulting variable was categorized into thirds using tertiles (25).

\section{Prenatal and perinatal variables}

Complications during pregnancy (hypertension, (pre)eclampsia, toxemia, HELLP syndrome or diabetes) and smoking, alcohol use, drug use or use of medication in pregnancy were dichotomized as "yes" if they were present and "no" or missing if they were not present or unknown. The Apgar score (range 1 tot 10) was measured after 1 and after 5 minutes. Preterm (gestational age of less than 37 weeks), microcephaly (head circumference less than the 3 percentile) and small for gestational age, defined as having a birth weight below -2 standard deviation scores (26) were dichotomized in "yes" in case of presence and "no" or "missing" if not present.

\section{Health}

Descriptions of physical and mental symptoms and illnesses and congenital abnormalities (with medical consequences) were categorized as "yes" in case of problems and "no" or "missing". Physical illness excluded influenza, common colds and childhood diseases, such as varicella. Learning disorders, such as dyslexia or dyscalculia, attention problems, enuresis or encopresis, hospital admissions or surgery, teenage pregnancy and having experienced an accident (with a head injury, skin burns, intoxication or fracture) were dichotomized as a "yes" in case they were present and a "no" or "missing" if they were not present or unknown. Hospital admissions or surgery was chosen as a proxy for a (diagnosed) disease and included long-term hospitalizations and major surgery, but also and especially day-care admissions such as insertion of transtympanic tubes at a middle ear effusion. An insufficient visual acuity or hearing test yes/no is determined according to the applicable guidelines (21).

\section{Development}

We used the reference data for length and weight from the 1997 Dutch Growth Study (27). Age of menarche is measured in years. Insufficient motor skills (28), language and 
speech problems and learning difficulties, including grade retention, were categorized as a "yes" in case any problems were present and as a "no" or "missing" if not present. Basic secondary education was classified into low (vocational pathways of a preparatory vocational secondary education), intermediate (theoretical learning pathway of a preparatory vocational secondary education) and high (senior general secondary education or pre-university education) (29).

\section{Behavior, lifestyle and personality}

Behavior problems were categorized in having internalizing or externalizing behavior (yes/no) according to the Child Behavior Checklist (30). Lack of motivation, eating and sleeping difficulties and smoking, alcohol use and drug use were dichotomized as a "yes" if present and as a "no" or "missing" if they were not present. Rebelliousness, defined as "wanting or feeling compelled to do something contrary to that required by some external agency" was measured with 18 items from the Social Reactivity Scale (31). The mean score was computed ( $0=$ low and $2=$ high rebelliousness) (Cronbach's $\alpha=$ 0.66). Mastery, defined as "control over life and circumstances", was measured by computing the mean of the seven items of the Pearlin and School scale $(0=10 w$ and $5=$ high mastery) (Cronbach's $\alpha=0.83$ ) (32). General self-efficacy, thinking that you are able to perform certain behaviors, was measured by computing the mean on the 16 items of Sheffer's general self-efficacy scale ( $0=10 w$ and $5=h i g h$ self-efficacy) (Cronbach's $\alpha=0.89$ ) (33). For both mastery and general self-efficacy, the respondents were asked to think of the time when they were 16. Social inadequacy was measured with computing the mean on the 15 items of the Dutch Personality Questionnaire $(0=$ low inadequacy and $2=$ high inadequacy) (Cronbach's $\alpha=0.87$ ) (34). Neuroticism and extraversion were each measured with 12 items from the Eysenck questionnaire (35). Mean scores were computed ranging from $0=$ low to 1 =high (Cronbach's $\alpha=0.84$ for both neuroticism and extraversion).

\section{Participation}

Descriptions of participation in sports (including swimming lessons), having friends (1 or none/ 2 or more) and having a part-time job outside of school were categorized as "yes" in case of non-participation or not having friends or a part-time job and "no" or "missing" otherwise. School absenteeism (yes/no) was defined as truancy or absenteeism due to sickness.

\section{Environment}

Age of parents when the respondent was born was categorized into "younger than 25 years" and "25 years or older". Birth order is defined as firstborn (1), second born (2), third born (3) etc. Family expansion (yes/no) is defined as the birth of a sibling (in every time period) or the moving in of a stepparent or a stepbrother or sister (in the time periods 4-8, 8-12 and 12-16 years). A bad relationship between parents, parental health 
problems, health problems of siblings, disturbed parent-child interaction and parenting problems were dichotomized as a "yes" in case of problems and as a "no" or "missing" otherwise. In case of suspected or identified child abuse, being bullied or being placed on a rebound facility (a facility in or outside school that provide temporary accommodation for pupils with behavioral problems) we categorized as "yes", if not as "no" or "missing". Descriptions of stressful life events were categorized as "yes" if present and "no" or "missing" if none were present. For the number of stressful life events, the events were summed.

\section{Statistical analyses}

Descriptive statistics, including frequencies, were calculated for all variables. Differences in participation of the PPPC examinations between cases and controls were determined using Chi2 tests. To examine the association between relevant demographic variables (i.e. ethnic background) and PPPC attendance (always versus not always), we conducted logistic regression analyses for each variable separately. Subsequently, we examined the association between all variables and school dropout. Only participants who attended the PPPC at least once in every time period were included into the analyses.

First, we conducted univariate logistic regression analyses for each variable separately with school dropout as the dependent variable. To cross-validate our findings, we divided the group randomly into an exploratory and a confirmatory half. Variables were selected for further analysis if the odds ratios (OR) in both halves pointed in the same direction and at least the OR in the total group was significant $(p<0.05)$ (because the power calculation was based on the total group).

Second, with the selected variables (Tables 3-6, bold variables), we conducted multivariable (stepwise) analyses beginning with the variables from the $0-4$ period. Variables were selected for further analysis if the OR in both the exploratory and the confirmatory half pointed in the same direction and at least the OR in the total group was significant $(p<0.05)$ in both the forward and backward stepwise analysis. As we aimed to identify risk factors as early in life as possible and therefore needed to avoid overadjustment, we forced the selected variables from the $0-4$ period into the next analyses in which the variables pertaining to the 4-8 period were added to a backward and forward stepwise regression analysis. Again, only if the $O R$ in both halves pointed in the same direction and the OR in the total group was significant in the forward and stepwise regression analysis, variables were taken into the next age period. Subsequently, the selected variables from the 0-8 period were forced into the next analyses and variables from the 8-12 period were added in a backward and forward stepwise regression. Then, the selected variables from the 0-12 period were forced into the next analyses and variables from the 12-16 period were added in a backward and forward stepwise regression. This resulted in a final model with variables from the 0-16 period (Table 7). 
Both the comparison of the forward and backward findings and the cross-validation in the two random halves of the sample were done to minimize capitalization on chance. We wanted to find determinants as early in life as possible and we therefore forced significant determinants in the early life phases into the models in which determinants from later life phases were tested. This simultaneously assures that determinants from later life phases are controlled for the possible confounding influence of the determinants from earlier life phases.

Finally, we conducted separate sensitivity analyses in which we looked at how ORs of the same variable differed in the results from the univariate and multivariable analyses.

\section{Results}

Figure 1 shows that there were 8630 eligible young adults, of which 5581 were dropouts and 3249 controls. Twelve percent of this group responded: 7.2 and 20.4 percent of cases and controls, respectively.

Non-participants were more likely to be men, dropouts (cases) and living in areas with cheaper houses (external zip code data (36)), and were slightly older than the participants (not tabulated). In the total group (including the eventual non-participants), men were more likely to have dropped out than women, indicating that the absence of a sex effect on dropout in the net sample is an underestimate.

As presented in Figure 1, our research sample in the current study consisted of 283 cases and 343 controls. Compared to controls, youngsters who dropped out of school more often missed health examinations from the PPPC in one or more time periods $(n=39(11.8 \%)$ and $n=50(17.9 \%)$, respectively (not tabulated)), resulting in an odds ratio of 1.62 (95\% Cl 1.03-2.55) (Table 1). Furthermore, youngsters with a non-Western background more often did not (always) attend the PPPC, as were youngster from oneparent families and youngsters living in areas with cheaper houses (OR $1.84 ; 95 \% \mathrm{Cl}$ 1.13-3.02) (not tabulated) . 
Table 1. Relevant demographic characteristics related to non-participation of the routine examinations of the Preventive Pediatric Primary Care (PPPC).

\begin{tabular}{lll}
\hline Variables & \multicolumn{2}{l}{ Missed $\geq 1$ health examinations $(\mathrm{n}=89)$} \\
\cline { 2 - 3 } & Odds ratio & $95 \%$ Confidence Interval \\
\hline School dropout & $1.62^{*}$ & $1.03-2.55$ \\
Sex; being a boy & 1.09 & $0.69-1.71$ \\
Non-Western ethnicity & $4.61^{*}$ & $2.47-8.61$ \\
One-parent family & $3.13^{*}$ & $1.83-5.37$ \\
Education mother & & \\
High education (ref) & & $0.31-1.33$ \\
Intermediate education & 0.64 & $0.68-2.44$ \\
Low education & 1.29 & \\
Education father & & $0.47-1.85$ \\
High education (ref) & & $0.51-1.73$ \\
Intermediate education & 0.96 & \\
Low educated father & 0.94 & \\
\hline
\end{tabular}

$* p<0.05$

Tables 2 to 5 show the unadjusted odds of school dropout separately for variables pertaining to the four different time periods (age groups). Having parents aged under 25 years at time of birth (OR 2.00) was related to school dropout (Table 2). Furthermore, having a non-western background (OR 2.81) or living in a one-parent family (OR 1.85) increased the odds of dropout. In the 0-4 year period suffering from a physical illness (OR 1.47) and having an insufficient hearing test (OR 1.88) were related to dropout and so were not joining a nursery or preschool (OR 2.98) and a poor relationship between parents (OR 2.76). The experience of any additional stressful life event increased the odds of dropout (OR 1.32) and the birth of a sibling in the 0-4 year period decreased the odds (OR 0.56) (Table 2). 
Table 2. Results from the univariate analyses of dropout (dependent variable) and bio-psychosocial determinants from the Preventive Pediatric Primary Care (PPPC) files and additional questionnaires in infancy (0-4 years). Only participants who always attended the PPPC are included into the analyses.

\begin{tabular}{|c|c|c|c|c|c|}
\hline \multirow{2}{*}{$\begin{array}{l}\text { Variables in the time period } \\
0-4 \text { year from the PPPC files and } \\
\text { questionnaire (italic) } \\
\text { Number of adverse findings (n) }\end{array}$} & \multirow{2}{*}{$\begin{array}{l}\text { Missing } \\
\mathrm{n}(\%)\end{array}$} & \multirow{2}{*}{$\begin{array}{l}\text { Exploratory } \\
\text { half } \\
\mathrm{n}=266 \\
\text { Odds ratio }\end{array}$} & \multirow{2}{*}{$\begin{array}{l}\text { Confirmatory } \\
\text { half } \\
n=263 \\
\text { Odds ratio }\end{array}$} & \multicolumn{2}{|l|}{ Total group } \\
\hline & & & & Odds ratio & Interval \\
\hline \multicolumn{6}{|l|}{ Sociodemographic variables } \\
\hline Sex; boys (212) & $0(0.0)$ & 1.20 & 0.91 & 1.04 & $0.73-1.48$ \\
\hline Non-western ethnicity (33) & $1(0.2)$ & $2.45^{* *}$ & $3.41^{*}$ & $2.81^{*}$ & $1.33-5.91$ \\
\hline One-parent family (69) & $0(0.0)$ & $3.48^{*}$ & 1.17 & $1.85^{*}$ & $1.11-3.08$ \\
\hline $\begin{array}{l}\text { Socioeconomic background } \\
\text { High (169) (ref) }\end{array}$ & $46(8.7)$ & & & & \\
\hline Intermediate (112) & & 1.03 & 1.09 & 1.01 & $0.63-1.63$ \\
\hline Low (202) & & 1.01 & 1.17 & 1.05 & $0.70-1.58$ \\
\hline Education father & $62(11.7)$ & & & & \\
\hline \multicolumn{6}{|l|}{ High (127) (ref) } \\
\hline Intermediate (140) & & 0.84 & 1.71 & 1.40 & $0.86-2.28$ \\
\hline Low (200) & & 1.06 & $2.17^{*}$ & 1.11 & $0.71-1.74$ \\
\hline Education mother & $54(10.2)$ & & & & \\
\hline \multicolumn{6}{|l|}{ High (93) (ref) } \\
\hline Intermediate (172) & & 0.63 & 1.35 & 0.93 & $0.56-1.55$ \\
\hline Low (210) & & 0.99 & 1.42 & 1.21 & $0.74-1.97$ \\
\hline \multicolumn{6}{|l|}{ Prenatal and perinatal variables } \\
\hline \multicolumn{6}{|l|}{ Pregnancy } \\
\hline Smoking (104) & $37(7.0)$ & 1.63 & 1.34 & $1.45^{* *}$ & $0.94-2.23$ \\
\hline alcohol use (15) & $35(6.6)$ & 5.27 & 0.36 & 0.91 & $0.32-2.61$ \\
\hline drug use (1) & $35(6.6)$ & $-{ }^{d}$ & - & - & - \\
\hline medication use (191) & $4(0.8)$ & 1.05 & 0.97 & 1.01 & $0.70-1.44$ \\
\hline complications (56) & $1(0.2)$ & 0.74 & 0.68 & 0.71 & $0.40-1.25$ \\
\hline APGAR after 1 minute ${ }^{a}$ & $200(37.8)$ & 0.95 & 0.99 & 0.99 & $0.82-1.19$ \\
\hline APGAR after 5 minutes $^{a}$ & $153(28.9)$ & 0.82 & 0.78 & 0.80 & $0.57-1.12$ \\
\hline Premature birth (28) & $0(0.0)$ & 1.47 & 0.94 & 1.14 & $0.53-2.45$ \\
\hline $\operatorname{SGA}^{\mathrm{b}}(24)$ & $0(0.0)$ & 1.44 & 0.39 & 0.93 & $0.41-2.14$ \\
\hline Microcephaly (12) & $22(4.2)$ & 3.26 & 0.36 & 1.36 & $0.43-4.27$ \\
\hline \multicolumn{6}{|l|}{ Health } \\
\hline Congenital abnormality(6) & $0(0.0)$ & 0.81 & 1.43 & 1.31 & $0.26-6.57$ \\
\hline Perinatal asphyxia (18) & $3(0.6)$ & 1.70 & 0.30 & 0.66 & $0.24-1.77$ \\
\hline Physical illness (312) & $0(0.0)$ & $1.54^{* *}$ & 1.48 & $1.47^{*}$ & $1.03-2.09$ \\
\hline Somatic complaints (289) & $0(0.0)$ & 0.77 & 0.87 & 0.83 & $0.39-1.17$ \\
\hline Enuresis/encopresis (183) & $6(1.1)$ & 0.88 & 1.30 & 1.06 & $0.74-1.52$ \\
\hline
\end{tabular}




\begin{tabular}{|c|c|c|c|c|c|}
\hline $\begin{array}{l}\text { Variables in the time period } \\
0-4 \text { year from the PPPC files and } \\
\text { questionnaire (italic) }\end{array}$ & Missing & $\begin{array}{l}\text { Exploratory } \\
\text { half } \\
n=266\end{array}$ & $\begin{array}{l}\text { Confirmatory } \\
\text { half } \\
n=263\end{array}$ & Total group & 95\% Confidence \\
\hline Number of adverse findings ( $n$ ) & $\mathrm{n}(\%)$ & Odds ratio & Odds ratio & Odds ratio & Interval \\
\hline Mental illness (0) & $0(0.0)$ & - & - & - & - \\
\hline Mental complaints (2) & $0(0.0)$ & - & - & - & - \\
\hline Had an accident (34) & $0(0.0)$ & 1.59 & 0.63 & 1.04 & $0.52-2.09$ \\
\hline Hospital admission or surgery (229) & $0(0.0)$ & 1.17 & 1.41 & 1.26 & $0.88-1.82$ \\
\hline Insufficient visual acuity (94) & $2(0.4)$ & 0.76 & 1.22 & 1.02 & $0.65-1.60$ \\
\hline Insufficient hearing test (74) & $0(0.0)$ & 1.61 & $2.29 *$ & $1.88^{*}$ & $1.15-3.09$ \\
\hline \multicolumn{6}{|l|}{ Development } \\
\hline Length as an infant & $4(0.8)$ & & & & \\
\hline \multicolumn{6}{|l|}{ P3-P97 (506) (ref) } \\
\hline <P3 (9) & & 2.53 & 0.72 & 1.07 & $0.28-4.01$ \\
\hline$>$ p97 (5) & & 1.26 & 2.86 & 2.00 & $0.33-12.06$ \\
\hline diverging curve (5) & & - & 0.72 & 2.00 & $0.33-12.06$ \\
\hline Weight as an infant & $4(0.8)$ & & & & \\
\hline \multicolumn{6}{|l|}{ P3-P97 (479) (ref) } \\
\hline$<\mathrm{P3}(15)$ & & 4.96 & 1.42 & $2.81 * *$ & $0.95-8.36$ \\
\hline$>$ p97 (12) & & - & 0.47 & $4.22 *$ & $1.13-15.79$ \\
\hline diverging curve (19) & & 1.70 & 0.85 & 1.27 & $0.51-3.17$ \\
\hline Length as a toddler & $6(1.1)$ & & & & \\
\hline \multicolumn{6}{|l|}{ P3-P97 (500) (ref) } \\
\hline$<$ P3 (7) & & 3.78 & - & 0.96 & $0.21-4.35$ \\
\hline >p97 (9) & & 0.42 & - & $0.16^{* *}$ & $0.20-1.29$ \\
\hline diverging curve (7) & & 1.26 & 0.33 & 0.51 & $0.10-2.67$ \\
\hline Weight as a toddler & $15(2.8)$ & & & & \\
\hline \multicolumn{6}{|l|}{ P3-P97 (434) (ref) } \\
\hline$<P 3(35)$ & & 1.20 & 0.82 & 1.04 & $0.52-2.08$ \\
\hline >p97 (32) & & 0.94 & 1.81 & 1.39 & $0.68-2.84$ \\
\hline diverging curve (13) & & 1.76 & 0.30 & 0.87 & $0.28-2.69$ \\
\hline Insufficient motor skills (46) & $0(0.0)$ & 0.47 & 0.64 & $0.55^{* *}$ & $0.28-1.05$ \\
\hline Language and speech problem (117) & $3(0.6)$ & 1.60 & 0.77 & 1.13 & $0.75-1.7$ \\
\hline \multicolumn{6}{|l|}{ Behavior, lifestyle and personality } \\
\hline Externalizing behavior (257) & $0(0.0)$ & 1.25 & 1.05 & 1.12 & $0.80-1.58$ \\
\hline Internalizing behavior (129) & $0(0.0)$ & 1.44 & 0.88 & 1.14 & $0.77-1.70$ \\
\hline Eating difficulties (67) & $0(0.0)$ & 1.28 & 1.17 & 1.23 & $0.74-2.06$ \\
\hline Sleeping difficulties (111) & $1(0.2)$ & 1.26 & 1.08 & 1.15 & $0.76-1.76$ \\
\hline \multicolumn{6}{|l|}{ Participation } \\
\hline No nursery or preschool (16) & $0(0.0)$ & $7.75^{* *}$ & 1.80 & $2.98 *$ & $1.02-8.69$ \\
\hline
\end{tabular}


EARLY DETERMINANTS OF SCHOOL DROPOUT

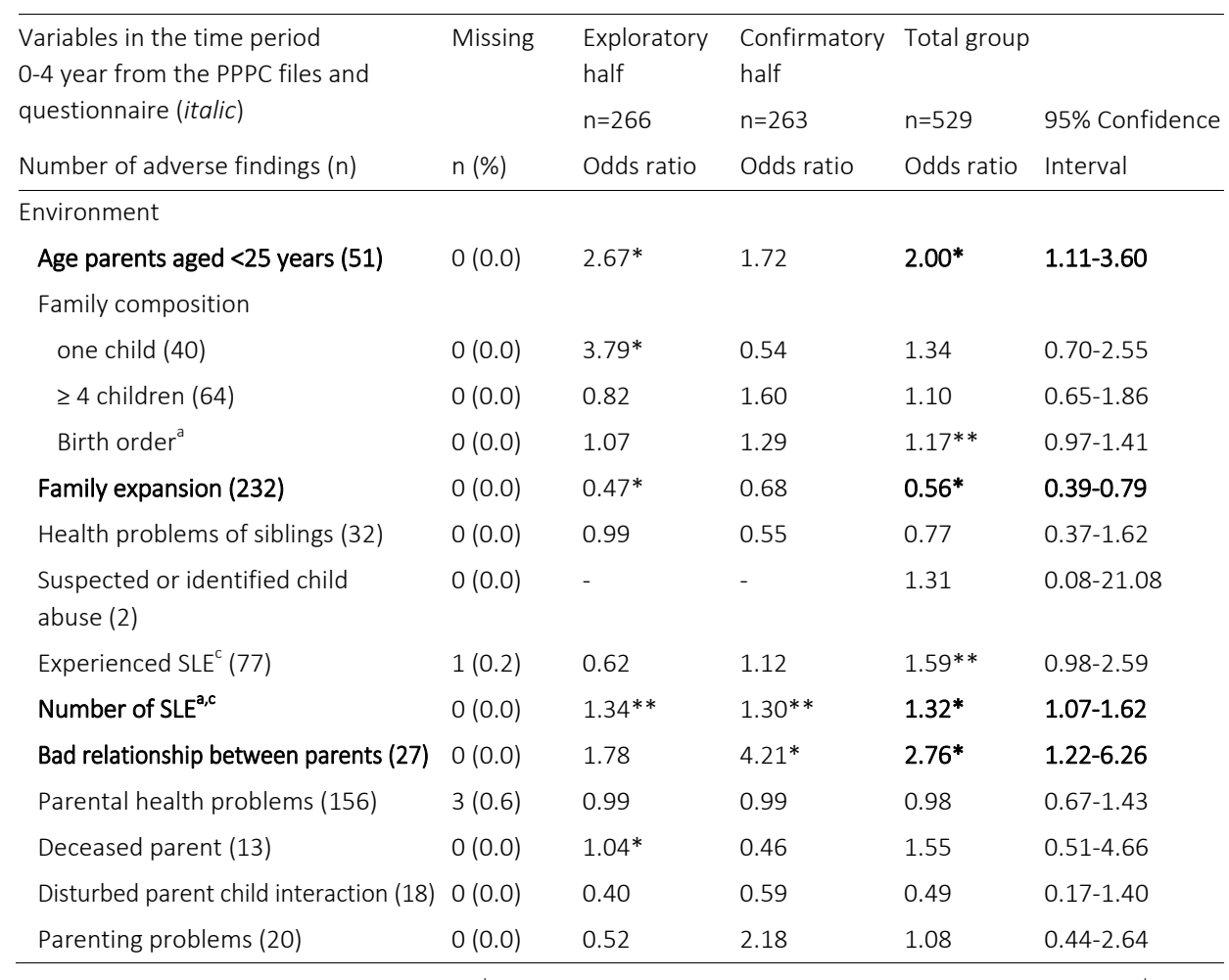

${ }^{*} \mathrm{P}<0.05,{ }^{* *} \mathrm{p}<0.10,{ }^{\mathrm{a}}$ Continuous variable, ${ }^{\mathrm{b}} \mathrm{SGA}=$ small for gestational age, ${ }^{\mathrm{c}} \mathrm{SLE}=$ stressful life events. ${ }^{\mathrm{d}}-=$ not analyzed ( $n=0$ or only cases). Variables from the questionnaire are in italic, variables included in the multivariable logistic regression analyses are in bold. 


\section{CHAPTER 3}

Table 3 shows that enuresis or encopresis (OR 1.81), learning difficulties (OR 1.97) and not attending sports were risk factor for later school dropout in the 4-8 time period (OR 3.44 ) and so was a poor relationship between parents (OR 2.30). A hospital admission or surgery in this time period decreased the odds (OR 0.33).

Table 3. Results from the univariate analyses of dropout (dependent variable) and biopsychosocial determinants from the Preventive Pediatric Primary Care (PPPC) files in early childhood (4-8 years). Only participants who always attended the PPPC are included into the analyses.

\begin{tabular}{|c|c|c|c|c|c|}
\hline \multirow{2}{*}{$\begin{array}{l}\text { Variables in the time period } \\
4-8 \text { year from the PPPC files }\end{array}$} & \multirow[t]{2}{*}{ Missing } & \multirow{2}{*}{$\begin{array}{l}\text { Exploratory } \\
\text { half } \\
n=266\end{array}$} & \multirow{2}{*}{$\begin{array}{l}\text { Confirmatory } \\
\text { half } \\
n=263\end{array}$} & \multicolumn{2}{|l|}{ Total group } \\
\hline & & & & $n=529$ & 95\% Confidence \\
\hline Number of adverse findings (n) & $\mathrm{n}(\%)$ & Odds ratio & Odds ratio & Odds ratio & Interval \\
\hline \multicolumn{6}{|l|}{ Health } \\
\hline Physical illness (140) & $1(0.2)$ & 1.23 & 0.99 & 1.09 & $0.74-1.61$ \\
\hline Somatic complaints (84) & $0(0.0)$ & 1.07 & 1.61 & 1.30 & $0.82-2.08$ \\
\hline Enuresis/encopresis (64) & $0(0.0)$ & 1.78 & $1.92^{* *}$ & $1.81^{*}$ & $1.07-3.07$ \\
\hline Mental illness (5) & $0(0.0)$ & 1.24 & $-{ }^{c}$ & 0.87 & $0.15-5.26$ \\
\hline Mental complaints (23) & $0(0.0)$ & 0.99 & $3.79 *$ & $2.11^{* *}$ & $0.90-4.95$ \\
\hline Attention problems (62) & $0(0.0)$ & 1.28 & $2.48^{*}$ & $1.69 * *$ & $0.99-2.89$ \\
\hline Learning disability (9) & $0(0.0)$ & 1.24 & 2.15 & 1.65 & $0.44-6.22$ \\
\hline Had an accident (28) & $0(0.0)$ & $2.61^{* *}$ & 0.88 & 1.55 & $0.72-3.32$ \\
\hline Hospital admission or surgery (47) & $0(0.0)$ & $0.29 *$ & $0.38 * *$ & $0.33^{*}$ & $0.16-0.67$ \\
\hline Insufficient visual acuity (114) & $0(0.0)$ & 1.30 & 1.03 & 1.18 & $0.78-1.79$ \\
\hline Insufficient hearing test (99) & $0(0.0)$ & 0.96 & 1.19 & 1.06 & $0.68-1.65$ \\
\hline \multicolumn{6}{|l|}{ Development } \\
\hline Length & $1(0.2)$ & & & & \\
\hline \multicolumn{6}{|l|}{ P3-P97 (503) (ref) } \\
\hline$<\mathrm{P} 3(10)$ & & $6.40 * *$ & 0.45 & 1.95 & $0.54-6.98$ \\
\hline$>$ p97 (11) & & 1.28 & - & 0.49 & $0.13-1.86$ \\
\hline diverging curve (4) & & - & 0.67 & 0.43 & $0.05-4.18$ \\
\hline Weight & $1(0.2)$ & & & & \\
\hline \multicolumn{6}{|l|}{ P3-P97 (432) (ref) } \\
\hline$<\mathrm{P} 3(45)$ & & 1.40 & 1.74 & 1.54 & $0.83-2.85$ \\
\hline$>$ p97 (50) & & 1.16 & 0.91 & 0.98 & $0.54-1.77$ \\
\hline diverging curve (1) & & - & - & - & - \\
\hline Insufficient motor skills (76) & $1(0.2)$ & 1.53 & 0.54 & 1.01 & $0.62-1.65$ \\
\hline Language and speech problems (112) & $1(0.2)$ & $1.87^{*}$ & 0.75 & 1.30 & $0.85-1.97$ \\
\hline Learning difficulties (62) & $0(0.0)$ & $2.08 * *$ & 1.87 & $1.97^{*}$ & $1.15-3.36$ \\
\hline \multicolumn{6}{|l|}{ Behavior, lifestyle and personality } \\
\hline Externalizing behavior (126) & $1(0.2)$ & 0.73 & 1.13 & 0.93 & $0.62-1.40$ \\
\hline Internalizing behavior (163) & $1(0.2)$ & 1.46 & 1.09 & 1.26 & $0.87-1.82$ \\
\hline
\end{tabular}




\begin{tabular}{|c|c|c|c|c|c|}
\hline \multirow{2}{*}{$\begin{array}{l}\text { Variables in the time period } \\
4-8 \text { year from the PPPC files }\end{array}$} & \multirow[t]{2}{*}{ Missing } & \multirow{2}{*}{$\begin{array}{l}\text { Exploratory } \\
\text { half } \\
n=266\end{array}$} & \multirow{2}{*}{$\begin{array}{l}\text { Confirmatory } \\
\text { half } \\
n=263\end{array}$} & \multicolumn{2}{|l|}{ Total group } \\
\hline & & & & $n=529$ & 95\% Confidence \\
\hline Number of adverse findings ( $n$ ) & $\mathrm{n}(\%)$ & Odds ratio & Odds ratio & Odds ratio & Interval \\
\hline Eating difficulties (36) & $0(0.0)$ & 0.84 & 1.95 & 1.19 & $0.60-2.34$ \\
\hline Sleeping difficulties (34) & $0(0.0)$ & 1.13 & 0.88 & 1.04 & $0.52-2.09$ \\
\hline \multicolumn{6}{|l|}{ Participation } \\
\hline Not attending sports (21) & $0(0.0)$ & $3.46^{* *}$ & $3.45^{* *}$ & $3.44^{*}$ & $1.31-9.00$ \\
\hline School absenteeism (3) & $0(0.0)$ & $2.61^{* *}$ & 0.88 & 2.63 & $0.24-29.14$ \\
\hline Not having friends (25) & $2(0.4)$ & 0.97 & 0.69 & 0.87 & $0.38-1.97$ \\
\hline \multicolumn{6}{|l|}{ Environment } \\
\hline Being bullied (17) & $3(0.6)$ & 0.62 & 1.71 & 1.17 & $0.44-3.08$ \\
\hline Family expansion (54) & $0(0.0)$ & 0.95 & 1.02 & 0.97 & $0.55-1.71$ \\
\hline Suspected or identified child abuse (8) & $0(0.0)$ & 2.50 & 2.15 & 2.21 & $0.52-9.34$ \\
\hline Experienced SLE ${ }^{\mathrm{b}}(161)$ & $0(0.0)$ & 0.71 & 1.29 & 0.94 & $0.65-1.37$ \\
\hline Number of SLE ${ }^{a, b}$ & $0(0.0)$ & 0.97 & 1.24 & 1.08 & $0.86-1.37$ \\
\hline Bad relationship between parents (32) & $0(0.0)$ & 1.59 & $3.79 *$ & $2.30^{*}$ & $1.10-4.80$ \\
\hline Parental health problems (23) & $2(0.4)$ & 2.60 & 1.18 & 1.75 & $0.75-4.06$ \\
\hline Disturbed parent child interaction (25) & $0(0.0)$ & 2.55 & 1.89 & $2.03^{* *}$ & $0.90-4.61$ \\
\hline Parenting problems (18) & $0(0.0)$ & 0.60 & 1.43 & 0.83 & $0.32-2.17$ \\
\hline
\end{tabular}

${ }^{*} \mathrm{P}<0.05,{ }^{* *} \mathrm{p}<0.10,{ }^{\mathrm{a}}$ Continuous variable, ${ }^{\mathrm{b}} \mathrm{SLE}=$ stressful life events, ${ }^{\mathrm{c}}-\mathrm{=}$ not analyzed ( $\mathrm{n}=0$ or only cases). Variables included in the multivariable logistic regression analyses are in bold.

Variables of the 8-12 time period are shown in Table 4. Family expansion (OR 3.54), somatic complaints (1.76), attention problems (OR 1.96), externalizing behavior (OR 2.85) and having an insufficient hearing test (OR 2.32) were related to dropout and so was a poor relationship between parents (OR 2.14). For girls, age of menarche played a role, as for each year between the age of 10 and 15 years the menarche delayed, the odds decreased with 0.73 . The experience of any additional stressful life event in the 8 to 12 time period increased the odds of dropout with 1.32 . 


\section{CHAPTER 3}

Table 4. Results from the univariate analyses of dropout (dependent variable) and biopsychosocial determinants from the Preventive Pediatric Primary Care (PPPC) files in middle childhood (8-12 years). Only participants who always attended the PPPC are included into the analyses.

\begin{tabular}{|c|c|c|c|c|c|}
\hline \multirow{2}{*}{$\begin{array}{l}\text { Variables in the time period } \\
8-12 \text { year from the PPPC files }\end{array}$} & \multirow[t]{2}{*}{ Missing } & \multirow{2}{*}{$\begin{array}{l}\text { Exploratory } \\
\text { half } \\
n=266\end{array}$} & \multirow{2}{*}{$\begin{array}{l}\text { Confirmatory } \\
\text { half } \\
n=263\end{array}$} & \multicolumn{2}{|l|}{ Total group } \\
\hline & & & & $n=529$ & $95 \%$ Confidence \\
\hline Number of adverse findings ( $n$ ) & $n(\%)$ & Odds ratio & Odds ratio & Odds ratio & Interval \\
\hline \multicolumn{6}{|l|}{ Health } \\
\hline Physical illness (53) & $3(0.6)$ & 1.58 & 1.02 & 1.29 & $0.73-2.28$ \\
\hline Somatic complaints (74) & $3(0.6)$ & 1.34 & 2.30 & $1.76^{*}$ & $1.07-2.89$ \\
\hline Enuresis/encopresis (20) & $4(0.8)$ & 0.49 & 1.23 & 0.87 & $0.35-2.17$ \\
\hline Mental illness (17) & $1(0.2)$ & 0.45 & 0.70 & 0.53 & $0.19-1.54$ \\
\hline Mental complaints (30) & $1(0.2)$ & $2.87 * *$ & 1.06 & 1.76 & $0.84-3.71$ \\
\hline Attention problems (43) & $6(1.1)$ & $3.15^{*}$ & 1.21 & $1.96^{*}$ & $1.04-3.69$ \\
\hline Learning disability (16) & $5(0.9)$ & 1.49 & 0.72 & 1.34 & $0.50-3.63$ \\
\hline Had an accident (14) & $2(0.4)$ & 1.56 & 2.14 & 1.76 & $0.60-5.15$ \\
\hline Hospital admission or surgery (7) & $1(0.2)$ & 3.75 & 0.70 & 1.75 & $0.39-7.92$ \\
\hline Insufficient visual acuity (131) & $5(0.9)$ & 1.33 & 1.03 & 1.19 & $0.80-1.77$ \\
\hline Insufficient hearing test (27) & $6(1.1)$ & 1.07 & $5.54^{*}$ & $2.32^{*}$ & $1.04-5.16$ \\
\hline \multicolumn{6}{|l|}{ Development } \\
\hline Length & $0(0.0)$ & & & & \\
\hline \multicolumn{6}{|l|}{ P3-P97 (504) (ref) } \\
\hline$<P 3(6)$ & & 1.88 & $c^{c}$ & 1.29 & $0.26-6.46$ \\
\hline >p97 (16) & & 1.00 & 0.23 & 0.59 & $0.20-1.71$ \\
\hline diverging curve (3) & & $-c$ & 0.68 & 0.65 & $0.06-7.16$ \\
\hline Weight & $0(0.0)$ & & & & \\
\hline \multicolumn{6}{|l|}{ P3-P97 (424) (ref) } \\
\hline$<\mathrm{P} 3(26)$ & & 0.84 & 1.14 & 0.95 & $0.43-2.11$ \\
\hline >p97 (78) & & 0.89 & 0.95 & 0.90 & $0.55-1.47$ \\
\hline diverging curve (1) & & - & - & - & - \\
\hline Menarche age ${ }^{a}$, girls only (219) & $98(30.9)$ & $0.53^{*}$ & 0.97 & $0.73^{*}$ & $0.55-0.98$ \\
\hline Insufficient motor skills (30) & $6(1.1)$ & 1.89 & 0.47 & 1.35 & $0.65-2.82$ \\
\hline Language and speech problems (36) & $7(1.3)$ & 1.26 & 0.89 & 1.05 & $0.53-2.08$ \\
\hline Learning difficulties (48) & $0(0.0)$ & 1.03 & 1.74 & 1.35 & $0.74-2.44$ \\
\hline \multicolumn{6}{|l|}{ Behavior, lifestyle and personality } \\
\hline Externalizing behavior (64) & $6(1.1)$ & $4.48^{*}$ & 1.74 & $2.85^{*}$ & $1.65-4.94$ \\
\hline Internalizing behavior (87) & $6(1.1)$ & 1.52 & 1.54 & $1.51^{* *}$ & $0.95-2.40$ \\
\hline Eating difficulties (14) & $6(1.1)$ & $4.54^{* *}$ & 0.95 & 2.42 & $0.80-7.33$ \\
\hline Sleeping difficulties (35) & $6(1.1)$ & 1.44 & $2.35^{* *}$ & $1.83^{* *}$ & $0.91-3.65$ \\
\hline
\end{tabular}




\begin{tabular}{|c|c|c|c|c|c|}
\hline \multirow{2}{*}{$\begin{array}{l}\text { Variables in the time period } \\
8-12 \text { year from the PPPC files }\end{array}$} & \multirow[t]{2}{*}{ Missing } & \multirow{2}{*}{$\begin{array}{l}\text { Exploratory } \\
\text { half } \\
n=266\end{array}$} & \multirow{2}{*}{$\begin{array}{l}\text { Confirmatory } \\
\text { half } \\
n=263\end{array}$} & \multicolumn{2}{|l|}{ Total group } \\
\hline & & & & $n=529$ & 95\% Confidence \\
\hline Number of adverse findings ( $n$ ) & $\mathrm{n}(\%)$ & Odds ratio & Odds ratio & Odds ratio & Interval \\
\hline \multicolumn{6}{|l|}{ Participation } \\
\hline Not attending sports (143) & $15(2.8)$ & 1.15 & 1.46 & 1.26 & $0.86-1.86$ \\
\hline School absenteeism (5) & $3(0.6)$ & - & - & - & - \\
\hline Not having friends (21) & $4(0.8)$ & 2.61 & 1.15 & 1.81 & $0.75-4.37$ \\
\hline \multicolumn{6}{|l|}{ Environment } \\
\hline Being bullied (45) & $6(1.1)$ & 1.76 & 1.04 & 1.27 & $0.69-2.35$ \\
\hline Family expansion (18) & $1(0.2)$ & $9.06^{*}$ & 2.17 & $3.54^{*}$ & $1.24-10.08$ \\
\hline Suspected or identified child abuse (8) & $2(0.4)$ & - & 2.14 & $3.98 * *$ & 0.80-19.92 \\
\hline Experienced SLE ${ }^{\mathrm{b}}(130)$ & $1(0.2)$ & $3.07^{*}$ & 0.92 & $1.49 * *$ & $1.00-2.21$ \\
\hline Number of SLE ${ }^{a, b}$ & $1(0.2)$ & $1.88^{*}$ & 0.91 & $1.32^{*}$ & $1.02-1.70$ \\
\hline Bad relationship between parents (53) & $2(0.4)$ & $4.70^{*}$ & 1.37 & $2.14^{*}$ & $1.20-3.83$ \\
\hline Parental health problems (25) & $497(94.0)$ & 1.67 & 1.25 & 1.13 & $0.21-6.14$ \\
\hline Disturbed parent child interaction (18) & $7(1.3)$ & 0.20 & 2.61 & 1.07 & $0.41-2.75$ \\
\hline Parenting problems (10) & $6(1.1)$ & 0.82 & 0.95 & 0.87 & $0.24-3.13$ \\
\hline
\end{tabular}

${ }^{*} \mathrm{P}<0.05,{ }^{* *} \mathrm{p}<0.10,{ }^{a}$ Continuous variable, ${ }^{\mathrm{b}} \mathrm{SLE}=$ stressful life events, ${ }^{c}$ - = not analyzed ( $\mathrm{n}=0$ or only cases or controls). Variables included in the multivariable logistic regression analyses are in bold.

Table 5 shows that in the 12-16 year period, variables significantly related to dropout are spread across all domains with the emphasis on behavior, lifestyle and personality (smoking (OR 4.77), drug use (OR 6.13), neuroticism (OR 2.97), extraversion (OR 0.49), social inadequacy (OR 1.63), self-efficacy (OR 0.47), mastery (OR 0.58) and rebelliousness (OR 2.45)). Adolescents aged 12 to 16 years, had higher odds of dropout when they were placed on a rebound facility (OR 2.90) or when their relation with their parents was disturbed (OR 2.29). However, a poor relationship between their parents also increased the odds (OR 2.07). Furthermore mental complaints, learning difficulties, not attending sports and school absenteeism were related to school dropout (OR 2.32, OR 2.32, OR 1.69 and OR 7.36 respectively). The experience of stressful life events increased the odds of dropout (OR 1.56). Moreover, the experience of any additional stressful life event increased the odds with 1.43. Teenage pregnancy occurred in five girls who always attended the PPPC, but an additional 11 girls reported a teenage pregnancy in the questionnaire (not tabulated). These were all cases. 


\section{CHAPTER 3}

Table 5. Results from the univariate analyses of dropout (dependent variable) and biopsychosocial determinants from the Preventive Pediatric Primary Care (PPPC) files and additional questionnaires in adolescence (12-16 years). Only participants who always attended the PPPC are included into the analyses.

\begin{tabular}{|c|c|c|c|c|c|}
\hline \multirow{3}{*}{$\begin{array}{l}\text { Variables in the time period } \\
12-16 \text { year from the PPPC files and } \\
\text { questionnaire (italic) } \\
\text { Number of adverse findings ( } \mathrm{n} \text { ) }\end{array}$} & \multirow[t]{2}{*}{ Missing } & \multirow{2}{*}{$\begin{array}{l}\text { Exploratory } \\
\text { half } \\
n=266\end{array}$} & \multirow{3}{*}{$\begin{array}{l}\text { Confirmatory } \\
\text { half } \\
\mathrm{n}=263 \\
\text { Odds ratio }\end{array}$} & \multicolumn{2}{|l|}{ Total group } \\
\hline & & & & $n=529$ & 95\% Confidence \\
\hline & n (\%) & Odds ratio & & Odds ratio & Interval \\
\hline \multicolumn{6}{|l|}{ Health } \\
\hline Physical illness (76) & $2(0.4)$ & 1.73 & 1.25 & 1.45 & $0.89-2.36$ \\
\hline Somatic complaints (265) & $2(0.4)$ & $1.59 * *$ & 0.77 & 1.09 & $0.77-1.54$ \\
\hline Mental illness (25) & $1(0.2)$ & 0.85 & 1.43 & 1.03 & $0.46-2.31$ \\
\hline Mental complaints (27) & $1(0.2)$ & 2.56 & 1.66 & $2.32 *$ & $1.04-5.16$ \\
\hline Attention problems (40) & $5(0.9)$ & $2.72 * *$ & 0.98 & 1.52 & $0.80-2.90$ \\
\hline Learning disability (25) & $0(0.0)$ & 2.05 & 1.44 & 1.71 & $0.76-3.84$ \\
\hline Had an accident (23) & $4(0.8)$ & 1.57 & 1.96 & 1.75 & $0.75-4.07$ \\
\hline Hospital admission or surgery (23) & $1(0.2)$ & 1.24 & 1.66 & 1.45 & $0.63-3.34$ \\
\hline Teenage pregnancy (5) & $46(8.7)$ & $-^{c}$ & - & - & - \\
\hline \multicolumn{6}{|l|}{ Development } \\
\hline Length & $3(0.6)$ & & & & \\
\hline \multicolumn{6}{|l|}{ P3-P97 (503) (ref) } \\
\hline$<P 3(5)$ & & 1.26 & 0.69 & 0.87 & $0.14-5.26$ \\
\hline >p97 (14) & & 0.94 & 0.55 & 0.73 & $0.24-2.20$ \\
\hline diverging curve (4) & & - & 0.69 & 1.31 & $0.18-9.36$ \\
\hline Weight & $3(0.6)$ & & & & \\
\hline \multicolumn{6}{|l|}{ P3-P97 (418) (ref) } \\
\hline$<P 3(32)$ & & 1.33 & 0.80 & 1.07 & $0.52-2.21$ \\
\hline >p97 (75) & & 1.41 & 1.18 & 1.27 & $0.78-2.08$ \\
\hline diverging curve (1) & & - & - & - & - \\
\hline Insufficient motor skills (5) & $3(0.6)$ & 3.82 & - & 1.99 & $0.33-12.00$ \\
\hline Language and speech problems (10) & $2(0.4)$ & $6.42 * *$ & 0.47 & 1.99 & $0.56-7.15$ \\
\hline Basic secondary education & $46(8.7)$ & & & & \\
\hline \multicolumn{6}{|l|}{ High (116) (ref) } \\
\hline Intermediate (225) & & 1.38 & 1.09 & 1.26 & $0.80-1.99$ \\
\hline Low (142) & & 1.42 & 1.85 & $1.62 * *$ & $0.99-2.67$ \\
\hline Learning difficulties (50) & $0(0.0)$ & $2.70 *$ & $2.07 * *$ & $2.32^{*}$ & $1.27-4.22$ \\
\hline \multicolumn{6}{|l|}{ Behavior, lifestyle and personality } \\
\hline Externalizing behavior (109) & $1(0.2)$ & 0.90 & $2.39 *$ & $1.50^{* *}$ & $0.98-2.29$ \\
\hline Internalizing behavior (154) & $1(0.2)$ & 1.16 & 1.04 & 1.09 & $0.74-1.58$ \\
\hline Lack of motivation (19) & $20(3.8)$ & 0.90 & $4.76^{*}$ & $2.34 * *$ & $0.91-6.06$ \\
\hline Eating difficulties (43) & $2(0.4)$ & $5.35^{*}$ & 0.50 & $2.14^{*}$ & $1.13-4.04$ \\
\hline Sleeping difficulties (94) & $2(0.4)$ & 1.29 & 1.54 & $1.47^{* *}$ & $0.94-2.29$ \\
\hline
\end{tabular}




\begin{tabular}{|c|c|c|c|c|c|}
\hline \multirow{2}{*}{$\begin{array}{l}\text { Variables in the time period } \\
12-16 \text { year from the PPPC files and } \\
\text { questionnaire (italic) } \\
\text { Number of adverse findings ( } n \text { ) }\end{array}$} & \multirow{2}{*}{$\begin{array}{l}\text { Missing } \\
\mathrm{n}(\%)\end{array}$} & \multirow{2}{*}{$\begin{array}{l}\text { Exploratory } \\
\text { half } \\
n=266 \\
\text { Odds ratio }\end{array}$} & \multirow{2}{*}{$\begin{array}{l}\text { Confirmatory } \\
\text { half } \\
n=263 \\
\text { Odds ratio }\end{array}$} & \multicolumn{2}{|l|}{ Total group } \\
\hline & & & & Odds ratio & Interval \\
\hline Smoking (22) & $12(2.3)$ & $5.89 *$ & $4.08^{*}$ & $4.77^{*}$ & $1.73-13.14$ \\
\hline Alcohol use (197) & $18(3.4)$ & 1.47 & 1.31 & $1.36 * *$ & $0.95-1.95$ \\
\hline Drug use (11) & $17(3.2)$ & 4.96 & $7.63^{* *}$ & $6.13^{*}$ & $1.31-28.68$ \\
\hline Neuroticism $^{\mathrm{a}}$ & $0(0.0)$ & 1.46 & 6.31 & $2.97^{*}$ & $1.51-5.82$ \\
\hline Extraversion $^{a}$ & $0(0.0)$ & 0.68 & $0.32 *$ & $0.49^{*}$ & $0.25-0.96$ \\
\hline Social-inadequacy ${ }^{\mathrm{a}}$ & $0(0.0)$ & 1.41 & $2.01^{*}$ & $1.63^{*}$ & $1.13-2.34$ \\
\hline Self-efficacy ${ }^{a}$ & $0(0.0)$ & $0.61^{*}$ & $0.35^{*}$ & $0.47^{*}$ & $0.35-0.65$ \\
\hline Mastery ${ }^{\mathrm{a}}$ & $0(0.0)$ & $0.56^{*}$ & $0.60^{*}$ & $0.58^{*}$ & $0.44-0.75$ \\
\hline Rebelliousness $^{\mathrm{a}}$ & $46(8.7)$ & $2.52 *$ & $2.46^{* *}$ & $2.45^{*}$ & $1.20-4.99$ \\
\hline \multicolumn{6}{|l|}{ Participation } \\
\hline Not attending sports (122) & $7(1.3)$ & $1.88^{*}$ & 1.53 & $1.69^{*}$ & $1.13-2.55$ \\
\hline School absenteeism (36) & $1(0.2)$ & $19.33^{*}$ & $5.13^{*}$ & $7.36^{*}$ & $3.01-18.01$ \\
\hline Not having friends (44) & $1(0.2)$ & 0.62 & $0.38 * *$ & $0.52 * *$ & $0.27-1.02$ \\
\hline Part-time employment (29) & $1(0.2)$ & $3.68^{*}$ & 1.07 & $1.92 * *$ & $0.90-4.10$ \\
\hline \multicolumn{6}{|l|}{ Environment } \\
\hline Being bullied (72) & $1(0.2)$ & $2.16^{*}$ & 0.64 & 1.28 & $0.78-2.10$ \\
\hline Family expansion (16) & $11(2.1)$ & $6.37^{* *}$ & 1.45 & 2.24 & $0.80-6.27$ \\
\hline Suspected or identified child abuse (8) & $1(0.2)$ & 1.23 & $7.36^{* *}$ & $4.00 * *$ & $0.80-19.98$ \\
\hline Experienced SLE ${ }^{b}(142)$ & $1(0.2)$ & $2.07 *$ & 1.26 & $1.56^{*}$ & $1.06-2.30$ \\
\hline Number of SLE ${ }^{a, b}$ & $1(0.2)$ & $1.99 *$ & 1.22 & $1.43^{*}$ & $1.16-1.76$ \\
\hline Bad relationship between parents (73) & $1(0.2)$ & $3.70 *$ & 1.52 & $2.07^{*}$ & $1.25-3.43$ \\
\hline Parental health problems (24) & $498(94.1)$ & - & 0.75 & 2.96 & $0.48-18.34$ \\
\hline Disturbed parent child interaction (32) & $3(0.6)$ & 3.01 & 1.77 & $2.29 *$ & $1.10-4.79$ \\
\hline Parenting problems (19) & $6(1.1)$ & 1.60 & 0.94 & 1.49 & $0.60-3.74$ \\
\hline Placed on a rebound facility (29) & $46(8.7)$ & $3.40 *$ & 2.52 & $2.90^{*}$ & $1.29-6.51$ \\
\hline
\end{tabular}

${ }^{*} \mathrm{P}<0.05,{ }^{*} \mathrm{p}<0.10,{ }^{\mathrm{a}}$ Continuous variable, ${ }^{\mathrm{b}} \mathrm{SLE}=$ stressful life events, ${ }^{\mathrm{c}}-=$ not analyzed ( $\mathrm{n}=0$ or only cases). Variables from the questionnaire are in italic, variables included in the multivariable logistic regression analyses are in bold.

The significant variables (in bold in Tables 2 to 5 ) were subsequently introduced in the stepwise regression analyses. Remaining variables from an earlier period were forced (not allowed to be excluded from the model) into the next stepwise model with candidate variables from later periods. Table 6 presents the final model of these age-attentive stepwise regression analyses. Having a non-Western background (OR 2.54, 95\% Cl 1.105.89) and an insufficient hearing test in the 0-4 time period (OR 1.75, 95\% Cl 0.98-3.10) increased the odds of dropout. The birth of a sibling was protective in this age period (OR 0.63, 95\% Cl 0.43-0.93). Learning difficulties (OR 1.74, 95\% Cl 0.95-3.18) and not attend- 
ing sports (OR 2.88, 95\% Cl 0.99-8.42) were risk factors in the 4-8 time period, whereas hospitalization or surgery (OR $0.2995 \% \mathrm{Cl} \mathrm{0.13-0.63)}$ in this period decreased the odds of dropout. In the 8-12 time period, externalizing behavior (OR 2.81 95\% Cl 1.53-5.14) was a risk factor and so was school absenteeism in the 12-16 year period (OR 5.62, 95\% Cl 2.18-14.52). Higher self-efficacy (scale 0-5) decreased the odds of dropout with 0.53 for every one unit increase on the self-efficacy scale (OR 0.53, 95\% Cl 0.38-0.74).

Table 6. Determinants in infancy, early and middle childhood, and adolescence predicting school drop-out in young adulthood (18-23 years). The final model

\begin{tabular}{|c|c|c|}
\hline \multirow{2}{*}{$\begin{array}{l}\text { Variables from the PPPC files and } \\
\text { questionnaires (italic) } n=529\end{array}$} & \multicolumn{2}{|r|}{ 95\% Confidence } \\
\hline & Odds ratio & Interval \\
\hline \multicolumn{3}{|l|}{ Age $0-4$ years } \\
\hline \multicolumn{3}{|l|}{ Sociodemographic } \\
\hline Non-Western ethnicity & $2.54^{*}$ & $1.10-5.89$ \\
\hline \multicolumn{3}{|l|}{ Health } \\
\hline Insufficient hearing test $0-4$ & $1.75^{* *}$ & $0.98-3.10$ \\
\hline \multicolumn{3}{|l|}{ Environment } \\
\hline Family expansion 0-4 & $0.63 *$ & $0.43-0.93$ \\
\hline \multicolumn{3}{|l|}{ Age $4-8$ years } \\
\hline \multicolumn{3}{|l|}{ Health } \\
\hline Hospitalization or surgery 4-8 & $0.29 *$ & $0.13-0.63$ \\
\hline \multicolumn{3}{|l|}{ Development } \\
\hline Learning difficulties 4-8 & $1.74^{* *}$ & $0.95-3.18$ \\
\hline \multicolumn{3}{|l|}{ Participation } \\
\hline Not attending sports $4-8$ & $2.88^{* *}$ & $0.99-8.42$ \\
\hline \multicolumn{3}{|l|}{ Age $8-12$ years } \\
\hline \multicolumn{3}{|l|}{ Behavior, lifestyle and personality } \\
\hline Externalizing behavior 8-12 & $2.81^{*}$ & $1.53-5.14$ \\
\hline \multicolumn{3}{|l|}{ Age $12-16$ years } \\
\hline \multicolumn{3}{|l|}{ Participation } \\
\hline School absenteeism 12-16 & $5.62 *$ & $2.18-14.52$ \\
\hline \multicolumn{3}{|l|}{ Behavior, lifestyle and personality } \\
\hline Self-efficacy ${ }^{\mathrm{b}} 12-16$ & $0.53^{*}$ & $0.38-0.74$ \\
\hline
\end{tabular}

${ }^{*} p<0.05,{ }^{* *} p<0.10,{ }^{a}$ PPPC $=$ Preventive Pediatric Primary Care, ${ }^{b}$ continuous variable

Variables from the questionnaire are in italic.

In the sensitivity analysis, we found some evidence for mediational pathways towards school dropout. The most major decreases in ORs, when controlling for a possible mediating variable (from a later life phase), occurred first for insufficient hearing at 0-4 years which was no longer significantly related to school dropout in the final model (Table 6) when controlled for learning difficulties in the 4-8 time period (not tabulated). 
Second, although non-western ethnicity was still significant in the final model, it decreased in association particularly by increasing non-attendance at sports in the 4-8 time period. Third, learning difficulties in the 4-8 time period were related to dropout partly because of increasing risks of externalizing behavior in the 8-12 time period. In another sensitivity analysis, we found no difference in associations, when socioeconomic status and sex were additionally controlled for in the final model: both socioeconomic status and sex were not significantly related to school dropout, nor was the pattern of associations of the other variables with school dropout substantially different from the pattern reported in Table 6.

\section{Discussion}

Using life course data from the PPPC files, collected in the everyday practice of the PPPC professionals and using an additional questionnaire, we identified risk factors and protective factors on the pathway to school dropout from birth onwards. Whereas most studies focus on single determinants, our multidimensional approach enabled us, in one study, to explore many candidate biopsychosocial determinants that might be related to school dropout in young adulthood. An age-attentive life-course approach in the modeling enabled us to identify these factors early in life and, hence, to provide tools for future interventions aimed at reducing school dropout at an early stage. Our findings supported the relevance (for school dropout) of having a non-Western background, an insufficient hearing test and the birth of a sibling (protective) in the 0-4 age period, learning difficulties, not attending sports and hospitalization or having surgery (protective) in the 4-8 age period, externalizing behavior in the 8-12 age period and school absenteeism and self-efficacy (protective) in the 12-16 age period.

Some of these determinants are not or less amenable to preventive interventions than others. However, awareness of how these determinants increase the risk of later dropout can contribute to preventive interventions for children with heightened odds of school dropout. Furthermore, the biopsychosocial domains and determinants cumulate and interact over time. Socio demographic determinants like having a non-Western ethnicity is a proxy for culturally different practices or behaviors or circumstances which we have not separately measured, factors which may be more or less amenable for prevention. Besides, our findings in the sensitivity analysis that not attending sports at the age of 4-8 years decreased the odds of school dropout for having a non-Western ethnicity suggest a pathway to school dropout through non-participation. Additionally, children with a non-Western ethnicity more often missed one or more health examinations of the PPPC. Our finding that not participating in sports at the age of 4-8 years increases the odds of school dropout is in line with other findings that participation in organized leisure activities in childhood is associated with lower dropout rates (37). A better response to no-show of non-Western families at the PPPC and stimulating their 
participation in society including sports may thus contribute to lower dropout rates. Truancy, particularly when it is persistent or frequent, is a well-known risk for school dropout (8). Furthermore, children who are frequently or persistently absent from school due to illness also tend to perform poorly in school and might therefore be at higher risk of school dropout (38). In accordance with other studies, we found academic problems to be a risk factor for dropout even in the first grades of primary school (4-8 age period) (8,39). The OR of learning difficulties in the $4-8$ time period (OR $1.97 ; 95 \% \mathrm{Cl}$ 1.15-3.36) decreased to an OR of 1.83 (95\% Cl 1.06-3.16), when externalizing behavior in the 8-12 time period was added. This suggests a pathway to school dropout through externalizing behavior. Children with early externalizing problems are more likely to become rejected and socially excluded in adolescence which may heighten their risks of dropout $(40,38)$. According to other studies, we found externalizing behavior in the age of 8 to 12 years associated to later school dropout $(3,8)$.

Youngsters' less likely dropping out of school when they had got a sibling in infancy is in line with studies reporting that children without brothers or sisters are at higher risk of dropping out (41). Possibly, the birth of a sibling provides a larger social network and practice to function within a group. Furthermore, parental attention has to be shared which increases self-dependence, but can also give competition which makes children more defensible. In our study, self-efficacy, which stimulates the student's drive and thus school attendance (40), is associated with less school dropout (11). This suggests that increasing self-efficacy at an early age may help to prevent school dropout. Children with hearing problems at a young age (0-4 year) have higher odds of school dropout, however additional learning difficulties in the 4 to 8 time period or externalizing behavior at the age of 8-12 year decreased the odds of dropout for hearing problems, suggesting that the pathway to dropout for hearing problems in the 0-4 time period leads through learning problems at the age of 4-8 year and externalizing behavior at the age of 8-12 year. We further found more youngsters having surgery or being hospitalized at the age of 4-8 years in the control group. Most surgeries concerned day admissions for hearing loss due to middle ear effusion, which is usually detected at the PPPC health examinations. Dropouts more often missed one or more health examinations; it is therefore possible that hearing problems remained undetected and caused learning or behavior problems. Furthermore, parents of the controls may be more closely watching the health status of their children or perhaps being in the hospital in early childhood brings positive attention from teachers and peers in primary school. Since we are not sure of the explanation, further research is required. Although we did not include menarche age and teenage pregnancy in the multivariable regression analyses, because they are only relevant in girls, we should consider it risk factors for school dropout, in line with other studies $(1,42)$. Girls with an early menarche have higher rates of externalizing problems and school problems $(43,44)$, which may explain our finding that a younger age at menarche is a risk factor for school dropout. Besides, early puberty is associated with teenage pregnancy, which in turn is a risk for dropout $(45,46)$. 
School dropout reduces the chance of finding a job (47) and low socioeconomic status is associated with poorer health (48). Furthermore, parental socioeconomic status is a strong predictor of a child's academic achievement (15). Since school dropout is also a public health problem, PPPC might play a role in breaking this negative spiral by monitoring children with an increased dropout risk from birth onwards and empower their strengths. However one-parent families and families with a non-Western background, who often have a lower income, participate less in the health examinations of the PPPC and so do families living in areas with cheaper houses. Therefore, the effect of socioeconomic status in our study might be underestimated. Moreover, non-participation in the PPPC might increase the socioeconomic health differences and inequalities. Especially families suffering from multiple and complex problems are a hard-to-reach group (49). To reach these families, an active approach is required in addition to a more easily accessible and approachable PPPC (50).

We used candidate determinants from the whole life-course and, acknowledging the biopsychosocial context, from various domains. Many determinants are collected in PPPC practice and interventions may, we hope, easily build upon this practice. Furthermore, our design and approach was highly systematic in trying to exclude selection bias and capitalization on chance and trying to particularly appreciate the influence of determinants from earlier life. Falsely removing the statistically significant early life determinants -due to overadjustment as a result of controlling for mediators- did not occur because these early phase determinants were forced into the model with candidate determinants from later phases (where mediational processes could take place). We admit that overadjustment could still have resulted in underestimated odds ratios in the multivariable analyses, but this was evaluated further as possible mediational processes in the sensitivity analyses, where the most major processes were described. The forced entry of the initially significant early life phase determinants also controls for their potential confounding influence on the associations of later life phase determinants and school dropout.

Our approach may still have led to an oversimplified representation of the pathways towards school dropout. Given the abundance of candidate determinants and the resulting difficulty of specifying direct acyclic graphs (DAGs) $(51,52)$ and the many specified pathways and mechanisms in such graphs, we could, however, not think of other more insightful statistical approaches of the inter-individual variation in pathways towards school dropout. Although our model is thus also too simple to exclude unmeasured mediator-outcome confounding, interactions between variables, and intermediate confounding (53), we still think it provides robust quantitative evidence on the major early life determinants of later school dropout. However, to get further insight into and understanding of the complex pathways, we also conducted interviews with participants and focus groups with parents, teachers and PPPC physicians that will help to further interpret and contextualize the results. These will also further explore the needs and requirements of all stakeholders to prevent school dropout. Our aim with 
both the current quantitative and the future qualitative information is to develop a personalized prediction model and ultimately an effective intervention that is able to prevent dropout at an early stage.

There are some further limitations that need to be addressed. First, this study has an exploratory character, but the systematic way of analyzing by comparing the forward and backward findings and the cross-validation in the two random halves of the sample minimizes capitalization on chance. The model certainly needs further validation in other samples, but also across various subgroups (e.g. according to sex, ethnic background, socioeconomic status etc.). Second, to avoid selection bias, the control group was highly similar to the cases at the start of the school year 2010-2011 (both were still attending school). The reported odds ratios thus only apply to an already initially disadvantaged population, as, at the age of 18 to 23 , both cases and controls already should have attained their basic qualification. Simultaneously, this might imply that we have underestimated the associations compared to a study using all possible controls and cases. Although, for future studies, we recommend a longitudinal design in which all possible cases and controls, at any age, would be included, we think that our efforts in avoiding selection bias has provided valid information on determinants of school dropout. Further selection bias may have taken place, since only available PPPC files from children who previously completed a questionnaire and who visited the PPPC in every time-period are included into the analysis. We therefore compared participants and non-participants regarding their dropout status, age, sex and zip-code level socioeconomic status (36). Since men, dropouts (cases) and youngsters living in areas with cheaper houses less often completed the questionnaire and cases, youngsters with a non-Western background, living in one-parent families or in areas with cheaper houses or having a lower educated mother more often missed one or more PPPC health examinations, the absence of a sex and socioeconomic status effect on dropout in the final sample is probably an underestimate. Third, information bias is also of relevance. Some determinants, for example child abuse, have a low prevalence in our study and may therefore not be identified as a risk factor despite its high ORs. Possibly, this is underreported in the PPPC files. Further research with a larger sample is needed. Furthermore, the data described in the PPPC files were not collected for the purpose of this study, so important information might be missing. In order to obviate this, an additional questionnaire was sent, in which we asked for personality traits thinking of the time when they were 16 years of age. However, recall bias still cannot be excluded. As the information in the PPPC files is collected from birth onwards, this information is not affected by recall bias. However, the data in the PPPC files have been compiled by many different PPPC professionals, each with their own interpretations and descriptions of certain situations, symptoms and life-course determinants. Because the PPPC professionals use this information in their daily practice, the ecological validity of our findings should, on the other hand, be high. 


\section{Conclusion}

To prevent school dropout, PPPC professionals should not wait until imminent dropout, but they should try to identify and tackle risk factors in childhood as early as possible. They can advise parents on understanding the long-term effects of hearing problems in infancy and the importance of participation in sports in early childhood, and support parents to strengthen protective factors such as giving a child positive attention and promoting self-efficacy. Furthermore, an important reduction of school dropout can also be attained by more actively approaching and helping parents and youngsters who withdraw from public health care. Further research with a larger sample is needed to validate our model across subgroups and to evaluate whether targeting the risk factors is truly effective in tackling school dropout. Future interviews and focus groups will help to further interpret and contextualize the results and to emphasize the wide individual differences in the context of school dropout.

\section{Acknowledgements}

We thank the young adults who participated in the study for providing their valuable information and permitting us to link their PPPC file to the questionnaire. We also thank the students who helped to enter the data from the PPPC files into SPSS. 


\section{References}

1. Freudenberg N, Ruglis J (2007) Reframing school dropout as a public health issue. Prev Chronic Dis 4: 1-11.

2. Smeyers $P$ (2006) The relevance of irrelevant research: The irrelevance of relevant research. In: Smeyers P, Depaepe M, editors. Educational research: Why 'what works' doesn't work. Dordrecht: : Springer. pp. 95-108.

3. Breslau J (2010) Health in Childhood and adolescence and High School Dropout. University of California, Davis.

4. Bradshaw CP, O'Brennan LM, McNeely CA (2008) Core competencies and the prevention of school failure and early school leaving. New Directions for Child and Adolescent Development 122: 19-32.

5. Jimerson S, Egeland B, Scroufe L, Carlson B (2000) A prospective longitudinal study of high school dropouts; Examining multiple predictors across development. J School Psychol 38: 525-549.

6. Herweijer L (2008) Gestruikeld voor de start. De school verlaten zonder startkwalificatie. Den Haag: Sociaal en Cultureel Planbureau.

7. Tanner-Smith E, Wilson S (2013) A Meta-analysis of the Effects of Dropout Prevention Programs on School Absenteeism. Prevention Science 14: 468-478.

8. De Witte K, Cabus S, Thyssen G, Groot W, van den Brink HM (2013) A critical review of the literature on school dropout. Educational Research Review 10: 13-28.

9. Bradshaw C, Schaeffer C, Petras H, lalongo N (2010) Predicting Negative Life Outcomes from Early Aggressive - Disruptive Behavior Trajectories: Gender Differences in Maladaptation Across Life Domains. Journal of Youth and Adolescence 39: 953-966.

10. Alatupa S, Pulkki-Råback L, Hintsanen M, Mullola S, Lipsanen J, et al. (2011) Childhood Disruptive Behaviour and School Performance across Comprehensive School: A Prospective Cohort Study. Psychology 2: 542-551.

11. Bosma H, Theunissen M-J, Verdonk P, Feron F (2014) Low control beliefs in relation to school dropout and poor health: findings from the SIODO case-control study. BMC Public Health 14: 1237.

12. Suhrcke M, de Paz Nieves C (2011) The impact of health and health behaviours on educational outcomes in high-income countries: a review of the evidence. Copenhagen: WHO Regional Office for Europe.

13. Theunissen M-J, de Man I, Verdonk P, Bosma H, Feron F (2015) Are Barbie and Ken too cool for school? A case-control study on the relation between gender and dropout. The European Journal of Public Health 25: 57-62.

14. O'Higgins N, D'Amato M, Caroleo F, Barone A (2007) Gone for Good? Determinants of school dropout in Southern Italy. Giornale degli Economisti 66: 207-245.

15. Archambault I, Janosz M, Morizot J, Pagani L (2009) Adolescent behavioral, affective and cognitive engagement in school: relationship to dropout. J Sch health 79: 408-415.

16. Kuo AA, Inkelas M, Lotstein DS, Samson KM, Schor EL, et al. (2006) Rethinking Well-Child Care in the United States: An International Comparison. Pediatrics 118: 1692-1702.

17. Brugman E, Reijneveld S, Verhulst F, Verloove-Vanhorick S (2001) Identification and management of psychosocial problems by preventive child health care. Archives of pediatrics and adolescent medicine 155: 462-469.

18. Theunissen M-J, Griensven van I, Verdonk P, Feron F, Bosma H (2012) The early identification of risk factors on the pathway to school dropout in the SIODO study: a sequential mixed-methods study. BMC Public Health 12: 1033.

19. European Council (2003) Council Conclusions of 5 May 2003 on Reference Levels of European Average Performance in Education and Training (Benchmarks). Official Journal of the European Union 46: 3-4.

20. United Nations Educational Scientific and Cultural Organization (UNESCO) ( 5 September 2011) The revised International Standard Classification of Education (ISCED 2011). Paris.

21. NCJ [Dutch centre for Preventive Child and Youth Health care] Applicable guidelines for Preventive Child and Youth Health care. Utrecht. Available : https://www.ncj.nl/programmalijn-kennis/richtlijnen/ overzicht-richtlijnen. Accessed 28 August 2014. 
22. Platform jeugdgezondheidszorg (25 juni 2003) Richtlijn Contactmomenten. Basistakenpakket jeugdgezondheidszorg 0-19 jaar. Richtlijn 1. Den Haag.

23. Statistics Netherlands (22 February 2012) Dutch population. Available : http://statline.cbs.nl/Statweb/ publication/?VW=T\&DM=SLNL\&PA=70748NED\&D1=0,2,4,16,18,20,22,24\&D2=a\&D3=0\&D4=a\&D5=1\&HD =090707-1905\&HDR=T\&STB=G4,G2,G1,G3. Accessed 2 December 2013.

24. Volksgezondheid Toekomst Verkenning Nationaal Kompas Volksgezondheid [The National Public Health Compass] (2013). 4.11 ed. Bilthoven: RIVM. Available: http://www.nationaalkompas.nl/bevolking/ scholing-en-opleiding/indeling-opleidingsniveau/. Accessed: 28 march 2013.

25. Shavers VL (2007) Measurement of socioeconomic status in health disparities research. J Natl Med Assoc 99: 1013-1023.

26. Visser GHA, Eilers PHC, Elferink-Stinkens PM, Merkus HMWM, Wit JM (2009) New Dutch reference curves for birthweight by gestational age. Early Human Development 85: 737-744.

27. Fredriks AM, van Buuren S, Burgmeijer RJF, Meulmeester JF, Beuker RJ, et al. (2000) Continuing Positive Secular Growth Change in the Netherlands 1955-1997. Pediatr Res 47: 316-323.

28. Laurent Angulo M, Brouwers-de Jong E, Bijlsma-Schlösser J, Bulk-Bunschoten A, Pauwels J, et al. (2005) Ontwikkelingsonderzoek in de jeugdgezondheidszorg. Het Van Wiechenonderzoek - De Baecke-Fassaert Motoriektest Assen: Van Gorcum

29. Department for Credential Evaluation SBB (2012) The Dutch educational system. Zoetermeer: National Reference Point Netherlands [NRP]. Available: http://www.nlnrp.nl/current-educational-system.html. Accessed: 28 August 2014.

30. Achenbach T (2009) The Achenbach System of Empirically Based Assessment (ASEBA): Development, Findings, Theory, and Applications. Burlington: VT: University of Vermont Research Center for Children, Youth and Families.

31. Klabbers G, Bosma H, van den Akker M, van Boxtel MPJ, Kempen GIJM, et al. (2009) Measuring Rebelliousness and Predicting Health Behaviour and Outcomes. Journal of Health Psychology 14: 771-779.

32. Pearlin LI, Schooler C (1978) The Structure of Coping. Journal of Health and Social Behavior 19: 2-21.

33. Sherer M, Maddux J, Mercandante B, Prentice-Dunn S, Jacobs B, et al. (1982) The self-efficacy scale: construction and validation. Psychol Rep 51: 663-671.

34. Luteijn F, Starren J, van Dijk H (2000) Handleiding bij de NPV, tweede herziene versie. [Manual for the Dutch Personality Questionnaire: revised edition]. Lisse: Swets en Zeitlinger.

35. Sanderman R, Arrindell WA, Ranchor AV, Eysenck HJ, Eysenck SBG (1995) Het meten van de persoonlijkheidskenmerken met de Eysenck Personality Questionnaire (EPQ). Een handleiding [The assessment of personality with Eysenck Personality Questionnaire (EPQ): A manual]. Groningen, The Netherlands: University of Groningen, Northern Centre for Healthcare Research.

36. Statistics Netherlands (2006) Kerncijfers postcodegebieden 2004. Voorburg. Available: http://www.cbs.nl/nl-NL/menu/themas/dossiers/nederland-regionaal/cijfers/incidenteel/maatwerk/kern cijfers-postcodegebieden-2004.htm. Accessed 20 August 203.

37. Howie LD, Lukacs SL, Pastor PN, Reuben CA, Mendola P (2010) Participation in Activities Outside of School Hours in Relation to Problem Behavior and Social Skills in Middle Childhood. Journal of School Health 80: 119-125.

38. Weitzman M, Klerman LV, Lamb G, Menary J, Alpert JJ (1982) School Absence: A Problem for the Pediatrician. Pediatrics 69: 739-746.

39. Newcomb MD, Abbott RD, Catalano RF, Hawkins JD, Battin-Pearson S, et al. (2002) Mediational and deviance theories of late high school failure: Process roles of structural strains, academic competence, and general versus specific problem behavior. Journal of Counseling Psychology 49: 172-186.

40. Dowrick P, Crespo N (2005) School Failure. In: Gullotta T, Adams G, editors. Handbook of Adolescent Behavioral Problems: Springer US. pp. 589-610.

41. Traag T, van der Velden R (2011) Early school-leaving in the Netherlands: the role of family resources, school composition and background characteristics in early school-leaving in lower secondary education. Irish Educational Studies 30: 45-62. 


\section{CHAPTER 3}

42. Uzun AK, Orhon FS, Baskan S, Ulukol B (2013) A comparison between adolescent mothers and adult mothers in terms of maternal and infant outcomes at follow-ups. Journal of Maternal-Fetal and Neonatal Medicine 26: 454-458.

43. Mendle J, Turkheimer E, Emery RE (2007) Detrimental psychological outcomes associated with early pubertal timing in adolescent girls. Developmental Review 27: 151-171.

44. Celio M, Karnik NS, Steiner H (2006) Early maturation as a risk factor for aggression and delinquency in adolescent girls: a review. International Journal of Clinical Practice 60: 1254-1262.

45. De Genna NM, Larkby C, Cornelius MD (2011) Pubertal Timing and Early Sexual Intercourse in the Offspring of Teenage Mothers. Journal of Youth and Adolescence 40: 1315-1328.

46. Deardorff J, Gonzales NA, Christopher FS, Roosa MW, Millsap RE (2005) Early Puberty and Adolescent Pregnancy: The Influence of Alcohol Use. Pediatrics 116: 1451-1456.

47. De Ridder KAA, Pape K, Cuypers K, Johnsen R, Holmen T, et al. (2013) High school dropout and long-term sickness and disability in young adulthood: a prospective propensity score stratified cohort study (the Young-HUNT study). BMC Public Health 13: 941.

48. Elgar FJ, Pförtner T-K, Moor I, De Clercq B, Stevens GWJM, et al. Socioeconomic inequalities in adolescent health 2002-2010: a time-series analysis of 34 countries participating in the Health Behaviour in Schoolaged Children study. The Lancet. 03 february 2015 ed: Elsevier.

49. Rots-de Vries C, van de Goor I, Karien S, Garretsen H (2011) Psychosocial child adjustment and family functioning in families reached with an assertive outreach intervention. Scandinavian Journal of Caring Sciences 25: 269-276.

50. Rots-de Vries C, van de Goor I, Stronks K, Garretsen H (2011) Evaluation of an assertive outreach intervention for problem families: intervention methods and early outcomes. Scandinavian Journal of Caring Sciences 25: 211-219.

51. Shrier I, Platt RW (2008) Reducing bias through directed acyclic graphs. BMC Med Res Methodol 8:70.

52. Law GR, Green R, Ellison GTH (2012) Confounding and causal path diagrams in: Tu Y-K, Greenwood DC (eds). Modern Methods for Epidemiology. London: Springer.

53. Richiardi L, Bellocco R, Zugna D (2013) Mediation analysis in epidemiology: methods, interpretation and bias. International Journal of Epidemiology 42: 1511-1519. 


\section{Chapter 4}

\section{Are Barbie and Ken too cool for school? A case-control study on the relation between gender and dropout}

Published as:

Theunissen M-J, de Man I, Verdonk P, Bosma H, Feron F. Are Barbie and Ken too cool for school? A case-control study on the relation between gender and dropout.

The European Journal of Public Health. 2015;25(1):57-62. 


\section{Abstract}

\section{Objectives}

As school dropout is an important public health problem which needs to be addressed, we set out to examine whether and how, beyond the well-known effects of sex, gender beliefs and self-reported masculinity and femininity are related to school dropout.

\section{Methods}

The study used a case-control design, consisting of 330 dropout cases and 330 controls still attending school. The respondents, aged between 18 and 23, living in the southeast of the Netherlands, were sent a self-administered questionnaire. Separate logistic regression analyses for the male and female participants were used to explore the relation between dropout and gender, controlling for sociodemographic determinants.

\section{Results}

As indicated by significant curvilinearity, young women were less likely to drop out when they occupied an intermediate positions on the gender variables. Odds of dropout were elevated among highly masculine women (OR=2.1, 95\% Cl: 1.1-4.1), and, as indicated by significant interactions, also among highly masculine men with strong normative masculine beliefs, and in feminine men who simultaneously considered themselves low on masculinity.

\section{Conclusions}

Beyond sex, gender is important in the explanation of school dropout. In order to prevent dropout, public health professionals should assess, monitor, and intervene on the basis of gender characteristics. 


\section{Introduction}

School dropout is an important public health problem (1). Poor health might be implicated in the pathways leading to dropout and dropout in turn might further compromise health (2). Dropout reduces the chances of finding a job and is also strongly linked to social exclusion. Although some countries have been able to reduce dropout rates, it is still a major problem for many countries and particularly for the individuals concerned $(1,3)$. Sex differences in school dropout are consistent across cultures: boys drop out more often. However, sex and gender are seriously underinvestigated in public health research (4). In order to contribute to the prevention of dropout, further research is needed that looks beyond the "classical" determinants of school dropout: being a boy, low socioeconomic background, belonging to an ethnic minority, and coming from a single parent family. Finding new determinants might help public health professionals to intervene before further harm occurs. We therefore set out to examine whether, beyond the influences of sex and the other classical determinants, gender is related to school dropout.

Sex generally refers to the biological differences between women and men, while gender refers to the culturally determined beliefs, behaviors and characteristics that are associated with, but not determined by sex. Gender differences are learned, may change over time, and vary significantly both within and between cultures (4-6). Masculinity and femininity are often seen as separate dimensions which allow an individual to be categorized as highly masculine, highly feminine, both or neither $(7,8)$. Femininity refers to a person's identification with stereotypical feminine gender role traits, such as compassion or sensitivity (9) and masculinity to identification with stereotypical masculine ones, such as self-reliance or ambition. Hegemonic masculinity is defined as the most widely accepted and normative beliefs about being a man in a given society. It implies a pattern of practice that allows the domination of men over women $(10,11)$. Generally masculine characteristics have become more acceptable for women and stereotypes less absolute: masculine characteristics have increased in women while maintaining feminine ones $(12,13)$. This might give women a wider variety of skills and characteristics, enabling them to better cope with different situations and life's difficulties $(5,14)$.

Gendered interactions, such as how to present and relate to peers, or beliefs about whether or not academic performance is considered masculine, may affect pupils' perceptions of school $(15,16)$. Men and women are expected to behave according to culturally prescribed behaviors and traits that dictate how males and females should act (17). Boys who hold stereotyped beliefs regarding these gender roles are more likely to engage in problem behavior (18) and might be more likely to drop out of school. Deviant behavior might earn boys the respect of their peers, prove their masculinity, and make them "too cool for school" (19-21). However, research on masculinity and femininity in the process resulting in school dropout is still scarce and limited to masculinity 
in boys and femininity in girls. Hyper-masculine boys are low on learning interests, school engagement, and high on risk-taking behavior. Similarly, hyper-feminine girls also tend to show little interest in school and career and to show antisocial attitudes and hostility towards other women (22).

Using Dutch case-control data on 660 young adults, we set out to examine the effect on school dropout of both masculinity and femininity in separate groups of male and female young adults. In order to fully explore the gender effect, we also looked at whether there is a golden mean in gender, by analyzing complex relations (in terms of curvilinearity and interactions), and studied whether the gender effect is independent of the classical determinants. The aim was to find out whether gender is a candidate characteristic for inclusion in the repertoire of public health professionals trying to reduce school dropout.

\section{Methods}

\section{Study population}

This study was part of the Dutch SIODO (Stay In Or Drop Out) study, a sequential mixedmethods study focusing on the early identification of risk factors on the pathway to school dropout (23). The current paper is based on the quantitative case-control data from SIODO. In November 2011, the municipal compulsory education department of the city of Eindhoven (The Netherlands), selected all young adults aged 18 to 23 years who had not yet met the Dutch minimum educational requirement (which is equivalent to higher general secondary education, pre-university education, or intermediate vocational education, level 2) at the start of the 2010 - 2011 school year (24, 25). At this age, they normally should have obtained the required "starting qualification". A proportion of these young adults remained in school during the 2010-2011 school year in order to obtain this qualification (the controls), while others dropped out of school during that year without qualification (the cases). Cases and controls being so similar at the start of the 2010-2011 school year was supposed to avoid selection bias. We sent a self-administered questionnaire with an informed consent form to the selected participants. A power analysis indicated that 290 cases and 290 controls should be sufficient; we eventually were able to include 330 cases and 330 controls. Approval for conducting this study was granted by the Medical Ethics Committee of Maastricht University (METC 11-4-099, decision 22-08-2011). More details can be found elsewhere (23). 


\section{Measures}

\section{Gender variables}

The Adolescent Masculinity Ideology in Relationships Scale (AMIRS) (26) is composed of 12 belief statements about appropriate behavior for males within interpersonal relationships among peers (e.g. "Guys should not let it show when their feelings are hurt"). The range for each item is 1 (completely disagree) to 4 (completely agree). Negatively worded items receive a reversed score. Composite scores are calculated by taking the mean across items when 7 or more items have been completed. Higher scores reflect greater alignment with norms of hegemonic masculinity within the context of interpersonal relationships. Internal consistency as measured by Cronbach's $\alpha$ was 0.83 (reported Cronbach's a 0.67-0.71).

The Inauthentic Self in Relationship (ISR) subscale from the Adolescent Femininity Ideology Scale (AFIS) reflects the internalizing conventional femininity ideologies of inauthenticity in relationships (e.g. "I worry that I make others feel bad if I am successful") (27). The ISR is a 10-item scale using a five-point Likert scale (1=completely disagree, $5=$ completely agree). Composite scores are calculated by taking the mean across items when 6 or more items have been completed. Higher scores indicate more inauthenticity. Internal consistency as measured by Cronbach's a was 0.70 (reported Cronbach's a 0.67-0.81).

We also asked the respondents "how feminine/masculine do you think you were at the age of 16", exemplified by "helpful, sensitive, shy, caring, understanding, friendly" as feminine traits and "acting as a leader, ambitious, dominant, independent, aggressive, tough behavior" as masculine traits (7), asking them to rate their femininity/masculinity on a 1-10 scale. A higher score for the separate femininity and masculinity items indicates assigning oneself more feminine or masculine traits, respectively. Both indicators are valid indicators of gender identity $(12,28,29)$.

\section{Covariates}

Age was assessed on August 1, 2010. The respondents' own country of birth and that of their parents was used to categorize the young adults into Western (born in the Netherlands or originating from a country in Europe (not including Turkey), North America, Oceania, Indonesia or Japan) and non-Western ethnicity (originating from a country in Africa, South America, Asia or Turkey (not including Indonesia and Japan) (30). We asked participants about their family composition at the time when they attended primary school. The answers were categorized as living with both parents or living in a one-parent family. The highest education completed was classified, for both parents separately, into low (no education, primary education, lower vocational education or pre-vocational theoretical program), intermediate (secondary vocational education, higher general secondary education or pre-university education) and high (higher professional education or university education) $(31,32)$. 


\section{Statistical analyses}

Descriptive statistics, including frequencies, means and standard deviations (SD), were calculated for all variables. Differences in gender variables between male and female participants and between cases and controls were determined using chi-square tests for categorical variables and t-tests and one-way between-group analysis of variance for continuous variables. To investigate the association between gender and dropout, we performed logistic regression analyses with dropout as dependent variable and selfreported femininity, self-reported masculinity, mean AMIRS scores and mean AFIS scores as independent variables. Age, ethnicity, family composition and parental education were controlled for. Separate analyses were conducted for men and women. In order to improve the power of our study, we initially conducted the analyses with the continuous gender variables, estimating odds ratios of quadratic terms (curvilinearity) and product terms between gender variables (interactions). In the case of a curvilinear relationship, the gender variables were categorized into tertiles to examine the shape of the curve. In the case of interactions, the (continuous) effect of one gender variable was estimated separately for the groups with high (mean + 1 SD) and low (mean - 1 SD) scores on the other gender variable. All analyses were performed using SPSS 20.0.

\section{Results}

Figure 1 shows that there were 8,630 eligible young adults, 5,381 of whom were dropouts and 3,249 controls. Twelve percent of this group responded: 7.2 and 20.4 percent of cases and controls, respectively. Table 1 shows that, compared to the control group, the case group comprised more participants of non-Western ethnicity both male (14.0 vs. 6.2 percent) and female (15.0 vs. 4.5 percent), and were on average 0.5 years older. Female participants who dropped out of school were more likely to have lived in oneparent families when in primary school (25.6 vs. 8.0 percent), considered themselves less feminine and scored higher on the AMIRS compared to female participants in the control group. 


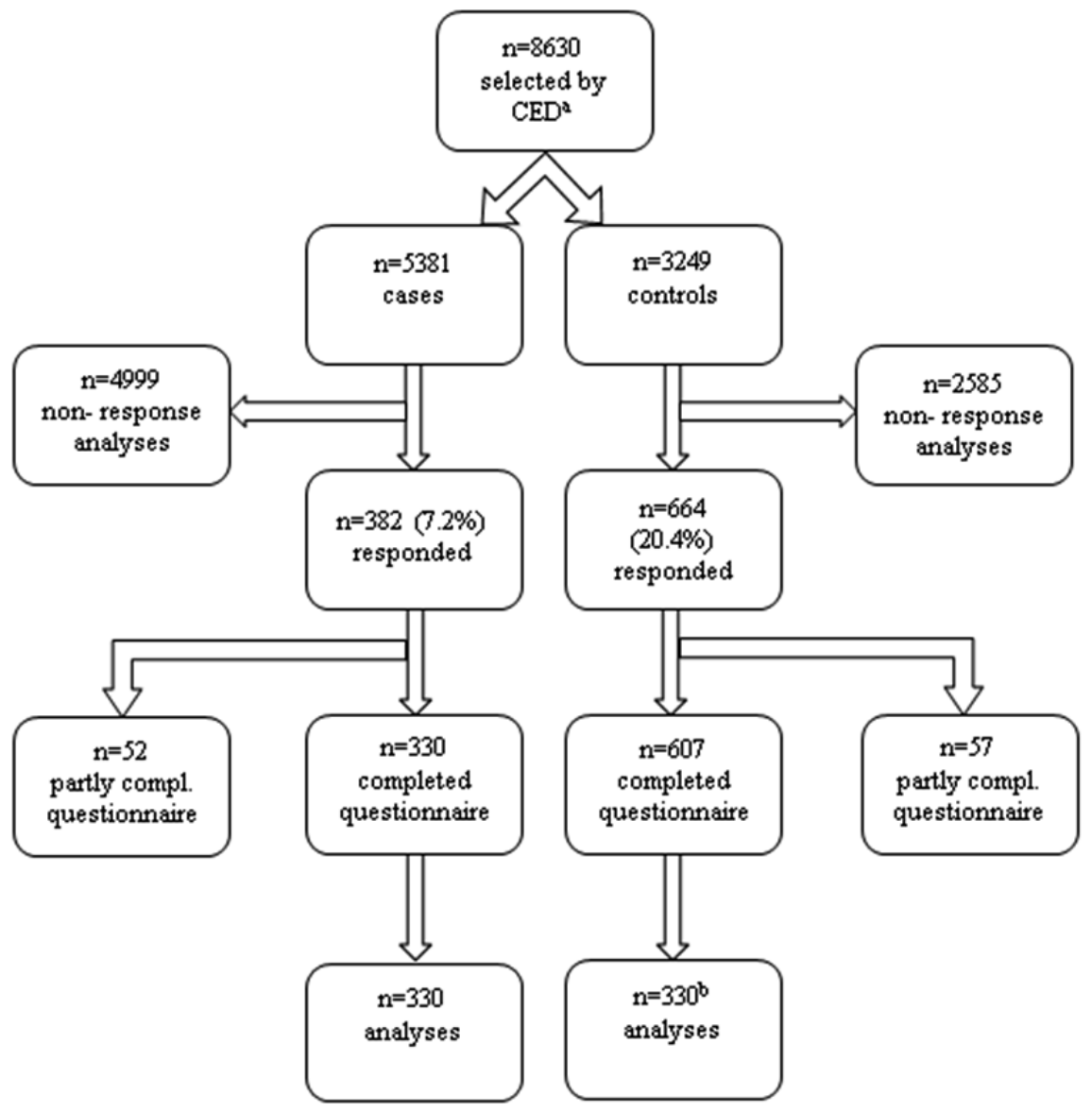

Figure 1. Inclusion flowchart.

${ }^{\mathrm{a}}$ CED = municipal department that monitors school dropout among adolescents.

${ }^{\mathrm{b}}$ randomly selected 
Table 1. Gender and covariates in relation to dropout status, separately for male and female young adults. ${ }^{a}$

\begin{tabular}{|c|c|c|c|c|c|}
\hline \multirow[b]{4}{*}{ n (\%) } & \multirow{4}{*}{$\begin{array}{l}\text { Total } \\
600 \text { (100) }\end{array}$} & \multirow{2}{*}{\multicolumn{2}{|c|}{$\begin{array}{l}\text { Male } \\
260(39.4 \%)\end{array}$}} & \multirow{2}{*}{\multicolumn{2}{|c|}{$\begin{array}{l}\text { Female } \\
400(60.6 \%)\end{array}$}} \\
\hline & & & & & \\
\hline & & \multirow{2}{*}{$\begin{array}{l}\text { Dropouts } \\
130(50.0)\end{array}$} & \multirow{2}{*}{$\begin{array}{l}\text { Controls } \\
130(50.0)\end{array}$} & \multirow{2}{*}{$\begin{array}{l}\text { Dropouts } \\
200(50.0)\end{array}$} & \multirow{2}{*}{$\begin{array}{l}\text { Controls } \\
200(50.0)\end{array}$} \\
\hline & & & & & \\
\hline AMIRS (mean/sd) & $1.6(0.5)$ & $1.9(0.5)$ & $1.9(0.5)$ & $1.5(0.5)^{*}$ & $1.4(0.4)^{*}$ \\
\hline AFIS-ISR (mean/sd) & $2.7(0.7)$ & $2.6(0.6)$ & $2.6(0.7)$ & $2.8(0.7)$ & $2.7(0.6)$ \\
\hline SR femininity (mean/sd) & $6.6(2.4)$ & $5.1(2.5)$ & $5.0(2.2)$ & $7.3(2.0)^{*}$ & $7.8(1.5)^{*}$ \\
\hline SR masculinity (mean/sd) & $5.0(2.6)$ & $6.8(2.2)$ & $6.6(1.7)$ & $4.1(2.5)$ & $3.8(2.1)$ \\
\hline Age (mean/sd) & $19.1(1.2)$ & $19.5(1.5)^{*}$ & $19.0(1.0)^{*}$ & $19.2(1.4)$ & $18.9(1.0)$ \\
\hline Non-western background & $65(9.9)$ & $18(14.0)$ & $8(6.2)$ & $30(15.0)^{*}$ & $9(4.5)^{*}$ \\
\hline One-parent family & $103(15.6)$ & $20(15.4)$ & $16(12.3)$ & $51(25.6)^{*}$ & $16(8.0)^{*}$ \\
\hline High-educated mother & $120(18.7)$ & $23(18.4)$ & $32(24.6)$ & $33(17.5)$ & $32(16.2)$ \\
\hline Intermediate-educated mother & $222(34.6)$ & $47(37.6)$ & $47(36.2)$ & $56(29.6)$ & $72(36.4)$ \\
\hline Low-educated mother & $300(43.5)$ & $55(44.0)$ & $51(39.2)$ & $100(52.9)$ & $94(47.5)$ \\
\hline High-educated father & $169(27.1)$ & $37(29.8)$ & $49(39.5)$ & $37(20.7)$ & $46(24.0)$ \\
\hline Intermediate-educated father & $183(29.4)$ & $38(30.6)$ & $38(30.6)$ & $56(31.3)$ & $51(26.6)$ \\
\hline Low-educated father & $271(43.5)$ & $49(39.5)$ & $41(29.8)$ & $86(48.0)$ & $95(49.5)$ \\
\hline
\end{tabular}

a Theoretical minimum and maximum for AMIRS $(1,4)$, AFIS-ISR $(1,5)$, self-rated femininity and self-rated masculinity $(1,10)$, age $(18,23){ }^{*} p<0.05$ according to Chi Square tests (dichotomous variables) and t-tests (continuous variables).

Among the female participants, there was a consistent pattern of curvilinear effects of all four gender variables, with intermediate values having protective effects against school dropout. The curvilinear relationship reached statistical significance $(p<0.05)$ for AFIS and masculinity (not tabulated). Table 2 shows the lower odds of dropout for the female young adults with intermediate values on all four gender variables. Simultaneously, the odds ratios show that a high self-reported masculinity increased the odds of school dropout for female participants (OR 2.1, 95\% Cl 1.1-4.1). We found no significant interaction effects between the different gender variables on school dropout for the female participants (not tabulated). 
Table 2. Odds ratios (95\% confidence interval) of dropout among female young adults, by gender, adjusted for age, ethnic background, educational level of the parents, and family composition.

\begin{tabular}{lll}
\hline & OR & $95 \% \mathrm{Cl}$ \\
\hline AMIRS & & \\
Low $^{\text {a }}$ & 1.0 & \\
Intermediate & 1.0 & $0.6-1.6$ \\
High & 1.5 & $0.8-2.6$ \\
AFIS & & \\
Low $^{\text {a }}$ & 1.0 & \\
Intermediate & 0.9 & $0.5-1.5$ \\
High & 1.2 & $0.7-2.0$ \\
Femininity & & \\
Low & \\
Intermediate & 1.0 & \\
High & $0.3^{* *}$ & $0.1-0.7$ \\
Masculinity & $0.3^{* *}$ & $0.1-0.7$ \\
Low & & \\
Intermediate & & $0.4-1.1$ \\
High & 1.0 & $1.1-4.1$ \\
\hline
\end{tabular}

${ }^{a}$ Reference group ${ }^{*} p<0.05,{ }^{* *} p<0.01$

As regards the male participants, Figure 2A shows that a high AMIRS score significantly increased the odds of dropout for men who considered themselves highly masculine and also for men who scored low on both gender variables ( $p=0.04$ for interaction). The interaction effect between the AMIRS and AFIS scores was marginally significant (Figure 2B): a high score on AMIRS increased the odds of dropout when the AFIS score was low $(p=0.06)$. Figure $2 C$ shows that male participants who considered themselves low on masculinity had significantly higher odds of dropout when they also considered themselves highly feminine $(p=0.01)$. Male participants who considered themselves highly feminine and scored low on the AMIRS also had higher odds of dropout ( $p=0.08$ ) (Figure 2D). Interactions between masculinity and AFIS and between femininity and AFIS were not statistically significant (Figures $2 \mathrm{E}$ and $2 \mathrm{~F}$ ). 
A

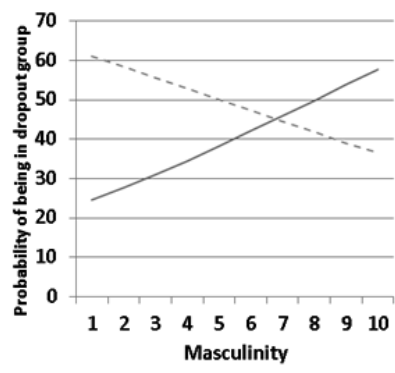

AMIRS*masculinity, $p=0.04$

C

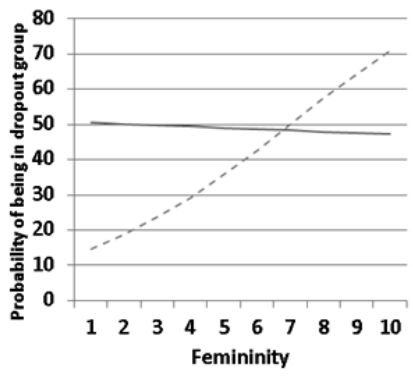

Masculinity*femininity, $p=0.01$

E

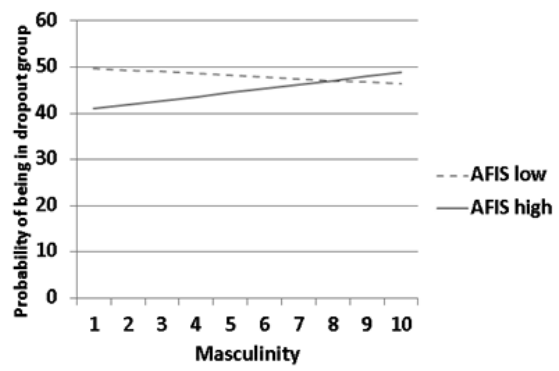

AFIS*masculinity, $p=0.70$
B

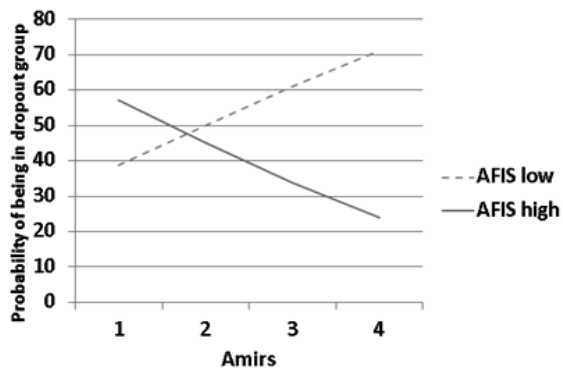

AFIS*AMIRS, $p=0.06$

D

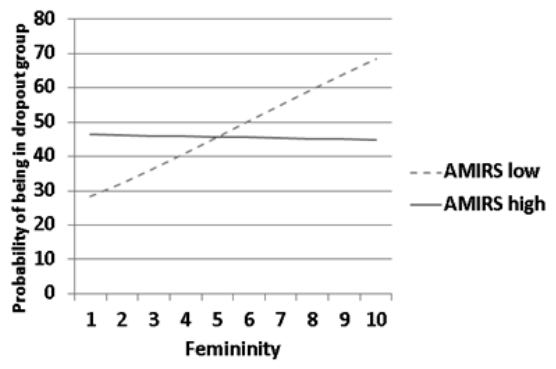

AMIRS*femininity, $p=0.08$

F

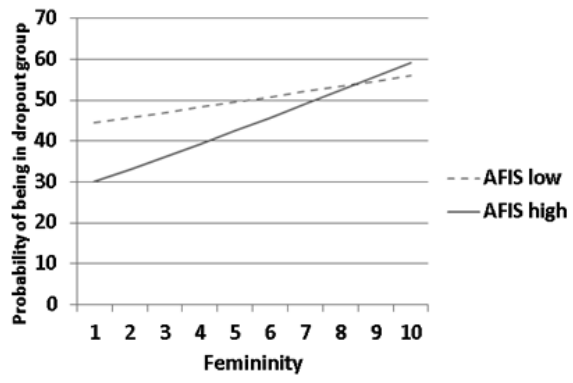

AFIS* femininity, $p=0.48$

Figure 2. Odds ratios (95\% confidence interval) of dropout among male young adults, by gender, adjusted for age, ethnic background, educational level of the parents, and family composition. Separate estimates for high $($ mean+1sd) and low (mean-1sd) scores on the other gender variable. 


\section{Discussion}

Our findings show that school dropout and gender are related and that this relationship differs for female and male young adults. For young women, the relation is curvilinear, as an intermediate score on the gender variables tended to protect best against dropout. A high masculinity score particularly increased the odds of dropout in young women. For young men, the gender effect was complex, as it especially presented itself in terms of interactions. The highest odds of dropout were found among highly masculine young men who simultaneously had strong normative beliefs about male peer relationships and among young men who considered themselves simultaneously high on femininity and low on masculinity.

For young women the associations between gender and school dropout show a group who might be protected against school dropout and another one that has an increased risk. One potential explanation for this is that young women with both masculine and feminine traits may possess a wider variety of skills, enhancing their ability to cope with different situations and life's problems $(5,14)$. This may explain our finding that an intermediate score on masculinity decreased the odds of school dropout for young women. Second, rather than increasing the dropout odds (25), hyper-femininity protected women against school dropout while high masculinity scores increased their odds of dropout. Performing well at school is considered feminine and increases popularity among girls (16). By contrast, antisocial behavior and aggression in highly masculine girls might lead to social exclusion $(16,33)$. Particularly, lonely girls with low selfesteem could more easily feel socially isolated and be more inclined to join "gangs" (34, 35). In the masculine street culture, young people base their self-worth and identity on performance and competition. Identifying with the masculine norms and values of street culture will result in tougher behavior (36). Going to school and doing your homework is not cool, which results in hyper-masculine young women having higher odds of school dropout.

The young men's pattern of association can best be characterized as involving two risk groups. First, young men who were hyper-masculine were more likely to drop out of school, which is similar to what others have found (25). Highly masculine young men with a normative view of dominance in peer relationships (probably providing them with peer status) had higher odds of dropout. Tough boys are popular (16) and when they demonstrate deviant behavior they might earn the respect of their peers (15). However, striving for a masculine self-image is associated with anti-social behavior (33), which might underlie the higher odds of dropping out (21). Second, young men with many feminine traits and at the same time few masculine ones are also at risk for school dropout. Men who do not conform to the normative male gender role run the risk of being stigmatized and bullied or even rejected by their peers or their family (37). To avoid social exclusion, these men may tend to behave in a stereotypical masculine way (33) which in turn may lead to problem behavior (18). Other studies have shown that 
people with a homosexual orientation are more likely not to conform to gender roles even at an early age $(37,38)$. Our study yielded no information on whether homosexuality plays a role, but it is possible to experience difficulties if you do not meet heterosexuality norms. Besides, coming out is often a difficult decision with a risk of being discriminated, stigmatized or socially rejected. There is therefore a need for greater acceptance and understanding of variations in gender roles and beliefs. Future research should investigate in greater depth the role gender plays in relations with peers, parents and teachers.

Our findings underscore the importance of gender in public health research, but given the complexity of relationships between gender traits and gender beliefs (curvilinearity in women and interactions in men), the concept and assessment of gender require further refinement and research.

Our study has three strengths. First, sex and gender have rarely been examined in public health research (39). Research on gender beliefs in the process leading to school dropout has been scarce and limited to masculinity in boys and femininity in girls. Our study emphasises the relevance of additionally examining masculinity in females and femininity in males. Second, although categorizing masculinity and femininity is problematic, our study shows that neither male nor female young adults have a dichotomous perception of gender, but that they do assign masculine and feminine traits to themselves. Moreover, although on average boys assigned higher masculinity traits to themselves and girls assigned higher femininity traits to themselves, boys and girls seemed to have taken this question quite seriously and did not self-stereotype. Third, to avoid selection bias, we sought a control group which was highly similar to the cases at the start of the school year 2010-2011. The reported odds ratios thus only apply to an already initially disadvantaged population (at the age of 18 to 23, both cases and controls already should have attained their starting qualification). Simultaneously, we might have underestimated the associations compared to a study using all possible controls.

Three limitations need to be mentioned. First, the non-response rate was substantial. For both participants and non-participants, we had information on dropout status, age, sex and socioeconomic status from external socioeconomic data at zip-code level obtained from Statistics Netherlands (40). Non-participants were more likely to be men, dropouts (cases) and living in areas with cheaper houses, and were slightly older than the participants. In the total group (including the eventual non-participants), men were more likely to have dropped out than women, indicating that the absence of a sex effect on dropout in the final sample is an underestimate. As we have no information on gender beliefs for the non-participants, it is unclear how the above pattern of nonparticipation might have affected the odds ratios. Second, the questionnaire was administered after participants had dropped out of school, so the answers may have been biased by recall. However, the questions about gender beliefs and gender traits were introduced by explicitly asking the participants to think of the period when they were 16 , which is prior to dropout. Third, gender ideologies may play a role in completing the 
questionnaire. For instance, if we had asked the respondents to rate the same beliefs without labeling them as masculine and feminine, male participants with strong normative beliefs could have assigned themselves more caring traits (29). It is possible that such reporting bias may have led to underestimated odds of dropout for feminine men and masculine women in particular.

\section{Conclusion}

Our findings justify the inclusion of gender beliefs in the explanation of school dropout. There are some young men and women who are 'too cool for school'. Therefore, preventive child and youth healthcare professionals, who offer routine health examinations and anticipatory guidance to all children between birth and 19 years, should assess gender beliefs and behaviors in each adolescent and discuss whether this affects their parent and peer relations, school engagement or leisure activities. Subsequently, they should follow up and monitor the adolescents' interpersonal relations, behavior problems and school engagement, and in case the adolescents are at risk for dropout, they should intervene.

\section{Key points}

- In contrast to the findings on the influence of sex, it is unclear how gender beliefs relate to school dropout in both young men and women.

- Using Dutch case-control data, some young men and women appeared 'too cool for school', as they were highly masculine.

- Men who consider themselves high on femininity and low on masculinity are also more likely to drop out.

- Gender associations were complex, as curvilinearity was detected in women and effect-modification in men.

- In order to prevent dropout, public health professionals might start considering to assess and monitor gender beliefs and to think about appropriate interventions. 


\section{References}

1. Freudenberg N, Ruglis J. Reframing school dropout as a public health issue. Prev Chronic Dis. 2007 Oct;4(4):1-11.

2. Suhrcke $M$, de Paz Nieves $C$. The impact of health and health behaviours on educational outcomes in high-income countries: a review of the evidence. Copenhagen: WHO Regional Office for Europe; 2011.

3. Mäki N, Martikainen P, Eikemo T, Menvielle G, Lundberg O, Östergren O, et al. Educational differences in disability-free life expectancy: a comparative study of long-standing activity limitation in eight European countries. Soc Sci Med. 2013;94:1-8.

4. Oliffe JL, Greaves LJ. Designing and Conducting Gender, Sex, and Health Research. Thousand oaks CA: Sage Publishers 2012.

5. Howard JA, Hollander J. Gendered situations, gendered selves. Oxford: AltaMira Press; 2000.

6. Women and Gender in Research. European Commision. Research \& Innovation - Sience in Society; Available from: http://ec.europa.eu/research/science-society/index.cfm? fuseaction=public.topic\&id=1253\&lang=1.

7. Bem SL. The measurement of psychological androgyny. J Consult Clin Psychol. 1974;42(2):155-62.

8. Spence JT, Helmreich R, Stapp J. Ratings of self and peers on sex role attributes and their relation to selfesteem and conceptions of masculinity and femininity. J Pers Soc Psychol. 1975;32(1):29-39.

9. Strough J, Leszczynski J, Neely TL, Flinn JA, Margrett J. From Adolescence to Later Adulthood: Femininity, Masculinity, and Androgyny in Six Age Groups. Sex Roles. 2007;57(5-6):385-96.

10. Verdonk $P$, Seesing $H$, de Rijk A. Doing masculinity, not doing health? a qualitative study among dutch male employees about health beliefs and workplace physical activity. BMC Public Health. 2010;10(1):712.

11. Connell R, Messerschmidt J. Hegemonic masculinity. Rethinking the concept. Gender Soc. 2005;19:829 - 59.

12. Twenge J. Changes in masculine and feminine traits over time: A meta-analysis. Sex Roles. 1997;36(56):305-25.

13. Wilde A, Diekman AB. Cross-Cultural Similarities and Differences in Dynamic Stereotypes: A Comparison Between Germany and the United States. Psychol Women Q. 2005 June 1, 2005;29(2):188-96.

14. Murray C, Greenberg MT. Children's relationship with teachers and bonds with school. An investigation of patterns and correlates in middle childhood. J Sch Psychol. 2000;38(5):423-45.

15. Rodkin PC, Farmer TW, Pearl R, Acker RV. They're Cool: Social Status and Peer Group Supports for Aggressive Boys and Girls. Soc Dev. 2006;15(2):175-204.

16. Adler PA, Kless SJ, Adler P. Socialization to Gender Roles: Popularity among Elementary School Boys and Girls. Sociol Educ. 1992;65(3):169-87.

17. Muehlenhard C, Peterson Z. Distinguishing Between Sex and Gender: History, Current Conceptualizations, and Implications. Sex Roles. 2011;64(11-12):791-803.

18. Pleck J, Sonenstein F, Ku L, editors. Masculinity ideology and its correlates. Newbury Park CA: Sage Publications; 1993.

19. Staff J, Kreager DA. Too Cool for School? Violence, Peer Status and High School Dropout. Soc Forces. 2008;87(1):445-71.

20. Alatupa S, Pulkki-Råback L, Hintsanen M, Mullola S, Lipsanen J, Keltikangas-Järvinen L. Childhood Disruptive Behaviour and School Performance across Comprehensive School: A Prospective Cohort Study. Psychology 2011;2(6):542-51.

21. Ensminger $M$, Slusarcick $A$. Paths to high school graduation or dropout: A longitudinal study of a first grade cohort. Sociol Educ. 1992;65:95-113.

22. Ueno K, McWilliams S. Gender-Typed Behaviors and School Adjustment. Sex Roles. 2010;63(7):580-91.

23. Theunissen M-J, Griensven van I, Verdonk P, Feron F, Bosma H. The early identification of risk factors on the pathway to school dropout in the SIODO study: a sequential mixed-methods study. BMC Public Health. 2012;12(1):1033.

24. Ministery of Education Culture and Science. Dossier aanval op schooluitval [Drive to Reduce Drop-out Rates]. 2008; Available from: http://www.aanvalopschooluitval.nl/beleid/aanval-op-schooluitval-20082011. 
25. Eurostat. Glossary:Early leaver from education and training. Luxembourg: European Union; 2013 [cited 2013 17-11-2013]; Available from: http://epp.eurostat.ec.europa.eu/statistics_explained/index.php/ Glossary:Early_school_leaver.

26. Chu JY, Porche MV, Tolman DL. The Adolescent Masculinity Ideology in Relationships Scale: Development and Validation of a New Measure for Boys. Men Masc. 2005;8(1):93-115.

27. Tolman DL, Porche MV. The Adolescent Femininity Ideology Scale: Development and validation of a new measure for girls. Psychol Women Q. 2000;24(4):365-76.

28. Spence JT, Buckner CE. Instrumental and expressive traits, trait stereotypes, and sexist attitudes. What do they signify? Psychol Women Q. 2000;24(1):44-53.

29. Pedhazur EJ, Tetenbaum TJ. Bem Sex Role Inventory: A theoretical and methodological critique. J Pers Soc Psychol. 1979;37(6):996-1016.

30. Statistics Netherlands. Dutch population. 2012 [updated 22 februari 2012; cited 20132 dedember]; Available from: http://statline.cbs.nl/StatWeb/publication/?VW=T\&DM =SLNL\&PA=70748NED\&D1=0,2,4, $16,18,20,22,24 \& D 2=a \& D 3=0 \& D 4=a \& D 5=\mid \& H D=090707-1905 \& H D R=T \& S T B=G 4, G 2, G 1, G 3$.

31. Shavers VL. Measurement of socioeconomic status in health disparities research. J Natl Med Assoc. 2007 September;99(9):1013-23.

32. Volksgezondheid Toekomst Verkenning Nationaal Kompas Volksgezondheid [The National Public Health Compass]. Bilthoven: RIVM; 2013 [28 maart 2013]; 4.11:[Available from: http://www.nationaalkompas.nl/ bevolking/scholing-en-opleiding/indeling-opleidingsniveau/.

33. Hay I. Gender Self-concept Profiles of Adolescents Suspended from High School. J Child Psychol Psychiatry. 2000;41(3):345-52.

34. Esbensen F-A, Deschenes EP, Winfree LT. Differences between Gang Girls and Gang Boys: Results from a Multisite Survey. Youth Soc. 1999;31(1):27-53.

35. Wang JZ. Female Gang Affiliation: Knowledge and Perceptions of At-Risk Girls. Int J Offender Ther Comp Criminol. 2000;44(5):618-32.

36. Klein MW, Weerman FM, Thornberry TP. Street Gang Violence in Europe. European Journal of Criminology. 2006;3(4):413-37.

37. Rieger G, Linsenmeier JAW, Gygax L, Bailey JM. Sexual orientation and childhood gender nonconformity: Evidence from home videos. Dev Psychol. 2008;44(1):46-58.

38. Bailey JM, Zucker KJ. Childhood sex-typed behavior and sexual orientation: A conceptual analysis and quantitative review. Dev Psychol. 1995;31(1):43-55.

39. Lawrence K, Rieder A. Methodologic and ethical ramifications of sex and gender differences in public health research. Gend Med. 2007;4, Supplement 2(0):S96-S105.

40. Statistics Netherlands. Kerncijfers postcodegebieden 2004. Voorburg2006 [cited 201320 August]; Available from: http://www.cbs.nl/nl-NL/menu/themas/dossiers/nederland-regionaal/cijfers/incidenteel/ maatwerk/kerncijfers-postcodegebieden-2004.htm. 



\section{Chapter 5}

\section{Low control beliefs in relation to school dropout and poor health:}

findings from the SIODO case-control study

Published as:

Bosma $\mathrm{H}$, Theunissen M-J, Verdonk P, Feron F. Low control beliefs in relation to school dropout and poor health: findings from the SIODO case-control study. BMC Public Health. 2014;14(1):1237. 


\section{CHAPTER 5}

\section{Abstract}

\section{Background}

There is cumulating evidence that health is compromised through adverse socioeconomic conditions negatively affecting how people think, feel, and behave. Low control beliefs might be a key mechanism. The reversed possibility that low control beliefs might set people on a pathway towards adverse socioeconomic and health-related outcomes is much less examined.

\section{Methods}

A case-control design was used, consisting of 330 cases who dropped out of school in the 2010-2011 school year and 330 controls who still attended school at the end of that year. The respondents, aged between 18 and 23, came from Eindhoven and surrounding areas in the south-east of The Netherlands. A questionnaire asked for current health status, recalled socioeconomic and social background, and recalled control beliefs (mastery and general self-efficacy). Logistic regression analyses were used.

\section{Results}

Recalls of low mastery and low self-efficacy were strongly related to both dropout and less than good health. Low socioeconomic background was also associated to odds of dropout, but did not confound or moderate the associations of low control beliefs with dropout and health. Odds ratios of dropout and less than good health indicated at least twice the odds of a poor outcome with recalls of low control beliefs.

\section{Conclusions}

Independent of the socioeconomic background, low control beliefs are related to heightened odds of both poor health and school dropout. Individual differences in control beliefs might thus be as fundamental as socioeconomic conditions in generating life-course socioeconomic and health-related pathways. Although the findings should first be cross-validated in prospective studies, public health professionals working with youth might already start considering early interventions in youth with all too fatalistic and powerless mind-sets. 


\section{Background}

Low control beliefs have often been studied in the context of the higher risks of disease and premature mortality in lower socioeconomic status groups (e.g. 1-4). Control beliefs can be defined as a person's belief that he or she is able to actually perform a (desired) action or behaviour (self-efficacy) and the belief that his or her actions matter for outcomes and events (mastery) (5). Low control beliefs are related to poor health outcomes, through either negatively affecting health behaviours or having direct repercussions for physiological functioning. Some have labelled low control beliefs as powerlessness or "socialised fatalism" (6). The latter emphasises the embedding in the socioeconomic environment $(4,7)$. Low income hampers the number of available options; control over what to purchase is thus restricted. Similarly, long-term low control and autonomy at work, which is common in lower socioeconomic status groups $(8,9)$, might hinder the development of self-directed behaviours and planning. Low control beliefs have thus been found partially rooted in socioeconomic conditions.

Much less examined is the reversed possibility of low control beliefs negatively affecting socioeconomic attainment (e.g. school dropout) and poor health (2). High control beliefs might promote social mobility, as people with such beliefs are confident both about their ability to perform well at school and about the future benefits of a higher educational career $(10,11)$. The question now is whether control beliefs influence socioeconomic attainment processes and later health and whether this influence is independent of social differences, as indicated by prior socioeconomic and social conditions. An independent influence of control beliefs would suggest that individual differences might be as fundamental as social differences for life-course socioeconomic and health-related pathways. This possibility of selection via individual personality characteristics has insufficiently been examined in the field of socioeconomic differences in health (12).

Using Dutch case-control data on 330 school dropouts and 330 controls still attending school, we set out to examine whether adolescent low control beliefs affect school dropout and poor health in young adulthood. The embedding in the socioeconomic background during upbringing is studied by examining the association of parental socioeconomic and social characteristics during upbringing with control beliefs. Simultaneously, it is examined whether low control beliefs relate to school dropout and poor health, independent of the socioeconomic and social background. The purpose of the study is to help with optimizing interventions aimed at tackling socioeconomic differences in health and to give public health professionals working with youth more personalised tools aimed at a timely prevention of school and health-related difficulties. 


\section{Method}

\section{Study population}

This study is part of the Dutch SIODO study, a sequential mixed-methods study, focusing on the early identification of risk factors on the pathway to school dropout (13). The current paper is using the case-control quantitative data from SIODO. In November 2011, the municipal compulsory education department of the city of Eindhoven selected all young adults aged 18-23 years who had not yet met the Dutch minimum educational requirement, the so-called "basic qualification", at the start of the 2010-2011 school year. This minimum qualification (for making a good entry on the labour market) is equivalent to higher general secondary education, pre-university education or intermediate vocational education, Level 2. At this age, they normally should have obtained this required qualification. A proportion of these young adults remained in school during the 2010-11 school year to obtain this qualification (the controls), while others dropped out of school during that year without qualification (the cases). Cases and controls being so similar at the start of the 2010-11 school year was supposed to avoid selection bias.

On average 1.5 year after the start of the 2010 - 2011 school year, we sent a selfadministered questionnaire with an informed consent form to the eligible young adults. The questionnaire contained questions on the current health status, recalled control beliefs, recalled socioeconomic background, and potential confounders.

The power calculation for a retrospective study with a dichotomous outcome variable indicated that 290 cases and 290 controls would yield an 80\% power to detect an odds ratio of 1.75 at a $\alpha$-level of $5 \%$ for an exposure of 0.2 and a ratio of cases to controls of $1(14,15)$. We had to send 8,630 questionnaires to recruit 1,049 possible participants, among which 330 cases. The 330 controls were randomly selected from the remaining group of participating controls. Approval for conducting this study was granted by the Medical Ethics Committee of Maastricht University (METC 11-4-099, decision 2208-2011). More detail on the SIODO study can be found elsewhere (13).

\section{Measures}

\section{Dropout status and current health status}

Dropout status in the school year 2010-2011 was determined by the compulsory education department as defined in the previous paragraph. The subsequent questionnaire asked for the current health status. This was measured by asking how healthy the young adults currently considered themselves (varying from 1: not healthy at all to 10: very healthy). This variable was dichotomised by assigning respondents scoring lower than 7 to the less than good health category $(n=81(25 \%)$ in the case group and $n=32(10 \%)$ in the control group). 


\section{Recalled low control beliefs}

For both mastery and general self-efficacy, the respondents were asked to think of the time period when they were 16 (prior to the dropout). Mastery was measured by computing the mean of the seven items of the Pearlin and Schooler scale (0: low and 5: high mastery) (Cronbach's $\alpha=0.84$ ) (16). The introduction of this questionnaire asked the respondents to report what was most applicable for them when they were 16 . Two example items are: "I have little control over things that happen to me" (to be reverse coded) and "I can do just about anything I really set my mind to do". General selfefficacy was measured by computing the mean on the 16 items of Sherer's general selfefficacy scale (0: low and 5: high self-efficacy) (Cronbach's $\alpha=0.90)$ (17). These questions had the same introduction as mastery (asking them to recall their psychological profile at the age of 16). Two example items are: "If something looks too complicated, I will not even bother to try it" (to be reverse coded) and "When I make plans, I am certain I can make them work". Both variables were categorised into thirds using tertiles.

\section{Socioeconomic and social background}

Socioeconomic background was based on the mean of the standardised scores for educational level of the father and the mother separately and four questions on material and social deprivation. Education had the following ordinal categories: no primary education, primary education only, lower vocational education, intermediate general secondary education, intermediate vocational education, higher general secondary education, higher vocational education, and university education. Separate for the primary and secondary school period, the material deprivation items asked for how often there was too little money for food or for replacing clothes or shoes (never, sometimes, regularly, always). Separate for the primary and secondary school period, the social deprivation items asked for how often there was too little money for joining a (sports) club or going on a school trip (never, sometimes, regularly, always). The resulting composite variable for socioeconomic background was categorised into thirds using tertiles. Ethnic background, looking at both the respondents' and their parents' country of birth, was dichotomous, as respondents with a western migration background were too small in numbers (0: autochthonous/western background and 1: non-western background). Family composition during primary school was defined as either living with both parents or living with one parent only.

\section{Other confounders}

Both sex and age at the start of the school year were used as covariates.

\section{Statistical analyses}

First, the associations of the socioeconomic, ethnic, and family background with dropout were analysed using cross-tabulations and related chi $^{2}$-tests (Table 1 ). The associations of 
the background variables with less than good health and low control beliefs were examined in the case and control group, separately (Table 2). Second, the association of low control beliefs with dropout was also examined by chi ${ }^{2}$-tests. Logistic regression analyses were used to examine the same association, controlling for age and sex, and additionally for the socioeconomic background, ethnic background, and family composition (Table 3). To examine whether low control beliefs were equally predictive of school dropout in different socioeconomic backgrounds, multiplicative interactions between control beliefs and socioeconomic background were separately tested. Third, the association of low control beliefs with current health was examined by chi ${ }^{2}$-tests in the case and control group, separately. Logistic regression analyses were used to control for age and sex, and additionally for the socioeconomic background, ethnic background, and family composition (Table 4). Multiplicative interactions between socioeconomic background and low control beliefs were also tested. Sensitivity analyses were done to study the robustness of the findings when using the continuous versions of all variables (and linear regression for current health) and when additionally controlling for the educational level of the first class in secondary school (having four ordinal categories).

\section{Results}

Table 1 shows that the cases, compared to controls, more often came from lower socioeconomic backgrounds (38.2\% vs. $28.8 \%$ ), non-western backgrounds (14.6 vs. 5.2 ), and one-parent families (21.6 vs. 9.7).

Table 1. Associations of socioeconomic background, ethnic background, family composition and sex with casecontrol status (column percentages). ${ }^{\text {a }}$

\begin{tabular}{lll}
\hline & $\begin{array}{l}\text { Controls } \\
(\mathrm{n}=330) \\
\%\end{array}$ & $\begin{array}{l}\text { Cases } \\
(\mathrm{n}=330)\end{array}$ \\
& $\%$ & $*$ \\
\hline $\begin{array}{l}\text { Socioeconomic background } \\
\text { High }\end{array}$ & 36.4 & 31.5 \\
Intermediate & 34.8 & 30.3 \\
Low & 28.8 & 38.2 \\
Ethnic background & & $*$ \\
Autochthonous background ${ }^{b}$ & 94.8 & 85.4 \\
Non-western background & 5.2 & 14.6 \\
Family composition & & $*$ \\
Two parents & 90.3 & 78.4 \\
One parent & 9.7 & 21.6 \\
Sex & & 60.6 \\
Women & 60.6 & 39.4 \\
Men & 39.4 & \\
\hline
\end{tabular}

* $p \leq 0.05$ (chi ${ }^{2}$-test). ${ }^{a}$ Mean age differed significantly between cases (19.3 years) and controls (19.0 years)

${ }^{\mathrm{b}}$ Including a western migration background. 
In the case group, low socioeconomic background was related to less than good health (30.4 vs. 17.3 ) and low mastery recalls (54.8 vs. 32.7) (Table 2). In the control group, adolescents from one-parent families much more often reported low mastery than adolescents from two-parent families (53.1 vs. 22.5). All other associations were statistically not significant.

Table 2 Associations of socioeconomic background, ethnic background, family composition and sex with less than good health, low mastery, and low self-efficacy in cases and controls, separately (row percentages). ${ }^{a}$

\begin{tabular}{|c|c|c|c|c|c|c|}
\hline & \multicolumn{2}{|c|}{$\%$ less than good health } & \multicolumn{2}{|c|}{$\%$ low mastery $^{\mathrm{b}}$} & \multicolumn{2}{|c|}{$\%$ low self-efficacy ${ }^{b}$} \\
\hline & Cases & Controls & Cases & Controls & Cases & Controls \\
\hline Socioeconomic background & $*$ & & $*$ & & & \\
\hline High & 17.3 & 10.8 & 32.7 & 23.3 & 36.5 & 27.5 \\
\hline Intermediate & 25.3 & 6.1 & 38.0 & 24.3 & 37.0 & 22.6 \\
\hline Low & 30.4 & 12.6 & 54.8 & 29.5 & 47.6 & 24.2 \\
\hline \multicolumn{7}{|l|}{ Ethnic background } \\
\hline Autochthonous background ${ }^{b}$ & 25.3 & 9.6 & 42.0 & 25.2 & 39.9 & 25.2 \\
\hline Non-western background & 21.7 & 11.8 & 45.8 & 29.4 & 47.9 & 17.6 \\
\hline Family composition & & & & $*$ & & \\
\hline Two parents & 24.9 & 9.4 & 39.5 & 22.5 & 36.0 & 25.2 \\
\hline One parent & 24.3 & 12.5 & 53.5 & 53.1 & 59.2 & 21.9 \\
\hline \multicolumn{7}{|l|}{ Sex } \\
\hline Women & 29.3 & 8.0 & 45.5 & 23.5 & 41.0 & 26.0 \\
\hline Men & 17.7 & 12.3 & 38.5 & 28.5 & 40.8 & 23.1 \\
\hline
\end{tabular}

* $p \leq 0.05$ (chi ${ }^{2}$-test). ${ }^{a}$ Mean age differed significantly between those with (19.4 years) and without (18.9 years) less than good health. ${ }^{b}$ Mastery and general self-efficacy were left in three categories, but only the percent in the lowest tertile is shown. ${ }^{c}$ Including a western migration background.

Recalls of low mastery and self-efficacy were strongly related to odds of school dropout (Table 3). For example, adolescents with recalls of low mastery had $144 \%$ higher odds of dropout (odds ratio $=2.44$ ) compared with adolescents with high mastery recalls $(95 \%$ $\mathrm{Cl}$ : 1.66-3.59). Controlling for the socioeconomic, ethnic, and family background hardly affected these odds ratios. 


\section{CHAPTER 5}

Table 3 Odds ratios (95\% confidence intervals) of school dropout by recalled low control beliefs, adjusted for age, sex (model 1), and additionally adjusted for family composition, and ethnic and socioeconomic background (model 2).

\begin{tabular}{lllll}
\hline & $\begin{array}{l}\text { Controls }(\mathrm{n}=330) \\
\%\end{array}$ & $\begin{array}{l}\text { Cases }(\mathrm{n}=330) \\
\%\end{array}$ & Model 1 & Model 2 \\
\hline Mastery & $*$ & & \\
High & 40.0 & 26.7 & 1.00 & 1.00 \\
Medium & 34.5 & 30.6 & $1.28(0.87-1.88)$ & $1.27(0.86-1.88)$ \\
Low & 25.5 & 42.7 & $2.44(1.66-3.59)$ & $2.15(1.44-3.20)$ \\
Self-efficacy & & $*$ & & \\
High & 39.4 & 26.1 & 1.00 & 1.00 \\
Medium & 35.8 & 33.0 & $1.39(0.95-2.04)$ & $1.30(0.88-1.92)$ \\
Low & 24.8 & 40.9 & $2.51(1.70-3.71)$ & $2.24(1.50-3.35)$ \\
\hline
\end{tabular}

$* \mathrm{p} \leq 0.05$ (chi ${ }^{2}$-test); percentages are column percentages.

In both the case and control group, the associations of recalled low mastery and low self-efficacy with less than good current health were also substantial (Table 4). After control for the socioeconomic, ethnic, and family composition background, odd ratios indicated a four times higher odds of less than good health for adolescents recalling low control beliefs (e.g. odds ratio $=4.10(95 \% \mathrm{Cl}$ : 1.48-11.4) for mastery in the control group). The adjustment for socioeconomic, ethnic, and family composition background did not strongly affect the odds ratios (comparing model 1 and 2). Confidence intervals were wide.

Results of analyses with the original continuous variables, including linear regressions with the health status outcome (Appendix 1), showed a similar pattern of findings. There were no significant interactions, indicating that recalled low control belief measures are similarly related to poor current health and dropout in all three socioeconomic groups. A similar absence of interactions was detected for ethnic background and family composition. Additional control for the first class level of secondary school that pupils attended did not attenuate the odds ratios for low mastery and low self-efficacy, primarily because of the absence of an association between school level and both mastery and self-efficacy. 
LOW CONTROL BELIEFS, SCHOOL DROPOUT AND POOR HEALTH

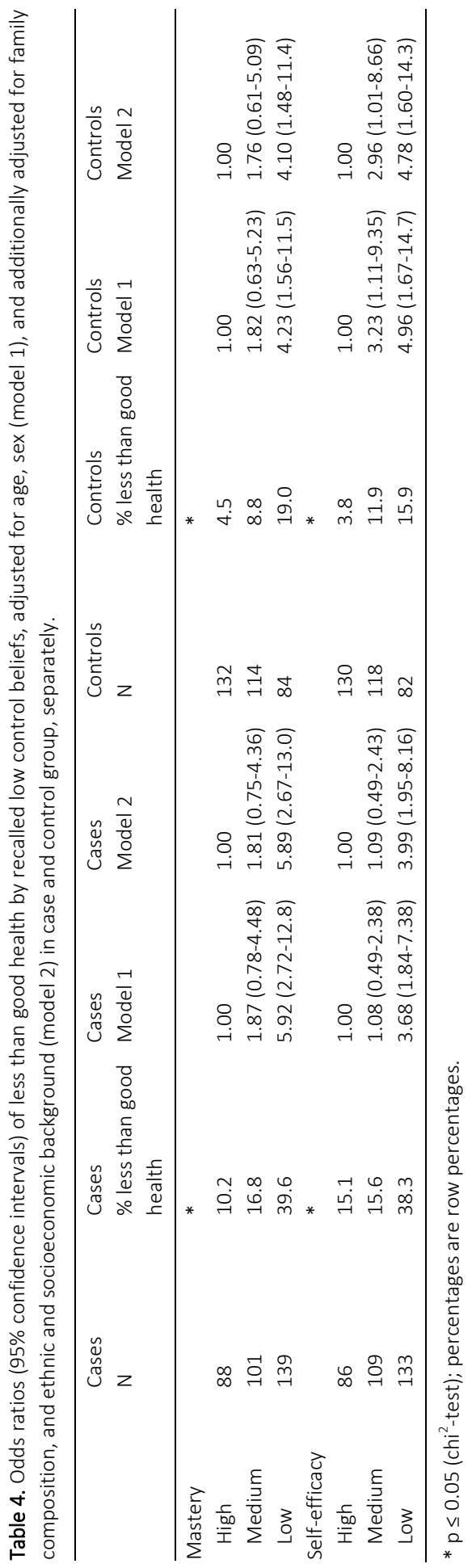




\section{Discussion}

In this group of 18 to 23 year old Dutch men and women, we found that recalls of low mastery and low self-efficacy were strongly associated with school dropout and less than good health. A lower socioeconomic background, as indicated by measures of recalled relative deprivation and parental education, was also related to school dropout, as was a non-western parental background and coming from a one-parent family. The socioeconomic and social background characteristics did neither confound nor moderate the association of low control beliefs with school dropout and less than good health, which underlines the independent association of low control beliefs with socioeconomic and health-related, life-course pathways.

Some limitations should be discussed. The first two limitations regard the casecontrol design and the cross-sectional questionnaire. First, we cannot fully exclude the possibility of dropout having affected the low control beliefs (as reported in the posterior questionnaire). Additionally, recall bias could have occurred, when the cases (compared to the controls) had a better remembrance or even reported more prior problems than actually occurred. To avoid these possibilities as much as possible, the questions on low control beliefs were introduced by explicitly asking the respondents to think of the period when they were 16, which was prior to any dropout. Second, the possibility of poor current health affecting the reports of the measures of low control beliefs in the same questionnaire was hopefully also addressed by asking for control beliefs at the age of 16 (and for current health). Dropout affecting the current health status and biasing the estimates of the association between low control beliefs and health was addressed by separate analyses of health status in the case and control group. However, to truly avoid both the first and second limitation, dedicated longitudinal studies are needed that allow a more in-depth and valid unravelling of the causal mechanisms related to socioeconomic attainment and health.

Third, non-response was substantial, as we had to send 8,630 questionnaires to recruit 1049 possible participants (12 percent). The low response rate is related to the sample, and particularly the cases, being a hard-to-reach-group. Non-participation was higher in cases (compared with controls), in boys, and in those living in areas with cheaper houses (Appendix 2). This may have resulted in an underestimation of the influence of socioeconomic conditions. In the absence of information on differential nonparticipation according to levels of control beliefs, we do not know whether and how the association of low control beliefs with dropout and health was affected by nonparticipation. Finally, both cases and controls normally should have had obtained their "basic qualification" already. This increased the similarity in the target population and thus decreased the possibility of selection bias, but might simultaneously have led to underestimated associations of determinants with school dropout. Future studies should examine the associations with a longitudinal design including all possible cases and controls. 
Many studies have conceived of low control beliefs as mediators, rather than as confounders, in the association between low socioeconomic status and poor health $(2,10,11)$. Our findings suggest the importance of thinking beyond "mediation" and of looking at earlier life individual differences (also within socioeconomic groups) as partial driving forces for social mobility, future health, and the development of socioeconomic differences in health in young adulthood. It thus cannot be excluded that control beliefs are as fundamental as socioeconomic grouping when it comes to affecting life-course pathways for people (e.g. see footnote 3 in 18). Simultaneously, in order to avoid psychologising of social problems, more research is needed to find out where the low control beliefs come from. Environmental factors, possibly other than socioeconomic, might interact with genetic factors in creating these individual differences (12). Further research could, for example, study how early life parental support, secure attachment and bonding might be important in the development of control beliefs in children and adolescents $(19,20)$. Further research should also examine how control beliefs could be more strongly embedded in a personalised approach of public health professionals working with youth (e.g. 21,22). Questions in need of an answer are, for example, how to detect low control beliefs, at what age should monitoring start, do gender and childhood diseases matter, how do low control beliefs relate to self-esteem, insecurity and emotional instability (particularly during puberty), and which interventions are available. As reported above, there is, however, first a need for cross-validation of the findings in prospective designs and for more stringent tests of the causal direction of the relevant mechanisms.

\section{Conclusion}

Independent of the socioeconomic background, low control beliefs are related to heightened odds of both poor health and school dropout. Individual differences in control beliefs might thus be as fundamental as socioeconomic conditions in generating life-course socioeconomic and health-related pathways. Although the findings should first be cross-validated in prospective studies, public health professionals working with youth might already start considering early interventions in youth with all too fatalistic and powerless mind-sets.

\section{Acknowledgements}

We thank the young adults who participated in the study for providing their valuable information. We also thank the many medical students who helped in collecting data and doing preliminary data cleaning, analyses, and reporting. 


\section{References}

1. Bobak M, Pikhart H, Rose R: Socioeconomic factors, material inequalities, and perceived control in selfrated health: cross-sectional data from seven post-communist countries. Soc Sci Med 2000, 51:13431350.

2. Bosma H: Socio-economic differences in health: are control beliefs fundamental mediators. In: Siegrist J, Marmot M, eds. Social inequalities in health; new evidence and policy implications. Oxford: Oxford University Press; 2006: 153-166.

3. Espinosa de Los Monteros K, Gallo LC: Fatalism and cardio-metabolic dysfunction in Mexican-American women. Int J Behav Med 2013, 20:487-94.

4. Savage M, Dumas A, Stuart SA: Fatalism and short-termism as cultural barriers to cardiac rehabilitation among underprivileged men. Sociol Health IIIn 2013, 35:1211-1226.

5. Skinner EA: A guide to constructs of control. J Pers Soc Psychol 1996, 71:549-570.

6. Wheaton B: The sociogenesis of psychological disorder: an attributional theory. J Health Soc Behav 1980, 21:100-1024.

7. Bosma H, van de Mheen HD, Mackenbach JP: Social class in childhood and general health in adulthood: questionnaire study of contribution of psychological attributes. Brit Med J 1999, 318:18-22.

8. Bosma H, Marmot MG, Hemingway H: Low job control and risk of coronary heart disease in Whitehall II (prospective cohort) study. Brit Med J 1997, 314:558-565.

9. Schooler C, Kohn ML: Job Conditions and personality: A longitudinal assessment of their reciprocal effects. Am J Sociol 1982, 87:1257-1286.

10. Lachman ME, Weaver SL: Sociodemographic variations in the sense of control by domain: findings from the MacArthur studies of midlife. Psychol Aging 1998, 13:553-562.

11. Wang LY, Kick E, Fraser J, Burns TJ: Status attainment in America: the roles of locus of control and selfesteem in educational and occupational outcomes. Sociol Spectrum 1999, 19:281-298.

12. Mackenbach JP: Genetics and health inequalities: hypotheses and controversies. J Epidemiol Commun $\mathrm{H}$ 2005, 59:268-273.

13. Theunissen MJ, van Griensven I, Verdonk P: The early identification of risk factors on the pathway to school dropout in the SIODO study: a sequential mixed-methods study. BMC Public Health 2012, 12:1033.

14. Elich M, Sinnema G: Chronische ziekten en lichamelijke handicap. In: Bakker GA, Van der Zeben DMCB, Dewispelaere AJ, eds. Handboek kinderen en adolescenten. Houten: Bohn, Stafleu, Van Loghum; 2010:75-95. [in Dutch]

15. Van de Mheen $\mathrm{H}$ : Inequalities in health to be continued? A life-course perspective on socio-economic inequalities in health. Wageningen: Ponsen \& Looijen; 1998.

16. Pearlin I, Schooler C: The structure of coping. J Health Soc Behav 1978, 19:2-21.

17. Sherer M, Maddux JE, Mercandante B: The self-efficacy scale: construction and validation. Psychol Rep 1982, 51:663-671.

18. Link BG, Phelan J: Social Conditions as fundamental causes of disease. J Health Soc Behav 1995, 35(extra issue):80-94.

19. Ramsdal G, Gjærum RG, Wynn R: Dropout and early unemployment. Int J Educ Res 2013, 62:75-86.

20. Skinner EA: The origins of young children's perceived control: mother contingent and sensitive behaviour. Intern J Behav Developm 1986, 9;359-382.

21. Syrina EV: Integrating personalised perspectives into Child and Youth Health Care: A long and winding road? Maastricht: Maastricht University; 2014.

22. Lupien SJ, Ouellet-Morin I, Trépanier L: The DeStress for Success Program: effects of a stress education program on cortisol levels and depressive symptomatology in adolescents making the transition to high school. Neurosci 2013, 249:74-87. 


\section{Appendix}

\section{Appendix 1}

Unstandardised regression coefficients (95\% confidence intervals) of current health status by recalled low control beliefs, adjusted for age, sex (model 1), and additionally adjusted for family composition, and ethnic and socioeconomic background (model 2) in case and control group, separately. ${ }^{a}$

\begin{tabular}{lllll}
\hline & $\begin{array}{l}\text { Cases } \\
\text { Model 1 }\end{array}$ & $\begin{array}{l}\text { Cases } \\
\text { Model 2 }\end{array}$ & $\begin{array}{l}\text { Controls } \\
\text { Model 1 }\end{array}$ & $\begin{array}{l}\text { Controls } \\
\text { Model 2 }\end{array}$ \\
\hline Mastery & $0.89(0.67-1.12)$ & $0.83(0.60-1.07)$ & $0.61(0.44-0.78)$ & $0.58(0.41-0.76)$ \\
Self-efficacy & $0.82(0.57-1.07)$ & $0.78(0.52-1.03)$ & $0.58(0.36-0.79)$ & $0.57(0.35-0.78)$ \\
\hline
\end{tabular}

${ }^{a}$ Ordinary least squares regression analyses using continuous scores of current health status (ranging from 1 : poor health to 10: excellent health), mastery (ranging from 1: low mastery to 5: high mastery) self-efficacy (ranging from 1: low self-efficacy to 5: high self-efficacy), and socioeconomic background (continuous score, being the mean of standardised education and deprivation variables).

\section{Appendix 2}

Participation (in terms of returning questionnaires) according to case-control status, sex, age, and mean house price in postcode of respondent.

\begin{tabular}{llll}
\hline & $\begin{array}{l}\text { Non-participation } \\
(\mathrm{n}=7581)\end{array}$ & $\begin{array}{l}\text { Participation } \\
(\mathrm{n}=1049)\end{array}$ & p-value \\
\hline $\begin{array}{l}\text { Case-control status (\%) } \\
\text { Controls }\end{array}$ & 92.9 & 7.1 & $<0.001$ \\
$\quad$ Cases/dropouts & 79.6 & 20.4 & \\
Sex (\%) & & & $<0.001$ \\
Boys & 91.3 & 8.7 & \\
Girls & 83.7 & 16.3 & \\
Age (mean) & 19.25 & 19.09 & $<0.001$ \\
Mean house price (mean) & 145690 & 158380 & $<0.001$ \\
\hline
\end{tabular}





\section{Chapter 6}

\section{"I think that the whole family needed help, but they only focused on me"}

An interview study with young adults about their childhood and dropping out of school

Theunissen M-J, Verdonk P, Bosma H, Feron F. "I think that the whole family needed help, but they only focused on me" An interview study with young adults about their childhood and dropping out of school (submitted for publication). 


\section{Abstract}

\section{Background}

School dropout is a complex public health problem. From the perspective of young adults, we aimed to gain an in-depth understanding into the processes that lead to school dropout.

\section{Methods}

We conducted 25 semi-structured interviews, guided by a topic list based on previous case-control studies and the literature, with young adults (22-25 years), who dropped out of school or graduated with a delay. Transcripts were analyzed thematically.

\section{Results}

Being excluded from relevant social groups, sometimes even violently, had major consequences for the interviewees' self-esteem and in turn self-esteem appeared to determine whether someone is included or not. Social problems tended to be individualized and self-efficacy and support seemed to be the sources for resilience.

\section{Conclusion}

This study contributes to a better understanding of the underlying causes in the processes towards school dropout. Public health professionals can play an important role in improving students' self-esteem and control beliefs. When problems occur, the whole system (beyond the individual) should be included in the solution. 


\section{Background}

Dropping out of school can have a huge impact on the lives of those affected. It is associated with poor mental and physical health, an unhealthy lifestyle and deviant behavior, such as delinquency and drug use (1-3). Furthermore, early school leaving reduces the chances of finding a job and is strongly linked to social exclusion, both as a cause and as a consequence $(4,5)$. The issue of school dropout is also closely linked to the broader issue of socioeconomic health differences, characterized by people in lower socioeconomic positions dying at younger ages and, within their shorter lives, having a higher prevalence of many health problems (6).

There is a need for an in-depth understanding of the processes that lead to school dropout from the perspective of young adults. Such understanding will contribute to the development of interventions that aim to prevent school dropout, thereby meeting the needs of the youngsters involved. Theoretical and empirical findings indicate that the pathways to school dropout often begin early in life and represent a complex and gradual process that may differ for boys and girls from different socioeconomic groups (7-9). General deviance theory suggests that students who are involved in drug and alcohol abuse, or other deviant behavior are more likely to drop out (1). Similarly, deviant affiliation theory indicates that peers' beliefs about school and academic achievement influence their friends' behavior and attitudes (10). The transactional model of development (11) suggests that children with early behavior problems are at risk for developing academic problems and experiencing rejection from their prosocial peers. Their lack of prosocial connections can lead them to form connections with deviant peers and in turn engage in other delinquent acts. Further, family socialization theory posits that the students' home environment affects their academic achievement in the classroom (1). Stressful events such as parental divorce, family conflict, and loss of a loved one can also influence how a student behaves in and outside the classroom (12). In addition, parents' education level and the importance parents attach to academic achievement can affect a student's sense of purpose and agency in the school environment. Finally, cumulative risk theory predicts an inverse association between the number of risk factors and positive adjustment to life events $(13,14)$. In addition, the number of risk factors during childhood and adolescence is related to the number of negative outcomes, including school dropout (15).

Many risk factors have been identified that influence school dropout (3), but less is known about the perceptions of the youngsters themselves. Qualitative research may identify contexts and relationships that have been overlooked to date and enrich the array of empirical findings. There is a clear need for triangulation, by using both participants' own perceptions of, and reasons for dropping out of school and findings from quantitative studies $(16,17)$. Hence, in the current study, we listen to the voice of the youngsters themselves to address the following question: "How do young adults who graduated with a delay or dropped out of school, perceive their life history and particularly their time in school?" 


\section{Methods}

\section{Setting and participants}

This study is part of the Stay in or Drop out (SIODO) study, a sequential mixed methods study where a quantitative case-control study with questionnaires and information from the youth health care files is followed by and input for a qualitative study with interviews and subsequent focus groups (18). This paper focuses on the interviews. We selected young adults, who consented to being approached for an interview, from the questionnaire responders in the previous quantitative case-control study (both dropouts and delayed graduates). The municipal compulsory education department of the city of Eindhoven, the Netherlands, selected all young adults aged 18-23 years who had not yet met the Dutch minimum educational requirement for an adequate start in today's labor market at the start of the 2010-11 school year. At this age, most students should already have obtained the required 'basic qualification' (which is equivalent to higher general secondary education, pre-university education or intermediate vocational education, Level 2) $(19,20)$. A proportion of these young adults remained in school during the 2010-11 school year to obtain this qualification, while others dropped out of school during that year without qualification (the school dropouts).

Purposive sampling seeks to maximize the depth and richness of the data and increases the validity of the results. Therefore, we grounded our purposive sampling in our previous findings $(17,21,22)$ and the literature. We used an intracategorical intersectional approach to study the phenomenon of early school leaving, by searching within the group of school dropouts and delayed graduates (intracategorical) for the most relevant social categories (socioeconomic status (SES), gender, ethnicity) (23). Regarding risk factors for school dropout, we therefore invited young men and women from different backgrounds such as high/low SES, Western/ non-Western ethnicity and also with a good or bad self-reported health, highly masculine or feminine self-scores and with different educational levels (Table 1).

The selected young adults received an email informing them about the interview. One reminder was sent after a week. Young adults who consented to participate in the interview were called to make an appointment or emailed with information about date, time and place. Two days before the interview took place, participants received a reminder by email. We approached 47 young men and 40 young women who dropped out of school, of whom 7 (14.9\%) and 11 (27.5\%) participated respectively. To validate the themes, we also approached 8 young men and 20 young women who graduated with a delay. Four men (50.0\%) and 3 women (15.0\%) participated. Participants received travel expenses and a €15, - cinema or gift voucher for their participation. The interviewer did not know the interviewees. 


\section{Ethics statement}

Approval for conducting this study was granted by the Medical Ethics Committee of Maastricht University (METC 11-4-099, decision 22 August 2011).

\section{Interviews}

To gain an in-depth understanding of the meaning of the previously found determinants and to contextualize school dropout as a phenomenon within the boys' and girls' lives from their own perspectives, we needed to hear the story of the young adults themselves. We expected that marginally or less acceptable views and sensitive issues were best assessed in individual interviews (24). The interviews took place from September to December 2014, in a public health service (20), at home (3) or at work (2), according to the wishes of the interviewee and lasted 45-90 minutes. We pilot tested an interview with a 22 year old man, who had dropped out of tertiairy education and thus did not participate in our study (he had already attained a basic qualification), and adjusted the topic list. An experienced Dutch white female youth health care physician (first author) performed individual face-to-face semi-structured in-depth interviews. A friend/relative of the interviewee accompanied participants 7 and 15 . We used open-ended questions to gain an in-depth insight based on personal experiences. The semi-structured interviews were iterative and guided by a topic list based on our previous studies $(17,21,22)$ and the literature (Table 2). The topic list has been adjusted over time, for example the topic "feel that you belong to" is added after the first interview. The interviewer applied little structure, which allowed participants to tell their story the way they wanted. Furthermore, for the second part of the interview we showed 22 cards with pictures of previously identified determinants of school dropout (17). This made it easier for the participants to stay focused and to control the topics they wanted to talk about. 


\section{CHAPTER 6}

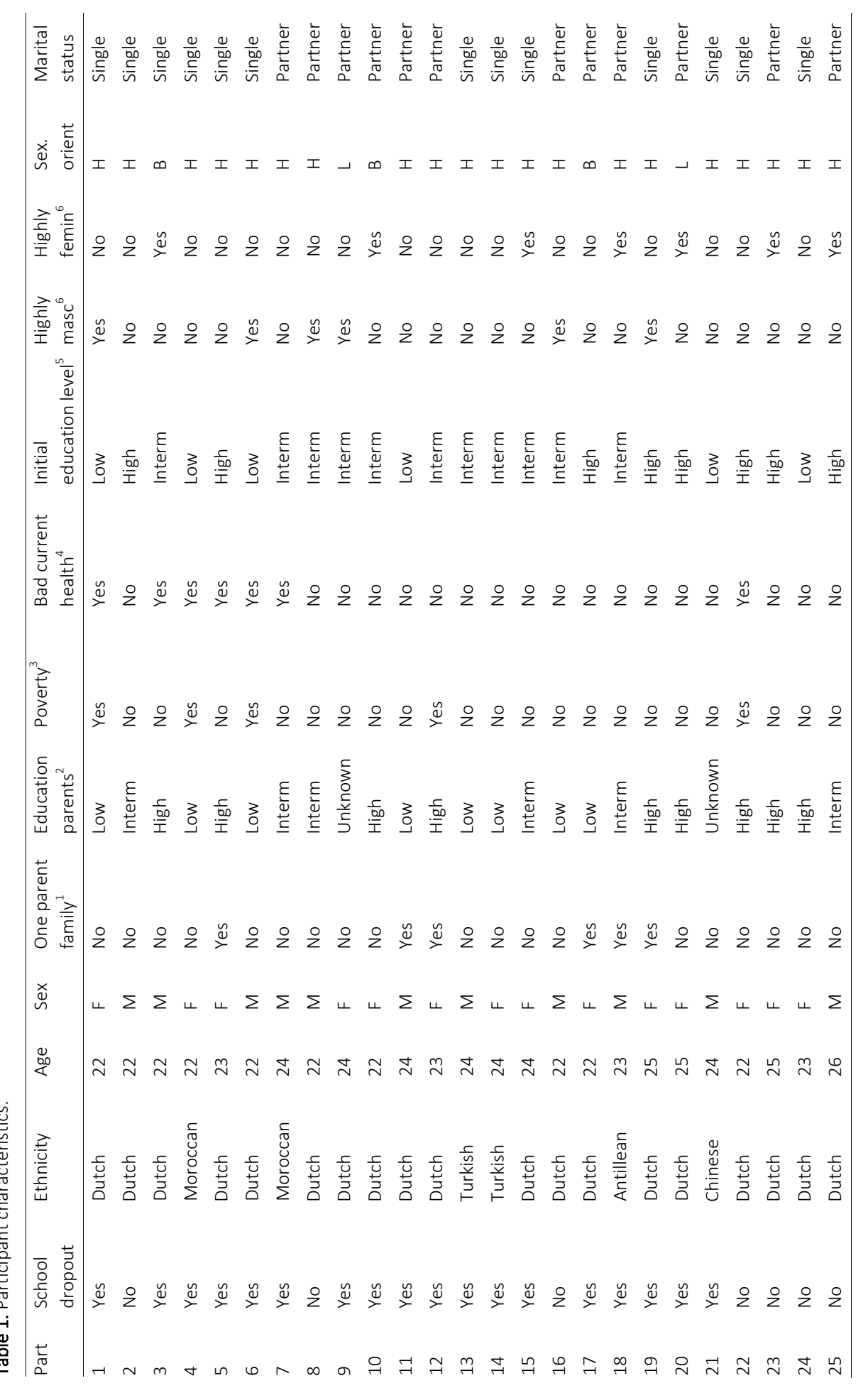


Part=participant number, $F=$ female, $M=$ male, Interm=intermediate, masc=gen, femin=feminine, Sex. orient:=sexual orientation, $\mathrm{H}=$ heterosexual, $\mathrm{L}=$ lesbian, $\mathrm{B}=$ bisexual. ${ }^{1}$ Living in a one-parent family at the time that they attended primary school. ${ }^{2}$ The highest education completed; low (no education, primary education, lower vocational education or pre-vocational theoretical program), intermediate (secondary vocational education, higher general secondary education or pre-university education) and high (higher professional education or university education). ${ }^{3}$ Based on four questions on material and social deprivation when at a primary school and at secondary school (each with four categories; never, sometimes, regularly, always). Poverty refers to having not enough money for food, clothes, or participation at sports or school activities, regularly or always. ${ }^{4}$ Based on self-rated current health (varying from 1: not healthy at all to 10: very healthy). Bad current health refers to a score lower than 7. Initial education level at the entry of basic secondary education; low (vocational pathways of a preparatory vocational secondary education), intermediate (theoretical learning pathway of a preparatory vocational secondary education) and high (senior general secondary education or pre-university education). ${ }^{6}$ Based on self-rated masculinity/femininity (varying from 1: not masculine/feminine at all to 10: very masculine/ feminine). The question "how feminine/masculine do you think you were at the age of 16" was exemplified by "acting as a leader, ambitious, dominant, independent, aggressive, tough behavior" as masculine traits and "helpful, sensitive, shy, caring, understanding, friendly" as feminine traits . Highly masculine/feminine refers to a score above 8.

Table 2. Interview topic list

\begin{tabular}{ll}
\hline General introduction & $\begin{array}{l}\text { Information, informed consent, approval for recording, confidentiality, duration, } \\
\text { demographics } \\
\text { First question }\end{array}$ \\
Family & "Can you describe a normal day?" \\
& divorce, domestic violence, support from parents, socio economic status (enough \\
& money for school trips, a car, a computer, new clothes) \\
& Relation with teachers, transition to another school, Coping with tests and pressure \\
School & to perform, support, decision to stop with school, reaction from environment on \\
& decision to stop with school. \\
& Friends, relationships, bullying, to belong to, sexual orientation, peers who stopped \\
Peers & with school, support \\
Health in childhood, school absenteeism, reason to stop with school & Sports, part-time job \\
Participation & $\begin{array}{l}\text { Tough girls, tough boys, caring boys, gender roles, homosexuality, one parent } \\
\text { Tamily, relation between parents, poverty, young parents, swimming lessons, motor } \\
\text { development, hospital admission or surgery, a younger brother or sister, school } \\
\text { absenteeism, part-time job, learning difficulties, disease, hearing problems, } \\
\text { externalizing behavior, internalizing behavior, smoking, alcohol or drug use control } \\
\text { beliefs, self-confidence, }\end{array}$ \\
&
\end{tabular}

First, the interviewer introduced the study and informed consents were signed. Then, we started the interview with the open question: "Can you describe a regular day?" Subsequently we asked each participant "Can you describe your childhood and your time at school?" The first part of the interview the participants told their life story about their childhood, their family, leisure activities, school, and their relations with family, teachers and peers. In the second part of the interview, participants chose some cards with topics they especially wanted to talk about. Cards with topics already discussed in 
the first part of the interview were removed. We emphasized that we were interested in hearing their personal opinion and personal experiences. The transcription of the interviews was sent to the participants for approval and further comments (member check). All participants approved the transcription and none of them had any comments. Interviews were held until data and theoretical saturation occurred and no new topics came up in the interviews and no new ideas emerged from the data during analysis $(24,25)$. Furthermore, variation within the emerged themes was described, relationships between the themes established and subsequently validated by following interviews.

\section{Data analysis}

All interviews were tape-recorded and transcribed verbatim and notes were taken. All authors read notes and transcripts to gain a sense of the depth of the data and to collect and discuss ideas. We all wrote memos of the interviews. We used a grounded theory approach (26) and interim analyses were conducted by starting the analytical process during data collection, which allowed us to go back and refine questions, develop hypotheses and explore these in more depth. Furthermore, we have read the data to identify emergent as well as anticipated themes and categories.

The analyses were done using Microsoft Excel. First, MT and PV coded two interviews separately (open coding) and designed the initial code tree. The code tree was discussed with all authors. Then HB and FF individually coded two different interviews using the code tree. MT also coded these interviews. All authors discussed the differences and difficulties in coding and clarified and negotiated until consensus was reached. In general, there was a high level of agreement and little or no changes in the code tree was necessary. Subsequently, MT coded all remaining interviews (Table 3).

The transcripts were analyzed thematically to identify and report patterns and categories in the data. After clustering the data into overarching groups, MT and PV constructed themes by combining codes to overarching categories (axial coding). Finally, the core categories emerged (selective coding), which best explained pathways to school dropout (27). Discussion between all authors led to three major themes to construct a thematic framework. 
YOUNG ADULTS ABOUT THEIR CHILDHOOD

Table 3. The coding tree

\begin{tabular}{|c|c|c|}
\hline Codes & Subthemes & Themes \\
\hline $\begin{array}{l}\text { Violence } \\
\text { Bullying }\end{array}$ & $\begin{array}{l}\text { Feeling (un)safe } \\
\text { Experiencing violence } \\
\text { Anxiety } \\
\text { Lack of a stability }\end{array}$ & Safety and support \\
\hline $\begin{array}{l}\text { Participation in school, work, leisure } \\
\text { activities, with peers, family }\end{array}$ & Participation & \\
\hline $\begin{array}{l}\text { Family relations } \\
\text { Peer relations } \\
\text { Intimate relations } \\
\text { Relation with teachers } \\
\text { Relation with employers } \\
\text { To belong to } \\
\text { understanding }\end{array}$ & $\begin{array}{l}\text { Trust } \\
\text { Motivated/stimulated } \\
\text { Feeling connected } \\
\text { Being understood }\end{array}$ & \\
\hline $\begin{array}{l}\text { Physical complaints } \\
\text { Mental complaints } \\
\text { Conduct disorders } \\
\text { Learning disabilities } \\
\text { Material circumstances }\end{array}$ & $\begin{array}{l}\text { Limitations } \\
\text { Possibilities }\end{array}$ & $\begin{array}{l}\text { Expectations and } \\
\text { possibilities }\end{array}$ \\
\hline $\begin{array}{l}\text { Emotions } \\
\text { Clearness } \\
\text { Acceptance }\end{array}$ & $\begin{array}{l}\text { Expectations to } \\
\text { yourself, to others and } \\
\text { from others } \\
\text { In/exclusion } \\
\text { Norms and rules } \\
\text { Future perspectives } \\
\text { Aims }\end{array}$ & \\
\hline $\begin{array}{l}\text { Being yourself } \\
\text { Self-confidence } \\
\text { Performance driven } \\
\text { Gender } \\
\text { Sexual orientation } \\
\text { Language } \\
\text { Student actions } \\
\text { Substance use }\end{array}$ & $\begin{array}{l}\text { Self-confidence } \\
\text { Control beliefs } \\
\text { Coping strategies }\end{array}$ & \\
\hline $\begin{array}{l}\text { Interventions inside school } \\
\text { Interventions outside } \\
\text { school }\end{array}$ & Focus on the individual & $\begin{array}{l}\text { The individual } \\
\text { and beyond }\end{array}$ \\
\hline
\end{tabular}

\section{Validity}

In order to prevent bias we used data triangulation by performing a member check and taking field notes during the interviews. A Dutch summary of the main findings was sent to 24 participants, who earlier indicated to be interested to participate in a member check. Eighteen participants responded. Twelve of them left school early; they all recognised themselves in the findings. From the five participants who graduated with a 
delay, three recognised themselves in the summary, whereas two mentioned that the findings did not apply to them; they both had pleasant memories about their childhood. Nine participants provided additional remarks which were incorporated in the results that clarified and strengthened our findings. Findings from the previous case-control studies, the literature, and our own experiences with this group of students informed our interview guide. We also applied researcher triangulation on analytic decisions by discussing the steps with all authors until we reached consensus. Each researcher provided his/her own perspectives. More specifically, MT and FF are youth health care physicians, PV is an occupational health psychologist with a gender studies background and $\mathrm{HB}$ is a social epidemiologist with a particular concern for socioeconomic differences. Two of the researchers are male and two are female. All authors have a Dutch Caucasian background.

\section{Results}

Three themes emerged about how young adults, who graduated with a delay or dropped out of school perceived their life history and particularly their time in school: (1) safety and support, (2) expectations and possibilities, and (3) the individual and beyond.

\section{Theme 1. Safety and support.}

Interviewees experienced a lot of violence in different situations and in different ways, which decreased their self-esteem. They reported physical, psychological and/or sexual abuse by parents, other relatives or siblings. Furthermore, several interviewees reported feeling humiliated, discriminated or unfairly treated by teachers and employers. Having a mentally ill or addicted parent created an unsafe environment, as youngsters had to be constantly aware of their parent's unpredictable behavior. Reported fights were mostly about financial problems. Several interviewees mentioned that they were frightened of their parents getting a divorce when they verbally or physically fought with each other, and endured the fights hoping their parents would stay together:

"I was afraid of my parents getting a divorce that was all I could think about. Verbally fighting may happen, as long as they do not, uh, well, hitting each other is just acceptable, depending on the situation" (R21, male, left school early)

However, for some youngsters their parents' divorce was also a relief because the domestic violence, the abuse or the humiliation ended, although youngsters often concealed being abused until later in life. For others, the parental fighting continued after the divorce and additional problems such as a loyalty conflict, financial hardships and losing their home occurred. Some parents used their children as a carrier pigeon or sent them to their ex-partner or a relative when they felt the child exhibited behavioral prob- 
lems, which made them feel worthless and insecure. Youngsters felt hopeless when teachers neither listened to their problems nor responded to them, which especially happened in case of family problems. However, some interviewees found warmth and support at a friend's family or at work, which empowered them. Youngsters longed for security, and seemed vulnerable for being exploited, such as in the case of a female interviewee who took care of her stepfather by cooking and cleaning for him. However, although she considered him her dad, he kicked her out of the house when she got ill:

"And when he went to work, I made sure that his sandwiches were made, his shoes were polished and his clothes ironed. But then I fell ill. I stayed in bed with a thirty-nine degree fever, and I could not do anything. My stepfather did not mind for two days, but on the third day, he could not take it anymore. And he became angry with me. He told me he was done with me being sick, and we had a big fight about it. And eventually he grabbed my head and my ass and threw me out on the street in my pajamas. (R12, female, left school early)

Interviewees also mentioned being excluded, and experiencing mental and physical violence by peers, beginning early in primary school. An interviewee reported being bullied from the very first day at school and although she felt miserable about it, she thought this was how it should be. Being bullied and excluded decreased the youngsters' self-esteem, and made them feel unsafe at school, especially during breaks. Some youngsters kept the problems to themselves, afraid that the bullying further increased, or they reported themselves sick from school to avoid bullying classmates.

For me, the breaks were the worst of all [.....], I was always being bullied then, because I was shy and kind of a nerd, and I did not take any risks. Until, at a certain point, I went to school for the first, the second and third lesson but went home during the break. Quite frankly, I was just skipping classes [.....] until, at a certain point, uh, school called my mother uh, to tell her that I was suspiciously often ill at home, or to an appointment with my physician or dentist" (R12, female, left school early).

Others asked for help, but did not feel acknowledged by their teachers. However, some interviewees who repeatedly consulted their mentor reported that the bullying stopped at a certain moment. They needed to feel secure with their teacher before being able to discuss their problems.

"yes I was being bullied and I was fed up with it, I thought, the hell with it, I am going home. [.....] Uh, well, I never really talked about that. As I already said, I started to annoy the teacher or I just went home. So I did not really feel connected to my teachers, because I was a nuisance in the classroom. I did not really eh, have a bond with those teachers, so I did not think I needed to discuss it" (R25, male, stayed in school). 
Interviewees felt most understood by someone with similar experiences, in a course with other youngsters who experienced depressive symptoms or when their psychologist mentioned having a history of drug abuse him or herself. Interviewees who received support that corresponded to their needs and expectations felt more motivated to continue or start an education. However, youngsters were used to accommodate to other people's needs, for instance when their parents had never complimented them, but rather always got angry about disappointing school-grades or when something else went wrong. A male interviewee mentioned that he started a course so that his social worker would be proud of him. According to this youngster, unlike his parents, his social worker always believed in him.

"Eh, a youth worker told me, I was naturally gifted to become a youth worker. To me, that was the turning point. I knew that I really had to go for it. I also said, "from now on I will do anything to succeed." I followed a course for city trainer. [..... At my graduation, I called the youth worker, that guy was always there for me. I told him to meet me, because I had a nice present for him. [.....]. I told him that I had done the City Training course, so I could help organize things. And, um, he reacted very positive. And that's why I also told him that I would do anything to see if I could go to school again, if it is financially possible."(R11, male, left school early).

\section{Theme 2. Expectations and possibilities}

Bullied, humiliated and abused interviewees not only mentioned how these events negatively affected their life, self-confidence, and self-esteem, but also how that manifested in self-harm, like auto-mutilation, anorexia and substance abuse. Interviewees not only needed someone who believed in them, but also needed to believe in themselves.

"Eh, well it depends on how the feedback was given, but eh I find it difficult to be criticized because I eh, if I get the feeling that I am doing something wrong, it's the same feeling my mother used to give me, that I am doing something wrong and it still really hurts badly. [.....] I am not looking forward to going to school because I am afraid that I am too stupid to learn anything. And I am afraid of the class, of the other girls. But I will be very happy to finish my education. Then I can finally be proud of myself, because I will have accomplished something" (R 10, female, left school early)

Acceptance by peers appeared to be very important and many interviewees mentioned that they tried almost everything to fit in; they changed their looks, started smoking and exhibited disruptive behavior in the classroom, to look like a cool kid. However, when that was not enough, they looked for another group to join, such as some masculine female interviewees, who told us that they joined a hangout where tough behavior was 
accepted. Being accepted in a group is about in- and exclusion, rejection, recognition and recognizing, and being able to be 'yourself'. It is about the youngsters' sense of who they are and what they are capable of, and of what they find important in their lives. A particular issue of importance seemed to be a shared group identity, which may include religion or other shared characteristics that are important to them. Group members are expected to accept the applicable norms and rules of that group such as a male interviewee who mentioned that he was rather soft, but exhibited stereotypical masculine behavior, because that was expected in the boys' school he visited. However, some rejected being part of particular groups, and the inclusion in certain groups coincided with strong exclusion from other groups. Physical and mental limitations might cause exclusion and an interviewee mentioned that being labeled as a psychiatric patient alienated people, which eventually made her lose herself. Being excluded from any social group decreases one's self-esteem and in turn, self-esteem and being selfconfident appeared to determine whether someone is included or not. A shy female interviewee reported being bullied for wearing baggy trousers from the men's' department, while a confident male interviewee was accepted by his peers wearing female skinny jeans, yet to a certain extent:

"Then I dyed my hair black, I had piercings and then eh, it looked like an Emo style. Well, I think that look is very awesome, so I also wanted to create that image. But I had to wear skinny jeans, which were not available in men's sizes at the time, so I just walked around in girl's jeans. And I uh had built up such a status, that it was simply accepted. [.....] A lot of guys looked very strange to me though and a lot of people acted stupidly. Lots of guys called me names, like bitch. [.....] At my graduation, I wore uh pink skinny jeans, and was therefore not allowed in the men's room. Other guys told me; you are not a man, piss off' (R3, male, left school early)

Expectations are not only about reciprocal expectations in relationships, but also about current and future perspectives and finding oneself capable of reaching them, and about understanding existing norms and rules and willingness to confirm to them. Having job prospects motivates youngsters to go to school, but an initial life goal can be disrupted. Some interviewees reported that medical and social problems made it hard to perform in a certain job, and one interviewee mentioned that he lost his motivation because he was rejected for an internship. According to the interviewed young adults, teachers responded differently to students with learning difficulties. Some youngsters received the expected extra support, but others felt misunderstood or felt bullied or humiliated by their teachers. Interviewees with learning difficulties often lag behind at school, repeated a grade, or continued the education at a lower level or at another school when they could not meet the requirements. This would further decrease their self-esteem. Some interviewees mentioned that they missed the clarity and structure in their education because during their studies, no textbooks were used. Some other 
young adults reported feeling insecure because they were unfamiliar with schools' requirements and expectations. For some interviewees, however, demands and expectations caused stress because they felt pressured to perform. A female interviewee mentioned to feel stressed and pressured because she did not match the school system. She had to join a compulsory internship at a day-care centre, which contradicted to her principles of child raising. She believed that a child should be with his or her parents. The transition from primary to secondary school coincided with positive and negative expectations which varied from making new friends and a hope for a fresh start to fear of being bullied and not being able to perform well. Non-western interviewees expected better quality of education at a so-called 'white school' with a white majority student population, and they expected a higher peer status when they played or connected with white Dutch children. However, cultural differences sometimes lead to misunderstandings, such as this interviewee who felt insecure when she was told to go home before the family dinner, while she was expecting and accustomed to be invited:

"At the time, in primary school I befriended a Dutch girl and uh came to her house where, [.....] they told me they were having dinner at five o'clock, and was I being picked up at five? Then I thought, okay, then I make sure I will be picked up at five. With us it is different. We say tell your mom or dad that you can be picked up at six o'clock because you will eat with us first. With Dutch families it is different. And uh, I thought it was very weird. [...]So yes, when she came to our house, she was invited to have dinner with us. And then I thought, why is she invited when I am not invited to eat at her place. I thought that was very typical"(R14, female, left school early)

Parents have expectations towards their children's future regarding health, education, gendered behavior and sexual orientation, and when children do not live up to them they may be disappointed. Some young adults reported that their parents expected them to take a parental role in caring and housekeeping. Interviewees, feeling responsible for the wellbeing of their parents and siblings complied that request. This especially applied to mentally ill parents, where youngsters were afraid that their parents would hurt themselves but also to parents who did not understand the Dutch language.

It happened the year my parents got a divorce. It was very difficult at the beginning. On the other hand, I was very excited to go to secondary school. So it was a mixed feeling actually. One day, I was looking forward to going to school, the next day, I really did not want to go to school because I had to wake up my little brother and sister. My younger brother had to go to school in Eindhoven by bus. And I had to make sure that my little sister walked to her school by herself. They both had to get out of bed in time. So actually, I felt more like a parent at that time than I was being myself."(R11, male, left school early) 
Rather, young adults expected their parents to support them and they felt left in the lurch when they had to remain silent about their mental problems or their sexual orientation. However, interviewees, who reported being accepted for who they are by family and peers, which made them self-confident, had pleasant memories about their childhood and their time in school.

\section{Theme 3. The individual and beyond}

The individual and beyond refers to how parents, teachers and health professionals focus on the individual youngster despite their being part of a much wider system. Members of a particular system constantly influence each other's behavior, and a youngster's behavior does not only depend on personal characteristics, but also on the behavior of their environment such as family, peers and school. For example, interviewees mentioned automatically being involved in their parents' fight, even when parents tried to leave them out, and reported being bullied for not participating in disruptive behavior such as joining their classmates in cheating tests and calling the teacher names. Many of the interviewees consulted several psychologists, psychiatrists or followed courses to train social skills. However, according to the interviewees, individual psychological help often was insufficient if the environment was not incorporated in the necessary changes. Moreover, it could decrease self-esteem such as in an interviewee who mentioned that she was always searching for parental approval but never received it, which made her try even harder and eventually let her fail. This interviewee also mentioned having low self-esteem and blaming herself for the problems she experienced, because her parents had called her a problematic child and blamed her for all the family problems:

"That's a tough one. I think that the whole family needed help, but they only focused on me. I took some courses to improve my social skills. However, if it is not appreciated, then it is worthless. Then you relapse into your old patterns, so eh, you can say that I have not remembered much of what I learned."(R20, female, left school early)

Since you cannot change a system on your own, some interviewees tried to escape the system by running away from home or playing school truancy, by seeking a safe place at the skate track, or by withdrawing socially. Especially when interviewees experienced stress in the family, they mentioned that they shut themselves off and wanted to get out of the situation. Some young adults mentioned quitting school to earn money so that they could live on their own, away from family problems. Other interviewees escaped into alcohol or drug use or extensive gaming, or they eliminated one of the problematic systems by quitting school.

However, some interviewees got motivated by the bad circumstances at home or at work, and took control to improve their own situation by education. Others managed to 
withdraw from the negative effects of their environment because they experienced enough self-esteem and felt powerful enough to stand up against a dominant father or to be a role model or guardian for a sibling:

"The last two years of my intermediate vocational education (MBO), my sister started her first year at the same school. And uh, then I thought something like oh I might better stay in school. Because she, yes she knew I was in school a certain period, so then I thought something like oh I'll stay that extra hour because I know that my sister is here. Because um yeah, I always wanted to be some kind of a role model for her" (R24, female, stayed in school)

Figure 1 shows the thematic framework, based on the young adults' stories.

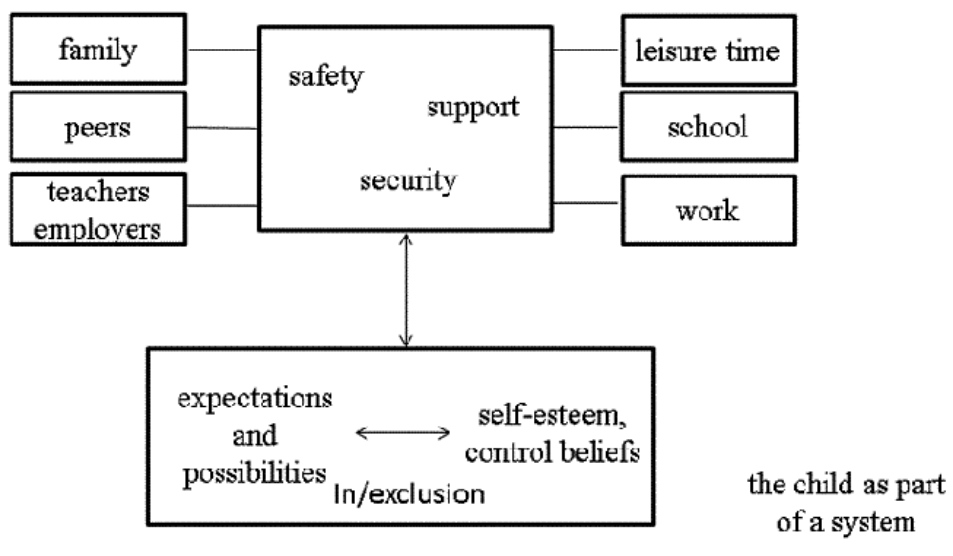

Figure 1. Thematic framework to understand the process of dropping out of school

Students imminent for school dropout face several problems that must be considered within their context. In order to productively cope with their problems and finish their education, students need resources such as self-esteem, self-efficacy and social support. Lacking safety and social support or being excluded from relevant social groups, may decrease youngsters' (future) expectations and has major consequences for their selfesteem and control beliefs. In turn, self-esteem appears to determine whether someone is included or not. Physical, cognitive and mental limitations or not conforming to expected group rules and norms may exclude a youngster from social groups. The expectations of oneself and of the environment about possibilities and limitations interact with one's self-image and are influenced by the way the youngsters' problems are approached by others. Therefore, focusing on the individual without incorporating the wider context in addressing problems may further decrease a youngster's self-esteem.

In the following story of a female interviewee, all themes are clearly present. She never felt accepted in secondary school neither by her classmates, nor by her teachers. After she repeated the first grade, she connected to a group of peers with disrupted behavior and started to smoke marihuana, but she still never really felt accepted. Eventually, these peers bullied and even threatened her. In the quote below, she talks about her druguse and what made her decide to quit school: 
"Not to experience pain, feelings and emotions. Uh even feeling good, yes I did experience that from smoking marihuana, but especially not feeling anything, not having to deal with eh how badly I was actually feeling. Not having to deal with the fact that my sister is autistic and eh lives in a closed institution, not having to deal with the fact that my father calls me every week to say that he is going to commit suicide and then leaves me home alone. For not having to deal with those situations, I used drugs [.....] It became all a bit too much for me. My relationship broke up. At home, it was not going great. I was not feeling very well and in that case, the easiest thing to get away from is school. I cannot get away from my father, I cannot leave my sister, I cannot get rid of the problems in my head, or my feelings, my abuse, my addiction because I simply was addicted. And the easiest solution then was to quit school" (R19, female, left school early)

\section{Discussion}

The aim of this study was to gain an in-depth understanding into the processes that lead to school dropout from the perspective of the young adults. Three themes emerged (1) safety and support, (2) expectations and possibilities, and (3) the individual and beyond. The findings in this study underline the theories mentioned in the introduction, which are about vulnerability, risk factors, and the association with school dropout. For example, some young adults, who dropped out of school were involved in alcohol or drug abuse (general deviance theory) (1) and family conflicts influenced interviewees wellbeing and school performance (family socialization theory) (1, 12). Interviewees needed resilience, obtained by control beliefs, self-confidence, and social support, to productively cope with their problems and finish their education. Lacking safety and social support or being excluded from relevant social groups, because of physical, cognitive and mental limitations, or not conforming to expected group rules decreased their (future) expectations and had negative consequences for their self-esteem and control beliefs. In turn, self-esteem appeared to determine whether someone was included or not. Focusing on the individual without incorporating the wider context in addressing problems also decreased interviewees' self-esteem. Some young adults, who graduated with a delay, felt accepted and experienced support from family, school and peers. They felt self-confident and had pleasant memories of their childhood and time in school.

The feeling of security in the child-parent relationship influences how youngsters experience relationships throughout their lives. Youngsters who are positively attached to their parents are more likely to like their teacher and feel positive about school, which in turn contributes to the connection to school (28). In this context, we may understand our previous case-control findings that children, who were having surgery or were hospitalized in the first years of primary school, were less likely to drop out of school. In such cases, parents possibly were more concerned and attentive (17). Fur- 
thermore, some interviewees who witnessed domestic violence or were suppressed or neglected by their parents did not feel supported by their teachers and lacked confidence in care. This is in line with findings that youngsters who experienced an authoritative parenting style at age 14 were less likely to drop out of school compared to those who perceived their parents as authoritarian, neglectful or indulgent (29). Being exposed in childhood to domestic violence between parents or from parent to child, is associated with psychological, emotional, social, behavioral and academic problems (30) and decreases a youngster's self-esteem $(31,32)$. Moreover, being exposed to different kinds of violence will also lead to reductions in feelings of mastery and perceptions of social support. In a former study, these youngsters felt more often powerless and believed that they were personally to blame for their victimization (32). Moreover, when they are victimized in different life domains, they miss any context in which they may gain a sense of personal efficacy (32).

Bandura's self-efficacy theory states that personal efficacy expectations determine whether coping behavior will be initiated, how much effort will be expended, and how long it will be sustained when experiencing obstacles (33). In our study, most interviewees who experienced parental divorce or domestic violence, tried to avoid the stressor by running away from home or from school, playing truant or dropping out of school. These stressors may be perceived as uncontrollable for some children which might be the reason that they choose to avoid them (34). In general, interviewees who stayed in school experienced a higher level of self-efficacy compared to interviewees who left school early.

A successful school career not only depends on grades, but maybe even more on how youngsters feel around peers and their belongingness to a social group (35). In line with the belongingness hypothesis (36) we found that the need to belong to peers seems to be a fundamental motivation. Interviewees would adjust their clothes, their behavior or even start smoking just to fit in. Other studies also found that substance use played a role in being socially integrated (37) but also seems to be a coping mechanism for youngsters who felt marginalized (38). In our study, some interviewees felt accepted neither by peers nor by teachers and experienced no belonging or connection to school. Such lack of connection can lead to poor grades, increased truancy, absenteeism and school dropout (39). Youth who are engaged with school feel more academically competent and are more connected to the institution. Moreover, they are more positively approached by their teachers and parents. Positive social relations can encourage youngsters to attend school, even those with learning difficulties (40) and can help youngsters to stay at school when there is an imminent risk of school dropout (41). Disengaged youth on the other hand, have academic difficulties, receive less positive support from teachers, and are more likely to associate with disengaged peers (42). Qualitative and quantitative studies report lack of social support by teachers as an important reason for dropping out of school $(40,43)$, which is in line with our findings. Some interviewees however, did not want to fit in the current educational system that 
moulds students to passive obedient citizens in a middle-class society and valued their own cultural norms.

In our study, interviewees reported that parents, teachers and health professionals focused on the individual youngster, while they actually are being part of a much wider system. According to Sameroff, problem behavior is a result of the way a person's characteristics affect and is affected by others (44). His unified theory of development views development as the result of a set of ongoing interactions between individuals, resulting in the change of each individual's behavior. From this view, the whole family and wider social system needs to be treated instead of focusing on the individual youngster with problem behaviors.

In the sampling we used an intracategorical intersectional approach to better understand differences in diversity and among others we invited young adults with different masculine and feminine self-images and with different ethnic and socio-economic backgrounds within the group of school dropouts and delayed graduates. Some interviewees reported not feeling accepted by peers or parents because they could not conform to culturally determined gender beliefs of how men and women should behave. In line with other studies, lonely masculine women with a low self-esteem reported to join deviant peers at a hangout $(45,46)$ and some male interviewees mentioned that they behaved in a stereotypical masculine way to be accepted by and belonging to their peers (47). However, striving for a masculine self-image is associated with antisocial behavior among boys and girls, which might increase the risk of dropping out of school $(8,21)$.

Interviewees with a non-Western background sometimes said that they experienced different norms and values at home and at school or at a friend's home. This could make them feel insecure and decrease their confidence. Non-Western youngsters mentioned the importance of going to a 'white' school and playing with Dutch children, but at the same time, they felt more attached to peers with the same ethnic background. These children negotiate between different cultural identities and it seems that not the compatibility of both cultures, but rather the feeling to belong to both cultural worlds determines a successful negotiation (48).

We expected a low SES to be directly related to school dropout, but the interviewees did not experience this as such. However, interviewees mentioned that rich peers joined each other and formed groups. They also reported that parental conflicts were mostly about financial problems and that there was not always enough money to join sports or to buy new clothes. The relation between SES and school dropout may be indirect, caused by a lower level of self-efficacy, since SES and self-efficacy are associated $(49,50)$. Noteworthy is also that non-response in SIODO was dominated by youngsters from lower socioeconomic neighbourhoods $(17,18)$. This may also explain why socioeconomic influences have been so difficult to detect in SIODO.

Dropping out of school is associated with unemployment, less income and health problems, which makes it a public health issue $(3,4)$. However, by considering school dropout to be the main problem, we fight symptoms rather than causes. Perhaps we 
should rather focus on the underlying causative factors and empower the children and their environments. The Dutch youth health care professionals offers free routine health examinations at set times, and in cooperation with parents and educational professionals, they can play a role in increasing students' self-esteem and control beliefs. It is important that they connect to children and youngsters, take their concerns seriously, and pay attention to warning signs of (risk of) physical and mental abuse. Moreover, entering a dialogue with students about their feelings of safety and belongingness to peers and family is important. Furthermore, they could ask what youngsters and parents really need and organize support correspondingly. When addressing students' problems, it is important that professionals consider the environment of the child/youngster. In forthcoming studies, we will discuss the themes found in this study, in homogenous focus groups with parents and with stakeholders from education and (youth) health care. We hope that the results will ultimately contribute to developing interventions that aim to prevent school dropout from a public health perspective.

\section{Methodological considerations and future research}

This qualitative study provides insight in the experiences of youngsters' lives and helps to understand and to contextualize the risk factors found in the large body of quantitative research about school dropout. We were able to use findings from the previous case-control study for the purposive sampling, which provided a lot of variation. We obtained many different views and experiences and data and theoretical saturation have been reached, as was confirmed by the member check and the youngsters' responses. Some limitations need to be mentioned. First, the study is retrospective and later experiences such as dropping out of school may modify the recall of earlier experiences of stress and the quality of relationships. Second, the interviews are conducted and analyzed in Dutch and the quotes are translated into English afterwards. Therefore, some nuances may be lost in the text (24). Third, the interviewer was an experienced youth health care physician, but not an experienced interviewer. However, she was aware of her different role and reflected on the interviews with PV. The interviewer did not know the interviewees. Furthermore, the interviewees were very open-hearted, even about very sensitive topics, such as sexual orientation, sexual and physical abuse, and addiction.

\section{Conclusion}

This study contributes to a better understanding of the underlying causes in the process towards school dropout. We found a great diversity in respondents' behaviors and in the complexity of their problems. However, they all longed for belongingness, safety, and support. Societal problems tended to be individualized and self-efficacy and support appeared sources of resilience in the face of hardships. The youth health care pro- 
fessionals can play a role in maintaining or even improving students' self-esteem and control beliefs. They could enter into a dialogue with students about their connections to peers and family. In the event of problems, the whole social system (beyond the individual) should be included in the guidance.

\section{Acknowledgements}

We wish to thank all participants of the interviews 


\section{References}

1. Battin-Pearson S, Newcomb MD, Abbott RD, Hill KG, Catalano RF, Hawkins JD. Predictors of early high school dropout: A test of five theories. journal of educational psychology. 2000;92(3):568-82.

2. Breslau J. Health in Childhood and adolescence and High School Dropout: University of California, Davis2010.

3. De Witte K, Cabus S, Thyssen G, Groot W, van den Brink HM. A critical review of the literature on school dropout. Educational Research Review. 2013;10(0):13-28.

4. Freudenberg N, Ruglis J. Reframing school dropout as a public health issue. Prev Chronic Dis. 2007 Oct;4(4):1-11.

5. Mäki N, Martikainen P, Eikemo T, Menvielle G, Lundberg O, Östergren O, et al. Educational differences in disability-free life expectancy: a comparative study of long-standing activity limitation in eight European countries. Soc Sci Med. 2013;94:1-8.

6. Mackenbach JP. Health inequalities: Europe in profile. London: COI.2006.

7. Bradshaw CP, O'Brennan LM, McNeely CA. Core competencies and the prevention of school failure and early school leaving. New Directions for Child and Adolescent Development. 2008;122:19-32.

8. Ensminger $M$, Slusarcick $A$. Paths to high school graduation or dropout: A longitudinal study of a first grade cohort. Sociol Educ. 1992;65:95-113.

9. Jimerson S, Egeland B, Scroufe L, Carlson B. A prospective longitudinal study of high school dropouts; Examining multiple predictors across development. J School Psychol 2000;38:525-49.

10. Gilmore M, Hawkins J, Day L, Catalano R. Friendship and deviance: New evidence on an old controversy. J Early Adolesc 1992;12:80-95.

11. Patterson GR, DeBaryshe BD, Ramsey E. A developmental perspective on antisocial behavior. American Psychologist. 1989;44(2):329-35.

12. Sewell J. School refusal. Fam Physician. 2008;37:406-8.

13. Bronfenbrenner $U$, Morris P. The ecology of developmental processes. In: Damon, editor. Handbook of child psychology. Hoboken, NJ: Wiley; 1998. p. 993-1028.

14. Rutter M. Pathways from childhood to adult life. J Child psychology psychiatry. 1989;30:23-51.

15. Atkinson L, Beitchman J, Gonzalez A, Young A, Wilson B, Escobar M, et al. Cumulative Risk, Cumulative Outcome: A 20-Year Longitudinal Study. PLoS One. 2015;10(6):e0127650.

16. Townsend L, Flisher AJ, King G. A systematic review of the relationship between high school dropout and substance use. Clinical Child and Family Psychology Review. 2007;10(4):295-372.

17. Theunissen M-J, Bosma H, Verdonk P, Feron F. Why Wait? Early Determinants of School Dropout in Preventive Pediatric Primary Care. PLoS One. 2015;10(11):e0142315.

18. Theunissen M-J, Griensven van I, Verdonk P, Feron F, Bosma H. The early identification of risk factors on the pathway to school dropout in the SIODO study: a sequential mixed-methods study. BMC Public Health. 2012;12(1):1033.

19. European Council. Council Conclusions of 5 May 2003 on Reference Levels of European Average Performance in Education and Training (Benchmarks). Official Journal of the European Union. 2003;46(C 134/03):3-4.

20. United Nations Educational Scientific and Cultural Organization (UNESCO). The revised International Standard Classification of Education (ISCED 2011). Paris 5 September 2011.

21. Theunissen M-J, de Man I, Verdonk P, Bosma H, Feron F. Are Barbie and Ken too cool for school? A casecontrol study on the relation between gender and dropout. The European Journal of Public Health. 2015;25(1):57-62.

22. Bosma H, Theunissen M-J, Verdonk P, Feron F. Low control beliefs in relation to school dropout and poor health: findings from the SIODO case-control study. BMC Public Health. 2014;14(1):1237.

23. McCall L. The complexity of intersectionality. Signs. 2005;30(3)(3):1771-800.

24. Green J, Thorogood N. Qualitative Methods for Health Research. 2 ed. Los Angeles: Sage Publications; 2009. 
25. Guest G, Bunce A, Johnson L. How many interviews are enough? An experiment with data saturation and variability. Field methods. 2006;18(1):59-82.

26. Strauss A, Corbin J. Basics of qualitative research: Grounded theory procedures and techniques: Newbury Park, California: SAGE Publications; 1990.

27. Braun V, Clarke V. Using thematic analysis in psychology. Qualitative Research in Psychology. 2006;3(2):77-101.

28. Shochet IMSTR. The Impact of Parental Attachment on Adolescent Perception of the School Environment and School Connectedness. Australian \& New Zealand Journal of Family Therapy. 2007;28(2):109-18.

29. Blondal KSAS. Parenting practices and school dropout: a longitudanl study. Adolescence. 2009 Winter2009;44(176):729-49.

30. Kitzmann KM, Gaylord NK, Holt AR, Kenny ED. Child witnesses to domestic violence: A meta-analytic review. Journal of Consulting and Clinical Psychology. 2003;71(2):339-52.

31. Winstok Z. Effects of Childhood Experience of Violence Between Parents and/or Parent-to-Child Violence on Young Israeli Adults' Global Self-Esteem. Violence and Victims. 2015;30(4):699-713.

32. Turner HA, Shattuck A, Finkelhor D, Hamby S. Effects of Poly-Victimization on Adolescent Social Support, Self-Concept, and Psychological Distress. Journal of Interpersonal Violence. 2015 June 1, 2015.

33. Bandura A. Self-efficacy: Toward a unifying theory of behavioral change. Psychological Review. 1977;84(2):191-215.

34. Griffith M, Dubow E, Ippolito M. Developmental and Cross-Situational Differences in Adolescents' Coping Strategies. Journal of Youth and Adolescence. 2000 2000/04/01;29(2):183-204.

35. Hermes J, Naber P, Dieleman A. Leefwerelden van jongeren. Coutinho, Bussum. 2007.

36. Baumeister R, Leary MR. The need to belong: Desire for interpersonal attachments as a fundamental human motivation. Psychological Bulletin. 1995;117:497-529.

37. Allen JP, Porter MR, McFarland CF, Marsh PA, McElhaney KB. The two faces of adolescents' success with peers: Adolescent popularity, social adaptation, and deviant behavior. Child Development. 2005;76:747-60.

38. Hussong AM, Hicks RE, Levy SA, Curran PJ. Specifying the relations between affect and heavy alcohol use among young adults. Journal of Abnormal Psychology. 2001;110(3):449-61.

39. Brown MR, Higgins K, Paulsen K. Adolescent Alienation: What Is It and What Can Educators Do About It? Intervention in School and Clinic. 2003 September 1, 2003;39(1):3-9.

40. Lee VE, Burkam DT. Dropping out of high school: The role of school organization and structure. American Educational Research Journal. 2003;40(2):353-93.

41. Lee VE, Smerdon BA, Alfeld-Liro C, Brown SL. Inside large and small high schools: Curriculum and social relations. Educational Evaluation and Policy Analysis. 2000;22(2):147-71.

42. Skinner EA, Pitzer JR. Developmental dynamics of student engagement, coping, and everyday resilience. Handbook of research on student engagement: Springer; 2012. p. 21-44.

43. Smyth J, Hattam R. Early School Leaving and the Cultural Geography of High Schools [1]. British Educational Research Journal. 2002 2002/06/01;28(3):375-97.

44. Sameroff A. A Unified Theory of Development: A Dialectic Integration of Nature and Nurture. Child Development. 2010;81(1):6-22.

45. Wang JZ. Female Gang Affiliation: Knowledge and Perceptions of At-Risk Girls. Int J Offender Ther Comp Criminol. 2000;44(5):618-32.

46. Esbensen F-A, Deschenes EP, Winfree LT. Differences between Gang Girls and Gang Boys: Results from a Multisite Survey. Youth Soc. 1999;31(1):27-53.

47. Hay I. Gender Self-concept Profiles of Adolescents Suspended from High School. Journal of Child Psychology and Psychiatry. 2000;41(3):345-52.

48. Ozyurt S. Negotiating Multiple Identities, Constructing Western-Muslim Selves in the Netherlands and the United States. Political Psychology. 2013;34(2):239-63.

49. Hannah J-AS, Kahn SE. The relationship of socioeconomic status and gender to the occupational choices of grade 12 students. Journal of Vocational Behavior. 1989;34(2):161-78.

50. Bosma H. Socio-economic differences in health: are control beliefs fundamental mediators. Social inequalities in health, new evidence and policy implications. 2006:153-66. 



\section{Chapter 7}

\section{"Don't you have faith in them?" \\ A focus group study with stakeholders in the process toward school dropout}

Theunissen M, van Maren M, Bosma H, Feron F, Verdonk P. "Don't you have faith in them?" A focus group study with stakeholders in the process toward school dropout (submitted for publication). 


\section{Abstract}

\section{Background}

School dropout is still understudied from a public health perspective. To contribute to the future development of public health interventions to prevent school dropout as early as possible, we explored how parents, educational and health care stakeholders view the reasons for dropping out of school and theirs and each other's roles in preventing school dropout at an early age.

\section{Methods}

We conducted five homogeneous focus groups with parents, stakeholders from education and health care. The interview guide was based on health-related determinants associated with school dropout, found in previous studies. A content analysis was performed.

\section{Results}

We identified four themes; (1) growing into responsibilities, (2) meeting the norm, (3) building a bridge and (4) bending the rules. According to stakeholders, children should learn how to take responsibility for their own learning process, age-appropriate. Expecting too much, may lead to fear of failure and decrease self-confidence. For belonging to a peer group and fitting in the school system, which provides self-confidence and sufficient support, youngsters must meet the established norm. Unclear professional roles, medical confidentiality and mutual mistrust may hinder stakeholders to cooperate in preventing school dropout. Both the educational and health care system are rigid, performance-driven systems, bound to limiting rules, which had to be bended to provide tailored support.

\section{Conclusions}

To prevent school dropout, the individual child and his or her environment should be involved. Mutual trust between stakeholders must be restored to cooperate for the sake of the child. The youth health care physician may be a linking pin. Educational and health care systems need to be facilitated sufficiently and be more flexible so that professionals can provide tailored support, taking into account individual differences. 


\section{Background}

Early school leaving is a complex problem that affects individual youngsters, their families and society. For individuals, dropping out of school is associated with many physical and mental health problems, reduces their prospects on the labour market and contributes to socioeconomic health differences (1-3). It is therefore not only a problem of education, but also of public health. Yet, in the Netherlands the topic of school dropout is heavily understudied from a public health perspective. The Dutch government has implemented several policies to reduce early school leaving but most interventions are targeted toward monitoring and enforcing school attendance, and occur late in the dropout process (4). This study explores the stakeholders' views about possibilities of preventive interventions early in life targeting toward children's and their families resilience and strengths.

In our previous studies, including an interview study with 18 to 23 year old youngsters (dropouts and delayed graduates), we gained an in-depth understanding into the pathways to school dropout from the perspective of the youngsters (5-7). However, parents and educational professionals may have different views to the causes and solutions than the youngsters themselves. For instance, students may see boredom as a central cause for their decreased motivation and dropping out of school, whereas teachers may believe that students are making excuses for their failure to graduate (8, 9). Moreover, little is known about the perspectives of health care (HC) professionals.

Since parents and stakeholders from education and $\mathrm{HC}$ are all involved in a child's development, their perspectives may complement our understanding of the school dropout process. Furthermore, it will provide information about education and HC policies and the way they are carried out by professionals. Bronfenbrenner stated that there are different levels of environmental influences that can directly or indirectly affect a child's development (10). Family, friends and teachers directly interact with the child but also with each other. Therefore, a child's development can also be influenced by the way parents and teachers work together for the sake of the child. Parents and teachers may experience difficulties in cooperating, especially when children are in need for extra support or show behavioural problems at school $(11,12)$. Furthermore, the child's development can indirectly, through the action of others, be influenced, i.e. via a school systems organizational practices, which can facilitate or frustrate peer relationships (13) or governmental regulations which only finance the educational and health care organisations' core activities, leaving insufficient time for cooperation between them (14).

Because the topic of school dropout is relatively new as public health issue, we conducted semi-structured focus group interviews with parents, stakeholders from education and stakeholders from HC, respectively (15). The following research questions are addressed: "How do parents, stakeholders from education and stakeholders from health care view the reasons for dropping out of school and their own and each other's 
roles in preventing school dropout at an early age?" Results will contribute to the future development of public health interventions to prevent school dropout as early as possible, preferably before further harm occurs.

\section{Methods}

\section{Study design}

This subsequent focus group study is based on a quantitative case-control study, and individual interviews with young adults ( $18-23$ years old) who dropped out of school or graduated delayed (16). (Figure1).

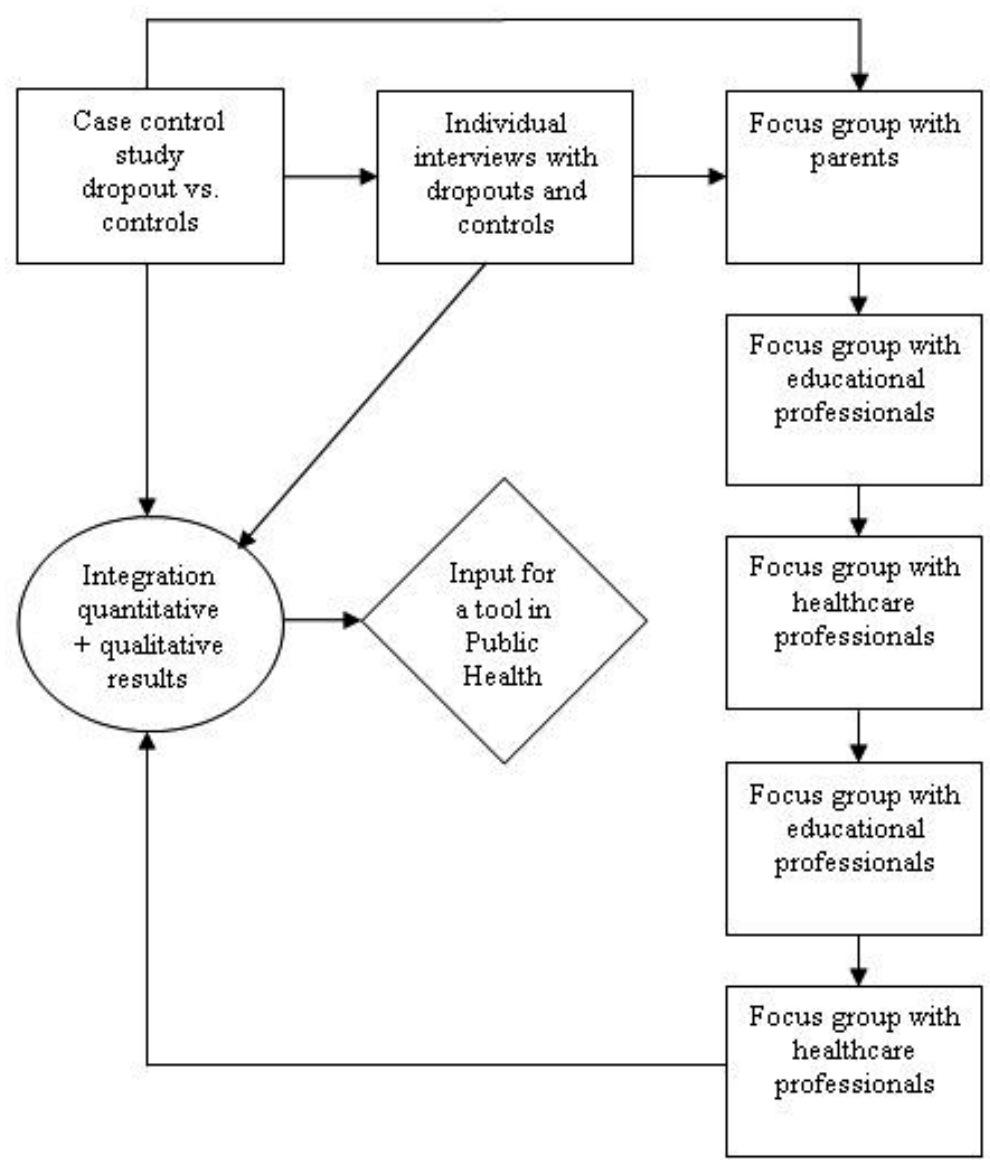

Figure 1 Flowchart of the "Stay in or drop out" (SIODO) study.

In each focus group, information from the previous focus groups was presented to validate the findings with other stakeholders. Each focus group consisted of other participants. 


\section{Participants}

Focus groups were conducted with parents, educational professionals and HC professionals. In each focus group, other participants took part. Table 1 shows the participants' characteristics of the different focus groups.

Table 1 Characteristics of the Focus groups' participants.

\begin{tabular}{|c|c|c|c|c|c|}
\hline Stakeholders & $\begin{array}{l}\text { FG1 } \\
\text { Parents }\end{array}$ & $\begin{array}{l}\text { FG2 } \\
\text { Education }^{1}\end{array}$ & $\begin{array}{l}\text { FG3 } \\
\text { Health care }\end{array}$ & $\begin{array}{l}\text { FG4 } \\
\text { Education }^{1}\end{array}$ & $\begin{array}{l}\text { FG5 } \\
\text { Health care }\end{array}$ \\
\hline \multicolumn{6}{|l|}{$\operatorname{Sex}(n)$} \\
\hline Male & 2 & 2 & 0 & 4 & 1 \\
\hline Female & 5 & 3 & 7 & 5 & 8 \\
\hline \multicolumn{6}{|l|}{ Age group (years) (n) } \\
\hline$<40$ & 0 & 0 & 1 & 1 & 2 \\
\hline $40-50$ & 4 & 0 & 0 & 2 & 1 \\
\hline$>50$ & 3 & 5 & 6 & 6 & 6 \\
\hline Work experience (years) (n) & n.a. & & & & \\
\hline$<10$ & & 0 & 1 & 2 & 1 \\
\hline 10 to 20 & & 1 & 1 & 3 & 1 \\
\hline $20-30$ & & 0 & 3 & 0 & 3 \\
\hline$>30$ & & 4 & 2 & 4 & 4 \\
\hline
\end{tabular}

FG = focus group. ${ }^{1}$ Teachers of primary- secondary- and intermediate vocational education, student counsellors, a janitor, school truancy officers and dropout guidance counsellors. ${ }^{2}$ Youth health care physicians and nurses, a general practitioner, a paediatrician, social workers, youth workers, youth psychologists and an educationalist.

Selection of parents was done through purposive sampling and personal contacts (17). We approached 141 parents from youngsters who dropped out of school, by e-mail (15) and mail (126). Addresses were provided by the compulsory education department of the municipality of Eindhoven, a city in the South-East of the Netherlands. Since this yielded only 3 participants, of whom one cancelled two days before the focus group, we reached out through social media (Facebook and Linked in) and the personal network. Despite this convenience sampling, the variation was rich regarding sex, ethnic background and educational level of parents and their children. Finally, 7 parents participated, of whom two were parent couples who were approached personally. One parent had a non-Western background. All participants had one or more children who graduated with a delay or dropped out of school, but re-entered school later on. Participants did not meet before. The participants received a $€ 15$, - cinema voucher as an incentive and we refunded their travel expenses. As a consequence of recruitment problems, we conducted one instead of two focus groups with parents. 
The stakeholders from education and $\mathrm{HC}$ were recruited through the professional network of the first author, who also works as a youth health care (YHC) physician (see Box 1), and through snowball sampling. We used purposive sampling to include participants from a diverse range of backgrounds, views and experiences and to create a group with a wide variety of professions and affinity with different child ages (17). Participants were contacted by e-mail or phone and did not receive an incentive. Some participants knew each other already from their professional network which may have maximized the interaction between the participants (18).

Box 1 the Dutch youth health care system

The preventive Dutch youth health care (YHC) physicians and nurses offer routine health examinations and
anticipatory guidance, free of charge, to all children between birth and 18 years (19). They are closely
cooperating with primary and secondary schools and with curative health care professionals.

\section{Focus groups}

From March to June 2015 we conducted five homogenous focus groups with duration of 2 hours at a Public Health Service in the South East of the Netherlands. Homogenous focus groups provide more power balance than heterogeneous groups, which allows participants to speak more freely and maximizes the interaction between them (18). All parents had children with school difficulties and the professionals were similar in working with children from an educational or $\mathrm{HC}$ perspective. To establish an informal atmosphere, to talk more easily, we provided drinks, sweets and cake (a light meal during the first $\mathrm{HC}$ focus group). We explored the range of views about dropping out of school and which guidance to prevent school dropout may be useful and feasible with parents, and stakeholders from education, and $\mathrm{HC}$, respectively (Figure 1). We particularly chose this order because it reflected the order of problem solving dimensions in daily practice: parents first, then teachers, and finally health care professionals. Prior to each focus group, we showed determinants of school dropout during the life course from previous studies (5-7). Furthermore, in each focus group, information from the previous focus groups was presented to validate the findings with other stakeholders (data triangulation) (20). This enabled us to further interpret and understand our earlier focus group findings. In all focus groups, earlier findings and first conclusions from previous focus groups were recognized by the participants and considered relevant and important. The groups consisted of five to nine participants, which is large enough to explore a wide range of views held by parents and stakeholders from education and $\mathrm{HC}$ and small enough to maintain control and encourage interaction (15). 
Box 2 the Dutch educational system

\section{Procedure}

The focus groups were Dutch spoken, moderated by MT (researcher and YHC physician), and observed by MvM (physician) who also took notes. The last focus group with stakeholders from $\mathrm{HC}$, in which we particularly zoomed in on the role of the different stakeholders in preventing school dropout, was moderated by PV (psychologist) and observed by MT and MvM. We used an interview protocol with a topic list and fictitious cases based on the health-related determinants associated with school dropout, found in previous studies (5-7) (Table 2). 


\section{CHAPTER 7}

Table 2 Interview guide

\begin{tabular}{ll}
\hline Opening and introduction & $\begin{array}{l}\text { Information about the expectations of the focus group, confidentiality, informed } \\
\text { consent, approval for recording, duration, demographics. } \\
\text { Presenting the previous results of the SIODO study }\end{array}$ \\
Opening questions & "What do you think of the findings?" \\
"What anecdotes or examples from your work or daily life come to your mind?" \\
Topics & The importance of a basic qualification \\
Education & The educational system, responsibilities \\
Health care & The health care system, access to care, responsibilities \\
Cooperation & The cooperation between parents, school and health care \\
Responsibilities & Who is responsible for what? \\
Hard to reach families & How can we motivate children, who are hard to reach? \\
Intervention & What kind of support is needed and at what time (age)? \\
Closure questions & "Are there any further comments regarding this topic?" \\
Evaluation & Brief evaluation of the focus group
\end{tabular}

${ }^{1}$ Focus groups 1 to 3 were shown the previous results of the SIODO study. Focus groups 4 and 5 were also shown the themes that emerged from the previous 3 focus groups.

\section{Ethics statement}

Approval for conducting this study was granted by the Medical Ethics Committee of Maastricht University (METC 11-4-099, decision 22 August 2011).

\section{Data analysis}

All focus groups were tape-recorded after consent, transcribed verbatim and inserted in Excel to systematically analyse the data. During data collection on site notes were taken (18). Researcher triangulation was applied for coding, clustering and analytic decisions (21). All authors read the notes and transcripts to familiarise themselves with the data and to collect and discuss ideas. MT and MvM hand-coded the first three transcripts separately, using open coding and designed the code tree (Table 3). They compared their codes and in case of a disagreement, negotiation and clarification was applied until consensus was reached. Generally, there was a high level of agreement. The code tree was discussed with all authors. Two transcripts were hand-coded by MT and discussed with PV. The first three transcripts were analysed thematically in accordance with the grounded theory approach (22) to identify patterns and categories in the data $(17,20)$. The codes were combined into overarching categories or subthemes (axial coding). Subsequently, a profound inductive summary of each subtheme could be developed 
and four major themes emerged, under which all codes could be placed. Between each focus group MT, MvM and PV discussed the findings to present them in the next focus group. (18). After analysing the subthemes for each focus group, the themes were compared between groups and analysed for similarities and discrepancies. These findings were discussed with all authors for new views and ideas.

Table 3 Code tree

\begin{tabular}{|c|c|c|}
\hline Codes & Subthemes & Themes \\
\hline Competences & Child factors & \\
\hline \multicolumn{3}{|l|}{ Responsibility } \\
\hline \multicolumn{3}{|l|}{ Self-confidence } \\
\hline Behaviour & & Growing into responsibilities \\
\hline \multicolumn{3}{|l|}{ Health } \\
\hline Parental role model & (Social) environment & \\
\hline \multicolumn{3}{|l|}{ Drawing boundaries } \\
\hline \multicolumn{3}{|l|}{ Expectations toward the child } \\
\hline \multicolumn{3}{|l|}{ Stressful life events } \\
\hline Being understood & Belongingness & \\
\hline \multicolumn{3}{|l|}{ In-and exclusion } \\
\hline \multicolumn{3}{|l|}{ Customized care } \\
\hline Relationships & & Meeting the norm \\
\hline \multicolumn{3}{|l|}{ Diversity } \\
\hline Motivation/stimulation & Support & \\
\hline Parents & Cooperation & \\
\hline \multicolumn{3}{|l|}{ Education } \\
\hline \multicolumn{3}{|l|}{ Health care } \\
\hline Trust & & Building a bridge \\
\hline Access to care & Task conception & \\
\hline \multicolumn{3}{|l|}{ Continuity in care } \\
\hline \multicolumn{3}{|l|}{ Norms and values } \\
\hline \multicolumn{3}{|l|}{ Responsibility } \\
\hline \multicolumn{3}{|c|}{ Expectations toward other stakeholders } \\
\hline Basic qualification & Society & \\
\hline \multicolumn{3}{|l|}{ Job opportunities } \\
\hline \multicolumn{3}{|l|}{ Health care system } \\
\hline Educational system & & Bending the rules \\
\hline \multicolumn{3}{|l|}{ Society } \\
\hline Conditions & Rules and regulations & \\
\hline \multicolumn{3}{|l|}{ Limitations } \\
\hline Privacy and confidentiality & & \\
\hline
\end{tabular}




\section{Findings}

Four major themes emerged about how parents, stakeholders from education and stakeholders from $\mathrm{HC}$ view the reasons for dropping out of school and how they view their own and each other's roles in preventing school dropout at an early age: (1) growing into responsibilities, (2) meeting the norm, (3) building a bridge and (4) bending the rules.

\section{Growing into responsibilities.}

The amount of responsibility a child can handle depends on the child's age and abilities. According to educational and $\mathrm{HC}$ professionals, children learn to deal with responsibilities at a young age and nurturing them plays a large role in this. Parents mentioned that being a role model for younger siblings or taking care of them makes children grow socially and emotionally and increases self-confidence. However, when children cannot handle those responsibilities, they may be overwhelmed.

"I think, when a little brother or sister is born, children want to become a rolemodel. They want to be the big brother or sister and want to be admired; they want to be looked up to". "They are going to do their very best" (Parents, FG 1)

"Well, you do saddle such a little child with a big responsibility. Yes, I believe it might result in a fear of failure, when there is too much projected on the child, such as keep telling you are the oldest one, so you must be responsible. That can have a negative impact". (Parent, FG 1)

Children imitate their parents' behaviour, who mostly are their role models in early childhood. Therefore, if parents are disrespectful toward teachers, so are their children and if parents easily keep their children at home, i.e. for extra holidays, children will gain less responsibility toward school attendance. Educational professionals questioned parents' parenting skills, referring to a lack of consistency and authority over the child. Parents are not in charge anymore and set no limits to their children's behaviour. This lack of rules at home may lead to problems with accepting authority at school.

"Well, it starts in the stroller. I see parents with their child eating a fat snack in the buggy. I think it starts there. If you cannot control your baby or toddler, you cannot control your adolescent. And it actually starts with setting limits and not being a friend but a parent". (Educational professional, FG 2)

Some educational stakeholders believed that learning responsibilities should be given to the children from primary school onwards; so they will be ready for planning and organising their homework in secondary school. Others felt that children must play in primary school. Giving a child too much responsibility or having too high expectations, especially regarding educational achievement may put children under pressure, decreases their 
self-esteem and may lead to a fear of failure and loss of school motivation. According to educational and $\mathrm{HC}$ professionals, some parents gave their children extra lessons to attain a higher score at the final aptitude test on primary school. They even put pressure on teachers to give their children a higher rate so that they could enter a higher school level in secondary education (Box 2).

"And if you notice how parents respond to the aptitude test on primary school; they send their children to tutoring just for a higher score. I think, why? Don't you have faith in them? Just let that child do that test and it will be fine. But no, parents come in a spasm too. Children must go to the senior general secondary school, that they emotionally will suffer does not matter anymore". (Health care professional FG 3)

$\mathrm{HC}$ professionals mentioned that children with learning or behavioural problems are sometimes overestimated by teachers, who keep them on a regular school too long.

"I do see them struggle with children with learning or behavioural problems but sometimes they really keep those kids in a regular school for too long. Actually, I think that destroys a lot. Those children lose motivation for school and are lying in bed all day. And this already applies to children of 9, 10 years old" (Health care professional, FG 3)

However, having low expectations and taking away children's responsibilities, will show less faith in the child's 'capabilities and may lead to frustration. According to educational professionals, some colleagues underestimate the intelligence of migrant children because of a language deficit. Parents mentioned paying more attention to their sick and disordered children, whom they considered particularly vulnerable, than to their healthy children, although they are aware that overprotection may make children feel insecure. According to an educational professional, all adolescents want to show their trustworthiness, including those with a developmental disorder, such as autism spectrum disorder:

"Adolescents with autism also want to show that they can handle responsibilities. But those parents are very protective. Well, that may lead to conflicts it. I think confidence is important, with confidence, between parents and children, and children and school, everything will be relaxed. Then I think okay, you like to go to school, you like to join the lessons, you show what you can, show a little bit more. Sometimes they will cross the line. But by setting limits and still giving responsibility, and confidence, they can grow". (Educational professional, FG 4) 


\section{Meeting the norm}

Belongingness is a strong motivation for finishing school, but youngsters have to conform to the norm to be included in peer groups and to match with the school system. All stakeholders and parents agreed that most children, especially in puberty, want to belong to a peer group. Educational professionals mentioned that adolescents start forming groups in secondary school. Moreover, a YHC nurse added that the pressure to be included in such a group is high. Adolescents who do not conform to the group's norm risk being excluded. This applies to looks, but also to sexual orientation, learning problems, diseases and developmental disorders, such as autism spectrum disorder.

"My son has a type of autism. I do not know eh about the rest of the group, but my son knows damn well what it is not to belong, because they do not understand him. And there is really nothing you can do about that. He is now 23 years old and if something goes wrong, he immediately says that he does not fit in"(Parent, FG1)

Schools apply standards and norms regarding learning results and behaviour, and not conforming to them may lead to exclusion. According to HC professionals some preschool children, exhibiting deviant behaviour in a playgroup, were refused in kindergarten and a Moroccan boy with a conduct disorder, faced difficulties to be admitted at a new school. Educational professionals explained that little rebellious boys are considered cute by adults, which encourages their externalizing behaviour but then later, deviant behaviour has become a pattern. In school however, they are not allowed to exhibit tough behaviour and since most teachers are female, they expect children to be calm and quiet. HC professionals think that girls are more suitable for the educational system because they are conscientious, and are more intrinsic motivated for school than boys. Male teachers may make a difference, because they better identify themselves with hyperactive boys:

"A male primary school teacher once told me that he would get three five-yearold boys with ADHD in his class after summer vacation. The first three weeks he was looking around and thought, I don't know where those kids are, but they sure aren't in my class. But a male teacher plays football in the school-yard and let them play rough-and-tumble, that is a completely different way of handle those kids. Female teachers always let them sit down quietly and tinker something" (Health care professional, FG5)

In all focus groups, the opinion predominated, that education works sufficiently for 'average children', but children who perform better or worse do not fit in the traditional system. 
"I think that $80 \%$ is the average student and $20 \%$ are the outliers. I think that these $20 \%$ get into trouble. There is not enough guidance and knowledge for them in primary school". "And not enough budget, not enough teachers of course." (Parents, FG1)

A HC professional mentioned that the educational content needs to be adapted to the child and not the other way around as it mostly is today. An educational professional believed that learning problems do not exist, as long as children's talents are developed. School must be a stimulating environment, with respect to differences so children will be intrinsically motivated. Other educational professionals also think that children need to be challenged at their own level, so they do not get bored. Stakeholders from HC and education mentioned that it is also important to align oneself with the child's social environment and its norms and values, because it may explain underperformance such as in the quote below.

"Sometimes you have to detach a student from home because loyalty plays a role. I once experienced, that a boy did not want to pass his exam. First we thought he was afraid to fail, you know. Because he always joined classes, always participated and still got bad results. Then it turned out that he did not really want to pass his exam. Because if he did, he would be the only one in the family with a diploma. So, then you also know how they talk about people with a diplo$m a^{\prime \prime}$. (Educational professional, FG2)

$\mathrm{HC}$ professionals discussed that to provide personalized care, it is important to connect to parents and youngsters. In case of migrant families, this also means being culturally sensitive, providing information from the perspective of the migrant group and using a translator, if necessary. Especially migrant families, who are used to solve problems within their family, find it difficult to accept professional support. Dutch families may also face difficulties in accepting psychological support, because of its stigma. Generally, more girls and feminine boys with a depression or an anxiety disorder end up at the psychologist. Tough boys drop out of care easily, especially when they need to talk about the origin of their problems. A therapy focused on solutions may fit them better.

"The feminine men, eh, feminine boys go to the psychologist of course, boys with anxiety and depressive symptoms. Boys with externalizing problems are not so easily sent to a psychologist. [.....] I would do a completely different therapy here. It is not so important to search for the cause; I would focus on the solution instead. [.....] I think if you focus on the solution, when a boy has externalizing behavioural problems, things will be done much faster than when you focus on how it occurred. That is what I often think with this kind of problems, because they will drop out, and they do not feel heard or understood by care professionals". (Health care professional, FG5) 


\section{Building a bridge}

Building a bridge is about task conception and how this influences cooperating. Professional roles are not always clear and may confront professionals with a dilemma, i.e. regarding the teachers' role in case of a problematic family situation. Some educational professionals believed that unless there is a reason in terms of bad school results, a teacher has no right to interfere with families. However, most teachers considered signalling educational, social and emotional problems to be their job and felt obliged to interfere if necessary. According to $\mathrm{HC}$ professionals, teachers find it hard to discuss their suspicions about family problems or learning disorders with parents, especially when they expect to disagree with the children's needs. Some teachers mentioned to take preventive measures such as entering into the dialogue along with a colleague, when they feared aggression, others postponed having a conversation with parents and kept collecting information to confirm their suspicions. However, HC professionals felt, in case of worries, teachers to be responsible for involving parents from the beginning:

"They have already discussed a lot and then they have a conversation with the parents, who very often become overwhelmed by what is being told and what is being discussed, because they have not been involved in that process until now. Because it's been so difficult, because many teachers find it difficult, and they are the first to contact parents to start a dialogue, and doing this in the right way. Without blaming, without accusations, without putting emotions into it". (Health care professional, FG5)

Regarding children with problem behaviour, the boundaries between the educational and public health domains may be indistinct. HC professionals sometimes felt that teachers went beyond their professional boundaries, i.e. referring a child with externalizing behaviour for medication to the general physician or trying to figure things out themselves instead of consulting HC professionals. Teachers on the other hand, questioned the sense of responsibility of $\mathrm{HC}$ professionals, who failed to ask them for information about children. Furthermore, teachers mentioned that YHC professionals may have different understandings of their role; some consider the whole system, but others only focus on the individual child:

"I join different consultation teams and mostly there is a YHC physician present. And then you notice that there are many differences in the way the YHC physician work [...] Some physicians say, I studied that family and I notice that this is the third child in a row that starts with the same problems and a younger child already starts playing truant in primary school. Then I think you see this family in a different way than your colleague, who only looks at the individual child and says she really suffers from her period, so it is possible that she is absent due to illness. 
You know, it depends on how a YHC physician sees her profession, what she counts to be her responsibility". (Educational professional, FG2)

Educational professionals experienced the cooperation with HC professionals differently. From being situated in the same building and having short communication lines to not knowing whether there is medical, social or psychological aid involved. Parents mentioned not knowing what the YHC had to offer and how it could be reached. However, they envision that YHC professionals take a mediating role between parents and school and monitor whether school follows the given advice. All stakeholders including parents felt that YHC professionals should be a linking pin, organize a multidisciplinary meeting and connect other $\mathrm{HC}$ professionals, parents, and teachers to each other.

"If the YHC physician knows what is going on in a family, with a child, she can contact school, cooperate with school and allow school to do something about it. Or, allowing, demanding that school does something with it" (Parents, FG1)

Parents mentioned that they want to be involved in case teachers discuss their children's problems with colleagues or HC professionals. However, according to HC professionals, teachers often fail to involve parents when signaling behavior or learning problems. Some tension in the parent-teacher relationship exists, due to mistrust in each other's qualities. Educational professionals believed that parents often did not recognize what support their child with learning problems needs. They think that parents seem to distrust school's good intentions, keep off the necessary assistance or pressure teachers into a solution. Parents on the other hand, did not feel taken seriously by teachers, because teachers did not listen to them, downplayed their problems or neglected professional advice. They think that teachers' arrogance is an obstacle in cooperating with school.

"What I really think is important, is that primary school teachers should not be so authoritarian and start listening to parents". (Parent, FG1)

"We experienced the same problem at primary school, there was an advice from a psychologist, but the teacher did not listen. She did exactly the opposite and it didn't turn out well". (Parent, FG1)

Educational and $\mathrm{HC}$ professionals agreed that the transition from primary school to secondary school is often difficult for children. They change a safe environment for a mostly larger school with different (social) dynamics. A good transfer including information about a student's need for support may be necessary. According to educational professionals, important information about students' needs and mental health is often withheld. Parents want their child to make a fresh start at the new school, without any stigma.

"We have experienced a few times, that uh schools wanted to give a student a fresh start and did not provide information about special needs. Oh, oh. That is ouch. Yes they do not have to discuss everything in detail, but they have to dis- 
cuss it. Knowing absolutely nothing about a student's need for support went completely wrong a couple of times. (Educational professional, FG4)

\section{Bending the rules}

Both the educational and $\mathrm{HC}$ system are bound to rules and regulations, which can provide guidance in case of support for the average children, but can also be limiting when it comes to provide tailored support. In the educational and the HC focus groups, stakeholders mentioned that they provided sufficient care when they stepped out of their comfort zone and bended the rules:

"You see, when someone doesn't show up for a consultation, my boss does not allow me to contact them anymore after two times no show. But I don't listen to him, and I'll do it anyway." (Health care professional, FG3)

"When I am worried about a child, I want to make a change, but some laws are opposing. There has to be a minimal amount of truancy before I am allowed to interfere, but why can I not tackle the problem when a child plays truant for only half an hour if I am worried about him" (Educational professional, FG2)

According to the educational professionals the educational system is rigid, which hinders innovation. For example, a group of French teachers had prepared a continuous learning pathway to decrease the transitional gap to vocational education, but existing teaching materials did not correspond to it and could not be changed. Furthermore, despite educating a new generation, schools are always the last to implement technical innovations. Some educational stakeholders mentioned that teachers are rather conformist and obedient and should stand up for better circumstances, but others mentioned that with standing up against the rigid educational system they may risk their job:

"Aren't we too obedient with filling in all those action plans? I also think: the hell with it. Seriously, when I consider how many meetings there are, and all directors and all internal counsellors, everyone must be present. And what are the outcomes? Zero point zero. Just give me that money, and I will spend it on my kids at my school, then I'm happy. [.....] Who is standing up and says: I am breaking with all these rules. I am going to do what is best for this child. [.....] Professionals in education stand up and take your responsibility". (Educational professional, FG2)

The rules for educational financing are bureaucratic and based on performance, which makes including youngsters with learning or behaviour problems difficult. An educational professional explained that before a school receives money to support a youngster who needs additional support, huge files with the student's developmental perspectives, tests and the need for support are required for assessment by a regional cooperative association. Furthermore, the students' current school has to ascertain that 
the child can attend the right school when they cannot support the student themselves. However, students with behavioural problems are hard to transfer because they are at higher risk for dropping out of school, and schools are reluctant to take these students in as they will receive less money when a student drops out of school.

"We present our difficult students to intermediate vocational education and tell them that they have to take them. We know that they are very problematic students and hope it will turn out right. But when things go wrong, the school of intermediate vocational education will be punished. This culture of judgement, that has to disappear. If you take problematic students, you have to be rewarded. Moreover, the whole educational chain from 4 years old to 23 years old is responsible for the development of a child". (Educational professional, FG2)

The HC professionals felt bound by rules, confidentiality being one of them. Only when a child is in danger, and hence, a conflict of duties occurs, professionals are allowed to deviate from professional secrecy. Parents mentioned privacy to be very important and necessary for a trustful patient-doctor relationship. The educational professionals however, experienced medical confidentiality to be an obstacle for good cooperation between school and $\mathrm{HC}$ and blamed $\mathrm{HC}$ professionals to hide behind privacy rules.

"It regularly occurs that medical confidentiality interferes with the transparency of the problems. Especially youth care misses the synchronisation with education. I often think it is a missed opportunity". (Educational professional, FG4)

In the Netherlands, youngsters must obtain a basic qualification (defined as a diploma), which is considered to be the minimum educational level for a job on the labour market. Parents feel this to be unfair, for it would be better to judge people by their qualities and not all competences manifest in diplomas. Educational professionals subscribed the importance of a basic qualification, but to motivate children and give them prospect of a positive future, they would prefer if the labour market provided more low-skilled job opportunities.

"Perhaps you must not say, we want a basic qualification, but there have to be jobs for lower educated students". (Educational professional, FG2)

\section{Discussion}

In answering the questions how parents, stakeholders from education and stakeholders from (youth) health care view the reasons for dropping out of school and perceive their own and each other's roles in preventing school dropout at an early age, we found four major themes (1) growing into responsibilities, (2) meeting the norm, (3) building a bridge and (4) bending the rules. For a successful school career, all stakeholders including parents felt that children must learn how to take responsibility for their own learn- 
ing process, appropriate for their age. Expectations about youngsters' learning achievements should not be set too high or too low, otherwise this may lead to fear of failure and decrease self-confidence. Youngsters must meet the established norm to belong to a peer group and to fit into the school system, which is important for youngster's motivation and for receiving sufficient support. The professional roles in preventing school dropout are not always clear, neither are the boundaries of the educational and $\mathrm{HC}$ domains. Cooperation and trust between parents, teachers and $\mathrm{HC}$ professionals can be improved to benefit the youngsters, but sometimes medical confidentiality is an obstacle. Both the educational and $\mathrm{HC}$ system are bound to rules and regulations, which can be limiting when it comes to provide tailored support. Rigid systems and the performance-driven educational financing have a paralyzing effect and hinder innovations. To provide tailored support, educational and HC professionals mentioned that they had to bend the rigid rules.

In the focus groups, all stakeholders including parents mentioned that setting achievement expectations too high may lead to a fear of failure, whereas setting them too low may cause frustration and hamper motivation. In the Dutch educational system, several difficulty levels are already established early in secondary school, and early selection determines children's future study opportunities $(23,27)$ Selection takes place in the eighth grade of primary school when children are twelve years old, where the teacher's advice together with an aptitude test determines the secondary school level (see Box 2). This advice is lower for children of lower educated parents and for children with a non-Western background (often a combination as well) (28). Reasons suggested by the Dutch Inspectorate of Education such as teachers' lower expectations toward these children and schools' priority for a high success rate at the expense of individual children were also mentioned in the focus groups. Furthermore, parents' drive to send their child to a high educational level results in extracurricular tutoring and exam training, which is not affordable for poorer lower educated parents (28).

Not only parents are performance-driven, so are schools and society as a whole. In primary school, children are tested from the beginning, and in secondary school, excellent pupils are often being put together in master classes. Schools, performing above average, are allowed to call themselves "excellent school". In contrast, schools which are unable to comply with the performance-norm will receive less money (29), which is typical for a performance-driven system (extrinsic motivation) (30). Yet, schools are expected to include students with, for example, learning and behavioural problems. With the Dutch Act 'inclusive education', Dutch schools must provide a suitable learning place to every child, including children who need extra support. In principle, in inclusive education the possibilities and the educational needs of children are crucial, and not the limitations (31). However, despite this renewal, the Dutch educational system is very restrictive, performance-driven and holds on to rigid methods. Furthermore, most schools are poorly facilitated and far behind with technical developments (32). For personalized education, children should not be compared to other pupils, but to themselves such as in the 
Finnish educational system (33). Such a system is based on trust and responsibility, and teachers are free to adapt their education to the needs of the individual child. The Dutch educational and HC systems however, are based on management and control, which works paralyzing and against innovation and tailored support (34).

In the focus groups, educational and HC professionals mentioned that they needed to get out of their comfort-zone and bend the rigid rules, to provide sufficient support. YHC professionals, dealing with budgetary savings that limited their possibilities to reach children at risk, mentioned that they, relying on their professional judgement, rebelled and went beyond the regulations to reach the children who needed care. Prevention, including preventing youngsters from dropping out of school, should be more valued. Health and prevention are often seen as a cost instead of benefits (35). However, health prevention may decrease costs and increase social wellbeing $(36,37)$. It contributes to the participation in the labour market, and less absenteeism through sickness (38).

All stakeholders agreed that they need to cooperate for the best interest of the child. In practice, role conception and indistinct boundaries between education and $\mathrm{HC}$ regarding family problems or behaviour problems makes cooperating difficult. The concerns, mentioned in the focus groups regarding cooperation between educational and HC professionals, such as unknown with each other's work and medical confidentiality and privacy regulations hindering exchanging information, correspond to those found in other studies $(14,39)$. Much distrust about knowledge and best intentions was mentioned between educational professionals and parents. They talked a lot about each other but only little with each other. Several reasons were mentioned in the focus groups; a fear of aggression when informing parents about negative aspects of their child, and teachers' insecurity about signals of children's threatened development. Parents mentioned teachers' arrogance to be hindering the cooperation. Other studies also found difficulties in the parent-teacher relationship, especially when children were in need of extra support $(11,12)$.

Together with competence and autonomy, social relatedness determines motivation (40). In adolescence the need to belong is great and students who feel disconnected are at risk for dropping out of school (41). Students who do not fit 'the norm', such as not looking, behaving and performing as 'average' children, were rejected by peers and did not fit in the educational system. More acceptances of diversity and variation are therefore needed. In the focus groups, a positive effect of male teachers on the behaviour of hyperactive boys was mentioned. However, other studies found that the sex of the teacher has no effect on the achievement, attitudes or behaviour of boys and girls in primary school (42). HC professionals mentioned the need of aligning with parents and youngsters to provide personalized care, meaning being culturally sensitive and taking into account gender differences.

The government has enforced the basic qualification to provide youngsters with better chances on the labour market (43). However, job requirements have increased and job opportunities for low educated youngsters have decreased. Moreover, children 
without a basic qualification and thus not confirming to the norm might be stigmatized in society and result in being excluded at the labour market.

The themes we found in this study fit in Bronfenbrenner's ecological system, which emphasizes the children's development in multiple social contexts and in the interactions within these contexts. (10). However, it does not completely cover the complexity of the process of school dropout. Sameroff incorporated Bronfenbrenner's contextual model in a unified theory of development (44). He included a personal change model, which explains how individuals change over time and how their competences increase from infancy on, and a regulation model, to understand how individuals interact and transact with the environment. Furthermore, a representation model was included, because all experiences of the previous models are represented in the way individuals think about something or someone. Approaching development and the dropout process from such a broad perspective may help to understand the complexity of children's unique developmental pathways. It also clarifies that youngsters at risk of dropping out of school, need personalized care.

\section{Strengths and limitations}

We chose to conduct focus group interviews, because it produces a considerable amount of information in a fairly short time period. The interaction between participants provided insight into the way they talk about this topic with each other in daily life (18). All participants were able to share their opinion and felt free to speak, as shown by a variation of opinions. Relevant stakeholders and a large diversity in experience and professions were included in this study. Most participants have children themselves, which provides them multiple roles, not only as a stakeholder, but also as a parent.

Some limitations need to be mentioned. First, we could only conduct one focus group with parents and selection bias may have occurred as only parents with youngsters who re-entered school later on, participated. Second, the focus groups are conducted and analysed in Dutch, some nuances may therefore be lost in the English text (18). Third, this study is conducted in the Dutch context and the generalizability to other countries is therefore unknown and subject for further research.

\section{Implications for research and practice}

According to the participants, coping with responsibilities and parental expectations regarding children's school performance are important determinants in the school dropout process. Since we did not measure these determinants in our previous study (16), further research for their contribution to school dropout is needed.

In this study we conducted homogeneous focus groups, but in a future study developing preventive school-dropout interventions, heterogeneous focus groups with stakeholders from education and $\mathrm{HC}$ and with parents might be valuable, especially since cooperation appeared to be so important. This will gather insight from a multi stakeholder perspective and enhance mutual understanding (18). 
This study provides recommendations and ingredients for developing early interventions to prevent school dropout. Interventions should not only target the individual child and his or her family, but also the educational and HC system. The YHC physicians, in their central role between parents, medical and educational professionals, may facilitate better cooperation between all stakeholders including parents as a linking pin. To motivate youngsters, at school there should also be attention for responsibilities, diversity and exclusion and hence for autonomy and belongingness. Furthermore, the government may create conditions for lower educated job opportunities. The educational financing should be reconsidered; the Dutch government has created a contradiction in demanding inclusive education and tailored care, but paying when complying with the performance-norm (29). Both educational and HC professionals must be facilitated sufficiently, so they can provide tailored support without having to bend the rules.

\section{Conclusion}

Beyond empowering the individual child, his or her proximal and distal environment should be included in preventing school dropout. Mutual trust between parents, school and $\mathrm{HC}$ must be restored for a better cooperation for the sake of the child and the YHC physician may be a linking pin. Educational and HC systems need to be facilitated sufficiently and be more flexible, so that professionals can provide tailored support with respect for diversity. This study will contribute to the future development of interventions to prevent school dropout at an as early as possible stage.

\section{Acknowledgements}

We wish to thank all participants of the focus groups. Furthermore we would like to thank all colleagues from the YHC division of the GGD Brabant South-East, for helping us recruiting participants for the focus groups. 


\section{References}

1. Freudenberg N, Ruglis J. Reframing school dropout as a public health issue. Prev Chronic Dis. 2007 Oct;4(4):1-11.

2. De Witte K, Cabus S, Thyssen G, Groot W, van den Brink HM. A critical review of the literature on school dropout. Educational Research Review. 2013;10(0):13-28.

3. Breslau J. Health in Childhood and adolescence and High School Dropout: University of California, Davis2010.

4. The Ministry of EducationCulture and Science. The approach to Early School Leaving. The Hague, NetherlandsMarch 2014. Available from: http://www.aanvalopschooluitval.nl/userfiles/file/2014/VSVBoekje_UK_2014.pdf.

5. Theunissen M-J, Bosma H, Verdonk P, Feron F. Why Wait? Early Determinants of School Dropout in Preventive Pediatric Primary Care. PLoS One. 2015;10(11):e0142315.

6. Theunissen M-J, de Man I, Verdonk P, Bosma H, Feron F. Are Barbie and Ken too cool for school? A casecontrol study on the relation between gender and dropout. The European Journal of Public Health. 2015;25(1):57-62.

7. Bosma H, Theunissen M-J, Verdonk P, Feron F. Low control beliefs in relation to school dropout and poor health: findings from the SIODO case-control study. BMC Public Health. 2014;14(1):1237.

8. Bridgeland JM. The new dropout challenge: Bridging gaps among students, parents, and teachers. New Directions for Youth Development. [Article]. 2010 Fall2010;2010(127):101-10.

9. Bridgeland JM, Dilulio Jr JJ, Morison KB. The silent epidemic: Perspectives of high school dropouts. Civic Enterprises. 2006.

10. Bronfenbrenner $U$, Morris P. The ecology of developmental processes. In: Damon, editor. Handbook of child psychology. Hoboken, NJ: Wiley; 1998. p. 993-1028.

11. Sandberg A, Ottosson L. Preschool teachers', other professionals', and parental concerns on cooperation in preschool - all around children in need of special support: the Swedish perspective. International Journal of Inclusive Education. 2010 2010/12/01;14(8):741-54.

12. Savas AC. The Contribution of School-Family Cooperation on Effective Classroom Management in Early Childhood Education. Kuram Ve Uygulamada Egitim Bilimleri. 2012 Fal;12(4):3099-110.

13. Osterman KF. Students' Need for Belonging in the School Community. Review of Educational Research. 2000 September 1, 2000;70(3):323-67.

14. Smeets $E$, Wester M. Knelpunten in de samenwerking tussen onderwijs- en zorginstellingen. Den Haag: Ministerie van OCW2009 mei.

15. Krueger R, Casey M. Focus Groups, 3rd. Sage Publications, Thousand Oaks, CA; 2000.

16. Theunissen M-J, Griensven van I, Verdonk P, Feron F, Bosma H. The early identification of risk factors on the pathway to school dropout in the SIODO study: a sequential mixed-methods study. BMC Public Health. 2012;12(1):1033.

17. Wong L. Focus group discussion: a tool for health and medical research. Singapore Medical Journal. 2008;49(3):256-61.

18. Green J, Thorogood N. Qualitative Methods for Health Research. 2 ed. Los Angeles: Sage Publications; 2009.

19. Wieske RC, Nijnuis MG, Carmiggelt BC, Wagenaar-Fischer MM, Boere-Boonekamp MM. Preventive youth health care in 11 European countries: an exploratory analysis. International Journal of Public Health. 2012;57(3):637-41.

20. Braun V, Clarke V. Using thematic analysis in psychology. Qualitative Research in Psychology. 2006;3(2):77-101.

21. Polit J, Beck C. Nursing research. 8 ed. New Delhi: Williams and Wilkins; 2008.

22. Strauss A, Corbin J. Basics of qualitative research: Grounded theory procedures and techniques: Newbury Park, California: SAGE Publications; 1990. 
23. EP-Nuffic. Het onderwijssysteem van Nederland beschreven [The Dutch educational system]. 2014 [updated December; cited 2015 23-11]; 4:[Available from: https://www.nuffic.nl/bibliotheek/onderwijs systeem-nederland.pdf.

24. Nuffic. Grading systems in the Netherlands, the United States and the United Kingdom. [http://www.nuffic.nl/bibliotheek/grading-systems-in-the-netherlands-the-united-states-and-the-unitedkingdom.pdf] The Hague, the Netherlands2013 [updated July; cited 2013 10-10]; Available from: http://www.nuffic.nl/bibliotheek/grading-systems-in-the-netherlands-the-united-states-and-the-unitedkingdom.pdf.

25. European Council. Council Conclusions of 5 May 2003 on Reference Levels of European Average Performance in Education and Training (Benchmarks). Official Journal of the European Union. 2003;46(C 134/03):3-4.

26. United Nations Educational Scientific and Cultural Organization (UNESCO). The revised International Standard Classification of Education (ISCED 2011). Paris 5 September 2011.

27. Nuffic E-. Het Amerikaanse onderwijssysteem beschreven en vergeleken met het Nederlandse [The American educational system compared with the Dutch one]. 2015 [cited 2015 23-11-2015]; 2:[Available from: https://www.epnuffic.nl/documentatie/publicaties/onderwijssysteem-verenigde-staten.pdf.

28. Inspectie van het Onderwijs. De staat van het onderwijs. Onderwijs verslag 2014/2015. 2016.

29. Ministery of Education Culture and Science. Prestatiesubsidie [Achievement grant]. 2016 [cited $201627-$ 04]; Available from: http://www.aanvalopschooluitval.nl/beleid/vsv-aanpak-2012-2015/prestatiesubsidie.

30. Dweck CS. The remarkable reach of growth mind-sets Scientific American Mind. [Article]. 2016;27(1):36-41.

31. Netherlands Youth Institute. Inclusive education: A suitable learning place for every Dutch child. 2013 [cited 2015 15-11]; Available from: [http://www.youthpolicy.nl/yp/downloadsyp/Inclusive-education.pdf]

32. Selwyn N. School 2.0: Rethinking the future of schools in the digital age. Research on e-Learning and ICT in Education: Springer; 2012. p. 3-16.

33. Ministry of Education and Culture. Finnish education in a nutshell. 2012 [cited 2016 27-04]; Available from: http://www.oph.fi/download/146428_Finnish_Education_in_a_Nutshell.pdf.

34. Sohier R. Zorg voor jeugdigen ont-regeld! Reflectie op het jeugdbeleid en visie op de toekomst. $s^{\prime}-$ Hertogenbosch: PRVMZ 2008.

35. Van der Lucht F, Polder J, Post N, Zwakhals L. Gezondheidszorg is niet alleen een kostenpost. Me Judice. 201022 oktober 2010.

36. McKee M, Suhrcke M, Nolte E, Lessof S, Figueras J, Duran A. Health systems, health, and wealth: a European perspective. Lancet. 200924 januar 2009;373(9660):349-51.

37. Post N, Zwakhals S, Polder J. Maatschappelijke baten - Deelrapport van de Volksgezondheid Toekomst Verkenning 2010 'Van gezond naar beter'. Bilthoven: RIVM2010.

38. Schultz A, Chen C, Edington D. The cost and impact of health conditions on presenteeism to employers: a review of the literature. Pharmacoeconomics. 2009;27:365-78.

39. Smeets E. Samenwerking tussen primair onderwijs, voortgezet onderwijs, regionale expertisecentra en jeugdzorg. 2007.

40. Ryan RM, Deci EL. Self-determination theory and the facilitation of intrinsic motivation, social development, and well-being. American Psychologist. 2000;55(1):68-78.

41. Brown MR, Higgins K, Paulsen K. Adolescent Alienation: What Is It and What Can Educators Do About It? Intervention in School and Clinic. 2003 September 1, 2003;39(1):3-9.

42. Driessen G. The Feminization of Primary Education: Effects of Teachers' Sex on Pupil Achievement, Attitudes and Behaviour. International Review of Education. [journal article]. 2007;53(2):183-203.

43. Herweijer L. Gestruikeld voor de start. De school verlaten zonder startkwalificatie. Den Haag: Sociaal en Cultureel Planbureau2008 26 Augustus.

44. Sameroff A. A Unified Theory of Development: A Dialectic Integration of Nature and Nurture. Child Development. 2010;81(1):6-22. 



\section{Chapter 8}

\section{General discussion}





\section{Introduction}

Dropping out of school is a complex educational and a health problem which affects individual youngsters, their families and society as a whole. Yet, school dropout is heavily understudied from a public health perspective. Furthermore, rather than acting upon the early life, most interventions to reduce school dropout are targeted toward monitoring and, by occurring late in the dropout process, many are foremost repressive (1). The aim of this thesis was to identify early biopsychosocial determinants in the pathways to school dropout. Furthermore, we aimed to gain an in-depth understanding into these pathways from the perspectives of the young adults, parents, and educational and health care professionals. Such understanding will contribute to the future development of a tool for the youth health care (YHC) system to identify children that are at risk of dropping out of school at an early age.

In this thesis, the following research questions are addressed:

1. What biopsychosocial determinants in infancy, early and middle childhood, and adolescence predict school drop-out in young adulthood (18-23 years)?

2. Are gender beliefs and self-reported masculinity and femininity, beyond the influences of sex, related to school dropout in young adulthood?

3. Are adolescent low control beliefs, independent of parental socioeconomic background, related to school dropout and poor health in young adulthood?

4. How do young adults who graduated with a delay or dropped out of school perceive their life history and particularly their time in school?

5. How do parents, stakeholders from education and stakeholders from health care view the reasons for dropping out of school and their own and each other's roles in preventing school dropout at an early age?

In this chapter, I give a brief overview of the study design, integrate the quantitative and the qualitative findings of the studies, and provide some reflections on the findings and the methods used. Furthermore, I present implications for future research, practice, and policy.

\section{Methods}

The Stay in or drop out (SIODO) study is based on a sequential mixed-methods design. First, a quantitative case-control study with data from the YHC-files and an additional questionnaire was conducted as described in chapter 1 . The life course data from the YHC-files were collected in the everyday practice of the YHC professionals from birth 
onwards, in order to examine the biopsychosocial determinants from a life course perspective. The results of the case-control study were input for a qualitative study with semi-structured individual interviews with 25 young adults (22-25 years). These interviews helped us to understand the previous case-control findings and provided insight in the processes to school dropout from the perspectives of the young adults. Subsequently we conducted five homogenous focus groups with parents, stakeholders from education and stakeholders from YHC respectively. In the focus groups, participants reflected on the results of the case-control study and the interviews, and discussed their own view on dropping out of school. Furthermore, findings from the previous focus groups were also input for the following focus group sessions (Figure 1). The successive studies were complementary and increased our understanding of the pathways to school dropout.

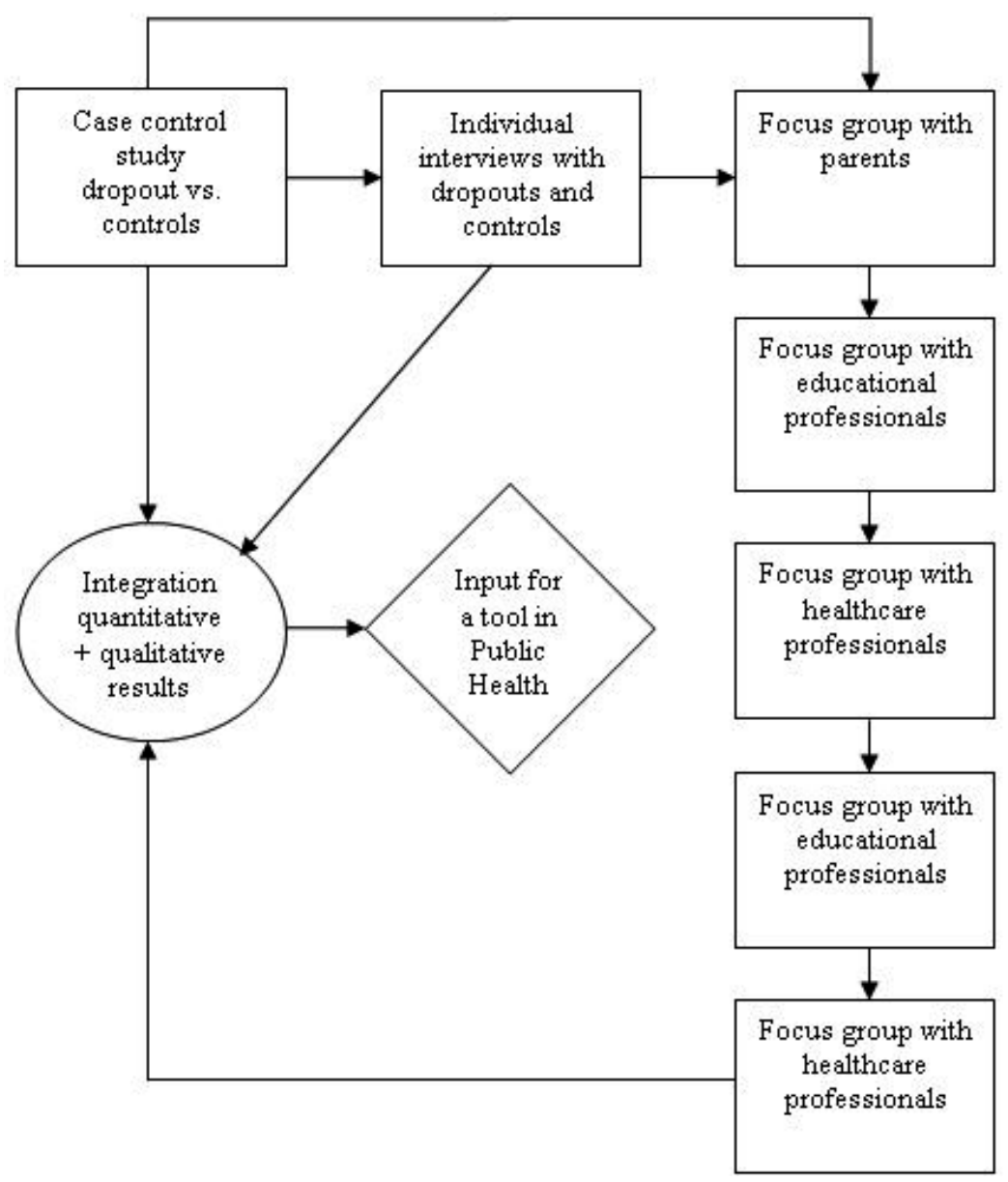

Figure 1. The SIODO study design 


\section{Main research findings}

Biopsychosocial risk factors and protective factors on the pathways to school dropout from birth onwards were identified. Youngsters from one parent families, with a nonWestern background and living in areas with cheaper houses were at greater risk of dropping out of school. Moreover, these youngsters more often missed one or more programmed preventive health examinations from the YHC, which further increased the risk of school dropout. According to parents, as mentioned in the focus group, it is not always clear what the $\mathrm{YHC}$ has to offer.

At all age categories, poor relationships between parents were associated with later school dropout (18-23 years). According to interviewees, parents' fights were mostly about a lack of money, and fights heavily impacted the children. Domestic violence, including physical and mental child abuse, could lead to decreased self-esteem and attention problems at school. At all age levels, an accumulation of stressful life events was associated with school dropout.

The birth of a sibling in the 0-4 age period protected against later school dropout. According to parents, this might be due to the responsibility given to children for being the 'older one' and a role model, which resonated with some interviewees reflections how they wanted to be a role model for a younger brother or sister. Educational and care professionals mentioned that children must learn how to take responsibility for their own learning process and that parents and teachers should allow children to take that responsibility, appropriate for their age. When expectations about learning achievements do not match the children's developmental level, for instance when these expectations were set too high or too low, this could lead to fear of failure and decrease self-confidence. Children with an insufficient hearing test in infancy (0-4 years) were at higher risk for school dropout, but also for learning problems at the age of 4-8 years, which in turn increased the risk for dropping out of school in young adulthood. Having experienced hospitalization or surgery, including treatment of hearing impairment with tympanic drains, in the 4-8 age period decreased the risk of dropping out of school. Children aged 4-8 years, who did not participate in sports, were at higher risk for school dropout. Children with learning difficulties in this age group more often exhibited externalizing behaviours at the age of 8-12 years, which was also associated with dropping out of school.

Some interviewees mentioned being bullied at primary school (4-12 age period), which decreased self-esteem and pleasure in school. At secondary school (12-16 years) bullying was mentioned as a reason for school absenteeism, which in turn was strongly related to school dropout. Stakeholders from education and care mentioned that adolescents do not want to differ from the norm, meaning that they want to belong to a peer group and do not want to perform better or worse than their class mates. They also mentioned that there is little space for diversity among peers but also in the school system. Highly masculine girls and feminine boys, who simultaneously considered 
themselves hardly masculine, were at higher risk of dropping out of school and so were highly masculine boys with strong normative beliefs about male peer relationships. Exclusion from a peer group was often accompanied by bullying and violence. Girls with an early puberty (measured by menarche age) and girls who got pregnant in their teens also were at higher risk for school dropout.

Having a sense of self-efficacy and mastery in adolescence decreased the risk of dropping out of school and interviewees, reporting having a sense of control beliefs and feeling self-confident were more likely to have finished an education. Feeling connected to family, peers and teachers and experiencing support and understanding were reported to be important for a successful school career. However, to provide tailored support, professionals from education and care mentioned that they had to step out of their comfort zone and bend the rigid rules and regulations of the educational and health care systems. Cooperation and trust between parents, teachers and care professionals can be improved to benefit the youngsters, but sometimes medical confidentiality is an obstacle. Parents mentioned confidentiality to be important for a trustful relationship with care professionals. Teachers however, thought that care professionals were hiding behind privacy rules and therefore did not share the acquired information for providing good care. Care professionals in turn, mentioned that they were bound by professional secrecy. According to some interviewees, professionals did neither listen nor took action on their signs of unsafety and professionals mentioned that they wanted to be absolutely sure about the signs of family problems before they could take action. Moreover, some educational professionals mentioned that they find it difficult to talk to parents about their worries, or even questioned whether they were allowed to interfere with family problems when school results were sufficient. Nevertheless, if a youngster needs support, the whole family should be involved in the guidance. In the next section I will reflect on these findings.

\section{Reflection on the main findings}

This section is outlined as follows. First, I reflect on the ICF-CY model, used to categorize the many determinants of the YHC files and the developmental background of school dropout. Then, I discuss the importance to grow up in a safe environment. Subsequently, I discuss the necessity to meet the "norm" in today's society and finally I reflect on gender differences and socioeconomic health differences.

\section{The functioning and participation of children.}

In this thesis, the biopsychosocial model was used as a basic theoretical framework and the international classification of functioning, disability and health for children and youth (ICF-CY), based on this model, was used to categorize the determinants from the 
YHC-files (2). The ICF-CY assesses children's functioning and their participation at the level of the whole human being, in day-to-day life (3). Quality of life and functioning are central, rather than having a disease or a DSM diagnosis and the ICF-CY therefore aligns with Huber's concept of positive health. However, according to Huber the ICF lacks a classification for personal factors. Huber defines health as "the ability to adapt and to self-manage in the light of the physical, emotional and social challenges of life" (4). She developed the "spider web" with six dimensions of positive health, which can be seen as a conversation tool and as a diagram for people to score their own health perceptions (Figure 2). Furthermore, it may be input to classify personal factors in the ICF. According to Huber, resilience and having a sense of control beliefs are resources for positive health. The young adults who dropped out of school however, reported lower levels of mastery and self-efficacy. Moreover, they more often used non-productive coping strategies such as withdrawal. Adapting to the physical, emotional and social difficulties they faced was hard for them. In this light, "adverse health" must be perceived as predictive for dropping out of school.

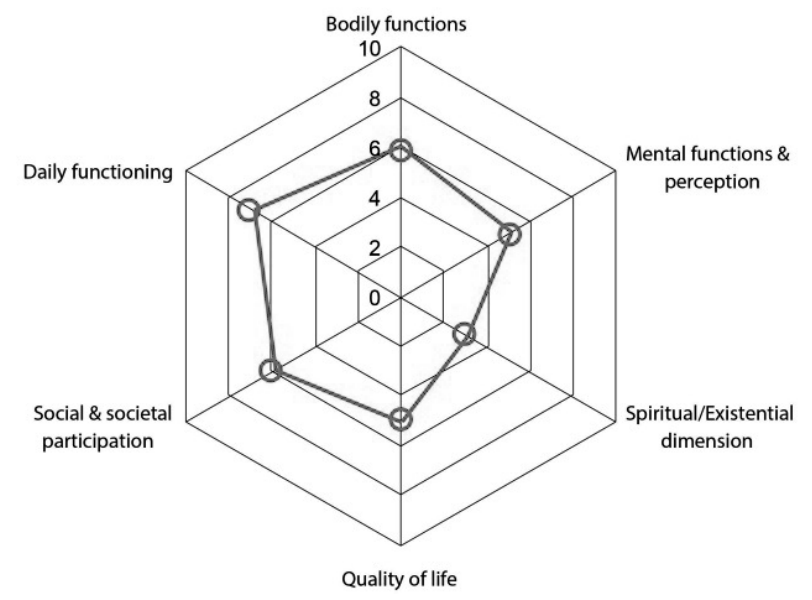

Figure 2. The "Spider web" with the six dimensions of positive health.

From: Huber M, van Vliet M, Giezenberg M, et al. Towards a 'patient-centred' operationalisation of the new dynamic concept of health: a mixed methods study. BMJ Open 2016;5:e010091. doi:10.1136/bmjopen-2015010091

The ICF-CY is a classification model, and the determinants related to school dropout covered all domains. However, we noticed that at different ages, different determinants played a role.

In infancy (0-4 years of age) the emphasis was on the environmental determinants, whereas in adolescence (12-16 years of age) most factors, significantly related to school dropout could be categorized into the domains "personal factors" and "participation". This was to be expected, as the younger the children are, the more dependent they are 
of their environment. The balance between other-regulation and self-regulation shifts when children can take on responsibility for their own well-being (5).

Dropping out of school is not a discrete event, but a dynamic process $(6,7)$, in which not only the biopsychosocial factors play a role, but also the interplay between the child and his or her proximal and distal environment. Furthermore, the way parents, school and health care work together for the sake of the child influences the dropout process (8). In his unified theory of development $(9,10)$, Sameroff combined four different models; a personal change model, which explains how individuals change over time and how their competences increase from infancy on; a contextual model, explaining how all individuals are influenced by their environment; a regulation model, to understand how individuals interact and transact with the context, and a representation model, because all experiences of the previous models are represented in the way individuals think about something or someone. Later, the evolutionary model was added, because the context (people, the developmental system, the world) also changes over time. Approaching development from such a broad perspective may also help to understand the complexity of school dropout. It should be clear that youngsters can only be helped with tailored care. Moreover, the entire system must be taken into account because everything affects or is affected by something else (5).

\section{Growing into school dropout}

Development is a gradual process and every child has its own unique pathway. Complex interactions of many determinants result into a countless number of unique pathways which lead to an infinite variability of unique individuals. Among the many determinants are factors such as genetic background, environmental influences, lifestyle, and social relations and received support. Therefore, a "one size fits all" approach in the YHC, which has proven its suitability earlier and in particular for screening programs such as neonatal screening (11-13), requires an eye for more complexity to tackle problems such as school dropout. Time has come for differentiation and tailored care.

The YHC aims to signal and prevent or "treat" as early as possible the symptoms that hinder children's daily functioning, and enable them to continue their own optimal pathway. This may prevent problems to end up in heavy specialist care or in early school leaving, in case of symptoms related to school dropout. Furthermore it may prevent labeling with a diagnosis and stigmatization. Care must be taken that signaling emerging physical and mental health problems at an early stage will not medicalize children's problems, but rather helps to prevent health problems to become social problems such as school dropout and unemployment, and vice versa, helps to prevent social problems from becoming health problems.

Children grow into school dropout when they do not have enough resilience to cope with their vulnerabilities (14). In this study, the biopsychosocial risk factors identified are vulnerabilities related to school dropout. Youngsters were particularly vulnerable 
when they felt socially disconnected from persons who were important to them, such as family and peers, and when they grew up in an unsafe environment. In the interview study, the relevance of including the environmental context into the explanation and prevention of dropping out of school became clear. Both children and their families should be empowered (increasing the strengths and resilience), and a safe environment is required for the entire family, and in school, for all children. The YHC can play a role to ensure such an environment.

\section{Care for a safe environment of the child.}

In 2010, 3\% of all children in the Netherlands have been emotionally, physically, or sexually abused and this is considered to be under-reported $(15,16)$. Yet, in the YHC files, child abuse was recorded even less. This might be due to the way the health examinations were organized with questionnaires and consultations based on checklists. Adolescents in the interview study mentioned that they did not report sensitive issues on a questionnaire, particularly if they did not know who was going to read it. Thus, in case of face-to-face contacts, the YHC professionals should go beyond the questionnaires and checklists and enter into a dialogue with parents and youngsters. Furthermore, both parents and children may avoid health examinations in case of domestic violence and child abuse (16). In this study, child abuse was related to school dropout and to missing one or more health examinations of the YHC. Furthermore, children who missed one or more health examinations had higher odds of being abused as well as of dropping out of school. These children are thus at higher risk for deflecting developmental pathways and need to be monitored.

Some interviewees mentioned that they did report their abuse at school, but did not feel taken seriously. In the focus group interviews, some teachers believed that they were not allowed to interfere with family problems, unless the school results were decreasing. Other teachers, but also health care professionals, felt concerned but found it important to be absolutely sure about the signals and kept collecting "evidence" before contacting parents and reporting the suspected abuse. Some professionals felt afraid to damage their relationship with parents. However, when there is no transparency and trust, the relationship is already damaged and waiting to see what was happening resulted in ongoing abuse and the child's loss of faith in support. Therefore, both educational and health care professionals need to step out of their comfort zone and discuss their concerns with the parents as well as the youngsters themselves.

\section{Meeting the norm.}

Living up to the norm seemed to be important to meet the expectations of others and to fit into "the system". Many schools still use a 'one size fits all' approach, where children who need to be challenged get bored whereas children with learning difficulties 
cannot come along with the rest of the class. Some children are late bloomers or cannot listen and sit still for a long time, and all children develop in their own unique way. Labeling children as "abnormal" however is an artefact of the normal distribution and not a characteristic of children themselves (9). Some primary schools act on children's talents, and do not speak about 'learning problems' because every child follows its own developmental pathway. Some children follow a different path or are better at learning "on the job". Yet, there is a normative belief that it is best for all adolescents to go to school. However, for some students, dropping out of school may be a solution to their problems rather than being a problem in itself, and staying in school may harm their self-esteem and self-confidence. Other students may have other priorities for the time being or are financially forced to go to work. For some youngsters, their cultural or socio-economic background may clash with the white middle-class culture in the school system, and some researchers believe that education aims at creating and selecting obedient people who are to prepared to function well in a middle-class oriented society $(17,18)$. In the traditional educational system, variation must be kept small and there is little space for youngsters' authenticity. Moreover, by setting the norm that everyone has to have a basic qualification, we have also created a problem group, namely the youngsters without one. By not conforming to the norm of having a basic qualification they run the risk of being excluded for a job and stigmatized in society. However, in this study some youngsters did manage well in society without a basic qualification, although they had experienced major adversities in childhood. By focusing on staying in school and only considering dropout as the problem itself, we may offer a symptomatic treatment rather than targeting a cause. Therefore, it is more important to understand why youngsters no longer want to go to school, and what is going on in their lives. By focusing on such underlying problems, youngsters' lives may become a lot more pleasant, and thus, have a higher quality.

Children often had a hard time in school when they deviated from the norm, such as being smarter than most kids, being poor, wearing different clothes, having a different ethnic background, or not identifying as heterosexual. Especially at secondary school, when youngsters are most vulnerable to peer pressure, they risked being excluded when they did not meet the criteria of being "cool" (19). Interviewees mentioned that good looking boys and girls with a 'big mouth' were most popular among peers. Having 'guts' provided status amongst boys, and also for girls, although they had to be additionally 'girlish'. Some of the tougher, more laddish girls became "too cool for school" by connecting with deviant peers in a hang group and started smoking pot, drinking alcohol, and playing truant. However, in special education and in a classroom with a lot of diversity where everyone deviated from the norm, differences were more acceptable. Therefore, the margins of the norm need to be stretched and diversity and accepting difference needs to be open for discussion with all students. For teachers, this requires being open to the stories of others and being aware of their own norms and 
backgrounds and how these play a role in their teaching and relationships (selfreflection and reflexivity).

\section{Measuring gender}

From an early age on, expected norms and gender roles differ for boys and girls. Girls are expected to behave more girlish, do their homework diligently and be obedient. Boys are found to be cuter when they are rebellious and expose outgoing behavior as a preschool kid. However, as they grow older, rebellious behavior is not as acceptable anymore, and conflicts between students and teachers may arise. There may be a discrepancy between how feminine and masculine youngsters feel about themselves and what their ideals are about men and women. Gender differences and the environmental expectations are complicated and so is measuring the concept of gender. Considering a youngster as either a boy or a girl rather than for instance as having both masculine and feminine typed characteristics or adhering more or less to gendered beliefs, is inadequate and excludes all gender nuances and possible fluidities.

In this study, both male and female young adults were asked to rate their femininity and masculinity (at the age of 16 years). Furthermore, they were asked to respond to: (1) 12 belief statements about appropriate behaviour for males into interpersonal relationships among peers (AMIRS questionnaire) (20), and (2) 10 statements about conventional femininity ideologies of inauthenticity in relationships (based on the ISR scale of the AFIS questionnaire) (21). The AMIRS questionnaire is developed for male and the AFIS for female adolescents. By our best knowledge, this is the first study that uses these questionnaires for both, male and female young adults. It is interesting and relevant to use the AMIRS for young women too, as it measures the assumption that a man is ideally not vulnerable and if he is, he should not show it. It says something about how young women look upon the male gender role. The AFIS on the other hand, measures how youngsters would react in a friendly relationship. More often for girls the norm is to be friendly and always try not to hurt a friend's feelings, which manifests in girls having to constantly work on themselves. Using the AFIS with young men, may therefore be indicative for internalizing behavior. Furthermore, by using all questionnaires for male and female young adults we created different interaction possibilities, namely between self-rated femininity/masculinity and gender beliefs (AMIRS/AFIS).

The study showed that the relationship between gender (self-perception and gender beliefs) and school dropout differed for female and male young adults. The effect for young women turned out to be U-shaped, as an intermediate score on the gender variables tended to protect best against dropout. An explanation might be that having both more masculine and feminine typed traits increases the number of coping options (22, 23). For young men however, a gender effect presented itself in terms of interactions. Male adolescents, who find themselves highly masculine, are not at higher risk for dropping out of school. After all, masculinity is considered the norm for men in our 
society. However, when highly masculine young men simultaneously held strong hegemonic beliefs about how a men should behave in peer relationships, they risked being "too cool for school". Understanding how gender influences behavior, and consequently the pathways to school dropout, may help us to unravel these processes and create openness to discuss norms and values. Furthermore, by departing from a binary, dichotomous view of gender (male/female) and providing children with the opportunity to rate their feminine and masculine traits in a sliding scale, shades of gray were added to the 'normal range' of opportunities which will decrease the stigma and the feelings of being different. Having that said, terminology such as 'masculine or feminine typed' or 'male and female characteristics' is problematic in itself and may contribute to the very same stereotyping that it aims to deconstruct. This issue however is beyond the scope of this dissertation.

\section{School dropout and socioeconomic health differences.}

Parents' educational, occupational and income level are a strong predictor of their child's academic achievement. Children growing up in a family with a low socioeconomic position are at higher risk of dropping out of school (24). School dropout in turn, reduces the chance of finding a job and without a job and depending on welfare the status quo will be maintained and one will end up in a lower SES (25). Low SES is associated with poorer health, and in this study school dropout and poor health were found to be strongly related. As explained in chapter 1, there are different hypotheses about the origin of socioeconomic health differences. In this thesis insight was gained into how the direct and indirect selection mechanism played a role. First, we found evidence for the indirect selection mechanism, as low control beliefs seemed to be an important predictor for both health complaints and school dropout, regardless of socioeconomic background. Nevertheless, experiencing little control over your own situation or the environment is connected to a low educational and income level (26). People with a low income and less job autonomy experience less choice opportunities, which may lead to fatalism, disempowerment, and poorer health $(27,28)$. However, some people in a lower socioeconomic situation do manage to maintain good physical health. They find positive role models, early in life, who teach them to have a positive view of others and trust and to depend on others in life. In this way, they are able to develop a shift-andpersist approach to life that balances shifting oneself by accepting and adapting to life's day-to-day demands with persisting in life through endurance and holding on to meaning and optimism (29). Those children are more resilient, better able to regulate their emotions and are more focused on their goals in the future. In line with this, a higher level of self-efficacy, feeling socially connected and having a role model were found to be protective for school dropout. Furthermore, according to Bandura's self-efficacy theory, personal efficacy expectations determine whether coping behavior will be initiated, how much effort is expended, and how long it will be sustained in the face of 
stressful events (30). This provides an opportunity for the YHC to contribute at an early age to reducing early school leaving and socio-economic health differences later in life.

In the literature, SES is seen as an important topic related to school dropout, but the youngsters in this study do not explicitly experience this relation. For children who grew up in poverty or in a poor neighborhood a low SES is the norm. As mentioned before, youngsters who dropped out of school more often lived in a poor neighborhood and missed one or more health examinations of the YHC. Families that do not show up at a YHC's health examination often face difficulties in several areas, such as financial difficulties and severe parenting problems (31). Therefore, parents can easily forget an appointment or avoid going to the YHC, in case they feel ashamed or guilty or when they are mistakenly afraid that their children will be placed in a foster family (31). In addition, some parents shirk the YHC examinations, because they do not have the time or inclination to come or because they do not see its relevance. When their cultural background, norms, and views do not correspond to those of the current dominant white middle class, they may find it even harder to connect to its institutions, and care organizations can be hard-to-reach. To bridge this gap, the YHC should increase her accessibility (31). Some families can best be reached by phone, or with a home visit. For other families, a conversation together with an interpreter may be helpful. Therefore, the YHC has to find suitable ways to approach hard-to-reach groups and in particular when these groups consists of non-Western one-parent families with a low socioeconomic status

\section{Methodological considerations}

School dropout is a dynamic process, however, we measured school dropout in a certain period namely during the school year 2010-11. Some students who dropped out of school returned to school later on to finish an education. During this study, a number of laws and regulations have come into force, such as the participation Act (January 1st 2015), which forces young adults under 27 years of age without a basic qualification and without a job to return to school, because they are not entitled to receive social welfare anymore (32). Moreover, many interventions had taken place to reduce school dropout, although they were all carried out late in the dropout process (1).

\section{Strengths}

This study has several strengths. To develop a more complete understanding of the complex process of school dropout, an explanatory sequential mixed methods design was used, in which qualitative studies built on quantitative studies. The rationale for using such a design was that quantitative studies (case-control studies) would provide a general understanding of determinants related to school dropout, and that qualitative studies (semi-structured interviews and homogeneous focus groups) could help to un- 
derstand underlying mechanisms and explore participants' views in more depth (33, 34). Both methods complement each other, and take advantage of each other's strengths $(35,36)$. Another strength to the study is that the integration of the quantitative and qualitative methods not only took place at the design level (explanatory sequential), but also at the methods level. For instance, the quantitative data base (1) linked to the interview study through purposive sampling by selecting the interview participants from the respondents in the case-control study (connecting), and (2) informed the data collection approach of the qualitative studies via the interview guides (building). In chapters 3 to 7 of this thesis, results of the case-control studies, the interview study and the focus group study are separately reported. In this chapter, findings of all studies are integrated in a narrative by making use of weaving in which we connect the results to each other theme-by-theme (37). Regarding the fit, the qualitative findings mostly confirmed the quantitative findings and increased our understanding of the pathways toward school dropout.

A third strength to this study is the effort to prevent bias as much as possible. To avoid selection bias, the control group was highly similar to the cases at the start of the school year 2010-11. Both cases and controls had not yet attained their basic qualification at the age of 18-23 but were still attending school. Simultaneously, this might have led to an underestimation of associations between determinants with school dropout compared to a study in which all possible controls and cases are included. To avoid recall bias, previous collected information from the YHC-files was used.

\section{Limitations}

Some limitations need to be addressed. First, the information from the YHC-files was not biased, but in the questionnaire and in the interviews we asked the young adults about their childhood. Therefore, recall bias may have taken place, and memories may have been influenced by events in later life such as school dropout itself. Second, as we had expected the cases to be a hard-to-reach group, we tried everything to get a sufficient response. We announced this study in free local newspapers, hung posters in community centers where 18-23 year old youngsters spent time and we placed information cards at the municipal compulsory education department and at social services, where these young adults registered for guidance to education and work, and for social welfare. Respondents had a chance of 1 to 10 to win a cinema ticket. Nevertheless, the non-response rate was substantial and despite all our efforts, the high-risk groups are missing in our study. We had to send 8,630 questionnaires to recruit 1049 possible participants (12 percent), under which 330 cases. Not only young adults were hard to reach, but their parents were as well. Therefore, we had to limit ourselves to one instead of two focus groups with parents. For both participants and non-participants, we collected data on dropout status, age, sex and socioeconomic status from external socioeconomic data at zip-code level. Cases (compared with controls), men, and those living in areas with cheaper houses less often filled out the questionnaire and cases, young- 
sters with a non-Western background, living in one-parent families or in areas with cheaper houses or having a lower educated mother more often missed one or more $\mathrm{YHC}$ health examinations. Hence, selection bias may have occurred and the influence of sex and socioeconomic status on school dropout may be underestimated. Third, since not all data in the YHC-files were complete, some important information may be missing. Child abuse, for example, had a low prevalence in our study and was not identified in the analysis as a risk factor despite its high OR. Possibly, child abuse is underreported in the YHC- files. Other important information missing was about teenage pregnancy and about (domestic) violence.

The study deviates from the original study protocol in chapter 2 in three ways. First, it was intended to present the results of the case-control study to the steering group and an expert group, consisting of representatives from a youth organization that provided support for school dropouts. However, due to reorganizations within the municipality and political changes, the steering group had fallen apart and representatives of the youth organization were no longer traceable. Instead, results were presented to researchers, and YHC professionals, and an expert group consisting of educational professionals and attendance officers. Furthermore, results were discussed in-depth with the interviewees and participants in the focus groups. Second, male and female interviewers were supposed to interview the youngsters. However, when a young adult responded to the invitation, he/she wanted to make an appointment quickly and it appeared impossible to arrange an interview with one of the male researchers. Both male and female interviewees were very open-hearted about sensitive topics and about their view on gender roles and sexual orientation, and honest at the member check. Therefore, it is not to be expected that a male interviewer would make any difference in the outcome. Third, for the analyses of the life course determinants from the YHC-files, we used an age-attentive life-course approach in the modeling, because this enabled us to identify determinants related to school dropout early in life. We conducted a forward and backward stepwise regression analysis with the determinants from the YHC-files that were univariately related to school dropout. The systematic way of analyzing by comparing the forward and backward findings and a cross-validation in two random halves of the sample minimized capitalization on chance.

The pathways to school dropout and the processes underneath them appeared to be very complex and a simple tool cannot be presented. However, strength to this study is that we have gained in-depth insight about the early symptoms in the pathways to school dropout, and how these symptoms are experienced by the young adults, and parents and other stakeholders. However, to use this knowledge for developing a personalized tool that can be used in the YHC, more research is needed. 


\section{Possibilities for a future tool}

The aim of this thesis was to provide insight into the life-course pathways leading toward school dropout and to contribute to the future development of more effective, personalized interventions in $\mathrm{YHC}$ aimed at reducing school dropout at an as early as possible stage. As mentioned above, one size does not fit all, and a simple tool will not cover the complexity of the processes to school dropout. Complex problems demand tailored solutions, and it will be a challenge to develop a toolkit that provides possibilities to identify determinants related to school dropout, and different interventions, which can be adapted to the individual situation. It should be clear that identification of determinants must always be followed by a dialogue with children/ youngsters and their parents.

The biopsychosocial and ecological determinants found in this study can be used to detect and monitor children who may be at risk for dropping out of school. A distinction can be made between determinants which are easy to detect at school such as behavioral problems, absenteeism, and learning difficulties and health and social determinants which can be identified in the YHC. In this respect, a good and early disclosure of signals to the YHC by school and vice versa is important. For this purpose, the ICF-CY could be further developed to a suitable registration tool for monitoring children's development as it is not only based on diagnostic criteria, but especially on biopsychosocial factors and functioning and participation. However, the ICF-CY describes the functioning of a child at a certain moment in time (as a snapshot) and does not cover the complexity of the pathways to school dropout (as a video). Therefore, regarding school dropout, the ICF-CY requires further development into a tool which monitors children's development over the life course. Outcome measures could be linked to the different domains of the ICF-CY. This allows us to follow a child's development, to decide whether an intervention is needed, and to evaluate the outcomes of such interventions.

After developing the tool, it must be validated, preferably in a longitudinal design. For successful implementation all stakeholders (youngsters, parents, education and health care) should be involved from the start.

\section{Recommendations for future research}

In addition to the development and validation of a tool, other recommendations can be made for future research. In this study, biopsychosocial risk factors and protective factors on the pathway to school dropout from birth onwards were identified. However, due to a low response rate and thus a low power and a large amount of determinants in the YHC-files, stratification for sex, ethnicity and SES was not possible. Yet, there is strong evidence that some determinants differ in various subgroups. Further research in a larger sample, with separate analyses for sex, ethnicity and SES and their intersections 
is recommended. Moreover, future studies should examine the associations with a longitudinal design including all possible cases and controls. In the questionnaires, the themes found in the qualitative studies should be included.

Gender beyond sex difference is still understudied in public health research, but our findings underscore its importance. Using the AMIRS and the AFIS questionnaires for both male and female young adults provided valuable information. Both questionnaires need to be further validated for Dutch adolescents and for both sexes. Furthermore, given the complexity of relations between gender typed traits and gendered beliefs (curvilinearity in women and interactions in men), the concept and assessment of gender also require further theorizing, refinement and research.

Low control beliefs were found to be an important risk factor for dropping out of school and for poor health. Among other things, future research should examine how those low control beliefs develop, how they can be detected and at what age in particular. This can provide opportunities for intervening in the YHC to increase control beliefs.

\section{Recommendations for practice and policy}

School dropout is a public health problem, and from January 1, 2015, identifying and preventing absenteeism and early school leaving is included in the YHC-package (38). Municipalities are responsible for ensuring that the YHC- package is available and actively offered to all children and adolescents up to 18 years. This thesis can contribute to signaling a heightened risk of school dropout already at an early stage and to help with optimizing interventions that aim to prevent dropping out of school and eventually reduce socioeconomic health differences. Based on the findings in this thesis, the following recommendations are formulated:

\section{The individual and beyond}

Children's development is inextricably linked to its environment, which means that in case of stagnation both the child and his or her family should be empowered (by increasing strengths and resilience). Schools and the YHC can play a role in creating and maintaining a safe environment for children, free of bullying, and with the acceptance of (e.g. ethnic and sexual) diversity. Furthermore, school and the YHC should be aware of signals of child abuse and discuss them with parents. For children's self-confidence it is important that the responsibilities they have to carry match their capabilities, because when expectations are set too high this may lead to a fear of failure. In case of low control beliefs, children's level of self-efficacy and self-esteem must be increased as especially self-efficacy is strongly related to school dropout. Most children and especially youngsters need to belong to a peer group and feel socially connected to and supported by their family. School and the YHC should be aware of this and when proper 
social binding is lacking, they can take measures to promote social connectedness with parents, peers and teachers.

\section{Building a bridge}

Cooperation between education, YHC and parents (and youngsters) is necessary to tackle complex problems such as school dropout for which mutual respect is required. To enhance mutual trust and not to be hampered by privacy and confidentiality rules, parents must be included in the process right from the beginning. Open communication is beneficial for understanding and collaboration between all stakeholders.

\section{Adapting the procedure of the $\mathrm{YHC}$}

Signaling risks starts with an adequate registration, not only at school (absenteeism) but also in the YHC. The ICF-CY may be helpful with this and paying attention to the personal factors, the spider web of "positive health" can additionally be used to cover meaning and self-perception of health (4).

Furthermore, to collect information on sensitive topics, it is important to connect to parents and youngsters, to go beyond the questionnaires and checklists and enter into a dialogue. Parents and youngsters should have as much control as possible, and their needs should be leading. Some families are hard-to-reach and for some families the YHC is hard-to-reach. The YHC must increase her accessibility and actively approach families who (repeatedly) do not show up on an invitation of the YHC. Time has come for personalized care, also in $\mathrm{YHC}$ !

\section{Conclusive remark}

This thesis shows that it is possible to signal heightened risks of school dropout from an early age. With a clear registration, and in cooperation with youngsters, parents, school, and other health care partners, the preventive youth health care must be able to contribute to reduce dropout rates and thus socioeconomic health differences. However, dropping out of school is a multidimensional problem, with many complex pathways leading to school dropout. It is not about the detection of individual determinants, but the understanding of a coherent whole. Therefore, a questionnaire and checklists are not sufficient. A personalized approach is needed, in which we listen to the story of the youngsters themselves. 


\section{References}

1. The Ministry of EducationCulture and Science. The approach to Early School Leaving. The Hague, NetherlandsMarch 2014. Available from: http://www.aanvalopschooluitval.nl/userfiles/file/2014/VSVBoekje_UK_2014.pdf.

2. Theunissen M-J, Griensven van I, Verdonk P, Feron F, Bosma H. The early identification of risk factors on the pathway to school dropout in the SIODO study: a sequential mixed-methods study. BMC Public Health. 2012;12(1):1033.

3. Lee AM. Using the ICF-CY to organise characteristics of children's functioning. Disability and Rehabilitation. 2011;33(7):605-16.

4. Huber M. Towards a new, dynamic concept of health: Its operationalisation and use in public health and healthcare and in evaluating health effects of food. Maastricht: Maastricht University; 2014.

5. Sameroff AJ, Fiese BH. Transactional regulation: The developmental ecology of early intervention. Handbook of early childhood intervention. 2000;2:135-59.

6. Bradshaw CP, O'Brennan LM, McNeely CA. Core competencies and the prevention of school failure and early school leaving. New Directions for Child and Adolescent Development. 2008;122:19-32.

7. Jimerson S, Egeland B, Scroufe L, Carlson B. A prospective longitudinal study of high school dropouts; Examining multiple predictors across development. J School Psychol 2000;38:525-49.

8. Bronfenbrenner $U$, Morris P. The ecology of developmental processes. In: Damon, editor. Handbook of child psychology. Hoboken, NJ: Wiley; 1998. p. 993-1028.

9. Sameroff A. A Unified Theory of Development: A Dialectic Integration of Nature and Nurture. Child Development. 2010;81(1):6-22.

10. Sameroff AJ. A dialectic integration of development for the study of psychopathology. Handbook of developmental psychopathology: Springer; 2014. p. 25-43.

11. Lanting $\mathrm{Cl}$, Rijpstra A, Breuning-Boers J, Verkerk P. Evaluatie van de neonatale hielprikscreening bij kinderen geboren in 2007: TNO Kwaliteit van Leven, Preventie en Zorg; 2008.

12. Bolhuis P. De hielprik. Bijblijven. 2008;24(6):58-64.

13. Verloove-Vanhorick S, Reijneveld S. Jeugdgezondheidszorg: meer preventie voor weinig geld. TSG. 2007;85(7):371-3.

14. Ungar M. The social ecology of resilience: Addressing contextual and cultural ambiguity of a nascent construct. American Journal of Orthopsychiatry. 2011;81(1):1-17.

15. Netherlands Youth Institute. Child abuse, the figures. 2015 [updated 2-12-2015; cited 2016 10-04]; Available from: http://www.nji.nl/Kindermishandeling-Probleemschets-Cijfers. .

16. Wagenaar-Fischer MM, Heerdink-Obenhuijsen N, Kamphuis M, de Wilde J. Directive Secondary prevention of child abuse for the Child and Youth Health Care. TNO; 2010 [updated 09-2010; cited 2016 23-04]; Available from: https://www.ncj.nl/richtlijnen/jgzrichtlijnenwebsite/details-richtlijn/?richtlijn=12\&rlpag=822.

17. Blackledge D, Hunt B. Sociological interpretations of education: Croom Helm London; 1985.

18. King R. The sociology of school organization: Routledge; 1983.

19. Hay I. Gender Self-concept Profiles of Adolescents Suspended from High School. Journal of Child Psychology and Psychiatry. 2000;41(3):345-52.

20. Chu JY, Porche MV, Tolman DL. The Adolescent Masculinity Ideology in Relationships Scale: Development and Validation of a New Measure for Boys. Men Masc. 2005;8(1):93-115.

21. Tolman DL, Porche MV. The Adolescent Femininity Ideology Scale: Development and validation of a new measure for girls. Psychol Women Q. 2000;24(4):365-76.

22. Howard JA, Hollander J. Gendered situations, gendered selves. Oxford: AltaMira Press; 2000.

23. Murray C, Greenberg MT. Children's relationship with teachers and bonds with school. An investigation of patterns and correlates in middle childhood. J Sch Psychol. 2000;38(5):423-45.

24. De Witte K, Cabus S, Thyssen G, Groot W, van den Brink HM. A critical review of the literature on school dropout. Educational Research Review. 2013;10(0):13-28. 


\section{CHAPTER 8}

25. Eurostat. Europe 2020 indicators. 2012 [updated 17/09/2012 11:20:01]; Available from: http://epp.eurostat.ec.europa.eu/portal/page/portal/europe_2020_indicators/headline_indicators

26. Wang L-Y, Kick E, Fraser J, Burns TJ. Status attainment in America: The roles of locus of control and selfesteem in educational and occupational outcomes. Sociological Spectrum. 1999;19(3):281-98.

27. Bosma H, Marmot MG, Hemingway H, Nicholson AC, Brunner E, Stansfeld SA. Low job control and risk of coronary heart disease in Whitehall II (prospective cohort) study. BMJ. 1997;314(7080):558.

28. Kohn ML, Schooler C. Job conditions and personality: A longitudinal assessment of their reciprocal effects. American journal of Sociology. 1982:1257-86.

29. Chen E, Miller GE. "Shift-and-Persist" Strategies Why Low Socioeconomic Status Isn't Always Bad for Health. Perspectives on Psychological Science. 2012;7(2):135-58.

30. Bandura A. Self-efficacy: Toward a unifying theory of behavioral change. Psychological Review. 1977;84(2):191-215.

31. Doğan G, van Dijke A, Terpstra L. Wie zijn er moeilijk bereikbaar?: aanknopingspunten voor ondersteuning en begeleiding van'moeilijk bereikbare'gezinnen en jongeren: Nederlands Instituut voor Zorg en Welzijn/NIZW; 2001.

32. Dutch government. Participatiewet [The Participation Act]. overheid.nl; 2016 [cited 2016 03-05]; Available from: http://wetten.overheid.nl/BWBR0015703/2016-04-01.

33. Rossman GB, Wilson BL. Numbers and words combining quantitative and qualitative methods in a single large-scale evaluation study. Evaluation review. 1985;9(5):627-43.

34. Tashakkori A, Teddlie C. Handbook of mixed methods in Social \& Behavioural Research. Thousand oaks: Sage; 2003.

35. Creswell JW, Plano Clark VL, Gutmann ML, Hanson WE. Advanced mixed methods research designs. Handbook of mixed methods in social and behavioral research. 2003:209-40.

36. Greene JC, Caracelli VJ. Advances in mixed-method evaluation: the challenges and benefits of integrating diverse paradigms: Jossey-Bass Publishers; 1997.

37. Fetters MD, Curry LA, Creswell JW. Achieving Integration in Mixed Methods Designs-Principles and Practices. Health Services Research. 2013;48(6pt2):2134-56.

38. The Ministry of Education Culture and Science (VWS), the Ministry of Security and Justice (VenJ), the Association of Netherlands Municipalities. (VNG). Factsheet Nieuw basispakket Jeugdgezondheidszorg [New basicpackage for the child and youth health care]. 2014 [cited 2016 23-4]; Available from: https://vng.nl/onderwerpenindex/jeugd/jeugdgezondheidszorg/publicaties/factsheet-nieuw-basispakket-jgz. 
Summary 



\section{Stay in or drop out}

\section{The pathways to school dropout from a public health perspective}

Dropping out of school is not a discrete event but a dynamic developmental process that begins early in life. It affects individual youngsters, their families and society as a whole. Education level is closely linked to health status and to health disparities. Poor health is involved in pathways toward school dropout and dropping out of school negatively affects health. Furthermore, leaving school without a basic qualification has major socioeconomic consequences. Yet, school dropout is generally considered an educational problem and heavily understudied from a public health perspective. Furthermore, interventions to reduce school dropout are mostly focused on monitoring and enforcing school attendance, respectively deployed late in the dropout process. Few interventions are aimed at tackling the problem at its (potential) roots by identifying children with physical, mental and social problems early in life.

The aim of this thesis is to identify early biopsychosocial determinants in the pathways leading to school dropout from infancy to adolescence. Furthermore, an in-depth understanding into these pathways from the perspectives of the young adults, parents, and educational and health care professionals was aimed at. Such understanding will contribute to the future development of more effective, personalized interventions that will help youth health care (YHC) professionals, teachers, and parents to prevent students from dropping out of school.

In order to reach this aim, this study used a sequential mixed-methods design. Data from the YHC-files and an additional questionnaire were used in a case-control study with 330 young adults (18-23 years) who dropped out of school (case group) or graduated with a delay (controls). The respondents were living in the south-east of the Netherlands. The results of the case-control study were discussed in individual interviews with 25 young adults (cases and controls) to gain an in-depth understanding of the processes that lead to school dropout. The results from the case-control study and the individual interviews were discussed in the subsequent focus groups with parents, educational and health care professionals, respectively.

The following research questions were formulated:

1. What biopsychosocial determinants in infancy, early and middle childhood, and adolescence predict school drop-out in young adulthood (18-23 years)?

2. Are gender beliefs and self-reported masculinity and femininity, beyond the influences of sex, related to school dropout in young adulthood?

3. Are adolescent low control beliefs, independent of parental socioeconomic background, related to school dropout and poor health in young adulthood? 
4. How do young adults who graduated with a delay or dropped out of school perceive their life history and particularly their time in school?

5. How do parents, stakeholders from education and stakeholders from healthcare view the reasons for dropping out of school and their own and each other's roles in preventing school dropout at an early age?

Answers to these research questions are addressed in the chapters 3 to 8 of this thesis.

Chapter 1 provides the introduction to this thesis in terms of school dropout and a public health perspective. It describes how early school leaving or school dropout is defined, and why dropping out of school must be seen as a public health issue. Subsequently, gender differences in school dropout are discussed and the concept of growing into deficit is explained. Furthermore, a description of the Dutch educational and YHC system is given. Finally, the aim and research questions of this thesis are outlined.

Chapter 2 presents the study protocol of the SIODO study. It discusses that where most studies are fragmented and focus on single determinants, the SIODO study uses a multidimensional approach and a life-course perspective to explore many candidate biopsychosocial determinants that might be related to school dropout in young adulthood in one study. Furthermore, it describes that the mixed methods design, where a quantitative case-control study is followed by and input for a qualitative study with interviews and focus groups, will enable us to further interpret and understand the acquired information, to deepen the complex processes to school drop-out and to gain insight into the views of all stakeholders involved. Finally, the strengths and limitation of the SIODO study and the role of the YHC in signalling children at risk of school dropout are discussed.

Chapter 3 addresses the research question "What biopsychosocial determinants in infancy, early and middle childhood, and adolescence predict school drop-out in young adulthood (18-23 years)?" In a case-control design, both life course data from the YHCfiles, collected in the everyday practice of the YHC professionals and an additional questionnaire filled out by 529 youngsters, aged 18-23 years were used. First, univariate logistic regression analyses with school-dropout as the dependent variable were conducted followed by backward and forward stepwise analyses with the significant variables pertaining to the 0-4 year period. Remaining significant variables were forced into the next model and subsequently variables pertaining to, respectively the 4-8, 8-12 and 12-16 year period were introduced in a stepwise analysis. All analyses were crossvalidated in an exploratory and confirmatory random half of the sample. The findings supported the relevance for school dropout in young adulthood (18-23 years) of having a non-Western background, an insufficient hearing test and the birth of a sibling (OR $0.63,95 \% \mathrm{Cl} 0.43-0.93$ ) in the $0-4$ age period, learning difficulties, not attending sports, 
hospitalization or having surgery (protective) in the 4-8 age period, externalizing behaviour in the 8-12 age period (OR 2.81, 95\% Cl 1.53-5.14), and school absenteeism (OR $5.62,95 \% \mathrm{Cl} 2.18-14.52$ ) and self-efficacy (OR 0.53, 95\% $\mathrm{Cl} 0.38-0.74$ ) in the 12-16 age period. In all age periods, a poor relationship between parents increased the odds of dropout and so did an accumulation of stressful life events. Girls with an early puberty are at risk for school dropout and so are girls who got pregnant in their teens. Youngsters with a non-Western background, from one parent-families or living in areas with cheaper houses more often missed one or more health examinations from the $\mathrm{YHC}$, and missing such health examinations was associated with a risk of school dropout. These findings underline that to prevent school dropout, YHC professionals should not wait until imminent dropout, but should identify and tackle risk factors as early as possible and actively approach youngsters who withdraw from public health care.

In chapter 4 the relation between school dropout, sex and gender is described. A casecontrol design (330 dropout cases and 330 controls, aged between 18 and 23 years) was used with self-administered questionnaires. The young adults were asked to rate how they perceived their femininity and masculinity (thinking back to the time they were 16 years old). Furthermore, they were asked to react on (a) 12 belief statements about appropriate behaviour for males into interpersonal relationships among peers (AMIRS questionnaire), and (b) to 10 statements about conventional femininity ideologies of inauthenticity in relationships (based on the ISR scale of the AFIS questionnaire ). Separate logistic regression analyses for the male and female participants, controlled for sociodemographic determinants were conducted. Results showed that school dropout in young adulthood was related to self-rated masculinity/femininity and to gender beliefs, and this relationship differed for female and male young adults. For young women, an intermediate score on the gender variables tended to protect best against dropout. Female participants who dropped out of school were more likely to have lived in one-parent families when in primary school, considered themselves less feminine and scored higher on the AMIRS compared with female participants in the control group. Furthermore, young women who rated themselves high on masculinity were at higher risk of school dropout (OR 2.1,95\% Cl 1.1-4.1). For young men, the gender effect presented itself in terms of interactions. Young men who rated themselves high on masculinity and simultaneously had strong normative beliefs about male peer relationships had a higher risk of dropping out of school and so had young men who considered themselves simultaneously high on femininity and low on masculinity. This study shows that beyond sex, gender is important in the explanation of school dropout. To prevent dropout, public health professionals might assess, monitor and intervene on the basis of gender characteristics.

Chapter 5 focuses on if and how the (indirect) selection mechanism contributes to school dropout and poor health in young adulthood (18-23 years). In a case-control 
design (330 dropout cases and 330 controls, aged between 18 and 23 years), data from the questionnaire were used. Respondents were asked for current health status (on a scale from 1-10), socioeconomic and social background in their childhood, and control beliefs when they were 16 years old. Logistic regression analyses were conducted. Low mastery and low self-efficacy were found to be strongly associated with school dropout and less than good health (a score below 7) in young adulthood. This association was independent of parental socioeconomic background. A lower socioeconomic background, as indicated by measures of recalled relative deprivation and parental education, was also related to school dropout, as was a non-Western background and coming from a one-parent family. For young adults, who had dropped out of school, low socioeconomic background was related to less than good health and estimates of low mastery at the age of 16 . These findings support the indirect selection mechanism. Consequently, since individual differences in control beliefs seemed to be as fundamental as socioeconomic conditions in the pathways toward school dropout, YHC professionals might already start considering early interventions in youth with all too fatalistic and powerless mind-sets.

Chapter 6 describes the findings from the semi-structured interviews with 11 young men and 14 young women, aged 22 to 25 years, who in 2011 were school dropouts (18) or graduated with a delay (7). This qualitative study aimed to gain an in-depth understanding into the processes underneath our previous case-control findings that lead to school dropout from the perspective of the young adults. The topic list was based on the previous case-control findings and the literature. Transcripts were analyzed thematically. The themes that emerged were (1) safety and support, (2) expectations and possibilities, and (3) beyond the individual. Interviewees had to be resilient to finish their education and a sense of control beliefs, self-confidence, and social support were resources. Lacking safety and social support or being (violently) excluded from relevant social groups, because of physical, cognitive and mental limitations, or not conforming to expected group rules decreased their (future) expectations and had negative consequences for their self-esteem and control beliefs. In turn, self-esteem appeared to be important whether someone felt included or not. Focusing on the individual without incorporating the wider context in addressing problems also decreased interviewees' self-esteem. Some young adults, who graduated with a delay, reported feeling accepted and experiencing support from family, school and peers. They felt self-confident and had pleasant memories of their youth and time in school. These findings indicate that public health professionals should play a role in improving students' self-esteem and control beliefs and in including the whole system in case of problems.

In chapter 7, it is explored how parents, educational and healthcare stakeholders view the reasons for dropping out of school and theirs and each other's roles in preventing school dropout at an early age. This will contribute to the future development of inter- 
ventions to prevent school dropout as early as possible, and to the cross-validation of previous findings. Five homogeneous focus groups were conducted, with parents, stakeholders from education and healthcare, respectively. The interview guide was based on previous results from the case-control studies with questionnaires and information from the YHC-files, and the individual interviews with the young adults. A content analysis was performed and four major themes were identified: (1) growing into responsibilities, (2) meeting the norm, (3) building a bridge and (4) bending the rules. All participants recognized the determinants found in previous SIODO studies, and added the importance of having responsibilities. Appropriate for their age and development, children learn how to take responsibility for their own learning process. Expecting too much, may lead to fear of failure and decrease self-confidence. For belonging to a peer group and fitting in the school system, which provides self-confidence and sufficient support, youngsters must meet the norm. Unclear professional roles, medical confidentiality and privacy, and mutual mistrust may hinder stakeholders to cooperate in preventing school dropout. Both the educational and healthcare system are experienced as rigid, performance-driven systems, bound to limiting rules, which had to be bended to provide tailored support. This study concludes that to prevent school dropout, the individual child and his or her environment should be involved. Mutual trust between stakeholders must be restored and the YHC physician may be a linking pin in establishing good cooperation for the sake of the child. Educational and healthcare systems need to be facilitated sufficiently and be more flexible so that professionals can provide tailored support, taking into account individual differences.

Chapter 8 comprises the general discussion of this thesis. First, the mean research findings are integrated and discussed. Topics evaluated include the ICF-CY model and its usefulness for the YHC practice, the developmental background of school dropout and the importance to grow up in a safe environment. Subsequently, the need of a personalized approach is discussed, which contradicts with the necessity to meet the "norm" in today's society. Gender and socioeconomic health differences are also addressed. Methodological strengths and limitations are considered, in particular the mixed methods design with the integration of the quantitative and qualitative methods and findings, providing a broader perspective on the overall issue of school dropout. Finally, recommendations for future research and for practice and policy are offered, including possibilities for a future tool to prevent school dropout from a public health perspective and at an as early as possible age.

This thesis shows that it is possible to signal heightened risks of school dropout from an early age. With a clear registration, and in cooperation with youngsters, parents, school, and other healthcare partners, preventive youth healthcare must be able to contribute to reduce dropout rates and thus socioeconomic health differences. However, dropping out of school is a multidimensional problem, with many complex pathways leading to 
school dropout. It is not about the detection of individual determinants, but the understanding of a coherent whole. Therefore, a questionnaire and checklists are not sufficient. A personalized approach is needed, in which we listen to the story of the youngsters themselves. 
Samenvatting 



\section{Stay in or drop out}

\section{De wegen die leiden tot schooluitval vanuit het perspectief van de publieke gezondheid}

Schooluitval is geen op zichzelf staande gebeurtenis, maar een complex probleem dat al op jonge leeftijd begint. Het heeft invloed op individuele jongeren, hun families en de gehele maatschappij. Opleidingsniveau is nauw verbonden met gezondheid en met gezondheidsverschillen. Een slechte gezondheid kan leiden tot schooluitval en schooluitval beïnvloedt de gezondheid negatief. Bovendien heeft het verlaten van school zonder startkwalificatie grote sociaaleconomische gevolgen. Toch wordt schooluitval meestal beschouwd als een onderwijsprobleem en is er weinig onderzoek gedaan vanuit het perspectief van de publieke gezondheid. Bovendien zijn de meeste interventies om schooluitval te voorkomen gericht op monitoring en handhaving bij afwezigheid op school en zij worden vaak pas laat in het proces ingezet. Weinig interventies zijn gericht op het aanpakken van het probleem bij de (potentiële) oorsprong door kinderen met lichamelijke, psychische en sociale problemen al vroeg in het leven te identificeren.

Het doel van dit proefschrift is het identificeren van bio-psychosociale determinanten die vanaf de vroege levensjaren bijdragen aan voortijdig schoolverlaten. Daarnaast het krijgen van inzicht in de onderliggende processen bij schooluitval vanuit het perspectief van de jong volwassenen, ouders en professionals in het onderwijs en de gezondheidszorg. Hiermee kunnen in de toekomst effectievere en meer gepersonaliseerde interventies worden ontwikkeld, waarmee jeugdgezondheidszorg (JGZ) professionals, docenten en ouders samen helpen voorkomen dat leerlingen op school uitvallen.

Om dit doel te bereiken, is er in dit onderzoek gebruik gemaakt van een sequentieel mixed-methods design. Van 330 jongeren (van 18 tot 23 jaar) uit Zuidoost-Brabant die zonder startkwalificatie de school hebben verlaten (cases) zijn de gegevens uit de jeugdgezondheidszorg-dossiers en een aanvullende vragenlijst vergeleken met die van 330 jongeren van dezelfde leeftijd uit Zuidoost-Brabant die nog wel onderwijs volgden (controles). De resultaten waren input voor individuele interviews met 25 jongeren (cases en controles) om inzicht te krijgen in de onderliggende processen bij schooluitval. Vervolgens zijn de resultaten en implicaties besproken in focus groepen met achtereenvolgens ouders, onderwijs-, en zorgprofessionals.

De volgende onderzoeksvragen zijn geformuleerd:

1. Welke bio-psychosociale determinanten in de verschillende leeftijdsfasen van geboorte tot adolescentie voorspellen schooluitval op jong volwassen leeftijd (18-23 jaar)? 
2. Zijn naast sekse, opvattingen over stereotype genderrollen en zelf gerapporteerde mannelijkheid en vrouwelijkheid gerelateerd aan schooluitval op jong volwassen leeftijd?

3. Is, onafhankelijk van de sociaaleconomische achtergrond van ouders, het hebben van weinig overtuiging in het eigen kunnen in de adolescentie gerelateerd aan schooluitval en een slechte gezondheid op jong volwassen leeftijd?

4. Hoe ervaren jong volwassenen die uitgevallen zijn op school of met een vertraging hun diploma hebben gehaald, hun jeugd en vooral hun schooltijd?

5. Hoe kijken ouders, stakeholders van onderwijs en van gezondheidszorg aan tegen de oorzaken van schooluitval, en tegen hun eigen rol en die van anderen in de preventie van schooluitval op jonge leeftijd?

In de hoofdstukken 3-8 van dit proefschrift wordt antwoord gegeven op deze onderzoeksvragen.

Hoofdstuk 1 leidt dit proefschrift in, wat betreft schooluitval en het perspectief van de publieke gezondheid. De definitie van voortijdig schoolverlaten of schooluitval wordt gegeven en er wordt besproken waarom schooluitval een probleem is van de publieke gezondheid. Vervolgens wordt ingegaan op sekseverschillen in schooluitval en wordt het concept " in een tekort groeien" uitgelegd. Verder wordt er een beschrijving van het Nederlandse onderwijssysteem en de JGZ gegeven. Tot slot worden het doel en de onderzoeksvragen van dit proefschrift beschreven.

Hoofdstuk 2 presenteert het studie protocol van de "Stay in or drop out" (SIODO) studie. Het bespreekt dat waar de meeste studies zijn versnipperd en zich richten op enkele losse factoren de SIODO studie gebruik maakt van een multidimensionale aanpak met een levensloop perspectief om de vele bio-psychosociale factoren die mogelijk kunnen worden gerelateerd aan schooluitval bij jong volwassenen, in één studie te verkennen. Verder wordt het mixed methods design beschreven, waarbij een kwantitatieve casecontrol studie wordt gevolgd door en input is voor een kwalitatief onderzoek met interviews en focusgroepen. Dit stelde ons in staat om de verkregen informatie verder te interpreteren en te begrijpen, om de complexe processen leidend tot schooluitval verder uit te diepen en om inzicht te krijgen in de standpunten van alle betrokken stakeholders. Tot slot worden de sterke punten en de beperking van de SIODO studie besproken en de rol van de JGZ in het signaleren van kinderen die risico lopen om op school uit te gaan vallen.

Hoofdstuk 3 behandelt de onderzoeksvraag: "Welke bio-psychosociale determinanten in de verschillende leeftijdsfasen van geboorte tot adolescentie voorspellen schooluitval op jong volwassen leeftijd (18-23 jaar)?" In een case-control design werd gebruik ge- 
maakt van gegevens uit 529 JGZ-dossiers van jongeren tussen de 18 en 23 jaar, verzameld vanaf de geboorte in de dagelijkse JGZ-praktijk en van een aanvullende vragenlijst. Eerst zijn er univariate logistische regressieanalyses uitgevoerd met schooluitval als afhankelijke variabele. Daarna zijn er backward en forward stepwise regressieanalyses gedaan met de significante variabelen uit de periode van 0-4 jaar. De variabelen die significante bleven, werden in het volgende model "gedwongen" en vervolgens werden variabelen uit respectievelijk de leeftijdsperiodes 4-8 jaar, 8-12 jaar en 12-16 jaar stapsgewijs in de analyse geïntroduceerd. Alle analyses zijn cross-gevalideerd in een verkennende en een bevestigende willekeurige helft van de steekproef. Schooluitval bij jong volwassenen (18-23 jaar) was gerelateerd aan het hebben van een niet-Westerse achtergrond, een onvoldoende gehoortest en de geboorte van een broertje of zusje (OR 0,63, 95\% $\mathrm{Cl} 0,43-0,93$ ) in de periode van 0 - 4 jaar. In de periode van 4-8 jaar met leermoeilijkheden, niet deelnemen aan een sportclub of zwemles, ziekenhuisopname of een operatie (beschermend), en met externaliserend gedrag (OR 2,81, 95\% Cl 1,535,14 ) in de leeftijd van 8-12 jaar. In de leeftijdsperiode 12-16 jaar, was schooluitval gerelateerd aan ziekteverzuim (OR 5,62, 95\% Cl 2,18-14,52) en vertrouwen hebben in de eigen bekwaamheid (OR 0,53, 95\% $\mathrm{Cl} 0,38-0,74)$. In iedere leeftijdsperiode vergrootte een slechte relatie tussen ouders de kans op schooluitval bij hun kinderen, net als een opeenstapeling van stressvolle gebeurtenissen in het leven. Meisjes, die vroeg in de puberteit kwamen en ook meisjes die in hun tienerjaren zwanger raakten, liepen meer risico op schooluitval. Jongeren met een niet-Westerse achtergrond, uit een éénoudergezin of wonend in een gebied met goedkopere huizen hadden vaker één of meerdere JGZ- onderzoeken gemist, en het missen van een JGZ-onderzoek was gerelateerd aan een verhoogd risico op schooluitval. Deze bevindingen onderstrepen het belang voor JGZ professionals die schooluitval willen voorkomen, niet te wachten tot er sprake is van dreigend schooluitval, maar risicofactoren zo vroeg mogelijk te herkennen en aan te pakken. Bovendien moeten zij jongeren die niet verschijnen op een JGZ-onderzoek actief benaderen.

Hoofdstuk 4 beschrijft de relatie tussen schooluitval, sekse en gender. Er is gebruik gemaakt van een case-control design (330 dropouts en 330 controles, tussen de 18 en 23 jaar oud) met vragenlijsten die de jongeren zelf invulden. Aan de jongeren werd gevraagd om een cijfer tussen de 1 en 10 te geven voor hun vrouwelijkheid en mannelijkheid (terugdenkend aan de tijd dat ze 16 jaar oud waren). Daarnaast werd aan hen gevraagd om te reageren op (a) 12 uitspraken over passend gedrag voor mannen in relaties met vrienden (Amirs vragenlijst), en op (b) 10 uitspraken over conventionele vrouwelijke ideologieën over het niet authentiek zijn in relaties (op basis van de ISR schaal van de AFIS vragenlijst). Logistische regressie analyses zijn apart voor de jonge mannen en vrouwen uitgevoerd, gecontroleerd voor sociaal demografische factoren. Schooluitval op 18-23 jarige leeftijd was gerelateerd aan hoe mannelijk en vrouwelijk de respondenten zichzelf vonden en aan hun opvattingen over stereotype genderrollen. 
Deze relaties waren voor mannen en vrouwen verschillend. Voor vrouwen leek een gemiddelde score op alle gender variabelen het beste te beschermen tegen schooluitval. Bij vrouwelijke respondenten die op school uitgevallen waren, was de kans groter dat zij op de basisschool in een eenoudergezin woonden, dat zij zichzelf minder vrouwelijk vonden en dat ze hoger scoorden op de Amirs vragenlijst in vergelijking met vrouwelijke respondenten in de controlegroep. Bovendien hadden de jonge vrouwen die zichzelf een hoog cijfer voor mannelijkheid gaven een hoger risico op schooluitval (OR 2,1, $95 \% \mathrm{Cl} 1,1-4,1)$. Bij mannen was er wat betreft het gender effect op schooluitval sprake van interacties. Jonge mannen die zichzelf hoog scoorden op mannelijkheid en tegelijkertijd sterke normatieve opvattingen over mannelijke relaties met leeftijdsgenoten hadden, hadden een hoger risico op voortijdig schoolverlaten. Dit gold ook voor jonge mannen die zichzelf tegelijkertijd erg vrouwelijk en weinig mannelijk beschouwden. Dit onderzoek toont aan dat behalve sekse, gender een rol speelt bij schooluitval. Om uitval te voorkomen, zouden professionals in de publieke gezondheid aandacht moeten schenken aan gendereigenschappen. Zo nodig moeten problemen met betrekking tot gender gevolgd worden of moet er een interventie plaats vinden.

Hoofdstuk 5 richt zich op de vraag of en hoe het (indirecte) selectiemechanisme bijdraagt aan schooluitval en een slechte gezondheid bij jong volwassenen (18-23 jaar). Er werd een case-control design (330 dropouts en 330 controles, tussen de 18 en 23 jaar oud) met de gegevens uit de vragenlijst gebruikt. De respondenten werd gevraagd een cijfer (op een schaal van 1-10) te geven voor hun huidige gezondheid, hun sociaaleconomische en sociale achtergrond in hun jeugd, en hun overtuiging in het eigen kunnen toen ze 16 jaar oud waren. Er werden logistische regressie analyses uitgevoerd. Een laag gevoel van meesterschap en weinig vertrouwen in het eigen kunnen bleken sterk geassocieerd te zijn met schooluitval en met een minder goede gezondheid (een score lager dan 7) bij jong volwassenen. Deze associatie was onafhankelijk van de sociaaleconomische achtergrond van de ouders. Een lagere sociaaleconomische achtergrond (met als maat de herinnerde relatieve deprivatie en opleiding van de ouders) was ook gerelateerd aan schooluitval, net als het hebben van een niet-Westerse achtergrond en afkomstig zijn uit een eenoudergezin. Een lage sociaaleconomische achtergrond was bij schooluitvallers gerelateerd aan een minder goede gezondheid en het aangeven van een laag gevoel van meesterschap op de leeftijd van 16 jaar. Deze bevindingen ondersteunen het indirecte selectiemechanisme. Individuele verschillen in de overtuiging van eigen bekwaamheid lijken bij de weg naar schooluitval net zo fundamenteel te zijn als sociaaleconomische omstandigheden. JGZ professionals moeten daarom nadenken of zij vroegtijdige interventies in gaan zetten bij jongeren met al te fatalistische en machteloze mind-sets.

Hoofdstuk 6 beschrijft de bevindingen uit de semi gestructureerde interviews met 11 mannen en 14 vrouwen, in de leeftijd 22 tot 25 jaar, die in 2011 schoolverlaters waren 
(18) of afstudeerden met een vertraging (7). Deze kwalitatieve studie had als doel om inzicht te krijgen vanuit het perspectief van de jong volwassenen in hoe de eerdere case-control bevindingen leiden tot schooluitval. De topic list was gebaseerd op de eerdere case-control bevindingen en de literatuur. Transcripten werden thematisch geanalyseerd. De thema's die naar voren kwamen waren (1) veiligheid en ondersteuning, (2) verwachtingen en mogelijkheden en (3) het kind als onderdeel van een systeem. De geïnterviewde jong volwassenen moesten veerkrachtig zijn om hun opleiding af te kunnen maken en benodigde bronnen hiervoor waren vertrouwen in het eigen kunnen, zelfvertrouwen en sociale steun. Gebrek aan veiligheid en sociale steun of (met geweld) worden uitgesloten van relevante sociale groepen, als gevolg van fysieke, cognitieve en mentale beperkingen of omdat je niet voldeed aan de verwachte groepsregels, verminderde (toekomstige) verwachtingen. Dit had negatieve gevolgen voor het gevoel van eigenwaarde en de overtuiging van het eigen kunnen. Omgekeerd, het gevoel van eigenwaarde bleek belangrijk te zijn of iemand werd opgenomen in de groep of niet. Focussen op het individu zonder (de) rest van de omgeving te betrekken in de aanpak van problemen, verminderde ook het gevoel van eigenwaarde van de geïnterviewde jong volwassenen. Sommige participanten, die met een vertraging waren afgestudeerd, meldden dat ze zich wel geaccepteerd voelden en steun van hun familie, school en leeftijdsgenoten hadden ervaren. Ze voelden zich zelfverzekerd en hadden aangename herinneringen aan hun jeugd en de tijd op school. Deze bevindingen geven aan dat public health professionals een rol zouden moeten spelen bij het verbeteren van het gevoel van eigenwaarde van de studenten en het vertrouwen in hun eigen kunnen. In geval van problemen, moet het hele systeem betrokken worden.

In Hoofdstuk 7 is onderzocht hoe ouders, stakeholders in het onderwijs en in de gezondheidszorg aankijken tegen de redenen van schooluitval en tegen hun eigen en elkaars rol in het voorkomen van schooluitval vanaf jonge leeftijd. De bevindingen dragen bij aan de toekomstige ontwikkeling van interventies om schooluitval zo vroeg mogelijk te voorkomen, en de cross-validatie van eerdere bevindingen. Vijf homogene focusgroepen werden uitgevoerd, met respectievelijk ouders, stakeholders uit het onderwijs en de gezondheidszorg. De interview guide was gebaseerd op eerdere resultaten uit de case-control studies met vragenlijsten en informatie uit de JGZ-dossiers en op de bevindingen uit de individuele interviews met de jong volwassenen. Een content analyse werd uitgevoerd en er werden vier grote thema's geïdentificeerd; (1) groeien in verantwoordelijkheden, (2) voldoen aan de norm, (3) bouwen van een brug en (4) buigen van de regels. Alle deelnemers herkenden de determinanten uit de eerdere SIODO studies, en voegden het belang van het hebben van verantwoordelijkheden toe. Conform leeftijd en ontwikkeling leren kinderen verantwoordelijkheid te nemen voor hun eigen leerproces. Het hebben van te hoge verwachtingen kan leiden tot faalangst en het zelfvertrouwen doen verminderen. Om tot een peer groep te horen en in het schoolsysteem te passen, wat zelfvertrouwen en voldoende steun geeft, moeten jongeren vol- 
doen aan de norm (gemiddeld zijn). Onduidelijke professionele rollen, het medische beroepsgeheim en privacy, en onderling wantrouwen kunnen stakeholders belemmeren om samen te werken bij het voorkomen van schooluitval. Zowel het onderwijs als de gezondheidszorg worden ervaren als rigide, prestatiegerichte systemen, gebonden aan beperkende regels, die gebogen moesten worden om ondersteuning op maat te kunnen bieden. Deze studie concludeert dat om schooluitval te voorkomen, zowel het individuele kind als zijn/haar omgeving moet worden betrokken. Wederzijds vertrouwen tussen de stakeholders moet worden hersteld en de JGZ-arts zou een verbindende factor kunnen zijn in het tot stand brengen van een goede samenwerking in het belang van het kind. Onderwijs en gezondheidszorgsystemen moeten voldoende worden gefaciliteerd en moeten flexibeler zijn, zodat professionals ondersteuning op maat kunnen bieden, rekening houdend met individuele verschillen.

Hoofdstuk 8 omvat de algemene discussie van dit proefschrift. Eerst worden de belangrijkste kwantitatieve en kwalitatieve onderzoeksresultaten geïntegreerd en besproken.

Onderwerpen die worden geëvalueerd zijn onder meer het ICF-CY-model en de bruikbaarheid hiervan voor de praktijk van de JGZ, het proces van schooluitval in de ontwikkeling en het belang om op te groeien in een veilige omgeving. Vervolgens wordt de noodzaak van een gepersonaliseerde aanpak besproken, die in tegenspraak is met de noodzaak om aan " de norm" in de huidige maatschappij te voldoen. Daarna wordt ingegaan op het meten van het concept gender en op sociaaleconomische gezondheidsverschillen. Methodologisch sterkte punten en beperkingen van de SIODO studie worden beschouwd, in het bijzonder het mixed methods design met de integratie van de kwantitatieve en kwalitatieve methoden en bevindingen, waardoor een bredere kijk op het schooluitval probleem mogelijk was. Ten slotte zijn er aanbevelingen voor toekomstig onderzoek en voor de praktijk en het beleid, met inbegrip van mogelijkheden voor een toekomstig instrument om schooluitval op een zo vroeg mogelijke leeftijd te voorkomen vanuit het perspectief van de publieke gezondheid.

Dit proefschrift laat zien dat het mogelijk is om een verhoogd risico op schooluitval al op jonge leeftijd te signaleren. Met een duidelijke registratie, en in samenwerking met jongeren, ouders, school en andere partners in de zorg, moet de JGZ in staat zijn om bij te dragen aan het verminderen van schooluitval en dus van sociaaleconomische gezondheidsverschillen. Echter, voortijdig schoolverlaten is een multidimensionaal probleem, en er zijn veel complexe wegen die tot schooluitval kunnen leiden. Het gaat dan ook niet om het opsporen van individuele determinanten, maar het begrijpen van een samenhangend geheel. Daarom zijn een vragenlijst en een checklist niet voldoende. Er is een gepersonaliseerde aanpak nodig waarbij geluisterd wordt naar het verhaal van de jongeren zelf. 


\section{Valorisatie}



Valorisatie kan gedefinieerd worden als het geschikt en beschikbaar maken van wetenschappelijke kennis voor de praktijk. De onderzoeksvragen in dit proefschrift zijn geïnspireerd door de dagelijkse praktijk van de jeugdgezondheidszorg (JGZ) en er is gebruik gemaakt van gegevens die in de JGZ praktijk zijn verzameld. De resultaten uit dit proefschrift zijn daarom niet alleen wetenschappelijk relevant maar lenen zich ook goed voor valorisatie.

\section{Een maatschappelijk probleem}

Voortijdig schoolverlaten is een groot maatschappelijk probleem (1). Jongeren die zonder startkwalificatie de school verlaten vinden moeilijker een (goed betaalde) baan, doen vaker een beroep op een sociale uitkering en bevinden zich vaker in een lagere sociaaleconomische positie dan jongeren met een startkwalificatie (2). Uit diverse studies blijkt dat voortijdig schoolverlaters niet alleen op school uitvallen maar ook in de maatschappij, waarbij ze vaker in de criminaliteit belanden $(3,4)$. Schooluitval brengt daarom hogere kosten voor de maatschappij met zich mee, ook omdat er minder inkomsten zijn uit belasting en er vaker een beroep gedaan wordt op sociale uitkeringen.

Opleidingsniveau en gezondheid zijn nauw met elkaar verbonden (5). Een slechte gezondheid kan leiden tot schooluitval en schooluitval beïnvloedt de gezondheid negatief. Mensen met een lage opleiding en met een lage sociaaleconomische positie zijn meestal minder gezond en leven minder lang. Hoe hoger het opleidingsniveau, hoe beter de gezondheid $(6,7)$. Preventie van schooluitval draagt op deze manier dus bij aan het verminderen van sociaaleconomische gezondheidsverschillen.

\section{Relevantie voor de praktijk}

Dit proefschrift levert bouwstenen voor het ontwikkelen van een (SIODO) tool om te voorkomen dat kinderen op school uit gaan vallen. Tot nu toe is vooral onderzoek naar schooluitval gedaan vanuit een onderwijskundig perspectief. De meeste interventies zijn gericht op het onderwijs en worden pas laat ingezet, vaak bij (dreigende) uitval $(8,9)$. In dit proefschrift is schooluitval benaderd vanuit het oogpunt van de JGZ en de resultaten kunnen bijdragen aan de ontwikkeling van een signaleringsinstrument en interventies die gericht zijn op de jonge levensjaren. De bevindingen in dit proefschrift zijn in dit opzicht innovatief.

De onderzoeksresultaten zijn niet alleen relevant voor wetenschappers, maar vooral ook voor professionals in de JGZ en in het onderwijs, hun managers en beleidsmakers. Bovendien zullen bij het toepassen van deze kennis, kinderen/jongeren en hun ouders profiteren. Door risico's in een vroeg stadium te signaleren en competenties die nodig zijn om met deze uitdagingen om te gaan al op jonge leeftijd te versterken, is naast 
schooluitval ook veel persoonlijk leed te voorkomen. De JGZ is onafhankelijk, heeft contact met school en jongeren, kan medische zorg en onderwijs verbinden en zorgt voor continuïteit van zorg. Dit biedt mogelijkheden om samen met het onderwijs schooluitval vroegtijdig te signaleren en tot een passend aanbod van onderwijs en zorg te komen om zo de kans op uitval te verkleinen. Met het te ontwikkelen signaleringsinstrument kunnen vroege signalen makkelijker door de JGZ en het onderwijs worden herkend en kan een handreiking worden gegeven hoe zij hiermee om kunnen gaan. Managers en beleidsmakers kunnen met het faciliteren van zorg en onderwijs op maat een bijdrage leveren aan het terugdringen van schooluitval en gevolgen als sociaal economische gezondheidsverschillen. In hoofdstuk 8 van dit proefschrift worden aanbevelingen gegeven voor praktijk en beleid

\section{Kennisverspreiding}

De resultaten uit deze studie zijn met verschillende belanghebbenden gedeeld en zijn op diverse manieren in de praktijk verspreid. Zo zijn de resultaten uit de case control studies besproken met de artsen van de GGD Brabant zuidoost en is er gediscussieerd over de bruikbaarheid van de bevindingen in de JGZ praktijk. Met ouders, professionals uit het onderwijs en de zorg zijn de onderzoeksresultaten besproken in 5 verschillende focusgroepen, waarin aan de hand van casuïstiek, gebaseerd op de resultaten tot dan toe, mogelijkheden voor interventies zijn verkend. Er zijn diverse presentaties van de onderzoeksgegevens gehouden op congressen (EUPHA, NCVGZ), refereerbijeenkomsten (in Maastricht en Tilburg) en in kleiner verband bij het thuiszittersoverleg van de regio Helmond Peelland, bij een bijeenkomst met JGZ artsen en leerplichtambtenaren in Eindhoven en bij het kennisnetwerk 'aanval op schooluitval'.

Een aantal bevindingen kunnen direct in de praktijk worden toegepast, zoals het stellen van de vraag of een kind/jongere het gevoel heeft dat hij/zij er bij hoort en het bespreken van diversiteit en acceptatie in de klas. Maar om schooluitval in een vroeg stadium adequaat en structureel aan te pakken zijn een goede signalering en effectieve gepersonaliseerde interventies nodig. De bevindingen uit dit proefschrift kunnen hiervoor worden gebruikt.

\section{De SIODO tool}

In hoofdstuk 8 van dit proefschrift wordt beschreven dat de ICF-CY in aangepaste vorm een geschikt signaleringsinstrument kan zijn om in de JGZ risico's op te sporen en de ontwikkeling van kinderen te volgen. Hieronder wordt ingegaan op mogelijkheden voor de ontwikkeling van effectieve gepersonaliseerde interventies, die in een vroeg stadium 
door de JGZ kunnen worden ingezet om te voorkomen dat kinderen uit gaan vallen. De preventieve interventies kunnen op verschillende niveaus worden ingezet.

1. Collectieve preventie programma's voor alle kinderen, rekening houdend met de leeftijd, het taalbegrip en cultuur. Deze programma's moeten onder andere de mentale veerkracht en acceptatie van diversiteit vergroten en kunnen zowel op de peuterspeelzaal, de basisschool als op het voortgezet onderwijs aangeboden worden. Bij de ontwikkeling en uitvoering van deze programma's is het belangrijk dat leerkrachten en ouders worden betrokken.

2. Selectieve preventie strategieën op het niveau van multi-screening gericht op het voorkomen van schooluitval, door signalering van negatieve ervaringen uit de jeugd en bepaalde risicofactoren tijdens de levensloop. Op dit niveau kan anticiperende begeleiding worden geboden, zodat tijdig wordt geïntervenieerd bij veel voorkomende gedrags-, emotionele en sociale problemen in de verschillende leeftijdsfasen. Afhankelijk van de uitkomst van de multi-screening kan een beoordeling aan de hand van een gesprek plaatsvinden. In de JGZ wordt al gebruik gemaakt van multi-screening programma's, door deze aan te passen kan ook een verhoogd risico op schooluitval worden opgespoord.

3. Geïndiceerde preventie. Hierbij valt te denken aan het tijdig verwijzen en/of begeleiden van kinderen en jongeren met lichamelijke of mentale beperkingen of met symptomen die het functioneren op school bemoeilijken zoals leerproblemen, slechte schoolprestaties of schoolverzuim.

In het geval van (dreigende) schooluitval, is een interventie op maat nodig om kinderen weer naar school te laten gaan. Zo'n gepersonaliseerde interventie begint met het onderzoeken van risico- en beschermende factoren in de verschillende domeinen van de ICF-CY. Hiermee kan worden bepaald welke volgende stappen nodig zijn voor en afgestemd op de betreffende jongere. Samen met de JGZ-professionals, kunnen jongeren en hun ouders dan beslissen wat behulpzaam zal zijn en worden er duidelijke doelen gesteld.

De SIODO tool zal na ontwikkeling op grote schaal moeten worden geëvalueerd. Het opnemen van de SIODO tool in bijvoorbeeld de GGD Academy, de opleiding tot jeugdarts en jeugdverpleegkundige, de PABO en de lerarenopleiding draagt bij aan een structurele inbedding in de praktijk van zowel de JGZ als het onderwijs. De ontwikkeling en evaluatie van de SIODO tool is afhankelijk van externe financiering. 


\section{Referenties}

1. Freudenberg N, Ruglis J. Reframing school dropout as a public health issue. Prev Chronic Dis. 2007 Oct;4(4):1-11.

2. De Witte K, Cabus S, Groot W, Maassen van den Brink H. Voortijdig schoolverlaten. Rapportage van TIER/platform31: TIER-Maastricht university2014.

3. OECD. The Organisation for Economic Co-operation and Development. 2008; Available from: www.oecd.org.

4. Lochner L, Moretti E. The Effect of Education on Crime: Evidence from Prison Inmates, Arrests, and SelfReports. American Economic Review. 2004;94(1):155-89.

5. Breslau J. Health in Childhood and adolescence and High School Dropout: University of California, Davis2010.

6. Molla M, Madans J, Wagener D. Differentials in adult mortality and activity limitation by years of education in the United States at the end of the 1990s. Population and Development Review. 2004;30:625-46.

7. Mackenbach JP. Health inequalities: Europe in profile. London: COI.2006.

8. De Witte K, Cabus SJ. Dropout prevention measures in the Netherlands, an evaluation TIER-Maastricht University2010.

9. Tanner-Smith E, Wilson S. A Meta-analysis of the Effects of Dropout Prevention Programs on School Absenteeism. Prevention Science. 2013 2013/10/01;14(5):468-78. 
Dankwoord 
Nooit te oud!

Elke dag mag ik leren

Van alles en iedereen.

Van alles wat gezegd

En gedaan wordt om me heen.

Voor allemaal nu een bedankje

ledereen doet wel iets.

Echt! Zonder jullie allen

Was ik helemaal niets!

Ben van Ooijen 


\section{Dankwoord}

Op mijn weg naar dit proefschrift heb ik ontzettend veel geleerd, me vaak verwonderd en vooral genoten. Ik heb deze weg niet alleen afgelegd, een groot aantal mensen heeft meegedacht, meegeholpen en/of meegeleefd. Een woord van dank is dan ook op zijn plaats.

Allereerst wil ik alle jongeren die de vragenlijst hebben ingevuld bedanken en in het bijzonder de jongens en meisjes die mij zo openhartig hun levensverhaal hebben toevertrouwd. Ik heb heel veel van jullie geleerd. Ook de ouders en professionals in het onderwijs en in de zorg die deelgenomen hebben aan de focusgroepen wil ik heel hartelijk bedanken. Zonder jullie medewerking was dit onderzoek nooit mogelijk geweest.

Beste Frans, jouw deskundigheid, enthousiasme en humor inspireerden me elke keer opnieuw. Naast wetenschapper ben je ook jeugdarts in hart en nieren en dat maakt je aandeel in dit proefschrift zo waardevol. Ik heb veel bewondering voor de manier waarop je mij hebt begeleid. Ik hoop van harte dat we onze samenwerking kunnen continueren en de bevindingen uit dit proefschrift in de praktijk kunnen gaan toetsen. Beste Hans, van jou heb ik veel geleerd over kwantitatief onderzoek en het analyseren met behulp van SPSS. Je stimuleerde me om zo kort en krachtig mogelijk te schrijven en hoe lastig dat vaak ook was, het artikel werd er altijd beter van. Je snelle reactie, kritische blik en focus op sociaal economische gezondheidsverschillen waren heel waardevol voor mijn leerproces en voor dit proefschrift. Beste Petra, jij hebt mij de waarde en kunst van het kwalitatief onderzoek bijgebracht. De analyse van de interviews vond ik erg lastig omdat alles met alles samen hangt, maar met jouw begeleiding lukte het om de thema's te herkennen. Met veel plezier denk ik ook terug aan het congres in Glasgow en jouw aanwezigheid. Petra, jouw deskundigheid ook op het gebied van diversiteit en gender, je betrokkenheid en hartelijkheid hebben absoluut een positieve bijdrage geleverd aan dit proefschrift. Ik had me geen beter promotieteam kunnen wensen. Frans, Hans en Petra, heel hartelijk bedankt voor al jullie tijd en inspanningen en voor het vertrouwen dat jullie mij gegeven hebben. Ons SIODO overleg was altijd inspirerend, er werd veel besproken, maar ook veel gelachen. We vulden elkaar goed aan, maar er was ook ruimte voor verschillen. Ik zal deze bijeenkomsten missen!

De leden van de leescommissie; Prof.dr.T.A.M.J. van Amelsvoort, Prof.dr.M.R.J. Crul, Prof dr. K. Horstman, Prof.dr.ir. M.W.J. Jansen en Prof.dr. S.A. Reijneveld wil ik bedanken voor de tijd en energie die zij besteed hebben aan het beoordelen van mijn manuscript.

Ilse, Inge, Lobke en Madelien, jullie hebben met je wetenschappelijke stage een belangrijke bijdrage geleverd aan de dataverzameling, de analyse en het meeschrijven aan 
artikelen en dus aan dit proefschrift. Bovendien heb ik een hele gezellige tijd met ieder van jullie gehad en Ik wil jullie hier dan ook heel hartelijk voor bedanken.

Ook Sophie en Robin die de gegevens uit de JGZ dossiers hebben ingevoerd, en Hellen die de interviews heeft uitgewerkt wil ik bedanken. Jullie hebben me veel werk uit handen genomen.

De afdeling leerplicht van de gemeente Eindhoven bedank ik voor het verstrekken van de gegevens van de doelgroep.

Het viel niet altijd mee om in het Engels te schrijven en te spreken. Cil, bedankt dat jij het interviewartikel hebt gelezen en het Engels hebt gecorrigeerd waar nodig. Peter v.d. L., dankzij jouw hulp had ik meer zelfvertrouwen tijdens mijn presentatie in Glasgow, bedankt hiervoor!

Peter v. N., wij zijn al meer dan 20 jaar GGD collega's. Je hebt me tijdens mijn specialisatie tot arts maatschappij en gezondheid geholpen bij het onderzoek naar XTC gebruik bij middelbare scholieren en daar is ook mijn interesse in wetenschappelijk onderzoek ontstaan. Daarnaast ben je een goede vriend. Het was voor mij dan ook heel vanzelfsprekend dat jij paranimf zou worden. Marjo, wij kennen elkaar minder lang maar zeker niet minder goed. Tijdens de ruim $900 \mathrm{~km}$ die we samen gelopen hebben, hebben we lief en leed gedeeld. Jij hebt het grootste deel van mijn promotietraject meegemaakt en meegeleefd en ik voelde me altijd begrepen en gesteund. Ik ben blij dat je mijn paranimf wilt zijn.

Annelie Stevens, directeur van de GGD Brabant Zuidoost en Marjo Blom, sectormanager JGZ, bedankt voor de mogelijkheid die jullie mij hebben geboden om dit promotieonderzoek uit te voeren.

Mijn JGZ collega's van de GGD wil ik bedanken voor hun interesse, geduld en begrip dat ik minder voor het uitvoerend werk van JGZ beschikbaar was. Ook de medewerking bij de focusgroepen en de hulp bij het werven van professionals uit de zorg en het onderwijs waardeer ik enorm. Bij het opmaken, versturen, inscannen en digitaal verwerken van de vragenlijsten en voor het opvragen van de JGZ dossiers heb ik hulp gekregen van het secretariaat en de administratie JGZ, collega's van de postverwerking en van de afdeling gezondheidsbevordering. Ook jullie wil ik heel hartelijk bedanken.

Ik bedank ook mijn collega's bij Tranzo en in het bijzonder mijn (ex) kamergenootjes Wil, Meriam, Antje en Wendy voor hun belangstelling en gezelligheid. Het is fijn om ervaringen uit te wisselen en jullie mening te horen. Meriam, ik vind het ook super fijn dat jij mijn focusgroep artikel van feedback hebt voorzien! Yvonne, jou wil ik speciaal bedan- 
ken voor je bemoedigende woorden en nuttige tips bij de voorbereiding van de verdediging, ik heb mazzel met jou.

Mijn collega's bij de vakgroep sociale geneeskunde geven mij altijd het gevoel dat ik er bij hoor, ook al ben ik er maar weinig. Daarvoor en voor jullie interesse wil ik jullie heel hartelijk bedanken. Isel, jou wil ik speciaal bedanken voor je hulp met alle papieren o.a. rondom de promotie en natuurlijk voor het welkome gevoel dat je me altijd geeft!

Tijdens de masterclass in Rolduc kreeg ik de smaak om onderzoek te doen echt te pakken. Mijn medestudenten en de enthousiaste begeleiding van Maria hebben hier zeker een rol bij gespeeld, bedankt hiervoor!

Liandel, ik prijs me gelukkig met een zus zoals jij. Met jou is het altijd gezellig en je geeft me steeds opnieuw inspiratie en energie. Bedankt ook voor het meelezen van de Nederlandstalige hoofdstukken. Ik verheug me er op om jouw boek gedrukt te zien.

Ik kijk terug op een fijne jeugd, een warm nest waarin ik alle mogelijkheden kreeg om te ontwikkelen en te studeren. Lieve mama, je hebt altijd in mij geloofd, me gesteund en me geholpen waar je kon. Ik ben trots op een moeder zoals jij! Mijn vader en schoonouders mogen er helaas niet meer bij zijn. Stiena, het betekent veel voor me dat je zelfs tijdens je ziekte naar mijn onderzoek vroeg, terwijl je het zelf al zo zwaar had. Papa, Harrie en Stiena, ik weet zeker dat jullie ook trots op me zouden zijn.

ledereen die niet genoemd is, maar die direct of indirect meegeholpen heeft aan het tot stand komen van dit proefschrift dank ik heel hartelijk voor hun steun.

Dit proefschrift was er nooit gekomen als het thuisfront niet achter me had gestaan. Niet alleen toonden jullie begrip dat ik zoveel tijd op mijn kamertje doorbracht, jullie hebben ook geholpen met hand en spandiensten zoals het (laten) testen van de vragenlijsten en het versturen daarvan. Dit "boekje" had er ook nooit zo mooi uitgezien zonder jouw creativiteit Karlijn! Lieve Bas, Jasper en Karlijn, jullie aanwezigheid, gezelligheid, humor en knuffels hebben absoluut positief bijgedragen aan het hele promotietraject. Jullie zijn voor mij het allerbelangrijkst! Lieve Chiel, het is altijd fijn om met jou over mijn onderzoek te praten. Jouw gezelligheid, luisterend oor en oprechte belangstelling waardeer ik enorm.

Lieve Hans, dit traject was voor jou zwaarder dan voor mij. Je hebt me heel wat uurtjes moeten missen. Je was er al die tijd voor mij en zorgde dat ik me met mijn onderzoek bezig kon houden. Je hebt me letterlijk en figuurlijk alle ruimte gegeven om de weg naar dit proefschrift te bewandelen. Ik verheug me erop om samen weer meer tijd door te brengen. 



\section{Curriculum Vitae}

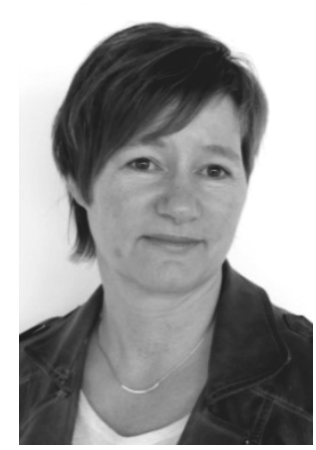

Marie-José Theunissen-Lamers is geboren op 25 februari 1962 te Uithoorn. Ze heeft haar jeugd doorgebracht in Nijmegen en haalde in 1980 haar VWO-B diploma aan het 'Stedelijk Scholengemeenschap Nijmegen'. Marie-José studeerde geneeskunde aan de Katholieke Universiteit Nijmegen waar zij in 1990 haar artsexamen behaalde. Na haar artsexamen is zij gaan werken in de jeugdgezondheidszorg (JGZ) 4-18 jaar en heeft daarnaast een jaar als consultatiebureau arts voor kinderen van 0-4 jaar gewerkt en als arts-assistent op de afdeling dermatologie van het toenmalige St Joseph ziekenhuis in Veldhoven. In Leiden volgde ze de postacademische opleiding sociale geneeskunde bij het NIPG-TNO en in januari 1997 verkreeg ze daar haar registratie als arts maatschappij en gezondheid, profiel jeugdgezondheidszorg. In januari 2001 is Marie-José begonnen met een management development traject, uitgaande van een systeemtheoretische benadering. Tijdens dit traject is zij de doktersassistenten gaan coachen en heeft zij het volledige vaccinatiebeleid onder haar hoede gekregen. In november 2002 heeft zij dit traject met een certificaat afgerond en is haar takenpakket verrijkt met het begeleiden van de artsen en verpleegkundigen bij de invoering en implementatie van standaarden en protocollen. In 2003 werd zij aangesteld als medisch eindverantwoordelijke JGZ en naast het uitvoeren van staftaken was zij verantwoordelijk voor het begeleiden van coassistenten en artsen in opleiding tot sociaal geneeskundige. Van 2005 tot 2008 was zij werkzaam als afdelingshoofd van de afdeling JGZ van de GGD Eindhoven. Naast de afdeling, heeft zij diverse projecten geleid, onder andere het project 'ziekteverzuimbegeleiding bij middelbare scholieren' en 'preventieve aanpak meisjesbesnijdenis', waarbij zij ook de rol van voorzitter van het landelijk projectleidersoverleg had. In deze periode heeft zij ook de module 'innoveren en implementeren van vernieuwingen' uit de opleiding master of Public Health van de NSPOH in Amsterdam gevolgd, en in 2007 met een certificaat afgesloten. In januari 2008 keerde zij na de fusie tussen GGD Eindhoven en GGD Zuidoost Brabant terug in de uitvoering en kreeg de kans om deel te nemen aan de masterclass van de Academische werkplaats publieke gezondheid Limburg, gericht op wetenschappelijke vorming van praktijkprofessionals. Tijdens de masterclass is haar belangstelling voor het wetenschappelijk onderzoek verder gegroeid. In 2009 rondde Marie-José de master- 
class af met een artikel over haar onderzoek 'Are mental health and binge drinking associated in Dutch adolescents?' en ontving hiervoor de 'Cor Spreeuwenberg-prijs', een prijs voor de beste deelnemer van de masterclass, die bruggen weet te slaan tussen 'Beleid, Onderzoek en Praktijk' in de publieke gezondheidszorg. Als science practitioner werkt zij sinds september 2009 bij de Academische werkplaats publieke gezondheid van Tranzo in Tilburg, waar zij een verbinding maakt tussen praktijk en wetenschap. Op het gebied van onderwijs heeft zij diverse activiteiten uitgevoerd, van het opzetten en uitvoeren van een cursus 'opvang van ongevallen op de basisschool' voor leerkrachten en ondersteunend onderwijspersoneel, tot het lesgeven aan praktijkassistenten bij het NIPA in Eindhoven. Van 1995 tot 2011 was zij als arts docent EHBO en reanimatie aan de EHBO vereniging Aalst-Waalre verbonden. Tijdens haar promotieonderzoek 'Stay in or drop out. The pathways to school dropout from a public heath perspective', heeft zij zes master studenten begeleid. Marie-José is getrouwd met Hans Theunissen en samen hebben zij drie kinderen: Bas (1992), Jasper (1992) en Karlijn (1995). 


\section{Publications}

\section{Present thesis:}

\section{Chapter 2:}

Theunissen M-J, Griensven van I, Verdonk P, Feron F, Bosma H. The early identification of risk factors on the pathway to school dropout in the SIODO study: a sequential mixed-methods study. BMC Public Health. 2012;12(1):1033.

\section{Chapter 3:}

Theunissen M-J, Bosma H, Verdonk P, Feron F. Why Wait? Early Determinants of School Dropout in Preventive Pediatric Primary Care. PLoS One. 2015;10(11):e0142315.

\section{Chapter 4:}

Theunissen M-J, de Man I, Verdonk P, Bosma H, Feron F. Are Barbie and Ken too cool for school? A case-control study on the relation between gender and dropout. The European Journal of Public Health. 2015;25(1):57-62.

\section{Chapter 5:}

Bosma H, Theunissen M-J, Verdonk P, Feron F. Low control beliefs in relation to school dropout and poor health: findings from the SIODO case-control study. BMC Public Health. 2014;14(1):1237.

\section{Chapter 6:}

Theunissen M, Verdonk P, Bosma H, Feron F. "I think that the whole family needed help, but they only focused on me" An interview study with young adults about their childhood and dropping out of school (submitted for publication).

\section{Chapter 7:}

Theunissen M, van Maren M, Bosma H, Feron F, Verdonk P. "Don't you have faith in them?" A focus group study with stakeholders in the process toward school dropout (submitted for publication). 


\section{Publications outside this thesis}

Bosma H, Theunissen MJ, Verdonk P, Feron F. School dropout and poor health: causation, direct selection, or indirect selection. International Journal of Behavioral Medicine, 2014: 21 (Supplement 1); S154 (abstract).

Theunissen M, de Man I, Verdonk P, Bosma H, Feron F. Are Barbie and Ken too cool for school? A case-control study on the relation between gender and dropout. European Journal of Public Health, Vol. 24, Supplement 2, 2014.

\section{General publications}

Lamers J.A.M., Driessen W.M.M. Het cholangiocarcinoom bij colitis ulcerosa. Medisch Journaal 1989;18(2):127-129.

Theunissen-Lamers JAM. XTC-gebruik bij middelbare scholieren in Eindhoven. Tijdschrift voor jeugdgezondheidszorg 1999;31:26-27.

Theunissen M-J, Jansen M, van Gestel A. Are mental health and binge drinking associated in Dutch adolescents? Cross-sectional public health study. BMC Research Notes. 2011;4(1):100.

\section{Presentations}

Onderzoeksopzet SIODO en bevindingen van de literatuurstudie. Presentatie "bijeenkomst jeugdartsen en leerplichtambtenaren", Eindhoven, Nederland (november 2010).

Onderzoeksopzet SIODO. Presentatie " bijeenkomst SIODO projectgroep", Eindhoven, Nederland (januari 2011).

The SIODO study design. Posterpresentatie "CAPHRI research day", Maastricht, Nederland (april 2011).

Stay in or drop out, study design. Presentatie "Seminar Social Medicine", Maastricht, Nederland (november 2011).

Ziekteverzuim en schooluitval. Presentatie “Overeindse dagen”, Venetië, Italië (april 2013). 
Stay in or drop out, bevindingen uit de case-control studie. Presentatie "Bijeenkomst kinderartsen sociale pediatrie Zuid Nederland", Helmond, Nederland (september 2013).

Stay in or drop out; de relatie tussen sekse, gender en schooluitval. Presentatie "PAOG jeugdartsen en verpleegkundig specialisten", Maastricht, Nederland (oktober 2013).

Stay in or drop out; onderzoeksopzet en bevindingen uit de case control studies. Presentatie "bijscholing artsen GGD Brabant Zuidoost", Eindhoven, Nederland (mei 2014).

Interpreting early symptoms on the pathway to school dropout: challenges for preventive child and youth healthcare. Presentatie "refereerbijeenkomst Tranzo", Tilburg, Nederland (juni 2014).

School dropout and poor health: causation, direct selection, or indirect selection. Presentatie "ICBM", Groningen, Nederland (augustus 2014).

Are Barbie and Ken too cool for school? Presentatie "EUPHA", Glasgow, UK (november 2014).

The early identification of risk factors on the pathway to school dropout. Presentatie "refereerbijeenkomst Social Medicine", Maastricht. (december 2014).

Resultaten uit de SIODO studie. Presentatie "Stuurgroep onderwijs Helmond-Peelland", Helmond, Nederland (september 2015).

Vroegtijdig signaleren van determinanten die leiden tot voortijdig schoolverlaten. Presentatie "NCVGZ", Rotterdam, Nederland (april 2016).

Het hoofd boven water houden; over veiligheid en schooluitval. Presentatie "Refereeravond Academische werkplaats Zuid-Limburg" Maastricht, Nederland (april 2016).

Resultaten uit de SIODO studie. Presentatie "Kenniskring VSV Zuidoost Brabant", Eindhoven, Nederland (mei 2016). 
